Louisiana State University

LSU Digital Commons

2013

\title{
A Large Scale Network Model to Obtain Interwell Formation Characteristics
}

SeyedmohammadAmin Gherabati

Louisiana State University and Agricultural and Mechanical College

Follow this and additional works at: https://digitalcommons.Isu.edu/gradschool_dissertations

Part of the Petroleum Engineering Commons

\section{Recommended Citation}

Gherabati, SeyedmohammadAmin, "A Large Scale Network Model to Obtain Interwell Formation Characteristics" (2013). LSU Doctoral Dissertations. 362.

https://digitalcommons.Isu.edu/gradschool_dissertations/362

This Dissertation is brought to you for free and open access by the Graduate School at LSU Digital Commons. It has been accepted for inclusion in LSU Doctoral Dissertations by an authorized graduate school editor of LSU Digital Commons. For more information, please contactgradetd@lsu.edu. 


\title{
A LARGE SCALE NETWORK MODEL TO OBTAIN INTERWELL FORMATION CHARACTERISTICS
}

\author{
A Dissertation \\ Submitted to the Graduate Faculty of the \\ Louisiana State University and \\ Agricultural and Mechanical College \\ in partial fulfillment of the \\ requirements for the degree of \\ Doctor of Philosophy
}

in

The Craft \& Hawkins Department of Petroleum Engineering

by

SeyedMohammadAmin Gherabati

B.S., Petroleum University of Technology, 2005

M.S., Amirkabir University of Technology, 2007

December 2013 


\section{Acknowledgments}

I would like to thank my advisor, Dr Richard Hughes, for the support and guidance he showed me through out my research and freedom he gave me in choosing research direction. I am sure it would have not possible been possible without his help.

I am truly indebted and thankful to my committee members; Dr. Christopher D. White who offered stimulating suggestions, Dr. Hongchao Zhang who provided me with his derivative free optimization code, and Dr. Mayank Tyagi and Dr. Karsten Thompson for their thoughtful insights.

I owe sincere and earnest thankfulness to Mr. Shah Kabir and Dr. Matt Honarpour of Hess Corporation for their interest and invaluable comments on the research.

Finally, I would like to thank my family, Nasser, Sedigheh, Zahra, and Sara. Thanks for supporting me during my studies and urging me on. Maman and Baba, you are wonderful parents and wonderful friends. Zahra and Sara, I could not ask for better sisters. To all of you, thanks for always being there for me.

This dissertation is dedicated to my wonderful parents, Nasser and Sedigheh. 


\section{Contents}

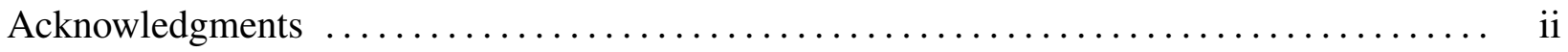

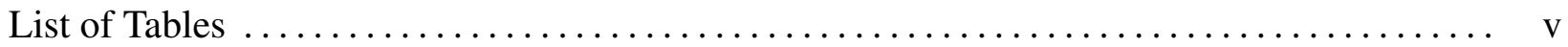

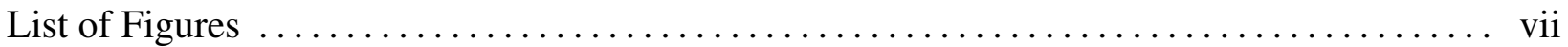

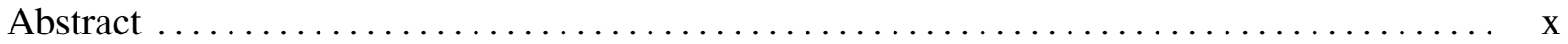

Chapter 1: Introduction $\ldots \ldots \ldots \ldots \ldots \ldots \ldots \ldots \ldots \ldots \ldots \ldots \ldots \ldots \ldots \ldots \ldots \ldots \ldots \ldots \ldots \ldots \ldots, 1$

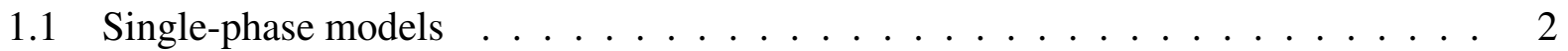

1.2 Two-phase models . . . . . . . . . . . . . . . . 5

1.3 Outline of the dissertation . . . . . . . . . . . . . . . . . . . . 8

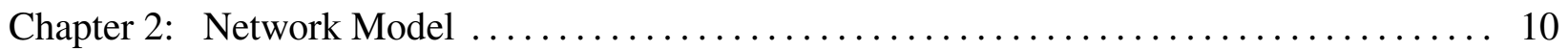

2.1 Network Model Construction . . . . . . . . . . . . . . . . . . . . . . . 10

2.1 .1 Sites (Node Volume) . . . . . . . . . . . . . . . . . . . . 10

2.1.2 Coordination Numbers . . . . . . . . . . . . . . . . . . . . . . . . 12

2.1 .3 Bonds (Conductance) . . . . . . . . . . . . . . . . . . . 13

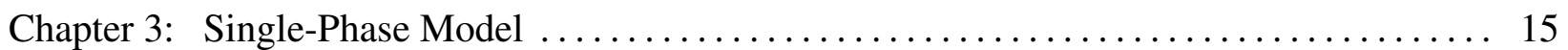

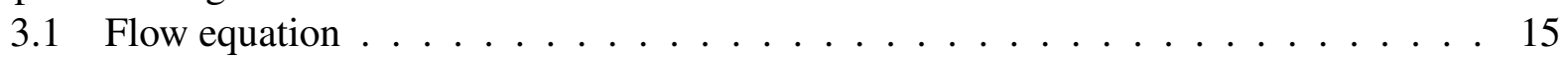

3.2 Optimization . . . . . . . . . . . . . . . . . . . . 17

3.3 Application to synthetic fields . . . . . . . . . . . . . . . . . . . . 19

3.3.1 Homogenous reservoir . . . . . . . . . . . . . . . . . . . . . . . . 19

3.3.2 Presence of barriers . . . . . . . . . . . . . . . . . . . . . 22

3.3 .3 Anisotropy . . . . . . . . . . . . . . . . . 23

3.3.4 Flow paths that act as barriers . . . . . . . . . . . . . . 25

3.3.5 Conductance and permeability . . . . . . . . . . . . . . . . 27

3.3.6 Complex geological features . . . . . . . . . . . . . . . . . . . . 29

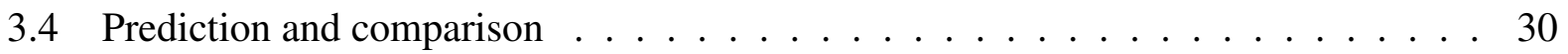

3.4.1 Closed Well . . . . . . . . . . . . . . . . . . . . . . . . . . 30

3.4 .2 Injection fluctuation $\ldots \ldots \ldots \ldots \ldots \ldots$

3.4 .3 Changing flow pattern $\ldots \ldots \ldots \ldots \ldots \ldots \ldots$

3.5 Including additional data $\ldots \ldots \ldots \ldots \ldots \ldots$

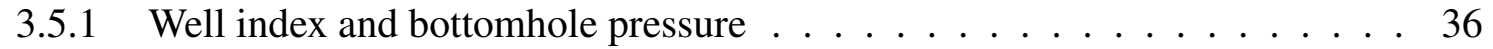

3.5.2 Node volume and compressibility . . . . . . . . . . . . . . . . . 38

3.6 Pressure and saturation dependent properties . . . . . . . . . . . . . . . 40

$3.716 \times 25$ Synthetic Example . . . . . . . . . . . . . . . . . . . . . 45

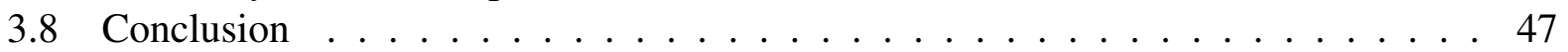

Chapter 4: Single Phase Model with Injector-Injector Interactions $\ldots \ldots \ldots \ldots \ldots \ldots \ldots$

4.1 Including injector-injector intraction in the network model $\ldots \ldots \ldots$. . . . . 51

4.2 Application of the model with injector-injector interaction $\ldots \ldots \ldots 2$ 
4.2.1 Homogeneous case . . . . . . . . . . . . . . . . 52

4.2.2 Directional permeability trend . . . . . . . . . . . . . 54

4.2 .3 Complex geological features . . . . . . . . . . . . . . . . . . . . . . 56

$4.2 .416 \times 25$ Synthetic Field . . . . . . . . . . . . . . . . . 58

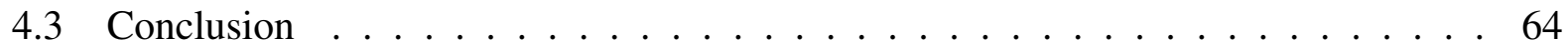

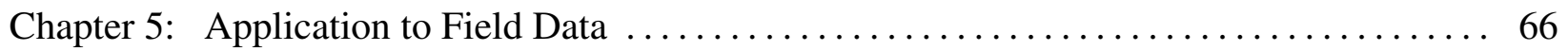

5.1 Open Boundary . . . . . . . . . . . . . . . . 68

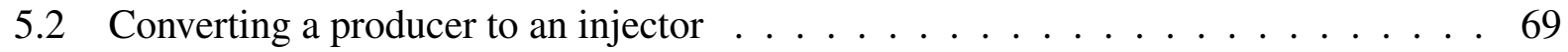

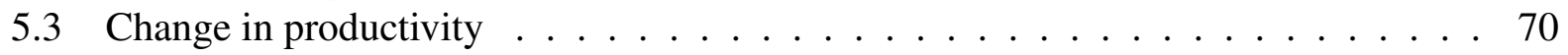

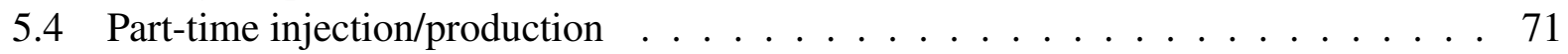

5.5 Results . . . . . . . . . . . . . . . . . . . 72

5.6 Conclusions . . . . . . . . . . . . . . . . . . 74

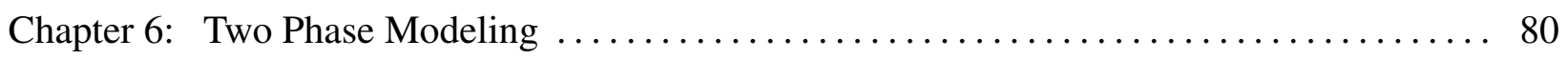

6.1 Two-Phase Flow Equations . . . . . . . . . . . . . . . . . . 80

6.2 Streamlines . . . . . . . . . . . . . . . . . . 82

6.3 Time of Flight . . . . . . . . . . . . . . . . . . . . . . . . . . . . . . . . . . . . . .

6.4 Mapping Saturation along Streamline and Fractional Flow . . . . . . . . . . . . 85

6.5 Two-Phase Flow Parameters . . . . . . . . . . . . . . . . . . . . . . . . . 89

6.6 Limitations of Single Streamline Method . . . . . . . . . . . . . . . . . . 90

6.7 Dykstra-Parsons Method . . . . . . . . . . . . . . . . . . . 91

6.8 Average Fractional Flow at Producers and Interfaces ～. . . . . . . . . . . . . 94

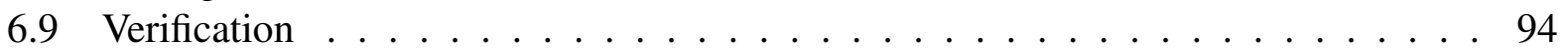

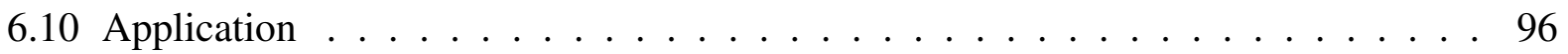

6.11 Conclusion . . . . . . . . . . . . . . . . 106

Chapter 7: Summary, conclusions and future work $\ldots \ldots \ldots \ldots \ldots \ldots \ldots \ldots \ldots \ldots \ldots \ldots \ldots$

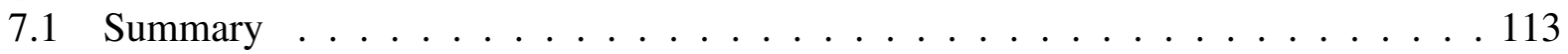

7.2 Conclusions . . . . . . . . . . . . . . . . . . . . 114

7.3 Future work . . . . . . . . . . . . . . . . 115

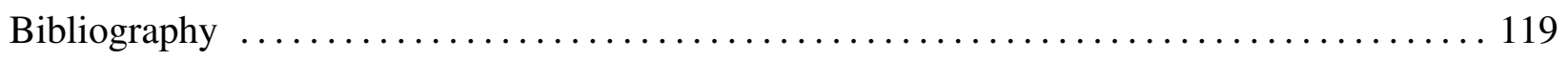

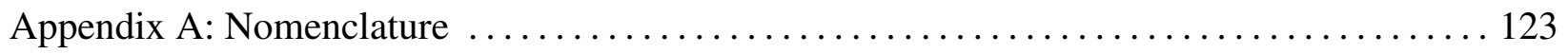

Appendix B: Underdetermined and Overdetermined Problems $\ldots \ldots \ldots \ldots \ldots \ldots \ldots \ldots \ldots$

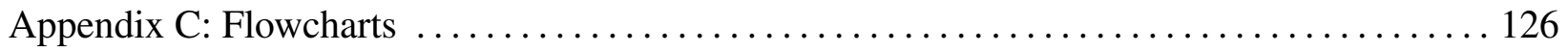

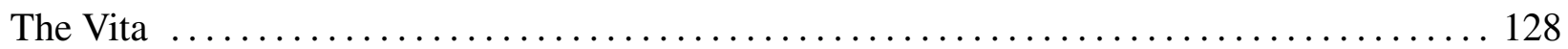




\section{List of Tables}

3.1 Homogenous Reservoir: Conductance $(d . f t / c p) \ldots \ldots \ldots$

3.2 Homogenous Reservoir: Cumulative flow between wells $(b b l) \ldots \ldots$

3.3 Homogenous Reservoir: $k A_{c s}$ values $\left(d . f t^{2}\right) \ldots \ldots \ldots$

3.4 Homogenous Reservoir: Average well allocation factors . . . . . . . . . . . . . . . 21

3.5 Presence of barrier: Conductance $(d . f t / c p) \ldots \ldots \ldots \ldots$

3.6 Presence of barrier: $k A_{c s}$ values $\left(d . f t^{2}\right) \ldots \ldots \ldots \ldots$

3.7 Presence of barrier: Cumulative flow between wells $(b b l) \ldots \ldots$. . . . . . . . . 24

3.8 Presence of barrier: Average well allocation factors . . . . . . . . . . . . . . 24

3.9 Anisotropy: Conductance $(d . f t / c p) \ldots \ldots \ldots \ldots$

3.10 Anisotropy: $k A_{c s}$ values $\left(d . f t^{2}\right) \ldots \ldots \ldots \ldots$

3.11 Anisotropy: Cumulative flow between wells $(b b l) \ldots \ldots$

3.12 Anisotropy: Average well allocation factors . . . . . . . . . . . . . 26

3.13 Yield R-Squared for production prediction when one well is added to the system . . 32

3.14 Yield R-squared values of production match for RM, CM and Network model . . . 34

3.15 Conductance values for a model with known WI $(d . f t / c p) \ldots \ldots$

3.16 Well allocation factors for a model with known WI . . . . . . . . . . . . . 35

3.17 Different BHP at producer $2 \ldots \ldots \ldots \ldots \ldots \ldots$

3.18 Incorrect BHP at producer $2 \ldots \ldots \ldots \ldots$

3.19 Different WI at producer $2 \ldots \ldots \ldots \ldots$

3.20 Incorrect WI at producer $2 \ldots \ldots \ldots$. . . . . . . . . . . . . 40

3.21 Incorrect node volume. . . . . . . . . . . . . . . . . . . . . . 40

3.22 Incorrect compressibility. . . . . . . . . . . . . . . . 41

3.23 Conductance for single phase homogenous reservoir . . . . . . . . . . . . . 43

3.24 Conductance for single phase homogenous reservoir with SPECSP viscosity . . . . 45 
$3.2516 \times 25$ Wells: Conductance $(d . f t / c p) \ldots \ldots \ldots$

$3.2616 \times 25$ Wells: Average well allocation factors $\ldots \ldots$. . . . . . . . . . . 47

4.1 Homogenous Reservoir: Conductance values $(d . f t / c p) \ldots \ldots \ldots$

4.2 Homogenous Reservoir: $k A$ values $\left(d . f t^{2}\right) \ldots \ldots \ldots \ldots$

4.3 Homogenous Reservoir: Cumulative flow between Wells $(b b l) \ldots \ldots 3$

4.4 Homogenous Reservoir: Average well allocation factors . . . . . . . . . . . . . . . 54

6.1 Comparisons of two homogeneous and hetrogeneous linear cases . . . . . . . . . 97

6.2 Obtained parameter values for heterogeneous $2 D$ model $\ldots \ldots$. . . . . . . . . 100

6.3 Obtained parameter values for homogeneous model with an injector and 4 producers. 100

6.4 Obtained parameter values for heterogeneous model with an injector and 4 producers. 100

6.5 Obtained parameter values for $2 D$ model with two injectors . . . . . . . . . . 103

6.6 Time-of-flight Dykstra-Parsons coefficients for the homogenous model from 3.3.1 . 104

6.7 Normalized cross-section area open to flow for the homogenous model from 3.3.1 . 105

6.8 Time-of-flight Dykstra-Parsons coefficients for the homogenous model from 4.2.1 . 105

6.9 Normalized cross-section area open to flow for the homogenous model from 4.2.1 . 105

6.10 Time-of-flight Dykstra-Parsons coefficients for $Y$-direction permeability trend $\ldots 107$

6.11 Normalized cross-section area open to flow for $Y$-direction permeability trend $\ldots 107$

6.12 Time-of-flight Dykstra-Parsons coefficients for high permeability channel $\ldots . .108$

6.13 Cross-section area open to flow for high permeability channel $\left(f t^{2} / f t\right) \ldots \ldots . .108$

6.14 Obtained Saturation in five blocks in the model . . . . . . . . . . . . 110 


\section{List of Figures}

1.1 An example of changes to streamlines as the direction of pressure gradients change. 4

2.1 Schematic of volume assignment to injectors and producers. . . . . . . . . . . 11

2.2 Background grid and Voronoi . . . . . . . . . . . . . . . . . 12

2.3 Node volumes formed by extending Voronoi edges . . . . . . . . . . . . . . 12

3.1 Illustration of the Conductance and flow shown in Table 3.1 and $3.2 \ldots 20$

3.2 Illustration of the conductance and flux shown in Tables 3.5 and $3.7 \ldots 23$

3.3 Illustration of the conductance and flux shown in Table 3.9 and $3.11 \ldots 26$

3.4 Flow paths that act as barriers. . . . . . . . . . . . . . 27

3.5 Change in $k A_{c s}$ as permeability changes in sythetic model . . . . . . . . . . 28

3.6 Two permeability regions in the domain. . . . . . . . . . . . . . . . . . 29

3.7 Distribution of streamlines in the domain $\ldots \ldots \ldots$

3.8 Permeability distribution in the domain $\ldots \ldots \ldots \ldots$

3.9 High permeable channel: Conductance values . . . . . . . . . . . . . . . . . 32

3.10 Injection fluctuation. . . . . . . . . . . . . . . 33

3.11 Permeability distribution in the domain . . . . . . . . . . . . . . 37

3.12 Effect of BHP on conductance values. . . . . . . . . . . . . . . . . 38

3.13 Effect of WI on conductance values. . . . . . . . . . . . . . . . . . 39

3.14 Pressure difference for different relative permeability table. . . . . . . . . . . . . 42

3.15 Pressure difference for different oil viscosity. . . . . . . . . . . . . . . . . . . . 44

$3.1616 \times 25$ wells: Conductance and flux . . . . . . . . . . . . . . . . . 48

4.1 Model parameters for a homogeneous reservoir. . . . . . . . . . . . . . . . . 54

4.2 X-direction permeability trend . . . . . . . . . . . . . . 55

4.3 Y-direction permeability trend . . . . . . . . . . . . . . 56

4.4 Model parameters for X-direction permeability trend. . . . . . . . . . . . . . . 56 
4.5 Model parameters for Y-direction permeability trend. . . . . . . . . . . . 57

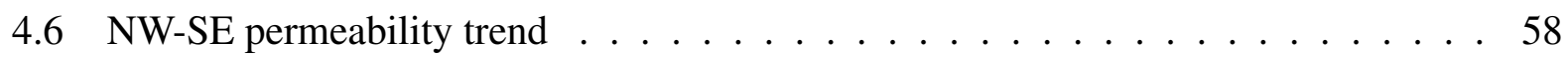

4.7 SW-NE permeability trend $\ldots \ldots \ldots \ldots \ldots$

4.8 Model parameters for NE-SW permeability trend. . . . . . . . . . . . . . 59

4.9 Model parameters for SW-NE permeability trend . . . . . . . . . . . . . . 59

4.10 Omni-direction permeability trend $\ldots \ldots \ldots$. . . . . . . . . 60

4.11 Model parameters for omni-direction permeability trend . . . . . . . . . . . 60

4.12 Model parameters for a high permeable channel with injector-injector interaction. . 61

4.13 Conductance values for the nhomogeneous $16 \times 25$ Synfield. . . . . . . . . . . 62

4.14 Well allocation factor for the homogeneous $16 \times 25$ Synfield. . . . . . . . . . . . 62

$4.15 \mathrm{NW}-\mathrm{SE}$ direction permeability trend in $16 \times 25$ Synfield . . . . . . . . . . . 63

4.16 Conductance values for NW-SE direction permeability trend in the $16 \times 25$ Synfield. 63

4.17 Well allocation factor for NW-SE direction permeability trend in the $16 \times 25$ Synfield. 64

5.1 The study region. . . . . . . . . . . . . . . . . 67

5.2 Voronoi tiles assigned to the wells inside the study region . . . . . . . . . . 68

5.3 Sensitivity analysis on the radius of influence of injectors . . . . . . . . . 69

5.4 Production and injection rates and bottomhole pressure for 900 days study period . 70

5.5 Productivity index vs average daily production rate . . . . . . . . . . 71

5.6 Well allocation factor for equal thickness and equal tile areas . . . . . . . . . 73

5.7 Well allocation factor for pay thickness and Voronoi tile areas . . . . . . . . . . 74

5.8 Out of boundary flow for equal thickness and equal tile areas . . . . . . . . 75

5.9 Out of boundary flow for pay thickness and Voronoi tile areas . . . . . . . . . . 76

5.10 Well allocation factor for equal thickness and equal tile areas . . . . . . . . . 77

5.11 Out of boundary flow and injector-injector allocation factor for equal thickness $\quad \ldots \quad 78$

6.1 Replacing bonds with a diamond shape bonds. . . . . . . . . . . . . . 83

6.2 Representation of the area open to flow and $\Delta s$ along the central streamline path. . . 84

6.3 Flood front has advanced to different distance from injector . . . . . . . . . . 86 
6.4 Moving the saturation forward along streamline by $\Delta t$ step. . . . . . . . . . . . . . 87

6.5 Node volume associated with injector $A$ and producer $B$. . . . . . . . . . . . 90

6.6 Difference between observed fractional flow and model fractional flow. . . . . . . 91

6.7 Dykstra-Parsons cumulative probability plot (Zemel 1995) . . . . . . . . . . . . 92

6.8 Verification of the model with Buckley-Leverett. . . . . . . . . . . . . . 96

$6.91 D$ Model with highly permeable channel. . . . . . . . . . . . . . . . . . 97

6.10 Fractional flow at producer versus dimensionless time for homogeneous case. . . . 98

6.11 Fractional flow at producer versus dimensionless time for the case with channel. . . 98

6.129 layers case: Heterogeneity in vertical direction. . . . . . . . . . . . . . . 99

6.13 Increase in time-of-flight Dykstra-Parsons coefficient with increase in heterogeneity 101

6.14 Reduction of cross section area open to flow with increase in heterogeneity . . . 101

$6.152 D$ Model with diagonal more permeabile region. . . . . . . . . . . . . 102

6.16 Fractional flow at producers versus dimensionless time for $2 D$ model $\ldots$. . . . . 102

6.17 Model with two injector and a high permeability channel. . . . . . . . . . . 103

6.18 The optimum number of streamlines between injector-producer pairs. . . . . . . 109

6.19 Ellipse-shape tube . . . . . . . . . . . . . . . . . 110

C.1 Flowchart showing how single phase network model works. . . . . . . . . 126

C.2 Flowchart showing how two phase network model works. . . . . . . . . . . . 127 


\section{Abstract}

Limited data availability and poor data quality make it difficult to characterize many reservoirs. For reservoirs that have undergone waterflooding, production and injection data are a reliable source of information from which injector-to-producer connections can be inferred. In this research, we use well locations, injection and production rate data and well fractional flow values to develop a reservoir-scale network model.

A Voronoi mesh divides the reservoir into a number of node volumes each of which contains a well. Bonds connect each of the nodes with conductance values that must be inferred from the rate data. An inverse problem is formulated where the mean-squared difference between the modeled and actual production data are minimized and the conductance values between each node are the unknowns. A derivative free optimization algorithm is utilized to minimize the objective function. Knowing the conductivity of each of the bonds, a two phase problem is formulated and solved in this work to obtain the fractional flow at node interfaces and at each producer. The set of cross-sectional areas open to flow and time-of-flight Dykstra-Parsons coefficients that minimize the simulated and actual producer fractional flow values is the solution to this problem.

This work is primarily for secondary and tertiary floods with limited geological data. The solution parameters are directly proportional to formation properties. In addition, they help to evaluate the degree of sweep between wells. This approach has been successfully tested for different synthetic permeability distribution cases and field injection scenarios. The main advantages of the proposed method are:

- It can model changes in flow pattern caused by adding new wells or shutting in producers.

- It uses conventional history matching methods to solve a simplified inverse problem using only production and injection data. It uses a small number of nodes and converges to a better posed solution than statistical approaches. Convergence to a solution for higher frequency data only decreases the speed of the method slightly. 
- The shape of the fractional flow curve is determined by the time-of-flight Dykstra-Parsons coefficients and cross-sectional areas open to flow which can be related to reservoir properties. 


\section{Chapter 1}

\section{Introduction}

Studying the changes in well production rates as injection rates fluctuate, gives insight about interwell formation characteristics. Local permeability values along with the potential gradient, controls the direction of fluid flow in a reservoir. History matching is the most common way of inferring the permeability distribution in a reservoir. History matching involves a cumbersome task of gathering and adjusting numerous data (permeability, porosity, saturation, seismic parameters ...) to match observed and simulated production rates and pressures. Because of the uncertainties associated with the data, detailed output models may not be realistic. Permeability distribution and barriers positions are two examples of reservoir properties and features that are always estimated with uncertainty, unless, there are enough core and well test data available. The simulation model behaves differently in terms of pressure and production response under different realizations of the model data. Therefore, in cases where knowledge of reservoir properties and conditions are limited, models with fewer, well understood parameters may answer questions more confidently than models with many uncertain parameters. For example, the resistance to flow between two points in the medium caused by 1000 block permeability values in a detailed model may be represented by a single parameter in the simple model. Such a parameter may help to reduce the uncertainties of complex models. Even for cases with enough certain data, a detailed answer may not always be necessary and a quick solution may sometimes suffice. In order to obtain such a solution, a simplified model is needed which can be easily set up for different cases. The input to the model should be reliable with limited data processing and the output must have physical meaning.

In this study, simplified models are grouped into two main categories: The first category of models analyze total fluid production and its correlation with injected water. These models usually have a statistical basis and we term these single phase models. The second group of models inspect water-oil-ratio and fractional flow to evaluate waterflood performance and are referred to as two- 
phase models. The dissertation describes new advances in both types and suggests robust methods to analyze single-phase and two-phase production data to infer reservoir heterogeneity.

\subsection{Single-phase models}

Models that are based on the statistical correlation between injection and production rates have been shown to be practically useful to quantify reservoirs that have undergone waterflooding. Many methods have been proposed in this area. Refunjol and Lake (1997) used Spearman analysis and Panda and Chopra (1998) trained an artificial neural network to estimate injector- producer interaction. Albertoni and Lake (2003) implemented multivariate linear regression and diffusivity filters to describe connectivity between injection and production wells. In their model, production rates at a producing well were assumed to be a linear combination of the injection rates of every injector. Diffusivity filters were applied to injection rates to account for potential time lags between injection and the resulting production. In this work this will be referred to as a "resistance model". In order to resolve limitations of the Albertoni and Lake (2003) model, Yousef et al. (2006) proposed a more complex model, named the capacitance model (CM). They incorporated compressibility into this model in addition to transmissibility to quantify the degree of fluid storage between wells. This approach requires bottomhole pressure information in addition to rate data. To improve the CM, Kaviani et al. (2008) proposed a segmented CM for the cases where bottom hole pressure (BHP) data are unknown and a compensated capacitance model when a producer is added or shut-in. Sayarpour (2008) introduced capacitance-resistance models (CRM) in which he solved the fundamental differential equation of the capacitance model based on superposition in time. He used insights gained from performing CRM to reduce the range of input parameters in numerical reservoir simulators.

CRM has been extensively used by authors on many field examples for different purposes. Izgec and Kabir (2009) mentioned the benefits of using CRM compared to solving a transient flow problem. They showed that CRM is applicable even before breakthrough happens and in cases with low injection signal quality. Izgec and Kabir (2010) coupled the CRM and an analytical aquifer model 
to determine the aquifer influx each well receives. Parekh and Kabir (2011) showed that there is agreement between CRM results and interwell tracer data. They also attempted to use CRM and rate-transient analysis to predict connectivity before breakthrough. Wang et al. (2011) used CRM and satellite images of surface subsidence to explain the performance of a waterflood and sugges reasons for the subsidence. They provided a surface subsidence model to predict the average surface subsidence based on the injection and production rates. Bansal and Sayarpour (2012) implemented CRM to estimate fault-block transmissibility. Based on the result, they determined the interaction between compartments.

Dinh and Tiab (2008) also implemented multivariate linear regression, but they used fluctuation of bottomhole pressure of both injectors and producers to calculate inter-well connectivity. Lee et al. (2009) estimated finite-impulse-response (FIR) curves corresponding to the fluid flow between all injector-producer pairs to calculate connectivity between them. In this model, production rates are partly determined by the linear combination of surrounding FIR filtered injection rates.

The recent correlation-based methods generally have the form

$$
q_{j}(n)=\sum_{i=1}^{I} \lambda_{i, j} i_{i, j}^{\prime}
$$

where $\lambda_{i, j}$ is termed the connectivity and is an indicator of the relative contribution that injector $i$ has on production well $j$. The $i_{i, j}^{\prime}$ term is a filtered injection rate. The dissipation of pressure between the injector-producer pair is included in the $i_{i, j}^{\prime}$ term. One of the major deficiencies of such methods is their inability to handle changes in flow pattern (Sayarpour 2008). Change in flow pattern may be a consequence of a long shut-in period for producers or conversion of producers to injectors. Even abrupt changes in injection rates that lead to overpressurizing a region in the reservoir may affect flooding patterns. In these cases the connectivity coefficients are no longer valid. To address this issue, Satter et al. (2007) and Thiele and Batycky (2006) used well allocation factors (WAFs). A well allocation factor is assigned to each injector and is the ratio of the injected fluid volume to the total volume of fluid produced at offset wells (Satter et al. 2007). Thiele and 
Batycky (2006) state that the well allocation factor is a dynamic value, and it changes with injection rate.

Fig. 1.1 shows streamlines generated by FrontSim ${ }^{\circledR}$ software at different times from a simulation of an example problem used by several authors and described in Albertoni (2002). It is a synthetic field where injection rates in 5 wells fluctuate to study connectivity to the 4 producers in the reservoir. Although the flooding pattern is fixed, the influence of each injector on the producers varies as injection rates fluctuate. In the left figure, a high-pressure region forms around injector 1 (I1) caused by a large amount of injection. This causes the streamlines that are launched from injector I3 to avoid this region when flowing towards producers P1 and P2. In the right figure, the region around $\mathrm{I} 2$ is now the high pressure region and the streamlines again avoid the high pressure area when flowing towards producers $\mathrm{P} 1$ and $\mathrm{P} 3$. In each case, injection and production rates in the entire domain and not an individual well, determine the pressure field and the resulting fluid flow direction. Therefore the claim of independency of correlation coefficients from rate may not be appropriate. In addition, parameters estimated by these methods do not have physical meaning, and they cannot be directly used to infer reservoir properties.
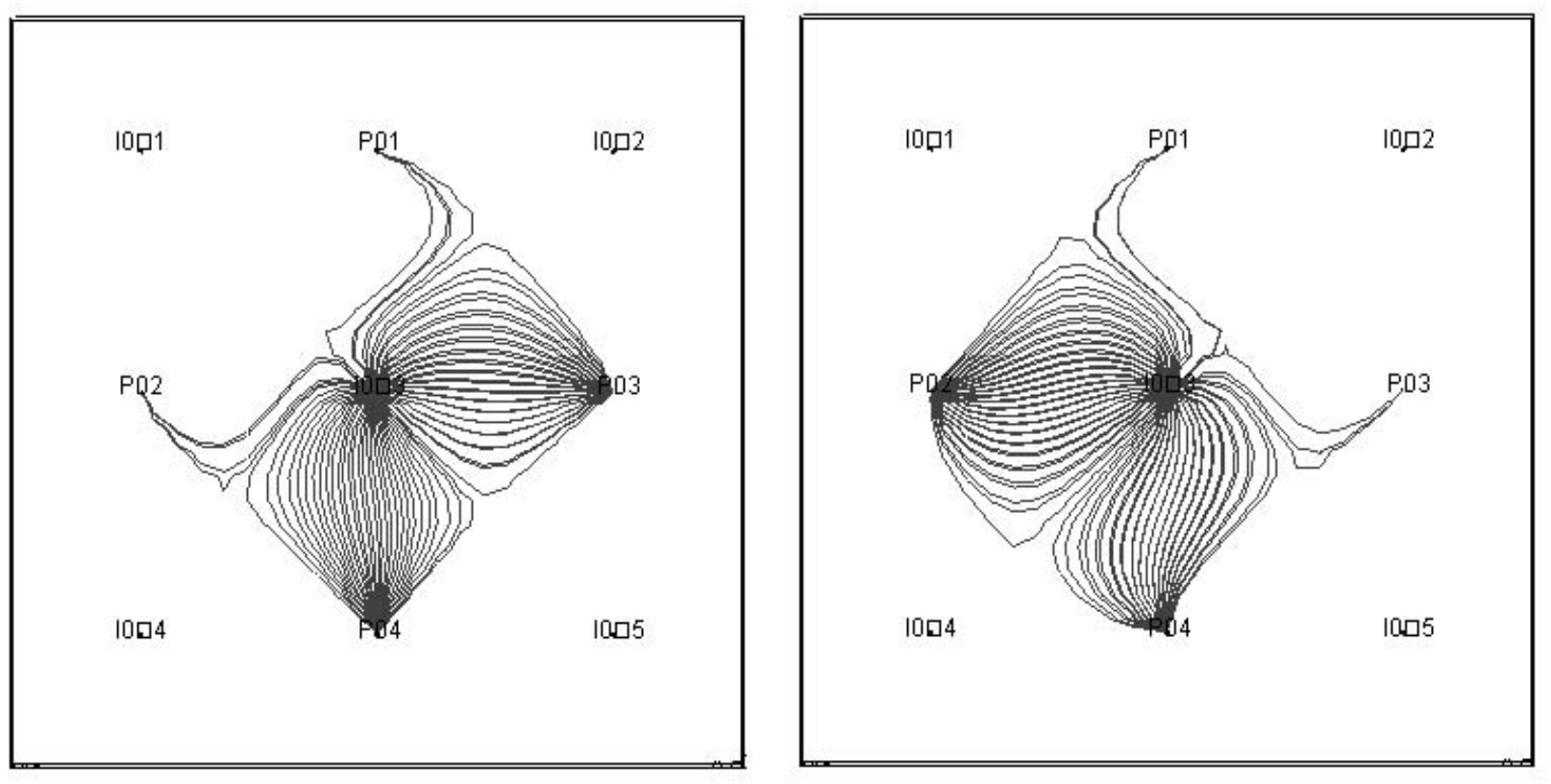

Figure 1.1: An example of changes to streamlines as the direction of pressure gradients change. 
In this research, a tool is developed that uses inverse techniques from automatic history matching, which are simplified such that the numerous grid-block parameters and property characteristics from detailed reservoir characterization are avoided. The amount of input data is reduced to injection and production rates and well positions. Such a parsimonious approach can be applied on reservoirs with minimum data.

The ability to handle changes in flow direction is another characteristic of the model that increases the range of cases that can be studied. Changes in flow direction may be the result of pressurizing certain regions in a reservoir, adding new wells, or shutting-in producers. Permeability and pressure gradient determine flow direction in a reservoir. Since the approach is based on a simulation flow equation, permeability and pressure gradients are included. Therefore it can handle changes in flow direction.

The method is capable of matching one or selected time intervals of production history for an entire field or group of wells. It estimates parameters that can be used to infer permeability variations and geological features in the reservoir. In addition, once parameters are estimated the model can be converted to a predictive tool to estimate total production rates in each producer using injection data as input. Different case studies with different permeability distributions and/or injection-production scenarios are considered to test the performance of the method under different conditions.

\subsection{Two-phase models}

The importance of characterizing heterogeneity in the reservoir cannot be overstated since good sweep efficiency and as a result, success of the displacement is obtained when there is a good understanding of the heterogeneity in the reservoir. Although conductance values give an indication of the direction of fluid flow, seals and high permeable pathways, they are incapable of demonstrating the spatial variations of physical parameters in the reservoir. This is one of the weak points of the connectivity values in the statistical methods as well. Having the heterogeneity knowledge and implementing flood-optimization techniques will help to improve volumetric sweep efficiency. 
There are many analytical and semi-analytical methods in the literature to analyze and forecast flood performance. These methods usually use the well fractional flow values and do not deal with reservoir heterogeneity. Plotting the water-oil ratio (WOR) versus cumulative-oil production $\left(N_{p}\right)$ on a semi-log scale was proposed by Arps (1945), and a crossplot technique proposed by Ershaghi and Abdassah (1984) are two of these methods. The crossplot is based on the linear relationship between the logarithm of the relative permeability ratio and water saturation and the BuckleyLeverett equation. Yang (2009) used the same assumption of a semi-log relationship between the oil-water relative permeability ratio and water saturation and derived an analytic solution that relates oil fractional flow and volumetric sweep efficiency. Can and Kabir (2012) improved on Yang's model by correcting its performance at high WOR.

Kabir and Izgec (2009) tried to identify high conductivity layers or channels between injector and producer pairs using a modified-Hall analysis. The modified-Hall plot uses an improved Hall integral curve and two derivative curves. Normally, the derivative curves match the integral curve during matrix injection, but fall lower when fracturing occurs. Kabir and Izgec (2009) transformed the separation distance between the derivative curves and the integral curve into a permeabilitythickness product.

To enhance inference about reservoir heterogeneity, Yousef et al. (2009) realized that heterogeneity cannot be inferred from the resistance and capacitance parameters in the CM model individually. They combined both sets of parameters to redefine a flow capacity plot and a Lorenz plot. Then, they used the plots to identify whether the connectivity of an injector-producer well pair was through fractures, a high permeability layer, multiple layers, or through a partially completed well.

Lee et al. (2008) tried to identify high-permeability channels based on signal-processing techniques applied to only injection and production rates. Like Yousef et al. (2009), they used flow capacity plots. They obtain their flow capacity plots from finite-impulse-response (FIR) curves corresponding to the fluid flow between an injector-producer pair.

Although reservoir heterogeneity has higher influence on two-phase flow behavior like fractional flow and breakthrough time compared to total production, neither Yousef et al. (2009) nor Lee et al. 
(2008) looked at individual phase production. Liang et al. (2007) and Sayarpour et al. (2007) tried to optimize waterflood performance using an empirical oil fractional flow model. The model is based on a power-law relationship between the instantaneous water-oil ratio and the cumulative water injected. The model has two parameters, $a$ and $b$, which are used to match oil fractional flow but are not reservoir parameters.

In this thesis, individual phase rates will be analyzed in addition to the total rates to reveal more information about interwell characteristics. More specifically, the water fractional flow that each injector brings to a producer needs to be determined. This should provide an idea of the degree of heterogeneity and sweep between the well pairs, since the shape of the fractional flow curve is a function of the heterogeneity between wells. This requires having an oil-production model that matches average fractional flow that each injector brings to the producer with the observed fractional flow. In a reservoir with multiple injection wells, it would be difficult to obtain a realistic fraction of the flow from each injector. What is observed at a producer is one fractional flow function. There may be many combinations of cases that provide the observed fractional flow behavior at the producer.

The two-phase flow model that is proposed in this study is a conventional two-phase flow model that is implemented on a network representing the reservoir. In two-phase flow models, front position and saturation distribution are functions of permeability and reservoir heterogeneity. These are key parameters when it comes to matching the fractional flow at producers and are typically determined by changing grid properties in traditional history matching techniques. In this thesis, the assumption is that the geological information is limited, and therefor there is no underlying geologic model to match to. Instead, ideas from streamline simulation and saturation mapping are used to track fronts and obtain saturation values between injector-producer pairs. Unlike streamline simulation, where an underlying grid dictates the front and saturation along each streamline, in this work, a cross-sectional area open to flow and a "Dykstra-Parson" coefficient are used to characterize the fluid movement. The cross-sectional area open to flow gives an average time of flight in a diamond-shaped bond that connects each injector-producer pair. Within this bond "streamlines" 
are launched from the injector, and a factor similar to a Dykstra-Parsons coefficient is used to represent the heterogeneity between the injector and the producer and determines the distance that fronts advance in each streamline.

Another difference between traditional streamline simulation and the network model is the saturation assignment to the domain. Both methods are Implicit in Pressure, Explicit in Saturation methods (IMPES). That means both solve a pressure equation and then a saturation equation. In both, a $3 D$ saturation equation is transformed into a $1 D$ equation along a flow direction. In streamline simulation, the saturation of the grid blocks in the system are calculated by averaging the saturation in the streamlines that pass through the blocks. In the network model, a fractional flow value between node volumes is used to find a representative relative conductivity of each phase between node volumes. These relative conductivity values are used in the saturation equation to find the saturation of the node volumes. Therefore, there are two saturation equations: one is a pseudo- $1 D$ equation within each bond that finds the relative conductivity values and the second is a saturation equation that updates the node volume saturations. The reason for doing this is to assign consistent saturations to the node volumes and allow an estimation of the unswept volume in each node.

\subsection{Outline of the dissertation}

The dissertation consists of seven chapters. Chapter 1 explains the motivation for this study and reviews previous attempts to characterize reservoirs through rate analysis.

Chapter 2 discusses network modeling basics and how it is applied to a larger scale. The singlephase flow equation and the optimization method are discussed in Chapter 3.

In Chapter 4, the method is applied to synthetic cases to test the ability of the method to find reservoir features that control fluid flow.

Application of the method to field data is presented in Chapter 5. In this chapter, the problems associated with application of the method to field data are discussed. 
Chapter 6 presents the extension of the method to analyze multiphase production and gain more insight about reservoir heterogeneity.

Chapter 7 gives the summary and conclusions that follow from this dissertation and provides recommendations for future research work in this area. 


\section{Chapter 2}

\section{Network Model}

In the approach developed in this work, the reservoir is viewed as a network model consisting of sites and bonds. In standard pore-network modeling, sites are equivalent to pore bodies and bonds to pore throats. In this work, the sites correspond to the volumes assigned to each well, and bonds to the connection between wells. This assignment is different from how Samier et al. (2001) associated pore volume to each well. They used a streamline simulator and detailed reservoir characterization and found the geometric influence zone around each injector and producer based on the generated streamlines. The influence zone of injectors and producers may overlap but may not cover the whole reservoir.

To construct the model presented in this work, the macro-scale geometry of the reservoir and well positions are required. The relative distance between the wells and the distance to any of the boundaries determine the volume size rather than flow characteristics of the domain. In addition, the model covers the whole reservoir. First, characteristics of the model are discussed. Then a flow equation and an optimization method will be developed to find the unknowns in the model.

\subsection{Network Model Construction}

This network model, as other network models, is characterized by three properties:

- Sites (the node volume or the volume assigned to each well)

- The network coordination number (the number of bonds attached to each site)

- Bonds (conductance)

\subsubsection{Sites (Node Volume)}

The principal objective here is to represent the domain of a reservoir by a set of tiles each of which contains a well. Each tile has a known surface area and a thickness corresponding to the thickness 
of the reservoir in the neighborhood of the well. Each tile then represents a node volume and is referred to as $V_{b}$ in the calculations (Figure 2.1). Later, it will be explained that if the reservoir structure is available, it can be used to find the tile thicknesses. The summation of volumes should be equal to the estimated volume of the reservoir. Since the intention is to work with the communicating parts of the reservoir, multiplying the tile areas by net pay results in more realistic node volumes. This volume is constant during the study period and is not a function of rate variations. Therefore it is the true drainage volume of the well only in the case of a homogeneous system with homogeneous rate variations.

In this work, Voronoi tessellation is used to divide the reservoir area into convex polygons. The tiles have a property that any point in the interior of the tile is closer to the well at the center of the tile than to any other well (Farrashkhalvat and Miles 2003). Voronoi cell generation requires a background grid that is generated by the Delaunay method. The area of the reservoir is the triangulation domain, and well positions are input nodes. Tiles are formed by connecting the circumcenters of the Delaunay triangles. As shown in Figure 2.2, Voronoi cells that are generated only using well positions cannot cover the entire area of the reservoir. The boundaries of the reservoir that are not covered by the triangles must be included in order to calculate realistic node volumes. To include

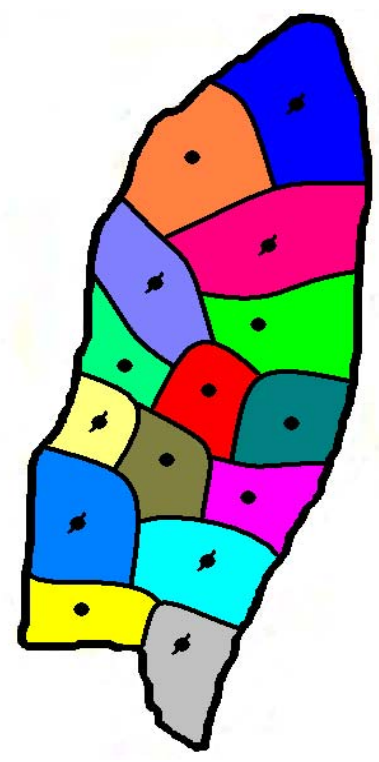

Figure 2.1: Schematic of volume assignment to injectors and producers. 
the uncovered areas, the edges of the Voronoi grid are extended to intersect the boundaries. The area surrounded by the boundary of the reservoir and the Voronoi edges are used to calculated node volumes for boundary nodes (Figure 2.3).

\subsubsection{Coordination Numbers}

In porous media, fluid flows along various throats that are connected to each pore. The number of throats connected to each site is called the coordination number $Z$. With different values of $Z$, the topology of the porous media can be well defined.

In this work, the coordination number is defined by the number of production wells connected to an injection well. It is the number of production wells that are influenced by an injector well. In

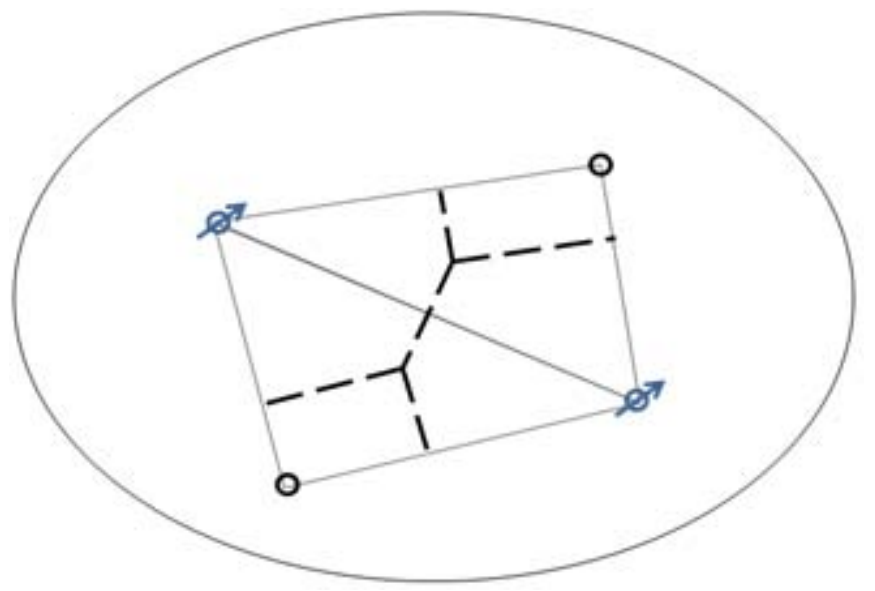

Figure 2.2: Background grid and Voronoi formed by connecting the center of triangles.

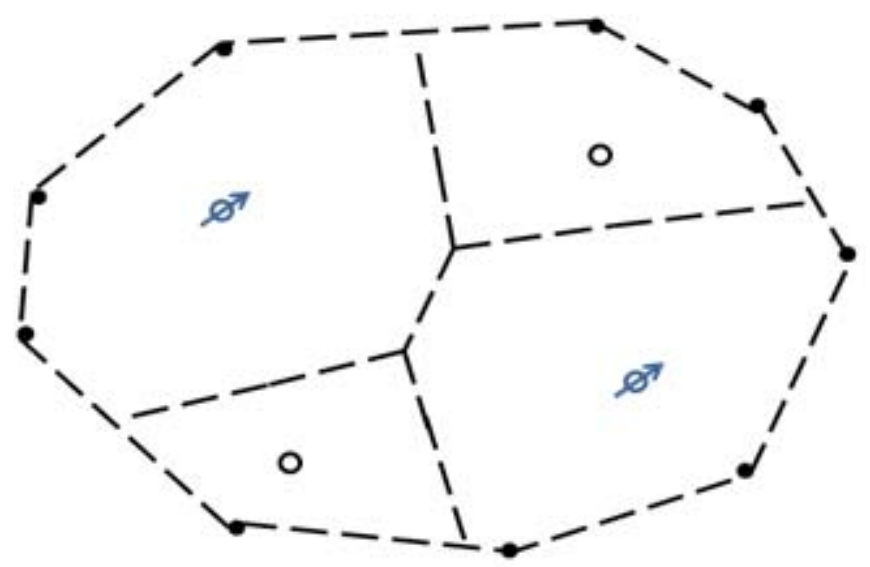

Figure 2.3: Node volumes formed by extending Voronoi edges 
this portion of the work injector-injector and producer-producer connections were initially ignored. The question was, which producing wells are influenced by which injection wells? Obviously, producers likely receive larger volumes from close injectors in homogeneous formations.

Producers that do not have a bond with an injection well are not affected by it. So the more bonds an injection well has, the more likely it will be that distant injector-producer connections that may occur in heterogeneous reservoirs will be seen. Increasing the number of bonds increases the number of unknowns, which consequently increases computational time in the optimization. So there is a tradeoff between the number of bonds and the speed with which a solution is obtained.

To determine the coordination number of an injector, an influence radius can be assigned to it. Then, those producing wells that fall within a circle with that radius of influence centered on the injection well are assigned bonds with the injector. For problems with a small number of wells, all injectors are connected to producers. For larger problems, this radius should be determined and may be directional.

Permeability and porosity of the rock and compressibility of the fluid are the main properties that control the radius of influence (Lee 1982); since these terms are initially unknown, distance between an injector and a producer is the only parameter that can be used to determine the appropriate radius of influence distance for each connection. Kaviani et al. (2010) adressed a model reduction approach called windowing, which is very similar to what is being done in this work. They used the location of wells to define the window.

\subsubsection{Bonds (Conductance)}

Like the role of a throat in pore network modeling, a bond connects node volumes and controls transmissibility between the nodes. In other words, they act as the conduits for fluid flow between injection and production wells. In the pore network modeling literature, this conduit is designated as the conductance between the centers of pore bodies and is denoted $g_{I J}$ (Bakke and Øren 1997). The subscripts denote node volumes $I$ and $J$. The volumetric flow rate between two connected 
nodes $I$ and $J, q_{I J}$ is given by

$$
q_{I J}=g_{I J}\left(p_{I}-p_{J}\right)
$$

In this approach $q_{I J}$ represents the flow rate between the connected injector and producer, $i$ and $j$. For Darcy flow between wells, $g_{I J}$ will be:

$$
g_{I J}=\frac{k_{I J} A_{c s I J}}{\mu L_{I J}}
$$

Where $k_{I J}, A_{c s I J}$, and $L_{I J}$ are the average permeability, area open to flow, and conductance path distance between nodes (wells), respectively, and are all properties of the domain. $\mu$ is the viscosity and is a fluid property. Since the problem considered here is a two-phase flow system, $\mu$ will be the average viscosity of the two phases. Note also that capillary pressure and gravity are ignored in this formulation.

Although distance between wells is a reasonable value for the $L$ in the conductance formula, it may not be representative of the actual path that streamlines travel since it would be the shortest streamline distance between wells. For homogeneous cases this may be a good approximation, but for cases with complex features it likely underestimates the distance.

In this work, it is assumed that $A_{c s}$, or the cross-sectional area open to flow, is obtained by multiplying the length of the "common edge" of Voronoi cells between adjacent injectors and producers by their average thickness values. This area is not a good representative of the area open to flow. First of all, there is no "common edge" between nonadjacent cells (wells), despite the fact that there may be connection between them. For homogeneous cases, cross-sectional area open to flow depends on the flux between wells, and the flux varies with fluctuations in rate. For nonhomogeneous cases with geologic complexity, the area term may be far different from what is assumed. The impact of these formulation "errors" will be shown to be minimal to the evaluation of interwell connectivity.

In this way, properties between an injector-producer well pair are assigned to the bond that connects corresponding nodes in the network model. 


\section{Chapter 3}

\section{Single-Phase Model}

In this network model, a volume is assigned to each well, and the summation of these volumes is equal to the total volume of the reservoir. Conductance values of bonds that control flow in the domain are unknowns and need to be determined. Given proper values of conductance the model response due to the stimulus would be the same as that of the real case, where the stimulus is the change in injection rate, and the response is the consequent change in production. In other words, optimal values of conductance minimize the squared difference between model and observed production rates. In this chapter, flow equation and problem set up for a single phase model will be discussed, and then the approach will be verified be several synthetic examples.

\subsection{Flow equation}

The continuity and Darcy equations are combined, as suggested by Ertekin et al. (2001), to obtain flow equations for the network model. For producer and injector flow, these equaitons may be written as:

$$
\begin{aligned}
& \sum_{I=1}^{Z_{j}} \beta_{c} \frac{k_{I J}}{B \mu} \nabla p_{I J} A_{c s I J}+q_{s c}=\left(\frac{V_{b} \phi c}{\alpha_{c} B^{0}} \frac{\partial p}{\partial t}\right)_{J} \\
& \sum_{J=1}^{Z_{i}} \beta_{c} \frac{k_{I J}}{B \mu} \nabla p_{I J} A_{c s I J}+q_{s c}=\left(\frac{V_{b} \phi c}{\alpha_{c} B^{0}} \frac{\partial p}{\partial t}\right)_{I}
\end{aligned}
$$

$I$ represents the node volume containing injector $i$, and $J$ represents the node volume containing producer $j$. Porosity, $\phi$, is assumed constant, but $B$, the formation volume factor (FVF), $c$, the compressibility, and $\mu$, the viscosity, are pressure dependent. $Z_{j}$ and $Z_{i}$ are the producer and injector coordination number, $\alpha_{c}$ and $\beta_{c}$ are volumetric and transmissibility unit conversion factors, and $B^{0}$ is the FVF at a reference pressure. $V_{b}$ is the bulk volume of the node and is obtained by multiplying the node (tile) area by its average thickness. $A_{c s I J}$ is the cross-sectional area open to flow between 
nodes (wells) $I$ and $J$ and is different from the node or tile area. $\nabla p_{I J}$ is the pressure gradient, and $k_{I J}$ is the average permeability between injector $i$ and producer $j$ that correspond to nodes $I$ and $J$. For a particular time discussed as $n+1$, the pressure gradient is estimated by

$$
\frac{p_{J}^{n+1}-p_{I}^{n+1}}{L_{I J}}
$$

$P$ is the node pressure and $L$ is assumed to be the distance between the wells. Substituting the pressure gradients into the flow equations gives:

$$
\sum_{I=1}^{Z_{j}} \beta_{c}\left(\frac{1}{B^{n} \mu^{n}}\right)\left(\frac{A_{c s I J}}{L_{I J}}\right) k_{I J}\left(p_{J}^{n+1}-p_{I}^{n+1}\right)+q_{s c}=\left(\frac{V_{b} \phi c^{n}}{\alpha_{c} B^{0}}\right)_{J} \frac{p_{J}^{n+1}-p_{J}^{n}}{\Delta t}
$$

for producers and

$$
\sum_{I=1}^{Z_{i}} \beta_{c}\left(\frac{1}{B^{n} \mu^{n}}\right)\left(\frac{A_{c s I J}}{L_{I J}}\right) k_{I J}\left(p_{J}^{n+1}-p_{I}^{n+1}\right)+q_{s c}=\left(\frac{V_{b} \phi c^{n}}{\alpha_{c} B^{0}}\right)_{I} \frac{p_{I}^{n+1}-p_{I}^{n}}{\Delta t}
$$

for injectors. After substituting $g_{I J}$ in Eq. 3.4 and Eq. 3.5 they become:

$$
\begin{aligned}
& \sum_{I=1}^{Z_{j}} \beta_{c}\left(\frac{g_{I J}}{B^{n}}\right)\left(p_{J}^{n+1}-p_{I}^{n+1}\right)+q_{s c}=\left(\frac{V_{b} \phi c^{n}}{\alpha_{c} B^{0}}\right)_{J} \frac{p_{J}^{n+1}-p_{J}^{n}}{\Delta t} \\
& \sum_{I=1}^{Z_{i}} \beta_{c}\left(\frac{g_{I J}}{B^{n}}\right)\left(p_{J}^{n+1}-p_{I}^{n+1}\right)+q_{s c}=\left(\frac{V_{b} \phi c^{n}}{\alpha_{c} B^{0}}\right)_{I} \frac{p_{I}^{n+1}-p_{I}^{n}}{\Delta t}
\end{aligned}
$$

The superscript $n$ indicates the time step at which parameters are evaluated. $q_{s c}$ is a source when the equation is written for a producer and a sink when it is written for an injector. In conventional reservoir simulation, $q_{s c}$ is related to the well index, $J_{w}$, and the difference between average block pressures and flowing sandface pressures, $p_{w f}$. In the network model, injection rates are provided and fixed, and production rates are unknowns to be determined by an optimization procedure. The injectivity and bottomhole pressure response of injectors is dampened by the node volumes. Thus the well index for injectors is of minor importance compared to those of producers that control the fluctuating production rates in the producers. Production is a match parameter and is related to the bottomhole pressure in a well by:

$$
q_{s c}=-J_{w}\left(p_{J}-p_{w f}\right)
$$


For a case of unknown well index and bottomhole pressure, a large value can be assigned to $J_{w}$ to reduce the effect of the well, and average reservoir pressure is used for $p_{w f}$. It is also assumed that for production wells, $p_{w f}$ values are constant. If $p_{w f}$ values are known at various times, these values can be used as additional constraints in the optimization procedure.

\subsection{Optimization}

In its most general form, an inverse problem refers to the determination of the plausible physical properties of a system, or information about those properties, given the observed response of the system to some stimulus (Oliver et al. 2008). For this work, the inverse problem is the problem of determining the connectivity between wells from production data measured at producing wells. Connectivity is the model parameter. The choice of model parameter refers to parameterization of the system (Oliver et al. 2008). For the network model, injection well rates provide the stimulus, and producing well rates represent observed data. The model is the relationship between the observed data and the model parameters.

Inverse problems are typically ill posed; many good solutions exist that are consistent with the observed data and constraints of the system. For the network model problem, production and injection measurements are subject to noise, so measured data may not be exact. The model also may not be exact, since model assumptions may not be consistent with reality. In such cases several solutions may be considered a good solution. In this case prior information for the model may be helpful. As mentioned above, a solution to the problem should be consistent with the observed data and constraints of the system. To obtain a good solution the difference between observed data and the modeled response must be minimized. In this case, one has to minimize the difference between the observed production and the modeled production response using estimated connectivity values. This goal is obtained by minimizing the objective function:

$$
F(g)=\frac{1}{2}\left(q_{m o d}(g)-q_{o b s}\right)^{T} C_{D}^{-1}\left(q_{m o d}(g)-q_{o b s}\right)
$$


where $q_{m o d}$ is an assumed theoretical model for predicting production for a given $g, q_{o b s}$ is a $N_{D}$ dimensional column vector containing measured production values, $C_{D}$ is a $N_{D} \times N_{D}$ covariance matrix for data measurement and modeling errors, and $g$ is an $N_{m}$-dimensional collumn vector of model variables. $N_{m}$ and $N_{D}$ are the number of model variables and the number of observations, respectively.

To minimize Eq. 3.9 a derivative-free alogorithm is implemented. The derivative free algorithm used in this context is designed for least-square problems of the form:

$$
\min F=\frac{1}{2} \sum_{n=1}^{N_{D}} f^{n 2}
$$

where $f^{n}, n=1, \cdots, N_{D}$ are generally nonlinear functions. If the $C_{D}$ matrix is defined as a diagonal matrix, $f^{n}$ can be written as:

$$
f^{n}=q_{m o d}{ }^{n}-q_{o b s}{ }^{n} \quad n=1, \cdots, N_{D}
$$

For the nework model, $f^{n}$ is the difference between observed and model production rates, at time $n$, and the function value is extracted from simulation. Eq. 3.11 is written based on the assumption that the components of the data error vector are independent Gaussian random variables with all means equal to zero and variance $\sigma_{d}$.

The difficulties associated with calculating a sensitivity matrix makes it difficult to compute first- and second-order derivatives of the objective function and it is more desirable to treat the function as a "black box", necessitating that derivative-free algorithms be used. In addition, since the objective function value may be computed with some noise, the finite difference estimation of the derivative may not be accurate. The goal of these algorithms is to solve Eq. 3.10 with the least possible number of function evaluations (Zhang et al. 2010).

In this work, a class of the derivative-free method that requires the sequential minimizations of models is implemented. The minimizations are quadratic or linear and are based upon evaluations of the objective function at sample sets (Zhang et al. 2010). 


\subsection{Application to synthetic fields}

This section examines the application of the technique on a numerically simulated synthetic field. This case was presented by Albertoni and Lake (2003) and has been used by many authors to study well connectivity relationships. The domain is a single-layered reservoir with a closed boundary. A five-spot injection pattern is used to investigate the performance of the method. The case has 5 injectors, 4 at the "corners" of the domain and one in the middle. The 4 producers on the top, bottom, left, and right sides of the domain establish the injection pattern. Oil and water compressibility values are both set to $5 \times 10^{-6} \mathrm{psi}^{-1}$, and rock compressibility is ignored. All wells are vertical, and adjacent wells are distanced $800 \mathrm{ft}$ apart. The injection data from Albertoni and Lake (2003) are used, as stimulus and production is controlled by the injection rates, since BHPs are equal and fixed at 500 psi. The numerical simulator runs for 100 months with $\Delta n=1$ month. Different permeability distributions and injection senarios are applied to the domain to test the performance of the method under different interwell connection cases.

\subsubsection{Homogenous reservoir}

The first case is a homogenous system with an isotropic permeability of $40 \mathrm{md}$. The numerical values of conductance and fluid flow between injector-productor well pairs are shown in Table 3.1 and Table 3.2 respectively. Fluid flow between the well pairs is the amount of injection from an injector that influences production in a producer. Once conductance values are calculated, fluid flow is computed by multiplying values of the conductance times the pressue gradient between the wells. Conductance and flow values are shown in Figure 3.1. Conductance is represented by the thickness, and flow by darkness of the bonds. The thicker the bond, the larger the value of conductance, and the darker the bond, the larger the volume of fluid that has flowed through the bond.

Figure 3.1 indicates both reservoir and flow properties. Thicker bonds between closer well pairs indicates higher conductance between them. That makes sense, since conductance is inversely related to the distance between wells when permeability is homogeneous. For those wells that are 
Table 3.1: Homogenous Reservoir: Conductance (d.ft/cp)

\begin{tabular}{ccccc}
\hline & P1 & P2 & P3 & P4 \\
\hline I1 & 0.271 & 0.273 & 0.130 & 0.124 \\
\hline I2 & 0.254 & 0.134 & 0.266 & 0.159 \\
\hline I3 & 0.201 & 0.182 & 0.202 & 0.196 \\
\hline I4 & 0.159 & 0.289 & 0.152 & 0.272 \\
\hline I5 & 0.123 & 0.121 & 0.240 & 0.239 \\
\hline
\end{tabular}

Table 3.2: Homogenous Reservoir: Cumulative flow between wells $(b b l)$

\begin{tabular}{ccccc}
\hline & P1 & P2 & P3 & P4 \\
\hline I1 & 58676 & 59401 & 28364 & 27086 \\
\hline I2 & 42021 & 22315 & 44361 & 26713 \\
\hline I3 & 23838 & 21769 & 24240 & 23726 \\
\hline I4 & 14560 & 26793 & 14135 & 25535 \\
\hline I5 & 17535 & 17393 & 34493 & 34581 \\
\hline
\end{tabular}

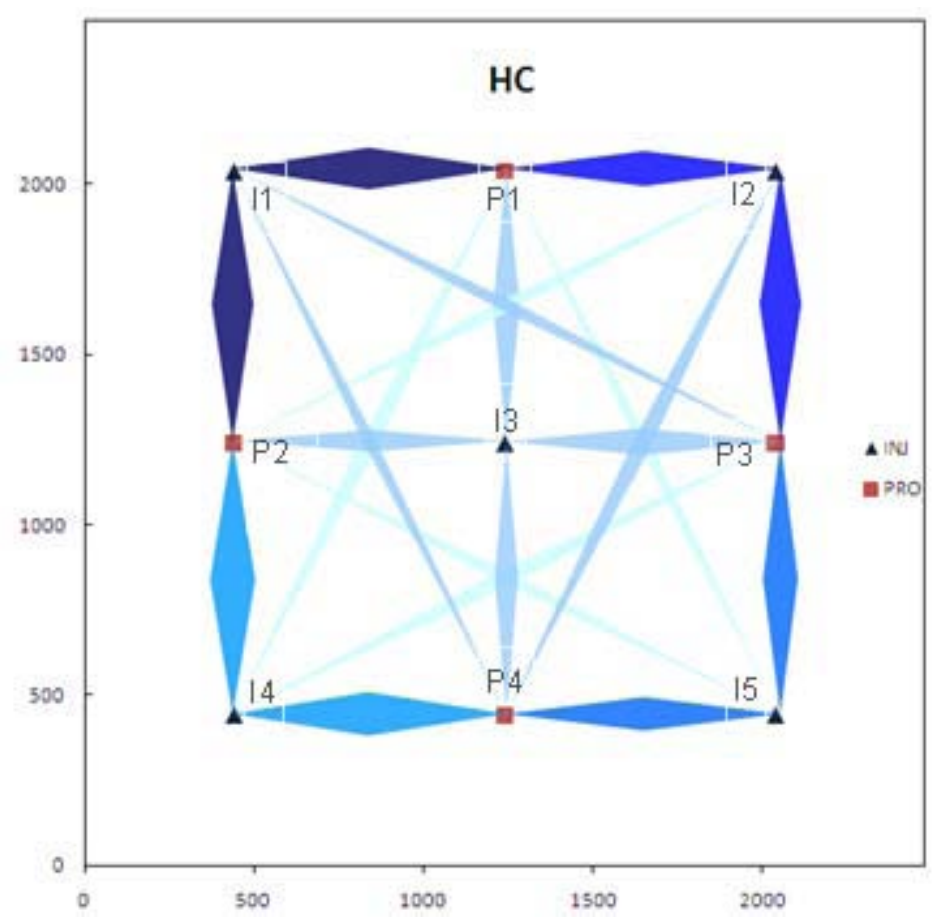

Figure 3.1: Illustration of the Conductance and flow shown in Table 3.1 and 3.2 
equally distanced apart, thicker shapes correspond to a higher $k A_{c s}$ value. From Figure 3.1 it can be inferred that flow is directly related to $g$, since the thicker the bond, the darker it becomes. That makes sense also, since flow is directly proportional to $g$. This explains why the closer the wells are, the larger amount of fluid flow between them. The darkness of bonds connected to an injector also depends on the amount of water injected, which in this case is the darkness is consistent with injection rates.

Table 3.3 shows $k A_{c s}$ values, which are obtained by dividing the conductance by the distance between wells. Average well allocation factors are presented in Table 3.4. The average well allocation factors are the fractions of water injected that influence production in a producing well (Thiele and Batycky 2006). In other words, it is obtained by dividing values of each row of Table 3.2 by the sum of values of that row.

Table 3.3: Homogenous Reservoir: $k A_{c s}$ values $\left(d . f t^{2}\right)$

\begin{tabular}{ccccc}
\hline & P1 & P2 & P3 & P4 \\
\hline I1 & 216.9 & 218.6 & 233.1 & 221.7 \\
\hline I2 & 203.1 & 239.7 & 212.8 & 285.0 \\
\hline I3 & 160.5 & 145.4 & 161.5 & 156.9 \\
\hline I4 & 283.9 & 231.1 & 272.0 & 217.6 \\
\hline I5 & 220.4 & 217.0 & 192.2 & 191.5 \\
\hline
\end{tabular}

Table 3.4: Homogenous Reservoir: Average well allocation factors

\begin{tabular}{ccccc}
\hline & P1 & P2 & P3 & P4 \\
\hline I1 & 0.338 & 0.342 & 0.163 & 0.156 \\
\hline I2 & 0.310 & 0.165 & 0.328 & 0.197 \\
\hline I3 & 0.255 & 0.233 & 0.259 & 0.254 \\
\hline I4 & 0.180 & 0.331 & 0.174 & 0.315 \\
\hline I5 & 0.169 & 0.167 & 0.332 & 0.333 \\
\hline
\end{tabular}


One of the important characteristics of Albertoni and Lake (2003) and Yousef et al. (2006), as they pointed out in their papers, is the estimation of symmetric values for connectivity. They concluded that symmetry indicates that weights do not depend on injection rate. In their work, connectivity weight, which is the fraction of injection rate that goes to a producer, shows a symmetric behavior, but the time constant, which includes productivity and pore volume of the producer, is not as perfectly symmetric. In this work, the well allocation factors obtained are symmetric, but conductivity values are not as symmetric as well allocation factors. Thus it can be concluded that conductance values depend on injection rates. Since $k$ is constant and homogeneous and the wells are equally spaced ( $\mathrm{L}$ values are constant), the only property that the calculated rate depends on should be $A_{c s} . A_{c s}$ is defined to be the area open to flow, or in other words, a cross section of the reservoir between the injector and producer well pair. One can compare $A_{c s}$ values with the number of streamlines between well pairs. In a homogeneous case the number of streamlines between a well pair and the area covered by them is proportional to the flow between the wells. When injection rates fluctuate, the flow between the wells and the number and area covered by streamlines change as well. Therefore, one may conclude that $A_{c s}$ values are proportional to the flow and change as the rate fluctuates. As a result, one expects that conductance values between injector I1 and producers to be larger than conductance values of injector I4 that has lower injection rates. But the values of Table 3.2 do not confirm this hypothesis. Values of $k A_{c s}$ for injection I4 are relatively higher than other injectors, in spite of higher injection rates in injector I1. The smaller pressure gradient between injector I4 and producers may be the reason for higher conductance values around I4. Table 3.4 also shows good symmetry. Injector I 2 shows an asymmetric behavior, since injection in injector I1 is comparatively higher than in I4. Therefore flow goes toward the producers with less support.

\subsubsection{Presence of barriers}

The method can recognize the introduction of a sealing fault into the model. A sealing fault in the Albertoni and Lake (2003) homogeneous system was modeled. The sealing fault was created by 
setting the transmissibility multiplier to 0 between certain grid cells. Albertoni and Lake (2003) obtained negative connectivity values for injector-producer pairs on different sides of a similarly simulated fault. The results of the network model for this system are shown in Figure 3.2. The sealing fault can be inferred either from values of conductance and $k A_{c s}$ (Tables 3.5 and 3.6) or flow between wells (Tables 3.7 and 3.8). Conductance values corresponding to pairs of wells located on each side of the fault are zero. The volume of fluid flowing between them is very small compared to flow between other well-pairs. This shows no conduction between injection wells and production wells that are separated by the fault.

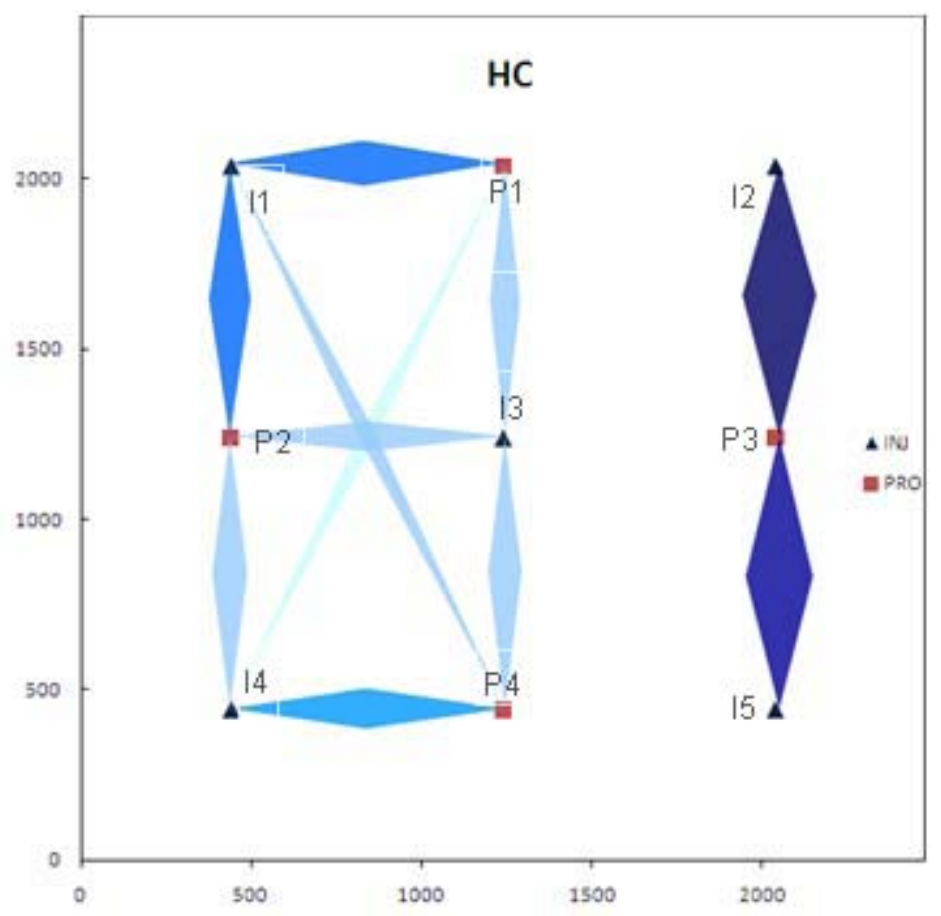

Figure 3.2: Illustration of the conductance and flux shown in Tables 3.5 and 3.7

\subsubsection{Anisotropy}

In this case, permeability in the y direction for each grid cell was set to $\frac{1}{10}$ the permeability in the $x$ direction. Conductance, $k A_{c s}$, flow, and WAF between wells are shown in Tables 3.9, 3.10, 3.11, and 3.12 respectively. Figure 3.3 shows that both conductance and flux are larger in the $x$ direction, indicating that permeability is higher in that specific direction. Unlike the homogenous case, the 
Table 3.5: Presence of barrier: Conductance (d.ft/cp)

\begin{tabular}{ccccc}
\hline & P1 & P2 & P3 & P4 \\
\hline I1 & 0.342 & 0.315 & 0.001 & 0.166 \\
\hline I2 & 0.000 & 0.000 & 0.553 & 0.004 \\
\hline I3 & 0.266 & 0.262 & 0.000 & 0.288 \\
\hline I4 & 0.171 & 0.281 & 0.007 & 0.323 \\
\hline I5 & 0.000 & 0.000 & 0.522 & 0.000 \\
\hline
\end{tabular}

Table 3.6: Presence of barrier: $k A_{c s}$ values $\left(d . f t^{2}\right)$

\begin{tabular}{ccccc}
\hline & P1 & P2 & P3 & P4 \\
\hline I1 & 273 & 251 & 2 & 297 \\
\hline I2 & 0.007 & 0.007 & 442 & 6 \\
\hline I3 & 212 & 209 & 0.008 & 230 \\
\hline I4 & 305 & 224 & 12 & 258 \\
\hline I5 & 0.007 & 0.007 & 417 & 0.05 \\
\hline
\end{tabular}

Table 3.7: Presence of barrier: Cumulative flow between wells $(b b l)$

\begin{tabular}{ccccc}
\hline & P1 & P2 & P3 & P4 \\
\hline I1 & 71894 & 66074 & 295 & 35276 \\
\hline I2 & 2 & 1 & 134295 & 969 \\
\hline I3 & 30360 & 29809 & 1 & 33416 \\
\hline I4 & 17624 & 28848 & 632 & 33913 \\
\hline I5 & 1 & 1 & 103929 & 13
\end{tabular}

Table 3.8: Presence of barrier: Average well allocation factors

\begin{tabular}{ccccc}
\hline & P1 & P2 & P3 & P4 \\
\hline I1 & 0.414 & 0.381 & 0.002 & 0.203 \\
\hline I2 & 0.000 & 0.000 & 0.993 & 0.007 \\
\hline I3 & 0.324 & 0.319 & 0.000 & 0.357 \\
\hline I4 & 0.218 & 0.356 & 0.008 & 0.419 \\
\hline I5 & 0.000 & 0.000 & 1.000 & 0.000 \\
\hline
\end{tabular}


Table 3.9: Anisotropy: Conductance (d.ft/cp)

\begin{tabular}{ccccc}
\hline & P1 & P2 & P3 & P4 \\
\hline I1 & 0.750 & 0.180 & 0.148 & 0.054 \\
\hline I2 & 0.690 & 0.201 & 0.210 & 0.027 \\
\hline I3 & 0.150 & 0.420 & 0.420 & 0.180 \\
\hline I4 & 0.107 & 0.120 & 0.067 & 0.660 \\
\hline I5 & 0.067 & 0.134 & 0.150 & 0.630 \\
\hline
\end{tabular}

Table 3.10: Anisotropy: $k A_{c s}$ values $\left(d . f t^{2}\right)$

\begin{tabular}{ccccc}
\hline & P1 & P2 & P3 & P4 \\
\hline I1 & 600 & 144 & 264 & 96 \\
\hline I2 & 552 & 360 & 168 & 48 \\
\hline I3 & 120 & 336 & 336 & 144 \\
\hline I4 & 192 & 96 & 120 & 528 \\
\hline I5 & 120 & 240 & 120 & 504 \\
\hline
\end{tabular}

effect of injection rate on $k A_{c s}$ values is more tangible. The largest volume of fluid has flowed between pairs I1-P1 and I2-P1.

\subsubsection{Flow paths that act as barriers}

In addition to faults and low permeablity geologic features, a high-flow path may block flow between injector-producer pairs on opposite sides of the flow path. The pressure field might act as a barrier and block the flow from an injector to a producer when there is no physical barrier. To show an example of this case, a high-flow path was introduced by multiplying the y-direction transmissibility of a region of a reservoir by 100. This region connects injector 3 to producers 1 and 4 . To make a stronger flow path, injection in injector 3 is increased, and the BHP constraint of producers 1 and 4 is reduced to direct the injection to those producers. It would be expected that injectorproducer pairs that are on different sides of the high-flow path show weaker connections compared with a homogeneous equal BHP case (Figure 3.1). 
Table 3.11: Anisotropy: Cumulative flow between wells ( $b b l)$

\begin{tabular}{ccccc}
\hline & P1 & P2 & P3 & P4 \\
\hline I1 & 107818 & 30035 & 26170 & 9511 \\
\hline I2 & 74879 & 27383 & 29870 & 3281 \\
\hline I3 & 7722 & 35610 & 37518 & 12887 \\
\hline I4 & 7196 & 10950 & 6960 & 55884 \\
\hline I5 & 5700 & 15367 & 18067 & 64894 \\
\hline
\end{tabular}

Table 3.12: Anisotropy: Average well allocation factors

\begin{tabular}{ccccc}
\hline & P1 & P2 & P3 & P4 \\
\hline I1 & 0.621 & 0.173 & 0.151 & 0.055 \\
\hline I2 & 0.553 & 0.202 & 0.221 & 0.024 \\
\hline I3 & 0.082 & 0.380 & 0.400 & 0.137 \\
\hline I4 & 0.089 & 0.135 & 0.086 & 0.690 \\
\hline I5 & 0.055 & 0.148 & 0.174 & 0.624 \\
\hline
\end{tabular}

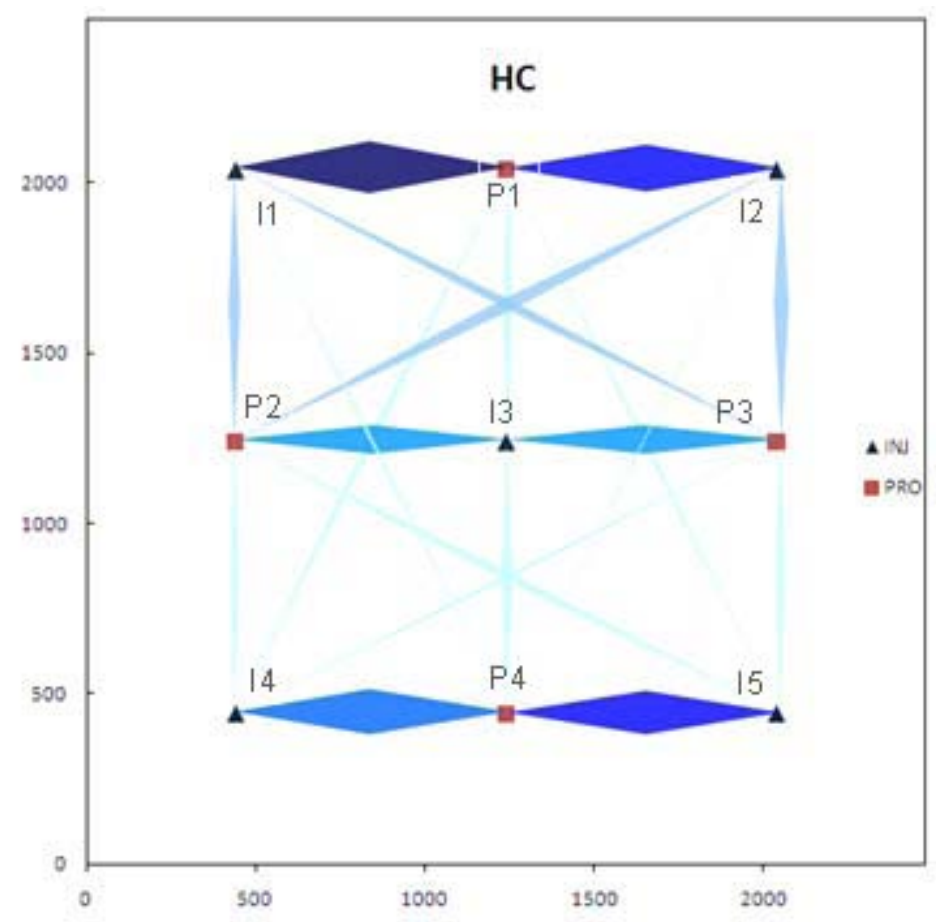

Figure 3.3: Illustration of the conductance and flux shown in Table 3.9 and 3.11 


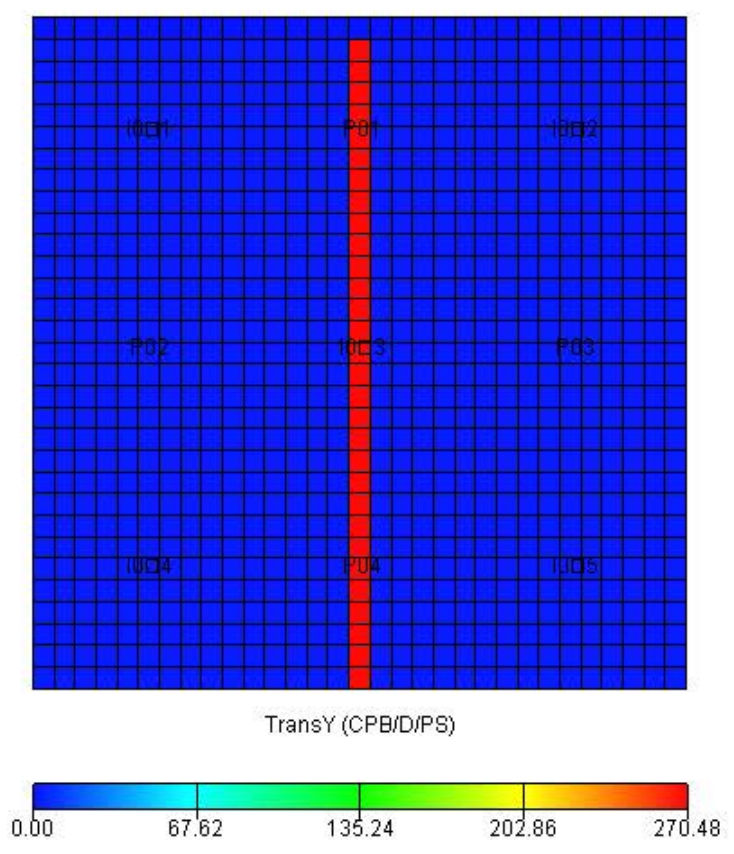

(a) Transmissibility map with a high value in the middle

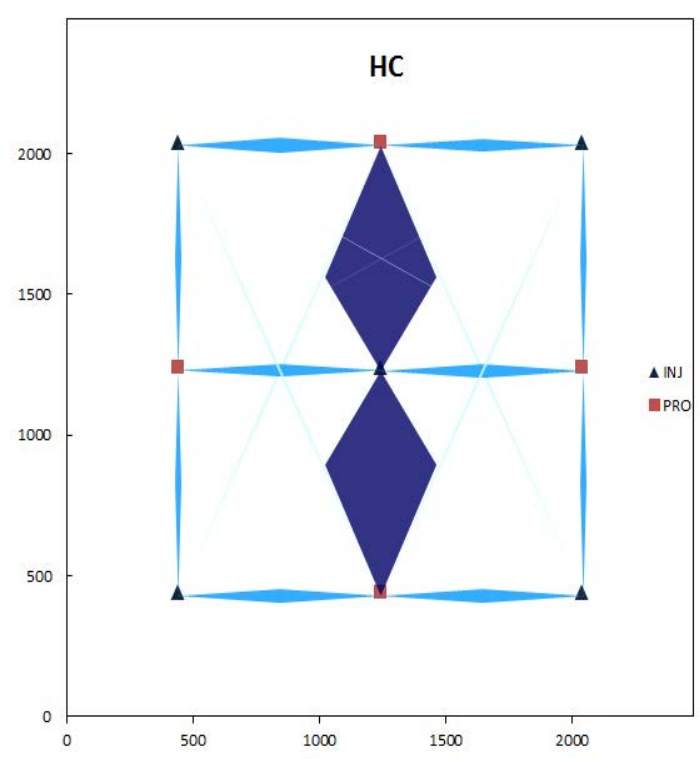

(b) Conductance values

Figure 3.4: Flow paths that act as barriers.

To show this, conductance values are plotted next to a transmissibility map in Figure 3.4 a and b. High conductance values between injector 3 and producers 1 and 4 reflect the high y-direction transmissibility region, and low value for wells on different sides of the region means they do not have connections. Well allocation factors also indicate that the effects of injector 1 and 4 on producer 3 and injectors 2 and 5 on producer 2 are dramatically reduced. While in the base case, 13 percent of injection in injector 2 goes to producer 2 , in this case the effect is reduced to 2 percent. This shows that the network model approach can see the effect of pressure barriers in addition to permeability barriers.

\subsubsection{Conductance and permeability}

In Eq. 2.2, it was shown that conductance is directly proportional to permeability. Here, we are trying to show how results from the network model reflect changes in the system's average permeability in spite of the nonuinque nature of the process. Conductance values obtained from five homogeneous cases with $20 \mathrm{md}, 30 \mathrm{md}, 40 \mathrm{md}$, $60 \mathrm{md}$, and $80 \mathrm{md}$ were compared to check if 
changes in conductivity values are proportional to the permeability change in the synthetic models. To make such a comparison, the distance element of conductance is removed, which gives $k A_{c s}$ (product of permeability and cross-section area open to flow). Then $k A_{c s}$ values are averaged for each case and compared with avarage permeability of the synthetic models. Figure 3.5 shows that avarage $k A_{c s}$ increases linearly with average reservoir permeability. This shows that although an inverse problem is being solved and the result is not unique $k A_{c s}$ values and as a result conductance values reflect the system's average permeability.

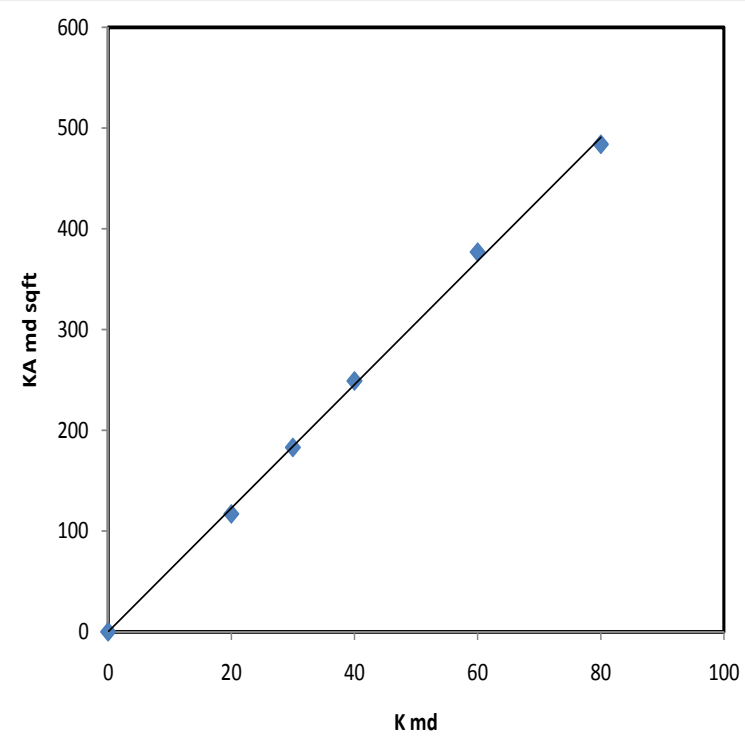

Figure 3.5: Change in $k A_{c s}$ as permeability changes in sythetic model

Figure 3.6 is another example to show proportionality of the obtained conductance values to the field average pressure. The field has two permeability regions and permeability in one half of the reservoir is twice that of the other half (Figure 3.6 a). Producers 1 and 2 are in the low-permeability region, and producers 3 and 4 are in the high-permeability region. Conductance values that relate injector 3 to producers 1 and 2 are half the conductance values that relate this injector to producers 3 and 4 . The result confirms the doubled average permeability between injector 3 and producers 3 and 4 by maintaining this proportionality. 


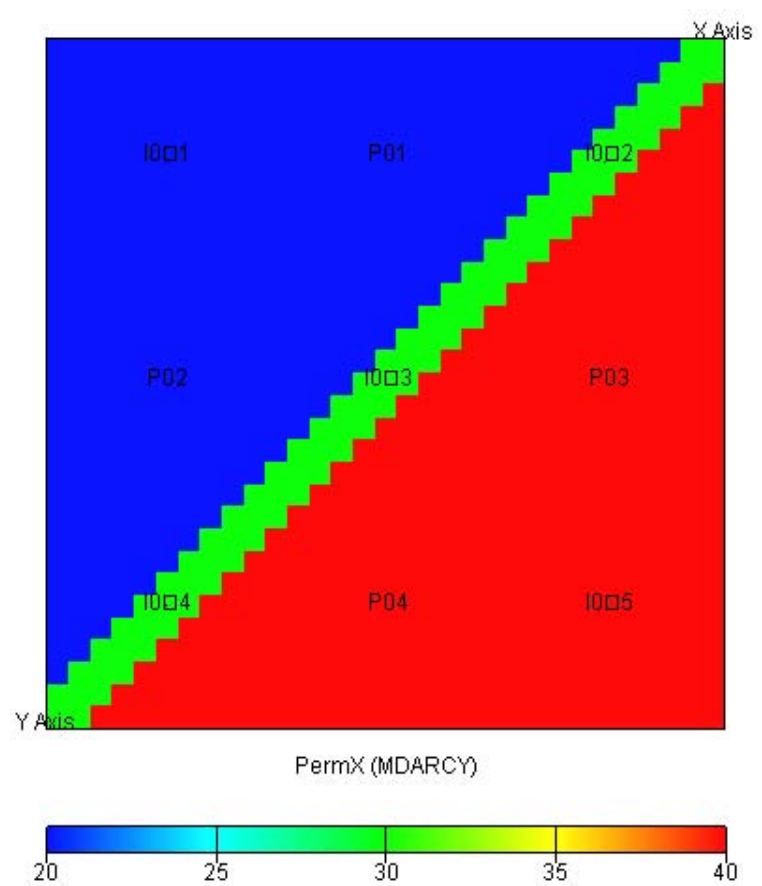

(a) Permeability distribution in the domain

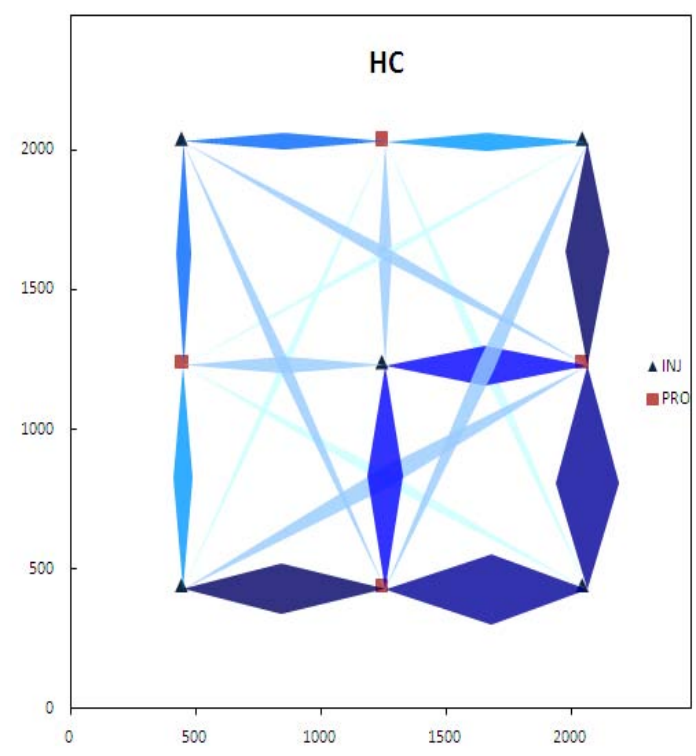

(b) Conductance

Figure 3.6: Two permeability regions in the domain.

\subsubsection{Complex geological features}

Complex geologic features are very common in reservoir studies. Because of these features, streamlines may travel different paths that are not the straight line that connects injection to production wells. Therefore conductance values should be interpreted with more consideration. In this example, a channel structure with permeabiliy that is 10 times larger than the rest of the reservoir model connects injection well 2 to production well 2 . Since production well 2 is placed in a highly permeable region, it has higher productive capabilities. Figure 3.7 is plotted using the FrontSim ${ }^{\circledR}$ software with simulation parameters set up as discussed in section 3.3.1 and using the permeability distribution of Figure 3.8. As shown in Figure 3.7, more streamlines end at production well 2, and nonadjacent wells have considerable contribution. Figure 3.9 shows the network model result for this case. Although one cannot infer the shape of the channel from the conductance values, the existance of some anomaly around production well 2 is noticable. In comparison to Figure 3.1, all the injection wells have much stronger influence on production well 2 than in the homogeneous case. All of the other producers are receiving significently less support, which indicates 
the heterogeneity is more widespread that just in the region around producer 2 and is a "regional trend".

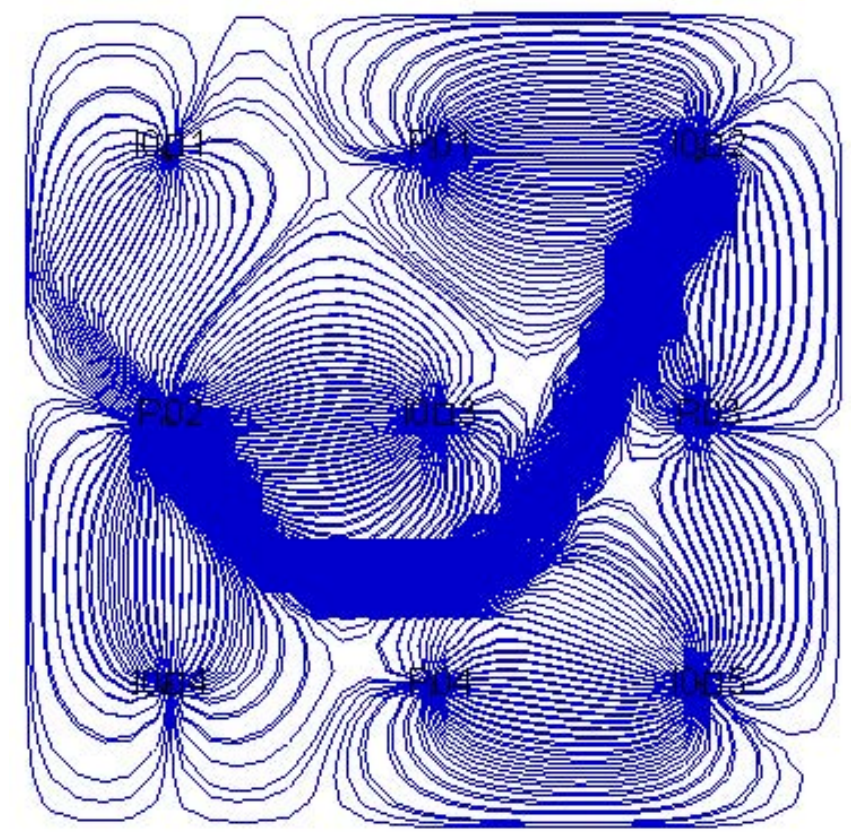

Figure 3.7: Distribution of streamlines in the domain

\subsection{Prediction and comparison}

One of the challenges of simplified methods like correlation-based methods is their inability to handle certain cases and scenarios. Shutting-in injectors and producers, changing flow pattern and non-fluctuating injection are examples of cases that challenge available methods. In this section, the network model was used to see how it performs in each of these cases.

\subsubsection{Closed Well}

Shutting in a producer or adding a new well to the system is fairly routine in waterflooding. Coefficients calculated by currently available methods are only valid when a fixed number of producers and injectors is being studied. Once a well is added or removed from the system, coefficents are no longer valid and need to be redetermined. This limits the current methods to cases where there are sufficient continuous data from the active wells. In the situation where all wells are producing, 


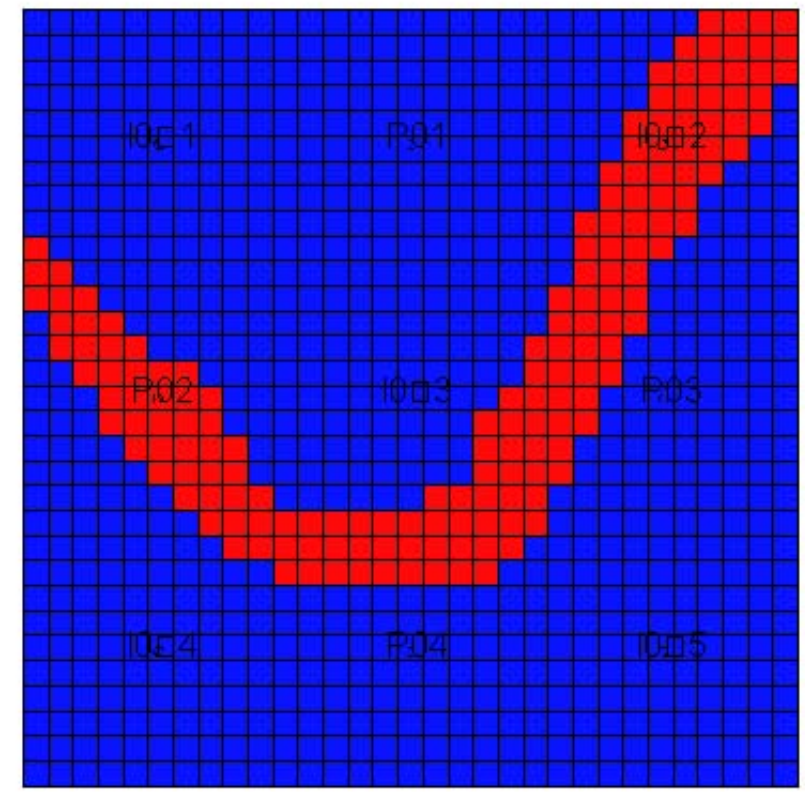

PermX (MDARCY)

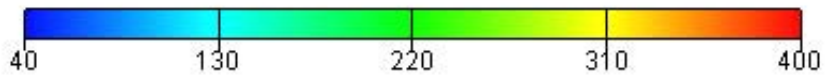

Figure 3.8: Permeability distribution in the domain

all nodes have a sink representing the producing well. Once a production well is shut in, the sink is removed but the node is still interacting with other nodes through the bonds. This helps to study broader time intervals and includes those times when wells are shut in.

This methodology can be shown using the homogeneous case of example 1. Production well 1 was added to the system after 30 months of field production and was produced for the next 20 months. Then production data during the first 50 months were used to predict production for the next 50 months. Taking advantage of the whole production period, the network model predicts production better than other methods.

In the current method, the production data during 50 timesteps including the first 30 timesteps when 3 producers were active are studied to predict the next 50 timesteps when 4 producers are active. The CM and RM method are unable to use the first 30 timesteps and are limited to the 20 timesteps when all 4 producers were active. Therefore, this method gives the opportunity to study 


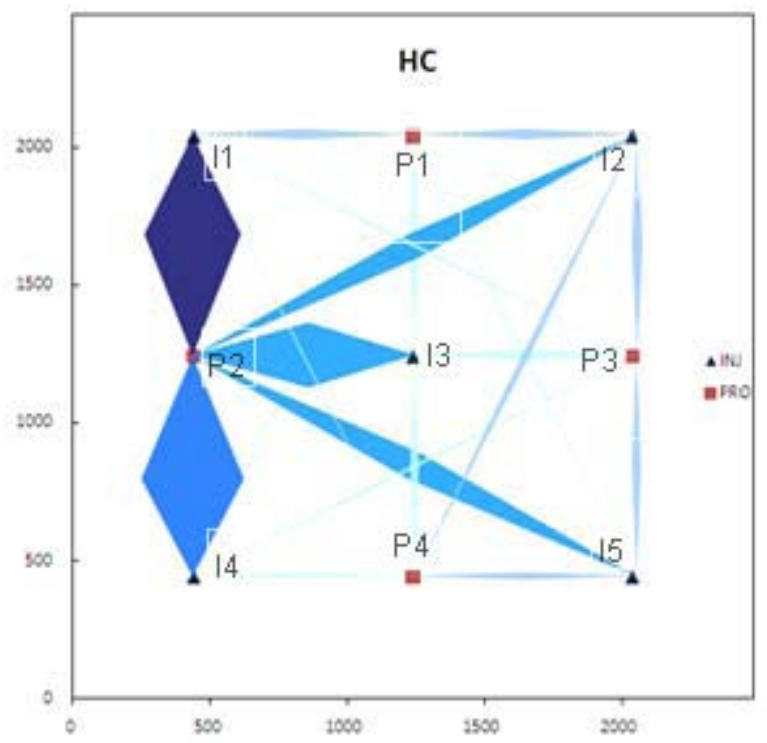

Figure 3.9: High permeable channel: Conductance values

a broader time inerval, which results in a more robust prediction. Table 3.13 shows the correlation coefficient (R-Squared) values for production prediction for the 50 time-step interval.

Table 3.13: Yield R-Squared for production prediction when one well is added to the system

\begin{tabular}{lcccc}
\hline & P1 & P2 & P3 & P4 \\
\hline RM & 0.893 & 0.924 & 0.965 & 0.957 \\
\hline CM & 0.996 & 0.990 & 0.956 & 0.979 \\
\hline Network & 0.999 & 0.998 & 0.993 & 0.998 \\
\hline
\end{tabular}

\subsubsection{Injection fluctuation}

In the network model, the number of equations is equal to the number of observations. If injection rate doesn't fluctuate, the equations won't be independent. A case was tested with all injecting wells having equal constant rates. For this case, the number of observations is less than the number of variables, and therefore it is an underdetermined problem, and similar to correlation-based approaches, there is no consistant solution to this problem. 
Conductance values are plotted in the Figure 3.10, which doesn't agree with previous results. What is shown in Figure 3.10 a also depends on starting point in the optimization routine because of the unstable nature of the system.

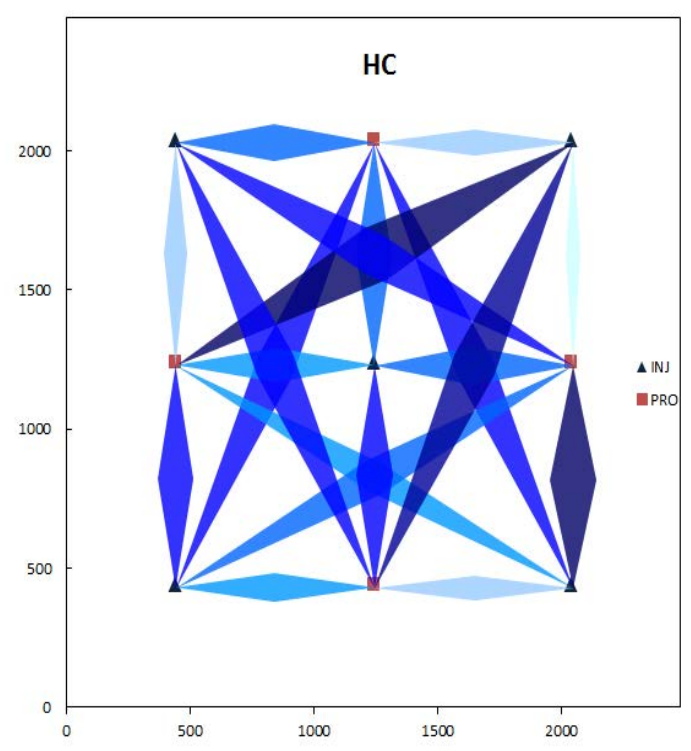

(a) Constant injection rate

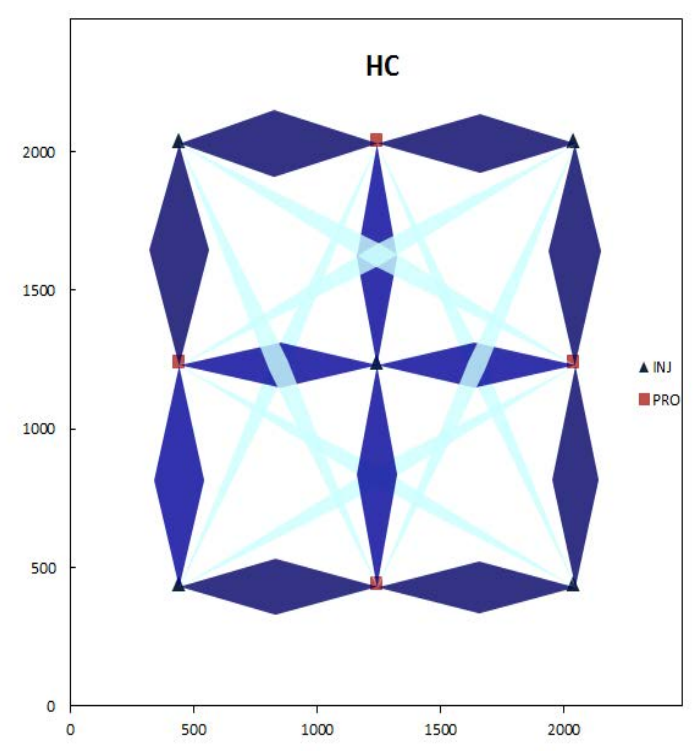

(b) Injection with one fluctuation

Figure 3.10: Injection fluctuation.

If one injection rate changes slightly, this causes changes in the production data in at least one timestep (the effect of this fluctuation may continue in subsequent timesteps), the number of observations increase, and the problem changes from an underdetermined case to an overdetermined one and a reliable solution results $(3.10 \mathrm{~b})$. An increase in the number of fluctuations will assure an overdetermined problem.

\subsubsection{Changing flow pattern}

Once conductance values are known, one can predict perfomance of the system under different injection senarios. To test the prediction potential of the method, the homogenous example system of section 3.3.1 was chosen. Injection rates from the first 65 time steps of this example were used to determine the conductance values. Then these conductance values were used to predict production for the next 35 time steps. In the next 35 timesteps, the injection rates in the finite-difference 
Table 3.14: Yield R-squared values of production match for RM, CM and Network model

\begin{tabular}{lcccc}
\hline & P1 & P2 & P3 & P4 \\
\hline Albertoni & 0.862 & 0.933 & 0.906 & 0.954 \\
\hline Yousef & 0.983 & 0.996 & 0.967 & 0.990 \\
\hline Network & 0.997 & 0.999 & 0.999 & 0.997 \\
\hline
\end{tabular}

simulation used to generate the problem were manipulated to change the direction of the pressure gradient and the direction of flow in the model.

During the first 65 time steps, the average injection rate at injector 4, which is at the lower left corner, was less than the average injection rate in injector 2 , which is at the upper right corner. This results in a lower pressure region south of injector 1, which is at the upper left corner, and more flow from injector 1 towards the bottom of the system. A change in flow direction was attempted by increasing the average injection rate at injector 4 and decreasing the average injection rate at injector 2. This should cause a lower pressure region to the right of injector 1 and more flow from injector 1 toward the right side of the model. The goal was to find out how good the network model approach can predict production in the next 35 timesteps by comparing the result with the RM and $\mathrm{CM}$ approaches. The R-squared values of the matches of production rate using the RM, the CM, and the Network model approach for four production wells are presented in Table 3.14.

In the network model, the direction of flow is determnined by the pressure gradient, which leads to a good match, as is shown in Table 3.14. The RM and CM results also show satisfactory but slightly lower $R^{2}$ values for their predictions.

\subsection{Including additional data}

The output from the method can be improved significently by using better quality data and adding more data to the system. For example, more accurate values for thickness and boundary distance help to obtain more realistic results. The addition of fault positions and more accurate well indices for producing wells also improve the result. Including fault positions in the process of assigning volume to a well results in estimation of more realistic node volumes. Including more accurate 
well indices helps to infer communication between the node and the well and improves the result. For the homogeneous case, using well index values from the simulation software used to generate the rate profile reduces the difference between observed and model production by $30 \%$ over the result obtained using very large well index values. From this point, conductance values are compared with conductance values of Table 3.15, assuming production well indices are known. The new conductance values are twice those found with the large well indices presented in Table 3.1. Well allocation factors (Table 3.16) show that injectors have a higher influence on adjacent wells compared to a case when large well index values were used. In the next section, the importance of input parameters and the effect of using incorrect parameters on conductance values will be studied.

Table 3.15: Conductance values for a model with known WI $(d . f t / c p)$

\begin{tabular}{ccccc}
\hline & P1 & P2 & P3 & P4 \\
\hline I1 & 0.771 & 0.773 & 0.244 & 0.242 \\
\hline I2 & 0.815 & 0.259 & 0.814 & 0.260 \\
\hline I3 & 0.577 & 0.582 & 0.584 & 0.589 \\
\hline I4 & 0.284 & 0.877 & 0.284 & 0.875 \\
\hline I5 & 0.227 & 0.231 & 0.724 & 0.732 \\
\hline
\end{tabular}

Table 3.16: Well allocation factors for a model with known WI

\begin{tabular}{ccccc}
\hline & P1 & P2 & P3 & P4 \\
\hline I1 & 0.371 & 0.381 & 0.123 & 0.125 \\
\hline I2 & 0.364 & 0.122 & 0.386 & 0.129 \\
\hline I3 & 0.229 & 0.245 & 0.255 & 0.271 \\
\hline I4 & 0.113 & 0.363 & 0.124 & 0.400 \\
\hline I5 & 0.109 & 0.117 & 0.377 & 0.397 \\
\hline
\end{tabular}




\subsubsection{Well index and bottomhole pressure}

Bottomhole pressure (BHP) and well index (WI) are two important sources of uncertainty in the network model, which the latter is related to near-well permeability. Low WI and higher BHP values reduce and high WI and low BHP increase the amount of fluid flow to producers. Both parameters influence the connectivity value calculation and therefore near-well permeability interpretation. To investigate the effect of each parameter on conductance and well allocation factor, four cases were studied.

In all cases, the same simulated injection scenario was applied. In the first and second cases, the permeability field is homogeneous, but a different BHP value is assigned to one of the producers. All producers are producing under a 500 psi BHP constraint, except producer 2, which is producing with a 600 psi BHP constraint. The difference is that in case one, the BHP difference is considered known in the network model, but in case two it is assumed that all producers are producing at the 500 psi BHP. In cases three and four the BHP values are held constant at 500 psi, but the permeability is lowered to $20 \mathrm{md}$ around well 2, as shown in Figure 3.11. Again, the difference in the cases is that knowledge of the permeability variation is reflected in the well index used for producer 2 in case three, but in case four, equal values of the WI for all producers are used.

For case one, well allocation factors are smaller for producer 2 (Table $3.17 \mathrm{a}$ and b). Conductance values do not show heterogeneity, which is a good indication of a homogenous field and different buttomhole pressure (Figure $3.12 \mathrm{a}$ and b). For a homogeneous case, if an incorrect BHP is used (case two), depending on whether a higher or a lower pressure is used, larger or smaller conductance values are obtained for that specific well (Table $3.18 \mathrm{a}$ and b). Smaller or larger conductance values are the response of the model to compensate for the higher or lower pressure difference between injector-producer pairs. For example in case two, the BHP that is assigned to producer 2 is less than the actual BHP of the well (Figure $3.13 \mathrm{a}$ and b). The obtained conductance values for producer 2 are smaller compared to conductance values in case one to reduce the effect of the incorrect higher pressure difference between well and the block. 
Table 3.17: Different BHP at producer 2.

(a) Conductance $(d . f t / c p)$

\begin{tabular}{ccccc}
\hline & P1 & P2 & P3 & P4 \\
\hline I1 & 0.792 & 0.789 & 0.242 & 0.239 \\
\hline I2 & 0.857 & 0.253 & 0.840 & 0.267 \\
\hline I3 & 0.658 & 0.650 & 0.659 & 0.671 \\
\hline I4 & 0.325 & 0.973 & 0.315 & 1.002 \\
\hline I5 & 0.219 & 0.218 & 0.718 & 0.731 \\
\hline
\end{tabular}

(b) Average well allocation factors

\begin{tabular}{ccccc}
\hline & P1 & P2 & P3 & P4 \\
\hline I1 & 0.386 & 0.366 & 0.124 & 0.124 \\
\hline I2 & 0.372 & 0.106 & 0.394 & 0.129 \\
\hline I3 & 0.239 & 0.208 & 0.268 & 0.285 \\
\hline I4 & 0.120 & 0.311 & 0.131 & 0.437 \\
\hline I5 & 0.108 & 0.099 & 0.386 & 0.406 \\
\hline
\end{tabular}

In case three, the decreased permeability value for producer 2 is reflected in the conductance values of Table 3.19 a. Well allocation factors in Table $3.19 \mathrm{~b}$ also confirm this difference. In case four, where a higher WI is used for producer 2, smaller values of conductance are obtained to compensate for the incorrect higher communication between the well and the node (Table $3.20 \mathrm{a}$ and $b)$.

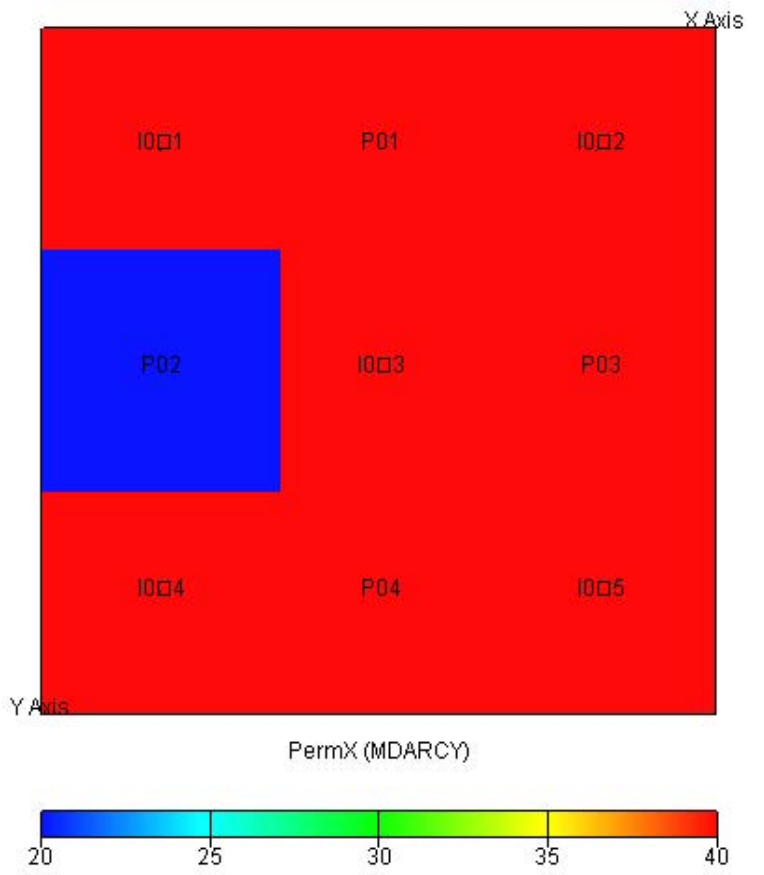

Figure 3.11: Permeability distribution in the domain 


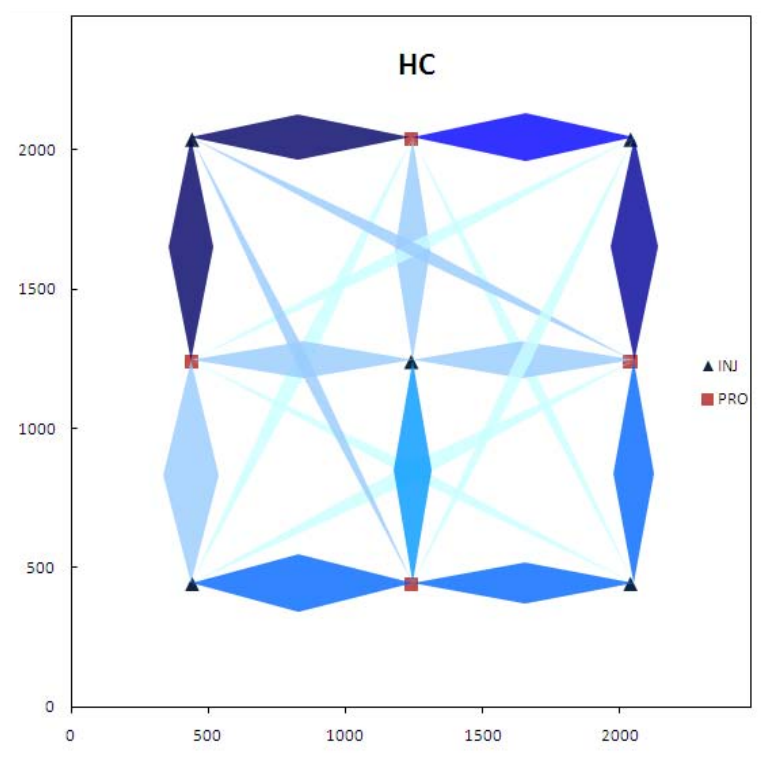

(a) Different BHP

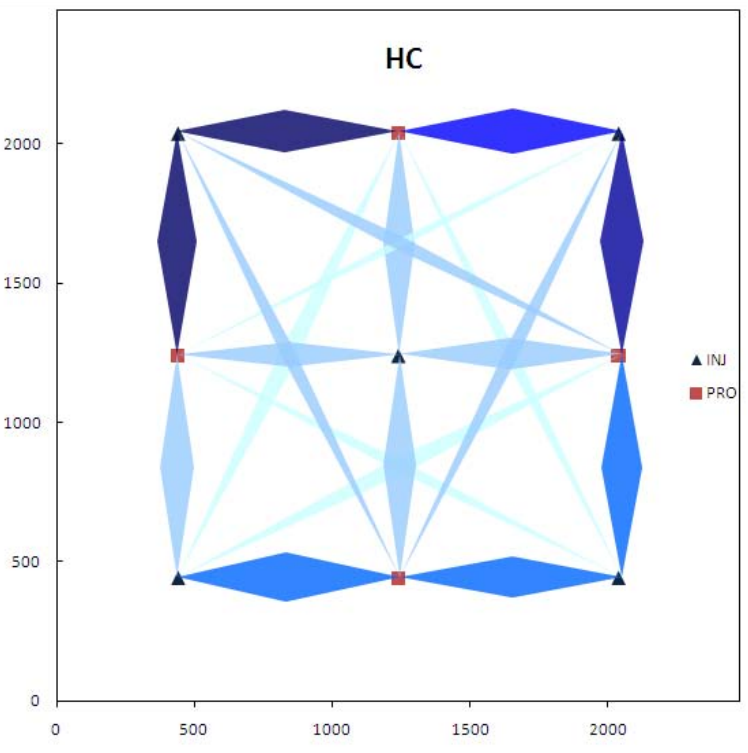

(b) Wrong BHP

Figure 3.12: Effect of BHP on conductance values.

Table 3.18: Incorrect BHP at producer 2.

(a) Conductance $(d . f t / c p)$

\begin{tabular}{ccccc}
\hline & P1 & P2 & P3 & P4 \\
\hline I1 & 0.779 & 0.708 & 0.255 & 0.257 \\
\hline I2 & 0.785 & 0.152 & 0.769 & 0.269 \\
\hline I3 & 0.568 & 0.448 & 0.563 & 0.580 \\
\hline I4 & 0.342 & 0.594 & 0.320 & 0.889 \\
\hline I5 & 0.229 & 0.209 & 0.713 & 0.727 \\
\hline
\end{tabular}

(b) Average well allocation factors

\begin{tabular}{ccccc}
\hline & P1 & P2 & P3 & P4 \\
\hline I1 & 0.372 & 0.370 & 0.128 & 0.131 \\
\hline I2 & 0.379 & 0.083 & 0.395 & 0.143 \\
\hline I3 & 0.237 & 0.226 & 0.259 & 0.278 \\
\hline I4 & 0.140 & 0.296 & 0.144 & 0.420 \\
\hline I5 & 0.111 & 0.119 & 0.375 & 0.395 \\
\hline
\end{tabular}

This comparison shows that using incorrect values of BHP and WI still provide correct values of WAFs. However, the error in estimation of these input parameters for a producer results in similar error in the obtained conductance values for that well.

\subsubsection{Node volume and compressibility}

Like BHP and WI, node volumes and fluid compressibility influence the calculated parameters. In this section, two cases were studied. In the first case, node volumes are underestimated by $30 \%$, and in the second, the assumed compressibility in the model is $30 \%$ lower that actual reservoir 


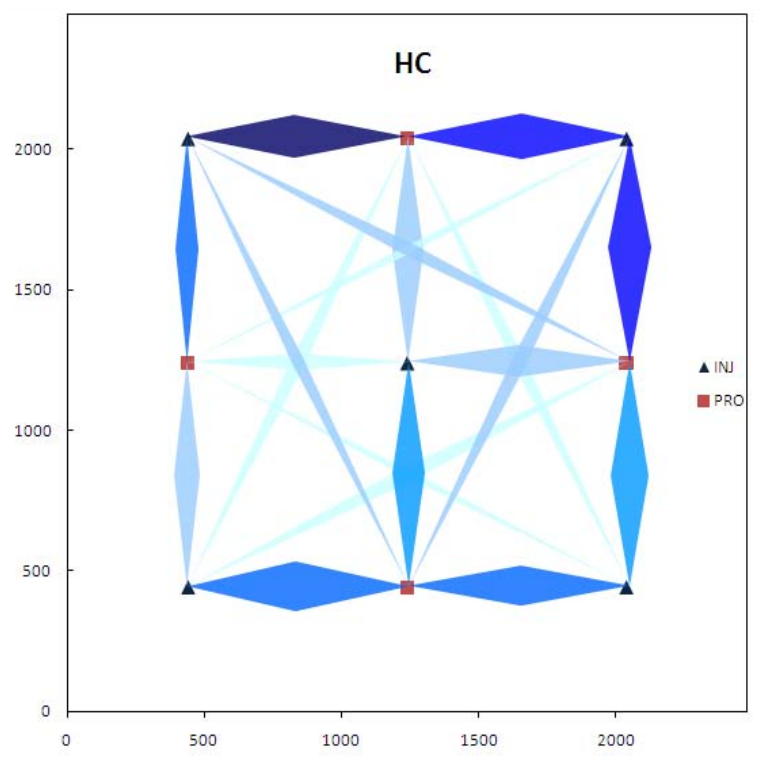

(a) Different WI

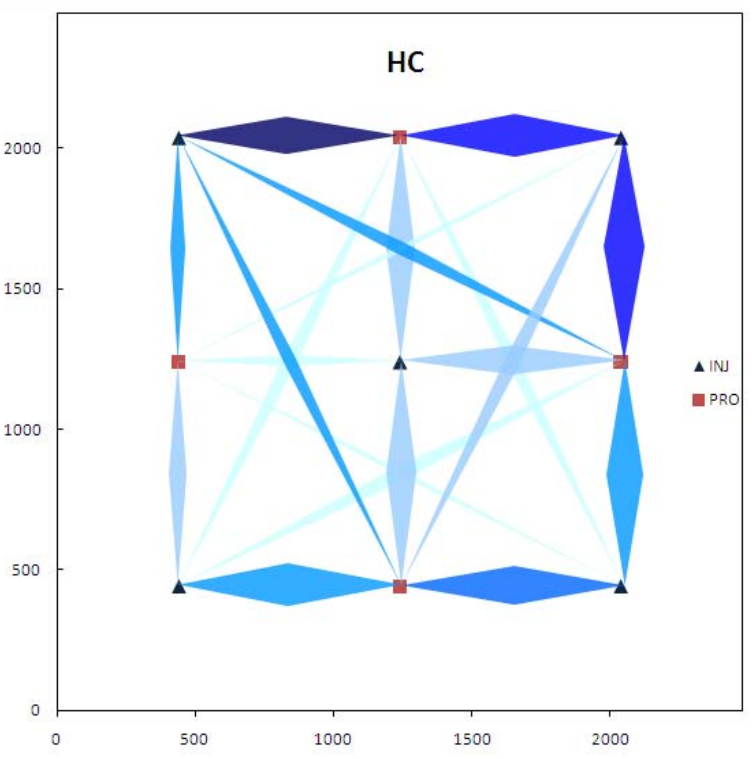

(b) Wrong WI

Figure 3.13: Effect of WI on conductance values.

Table 3.19: Different WI at producer 2.

(a) Conductance (d.ft/cp)

\begin{tabular}{|c|c|c|c|c|c|c|c|c|c|}
\hline & $\mathrm{P} 1$ & $\mathrm{P} 2$ & P3 & P4 & & $\mathrm{P} 1$ & $\mathrm{P} 2$ & P3 & P4 \\
\hline I1 & 0.758 & 0.405 & 0.241 & 0.224 & I1 & 0.457 & 0.245 & 0.154 & 0.144 \\
\hline $\mathrm{I} 2$ & 0.816 & 0.151 & 0.787 & 0.274 & $\mathrm{I} 2$ & 0.381 & 0.073 & 0.402 & 0.144 \\
\hline I3 & 0.570 & 0.301 & 0.557 & 0.584 & I3 & 0.260 & 0.138 & 0.290 & 0.311 \\
\hline I 4 & 0.267 & 0.458 & 0.286 & 0.866 & I4 & 0.130 & 0.222 & 0.158 & 0.490 \\
\hline I5 & 0.267 & 0.458 & 0.286 & 0.866 & I5 & 0.120 & 0.068 & 0.393 & 0.420 \\
\hline
\end{tabular}

compressibility. The results are shown in Table 3.21 and Table 3.22 compared with conductance values and WAFs presented in Table 3.15 and Table 3.16. Underestimating reservoir volume and compressibility results in lower computed conductance values. Both situations speed up the movement of the pulse generated by injection fluctuations to the producers, and computed conductance values are lowered to delay this process. WAFs also show less adjacent injector-producer support.

The results show that conductance values and WAFs are sensitive to node volume and compressibility, and misestimating these inputs influence the set of obtained parameters. 
Table 3.20: Incorrect WI at producer 2.

(a) Conductance $(d . f t / c p)$

\begin{tabular}{|c|c|c|c|c|c|c|c|c|c|}
\hline & $\mathrm{P} 1$ & $\mathrm{P} 2$ & P3 & $\mathrm{P} 4$ & & $\mathrm{P} 1$ & $\mathrm{P} 2$ & P3 & $\mathrm{P} 4$ \\
\hline I1 & 0.684 & 0.263 & 0.237 & 0.229 & I1 & 0.456 & 0.215 & 0.167 & 0.162 \\
\hline $\mathrm{I} 2$ & 0.770 & 0.110 & 0.741 & 0.274 & I2 & 0.377 & 0.076 & 0.396 & 0.151 \\
\hline I3 & 0.521 & 0.201 & 0.506 & 0.534 & I3 & 0.257 & 0.157 & 0.282 & 0.304 \\
\hline I4 & 0.266 & 0.295 & 0.275 & 0.768 & I4 & 0.138 & 0.244 & 0.161 & 0.457 \\
\hline I5 & 0.236 & 0.097 & 0.652 & 0.686 & I5 & 0.125 & 0.078 & 0.385 & 0.412 \\
\hline
\end{tabular}

Table 3.21: Incorrect node volume.

(a) Conductance $(d . f t / c p)$

\begin{tabular}{|c|c|c|c|c|c|c|c|c|c|}
\hline & P1 & P2 & P3 & P4 & & P1 & P2 & P3 & P4 \\
\hline I1 & 0.360 & 0.361 & 0.148 & 0.148 & I1 & 0.350 & 0.354 & 0.147 & 0.149 \\
\hline I2 & 0.382 & 0.158 & 0.382 & 0.158 & I2 & 0.346 & 0.147 & 0.356 & 0.151 \\
\hline I3 & 0.271 & 0.274 & 0.275 & 0.278 & I3 & 0.238 & 0.247 & 0.253 & 0.262 \\
\hline I4 & 0.174 & 0.408 & 0.174 & 0.407 & I4 & 0.143 & 0.345 & 0.150 & 0.362 \\
\hline I5 & 0.139 & 0.143 & 0.341 & 0.345 & I5 & 0.139 & 0.145 & 0.352 & 0.364 \\
\hline
\end{tabular}

(b) Average well allocation factors

\subsection{Pressure and saturation dependent properties}

Average reservoir pressure in a waterflood is a function of the total injection and production. It increases or decreases in nonlinear ways. Differences in properties of water and reservoir fluids cause this nonlinearity. Viscosity and compressibility differences and the shapes of relative permeability curves are factors that influence the average reservoir pressure. These factors may increase or reduce the resistance to flow between injectors and producers. High resistance to flow increases and low resistance to flow decreases the average reservoir pressure.

As noted previously, in order to simplify the problem, it was assumed that fluids with identical properties are injected and produced in the previous examples. The compressibility and viscosity of both fluids were equal ( $1 c p$ viscosity and $5 \times 10^{-6} p s i^{-1}$ compressibility), and linear relative 
Table 3.22: Incorrect compressibility.

(a) Conductance $(d . f t / c p)$

\begin{tabular}{|c|c|c|c|c|c|c|c|c|c|}
\hline & P1 & P2 & P3 & P4 & & P1 & P2 & P3 & P4 \\
\hline I1 & 0.360 & 0.361 & 0.148 & 0.148 & I1 & 0.350 & 0.354 & 0.147 & 0.149 \\
\hline I2 & 0.382 & 0.158 & 0.382 & 0.158 & I2 & 0.346 & 0.147 & 0.356 & 0.151 \\
\hline I3 & 0.272 & 0.275 & 0.276 & 0.279 & I3 & 0.238 & 0.247 & 0.253 & 0.262 \\
\hline I4 & 0.174 & 0.408 & 0.174 & 0.407 & I4 & 0.143 & 0.345 & 0.150 & 0.362 \\
\hline I5 & 0.139 & 0.143 & 0.341 & 0.345 & I5 & 0.139 & 0.145 & 0.352 & 0.364 \\
\hline
\end{tabular}

permeability curves were implemented to model the displacement. In a linear relative permeability curve, relative permeability is a linear function of saturation $\left(k_{r w}=S_{w}, k_{r n w}=S_{n w}\right)$.

Once these conditions hold, one can assume that resistance to flow is constant, and average reservoir pressure is only a function of total fluid throughput and withdrawal. But in practice, saturationdependent properties like relative permeability are changing as the saturation distribution changes. Relative permeabilty alteration influences effective permeabilty, injectivity, and productivity of wells. This changes resistance to flow, and as a result average reservoir pressure. When resistance to flow is constantly changing, one set of conductance values should not be expected to model the whole study period. Changing average reservoir pressure also will change the compressibility and viscosity, which are assumed to be constant in the network model.

The effects of a realistic relative permeability curve on conductance value determination is studied by using the relative permeability relationship from model 2 of the SPE comparative solution project (SPECSP) by Christie and Blunt (2001). Other properties are held constant. Figure 3.14 shows changes in average reservoir pressure and watercut with total injection and time for both linear and SPECSP relative permeability curves. In the linear relative permeability case, the average reservoir pressure is proportional to the total injection rate. Although the SPECSP case follows the same pressure trend at the beginning of the time period, it increases gradually as water saturation increases. This increase accelerates at breakthrough, but the reservoir pressure curve moves closer to the linear curve average reservoir pressure at the end of study period. The reason behind 
this difference is that the sum of the relative permeability of water and oil is less than 1 (the sum of the effective permeability of the fluids is less than when using linear relative permeability curves). This causes more resistance to flow as compared to the linear relative permeability case. Identical injection histories were used, so the higher resistance to flow in the non-linear system increases the average reservoir pressure.

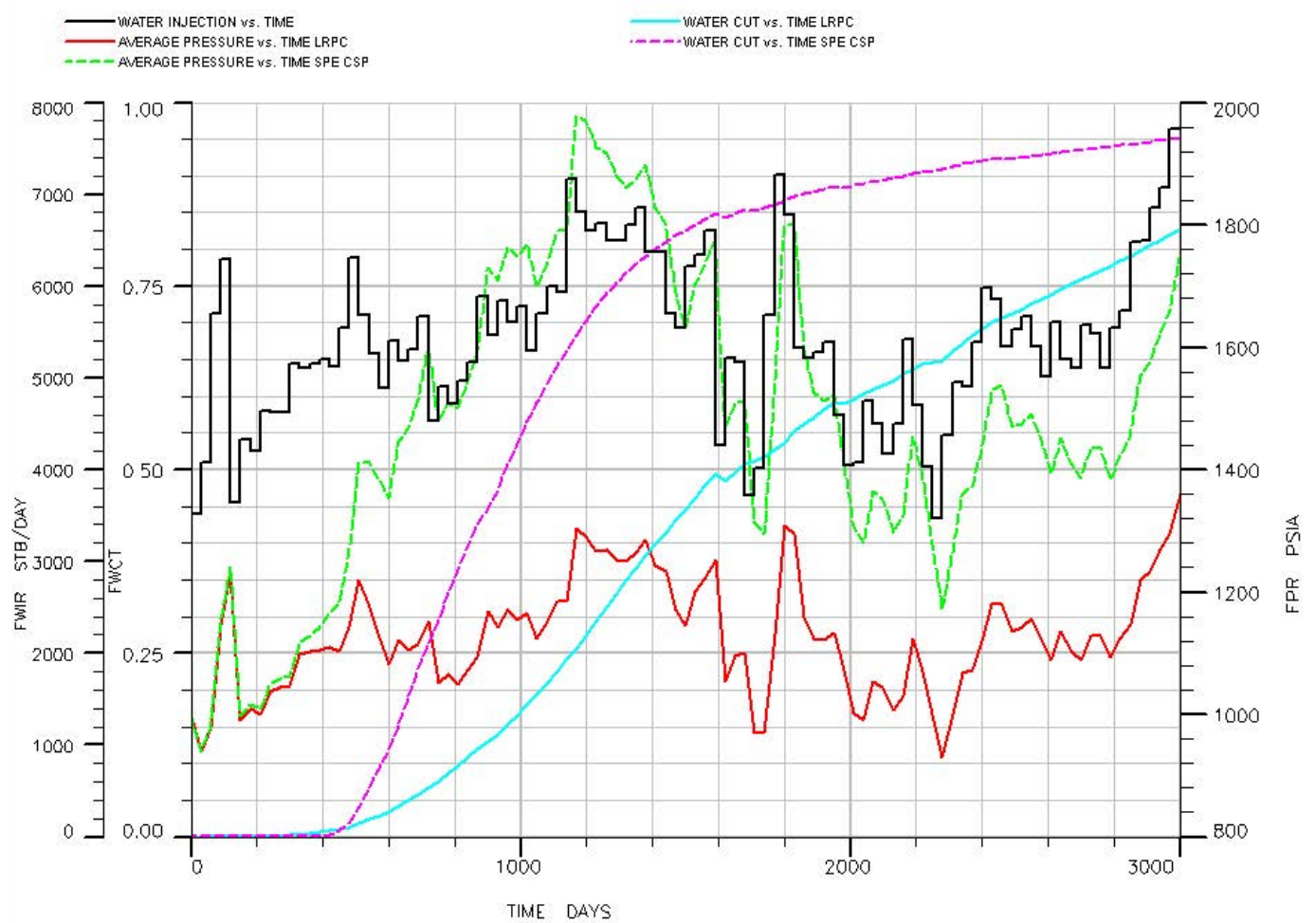

Figure 3.14: Pressure difference for different relative permeability table.

Reduction in effective permeability should decrease the conductance values. To test this hypothesis, a case with linear relative permeability curve is compared with a case that uses the SPECSP relative permeability values. All other input parameters are the same as the ones that are used in the example presented in section 3.5.

Comparing values in Table 3.23 with those in Table 3.15 shows that there was a $100 \%$ reduction in conductance due to the reduction of $k$ to account for relative permeability effects. This decline varies, depending on the shape of the relative permeability curves, since $k$ is a function of satura- 
tion, and saturation changes through time. What is calculated in the network model as conductance is then an average effective permeability during the study period.

Table 3.23: Conductance for single phase homogenous reservoir with SPECSP relative permeability $(d . f t / c p)$

\begin{tabular}{ccccc}
\hline & P1 & P2 & P3 & P4 \\
\hline I1 & 0.323 & 0.304 & 0.132 & 0.080 \\
\hline I2 & 0.293 & 0.144 & 0.324 & 0.184 \\
\hline I3 & 0.241 & 0.226 & 0.237 & 0.250 \\
\hline I4 & 0.178 & 0.328 & 0.171 & 0.358 \\
\hline I5 & 0.100 & 0.107 & 0.248 & 0.286 \\
\hline
\end{tabular}

Injecting a different-viscosity fluid was also studied by using fluid viscosities from model 2 of the SPECSP (Christie and Blunt 2001) while other properties were held constant. Water viscosity is $0.3 c p$, and oil viscosity is pressure dependent and varies between $2.85 \mathrm{cp}$ and $3 \mathrm{cp}$. Figure 3.15 shows changes in average reservoir pressure and watercut with total injection and time for both equal viscosity and the SPECSP viscosity values. Figure 3.15 shows that average reservoir pressure is higher for the SPECSP viscosity values compared to equal values $(1 c p)$ at the start of injection. This is because higher oil viscosity causes more resistance to flow. But as water injection continues, the average pressure drops rapidly and falls below the average reservoir pressure of the equal-viscosity case, which is because of the lower water viscosity.

Conductance values obtained from a simulation model that uses the SPECSP viscosity values are shown in Table 3.24. In this table all injector connections except injector 5 show high conductance values. Water breakthrough at producers creates a low resistance conduit for fluid flow between well pairs due to the low viscosity of the water. That results in higher conductance values compared to values in Table 3.15. For injector 5, which has lower average injection rate, breakthrough happens later in the study period, and results in a lower conductance value for that injector.

Relative permeability and viscosity are both part of the conductance term, and variations of these two parameters change the computed conductance value. Relative permeability and viscosity are 


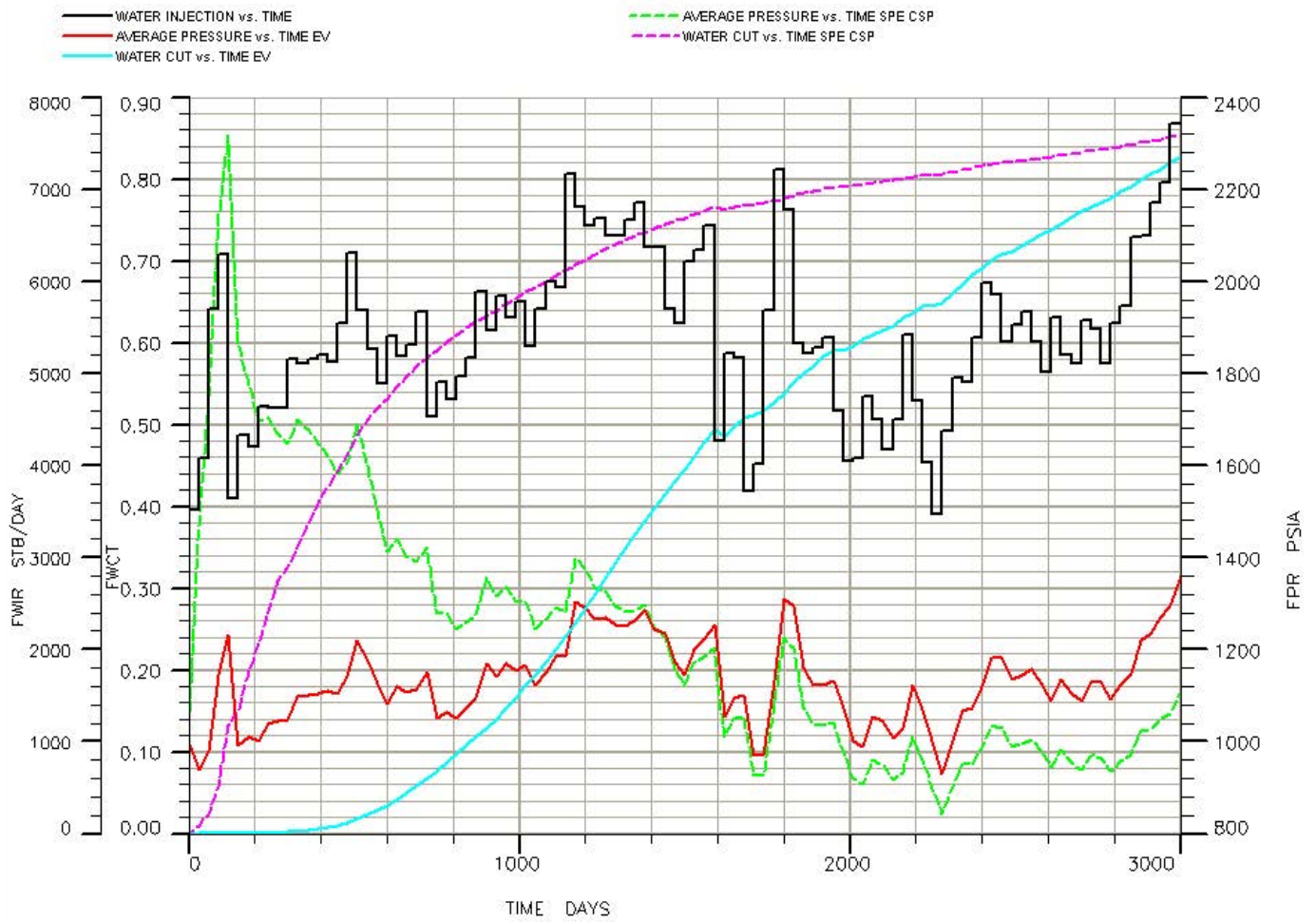

Figure 3.15: Pressure difference for different oil viscosity.

functions of phase saturation. That means conductance values are also functions of phase saturation and change as water saturations change. In the network model, one set of conductance values is obtained for the entire study period.

The influence of relative permeability and viscosity on average reservoir pressure varies with position of flood fronts. Figure 3.14 and Figure 3.15 show that right after breakthrough the effect is more severe, and it reduces towards the end of the study period. To minimize these effects, one needs to find a period in which pressure and saturation vary linearly with injection. That is why mature waterfloods are considered better candidates for the application of these low data requirement tools.

Unlike relative permeability and viscosity, compressibility is not a part of the set of matched parameters. It appears on both sides of the flow equation (formation volume factor, which is a compressibility-dependent parameter, appears on the left-hand side, and compressibility appears on the right) and represent compressibility of all the fluids present in the reservoir. Therefore, an 
Table 3.24: Conductance for single phase homogenous reservoir with SPECSP viscosity $(d . f t / c p)$

\begin{tabular}{ccccc}
\hline & P1 & P2 & P3 & P4 \\
\hline I1 & 1.237 & 1.308 & 0.270 & 0.213 \\
\hline I2 & 1.342 & 0.358 & 1.371 & 0.339 \\
\hline I3 & 1.383 & 1.312 & 1.261 & 1.302 \\
\hline I4 & 0.553 & 1.023 & 0.398 & 1.171 \\
\hline I5 & 0.205 & 0.164 & 0.614 & 0.572 \\
\hline
\end{tabular}

incorrect estimation of the compressibility dramatically influences the parameter estimation (section 3.5.2). When there is a slight difference between compressibility of the fluids in the reservoir, one can use compressibility of one of the fluids. When there is a considerable compressibility difference, total compressibility should be calculated. In well test literature total compressibility is obtained as: (Chaudhry 2004):

$$
c_{t}=S_{o} c_{o}+S_{w} c_{w}+S_{g} c_{g}+c_{f}
$$

The $c_{o}, c_{w}, c_{g}$ and $c_{f}$ terms are the compressibility values for oil, water, gas, and formation, respectively, and $S_{o}, S_{w}$, and $S_{g}$ are oil, water, and gas saturation. $S_{w}$ and $S_{g}$ are obtained by material balance. In an oil-water system, water saturation at each step is obtained from:

$$
S_{w}^{n+1}=S_{w}^{n}+\frac{\left(Q_{i n j}^{n+1}-Q_{\text {prod }}^{n+1} f_{w}^{n+1}\right)}{V_{\text {reservoir }} \phi}
$$

where $f_{w}$ is water cut.

\section{7 $16 \times 25$ Synthetic Example}

To test the performance of the network model on a larger scale, a homogenous field with a larger number of wells was studied. This case has 16 production and 25 injection wells. Wells are positioned on a 5-spot pattern, and the distance between injector-producer well-pairs is $890 \mathrm{ft}$. Injectors are shown by triangles, and producers are represented by circles and are numbered from the top 
left. A history of 65 months, which consists of 1040 data points was generated using the injection history of the example presented in section 3.3.1. Other input parameters are the sames as values used in Section 3.3.1. Tables 3.25 and 3.26 show conductance values and WAFs for the 41 wells in the system. Conductance values shown in Table 3.25 for injectors I7, I8, I14, I17, and I19 are much higher than the rest of the values. Injectors at the corners also demonstrate higher conductance values (Figure 3.16).

Table 3.25: $16 \times 25$ Wells: Conductance $(d . f t / c p)$

\begin{tabular}{|c|c|c|c|c|c|c|c|c|c|c|c|c|c|c|c|c|}
\hline & $P$ & 2 & 3 & P4 & 5 & 6 & 7 & 8 & 9 & 10 & 11 & 12 & 13 & 4 & 15 & P16 \\
\hline I1 & 0.48 & 013 & 0.00 & 000 & 014 & 0 & 0.00 & 0 & 0 & 0 & 0 & 0 & 00 & 0 & 0 & 0.00 \\
\hline I2 & & .38 & .10 & ) & 0.07 & 0.02 & 0.00 & 0.00 & 0.00 & 0 & 0 & 0 & 0 & 0.00 & 0.00 & .00 \\
\hline I3 & 03 & 14 & 13 & 02 & .00 & 0.02 & 0.01 & 0.00 & 0.00 & 0.00 & 0.00 & 0.00 & 0.00 & 0.00 & 0.00 & 0.00 \\
\hline I4 & 0.00 & 0.05 & 17 & 0.19 & 0.00 & 0.00 & 0.03 & 0. & 0.00 & 0.00 & 0.00 & 0.00 & 0.00 & .00 & .00 & .00 \\
\hline I5 & 0.00 & 0.00 & 0.14 & 0.36 & 0.00 & 0.00 & 0.00 & 0.13 & 0.00 & 0.00 & 0.00 & 0.00 & 0.00 & 0.00 & .00 & 0.00 \\
\hline I6 & 14 & 03 & .00 & 0.00 & 0.12 & 0.00 & 0.00 & 0.00 & 0.03 & 0.00 & 0.00 & 0.00 & 0.00 & 0.00 & 0.00 & 0.00 \\
\hline I7 & 77 & .85 & .21 & 0.00 & 6.90 & 6.98 & 1.38 & 0.00 & 3.13 & 1.08 & 0.00 & 0.00 & 0.00 & 0.00 & 0.00 & 0.00 \\
\hline I8 & 1.70 & 8.64 & 8.58 & 3.70 & 0.85 & 7.80 & 8.74 & 2.00 & 0.00 & 2.22 & 0.43 & 0.00 & 0.00 & 0.00 & 0.00 & 0.00 \\
\hline IO & 00 & .02 & .17 & 0.20 & 0.00 & 0.02 & 0.18 & 0.18 & 0.00 & 0.00 & 0.03 & 0.03 & 0.00 & 0.00 & 0.00 & 0.00 \\
\hline I10 & 00 & .00 & .09 & 0.39 & 0.00 & 0.00 & 0.05 & 0.37 & 0.00 & 0.00 & 0.00 & 0.09 & 0.00 & 0.00 & 0.00 & 0.00 \\
\hline I11 & 0.09 & 0.00 & 0.00 & 0.00 & 0.27 & 0.03 & 0.00 & 0.00 & 0.26 & 0.04 & 0.00 & 0.00 & 0.09 & 0.00 & 0.00 & 0.00 \\
\hline $\mathrm{I} 12$ & 0.01 & 0.01 & 0.00 & 0.00 & 0.10 & 0.14 & 0.01 & 0.00 & 0.09 & 0.13 & 0.04 & 0.00 & 0.02 & 0.01 & 00 & 0.00 \\
\hline I13 & 0.00 & 0.00 & 0.00 & 0.00 & 0.02 & 0.06 & 0.07 & 0.02 & 0.01 & 0.07 & 0.09 & 0.00 & 0.00 & 0.01 & 0.02 & 0.00 \\
\hline I14 & 0.00 & 0.00 & 2.46 & 3.54 & 0.00 & 1.39 & 8.77 & 8.77 & 0.00 & 0.34 & 6.93 & 8.77 & 0.00 & 0.00 & 0.11 & 3.02 \\
\hline I15 & .00 & 0.00 & 0.00 & 0.06 & 0.00 & 0.00 & 0.03 & 0.20 & 0.00 & 0.00 & 0.02 & 0.24 & 0.00 & 0.00 & 0 & 0.03 \\
\hline I16 & 0.00 & 0.00 & 0.00 & 0.00 & 0.08 & 0.00 & 0.00 & 0.00 & 0.30 & 0.06 & 0.00 & 0.00 & 0.34 & 0.07 & 0.00 & 0.00 \\
\hline I17 & 0.00 & .00 & 0.00 & 0.00 & 2.94 & 2.49 & 0.00 & 0.00 & 8.75 & 8.41 & 2.15 & 0. & 8.77 & 7.87 & 2.21 & 0.00 \\
\hline $\mathrm{I} 18$ & 0 & 0.00 & 0.00 & 0.0 & 0.00 & 0.02 & 0.02 & $0 .($ & 0.02 & 0.15 & 0. & 0. & 0.07 & 8 & 15 & 0.01 \\
\hline I19 & 0.00 & 0.00 & 0.00 & 0.00 & 0.00 & 0.00 & 0.12 & 0.75 & 0.00 & 2.16 & 6.53 & 7.53 & 0.00 & 3.66 & 8.48 & 8.77 \\
\hline I 20 & 0.00 & 0.00 & 0.00 & 0.00 & 0.00 & 0.00 & 0.00 & 0.03 & 0.00 & 0.00 & 0.01 & 0.12 & 0.00 & 0.00 & 0.03 & 0.13 \\
\hline I21 & 0.00 & 0.00 & 0.00 & 0.00 & 0.00 & 0.00 & 0.00 & 0.00 & 0.06 & 0.00 & 0.00 & 0.00 & 0.19 & 0.08 & 0.00 & 0.00 \\
\hline I 22 & 0.00 & 0.00 & 0.00 & 0.00 & 0.00 & 0.00 & 0.00 & 0.00 & 0.05 & 0.01 & 0.00 & 0.00 & 0.20 & 0.15 & 0.06 & 0.00 \\
\hline I 23 & 0.00 & 0.00 & 0.00 & 0.00 & 0.00 & 0.00 & 0.00 & 0.00 & 0.00 & 0.02 & 0.04 & 0.00 & 0.07 & 0.23 & 0.22 & 0.08 \\
\hline $\mathrm{I} 24$ & 0.00 & 0.00 & 0.00 & 0.00 & 0.00 & 0.00 & 0.00 & 0.00 & 0.00 & 0.00 & 0.03 & 0.06 & 0.00 & 0.06 & 0.28 & 0.36 \\
\hline I 25 & 00 & 00 & 00 & 00 & 00 & 0 & 0 & 0.00 & 0.00 & 0.00 & 0.00 & 0.13 & 0.00 & 0.00 & 0.11 & 0.45 \\
\hline
\end{tabular}


Table 3.26: $16 \times 25$ Wells: Average well allocation factors

\begin{tabular}{|c|c|c|c|c|c|c|c|c|c|c|c|c|c|c|c|c|}
\hline & P1 & P2 & P3 & P4 & P5 & P6 & P7 & P8 & P9 & P10 & P11 & P12 & P13 & P14 & P15 & P16 \\
\hline I1 & 64 & 0.18 & 00 & 00 & 8 & 00 & 0 & 0 & 00 & .00 & 00 & .00 & .00 & .00 & .00 & 0.00 \\
\hline I2 & 0.40 & 040 & 0.11 & 0.00 & 0.07 & 0.02 & 0.00 & 0.00 & 0.00 & 0 & 0 & 0.00 & 0 & 0 & 0.00 & 00 \\
\hline I3 & 0.09 & 39 & 37 & $0 /$ & 0.00 & 0.05 & 0.03 & 0.00 & 0.00 & 0.00 & 0.00 & 0.00 & 0.00 & 0.00 & 0 & 0.00 \\
\hline I4 & 0 & 0.11 & 36 & 30 & 00 & 0.00 & 0.06 & 0.09 & 0.00 & 0.00 & 0.00 & 0.00 & 0.00 & 0.00 & 0.00 & 0.00 \\
\hline I5 & 0.0 & 0.00 & 0.22 & 0.57 & 0.00 & 0.00 & 0.00 & 0.21 & 0.00 & 0.00 & 0.00 & 0.00 & 0.00 & 0.00 & 0.00 & 0.00 \\
\hline I6 & 0.44 & 08 & 0.00 & 0.00 & 0.37 & 0.02 & 0.00 & 0.00 & 0.10 & 0.00 & 0.00 & 0.00 & 0.00 & 0.00 & 0.00 & 0.00 \\
\hline I7 & 0.07 & 0.19 & 0.00 & 0.00 & 0.20 & 0.34 & 0. & 0. & 0.09 & 0.05 & 0.00 & 0.00 & 0.00 & 0.00 & 0.00 & 0.00 \\
\hline I8 & 0.01 & 0.13 & 0.08 & 0.01 & 0.02 & 0.30 & 0.31 & 0.03 & 0.00 & 0.08 & 0.02 & 0.00 & 0.00 & 0.00 & 0.00 & 0.00 \\
\hline I9 & 00 & 02 & 0.21 & 0.23 & 0.00 & 0.03 & 0.22 & 0.21 & 0.00 & 0.00 & 0.03 & 0.04 & 0.00 & 0.00 & 0 & 0.00 \\
\hline I10 & 0.00 & 0.00 & 09 & .59 & 0.00 & 0.00 & 0.06 & 0.37 & 0.00 & 0.00 & 0.00 & 0.09 & 0.00 & 0 & 0.00 & .00 \\
\hline I11 & 0.11 & 0.00 & 0.00 & 0.00 & 0.35 & 0.04 & 0.00 & 0.00 & 0.34 & 0.05 & 0.00 & 0.00 & 0.11 & 0.00 & 0.00 & 0.00 \\
\hline I12 & 00 & 00 & 0.00 & 0.00 & 0.17 & 0.26 & 0.02 & 0.00 & 0.17 & 0.23 & 0.07 & 0.00 & 0.03 & 2 & 0.00 & 0.00 \\
\hline I13 & 0.00 & .00 & 0.01 & 0.00 & 0.05 & 0.17 & 0.20 & 0.05 & 0.03 & 0.18 & 0.23 & 0.01 & 0.00 & 0.02 & 0. & 0.00 \\
\hline I14 & 0.00 & 0.00 & 0.03 & 0.01 & 0.00 & 0.05 & 0.32 & 0.11 & 0.00 & 0.01 & 0.27 & 0.18 & 0.00 & 0.00 & 0.00 & 0.02 \\
\hline I15 & 0.00 & 0.00 & 0.00 & 0.11 & 0.00 & 0 & 0.05 & 0. & 0. & 0.00 & 0. & 0.41 & 0 & 0 & 0.00 & 0.05 \\
\hline I16 & 0.00 & 0.00 & 0.00 & 0.00 & 0.10 & 0.00 & 0.00 & 0.00 & 0.36 & 0.07 & 0.00 & 0.00 & 0.40 & 0.08 & 0.00 & 0.00 \\
\hline I17 & 0.0 & 0.00 & 0.00 & 0.00 & 0.07 & 0.09 & 0.00 & 0.00 & 0.19 & 0.28 & 0.07 & 0.00 & 0.10 & 0.16 & 0. & 0.00 \\
\hline I18 & 0.00 & 0.00 & 0.00 & 0 & 0. & 0. & 0.02 & 0. & 0. & 0 & 1 & 0.01 & 0.09 & 0.22 & 0.19 & 0.01 \\
\hline I19 & 0.00 & 0.00 & 0.00 & 0.00 & 0.00 & 0.00 & 0.01 & 0.02 & 0.00 & 0.09 & 0.29 & 0.20 & 0.00 & 0.10 & 0.21 & 0.08 \\
\hline I 20 & 0.0 & 0 & 0.00 & 0.00 & 0.00 & 0.00 & 0.00 & 0.09 & 0.00 & 0.00 & 0.04 & 0.38 & 0.00 & 0.00 & 0. & 0.41 \\
\hline $\mathrm{I} 21$ & 0.00 & 0.00 & 0.00 & 0.00 & 0.00 & 0.00 & 0.00 & 0.00 & 0.18 & 0.00 & 0.00 & 0.00 & 0.59 & 0.23 & 0.00 & 0.00 \\
\hline I 22 & 0.00 & 0.00 & 0.00 & 0.00 & 0.00 & 0.00 & 0.00 & 0.00 & 0.10 & 0.03 & 0.00 & 0.00 & 0.43 & 0.32 & 0.13 & 0.00 \\
\hline I 23 & 0.00 & 0.00 & 0.00 & 0.00 & 0.00 & 0.00 & 0.00 & 0.00 & 0.0 & 0.03 & 0.06 & 0.00 & 0.10 & 0.35 & 0.34 & 0.12 \\
\hline I 24 & 0.00 & 0.00 & 0.00 & 0.00 & 0.00 & 0.00 & 0.00 & 0.00 & 0.00 & 0.00 & 0.04 & 0.08 & 0.00 & 0.07 & 0.35 & 0.46 \\
\hline I 25 & .00 & 00 & 00 & 0 & 0 & 0 & 0.00 & 0.00 & 0.00 & 0.00 & 0.00 & 0.19 & 0.00 & 0.00 & 0.16 & 0.65 \\
\hline
\end{tabular}

\subsection{Conclusion}

In this research reservoirs are modeled as a number of nodes containing a well. Flow between nodes is through bonds or throats, which are controlled by conductance between nodes. Conductance is directly proportional to effective permeability and cross-sectional area open to flow between wells and inversely related to the distance between them. To obtain conductance values, three classes of data are required. The first and main group is production and injection data and well position, 


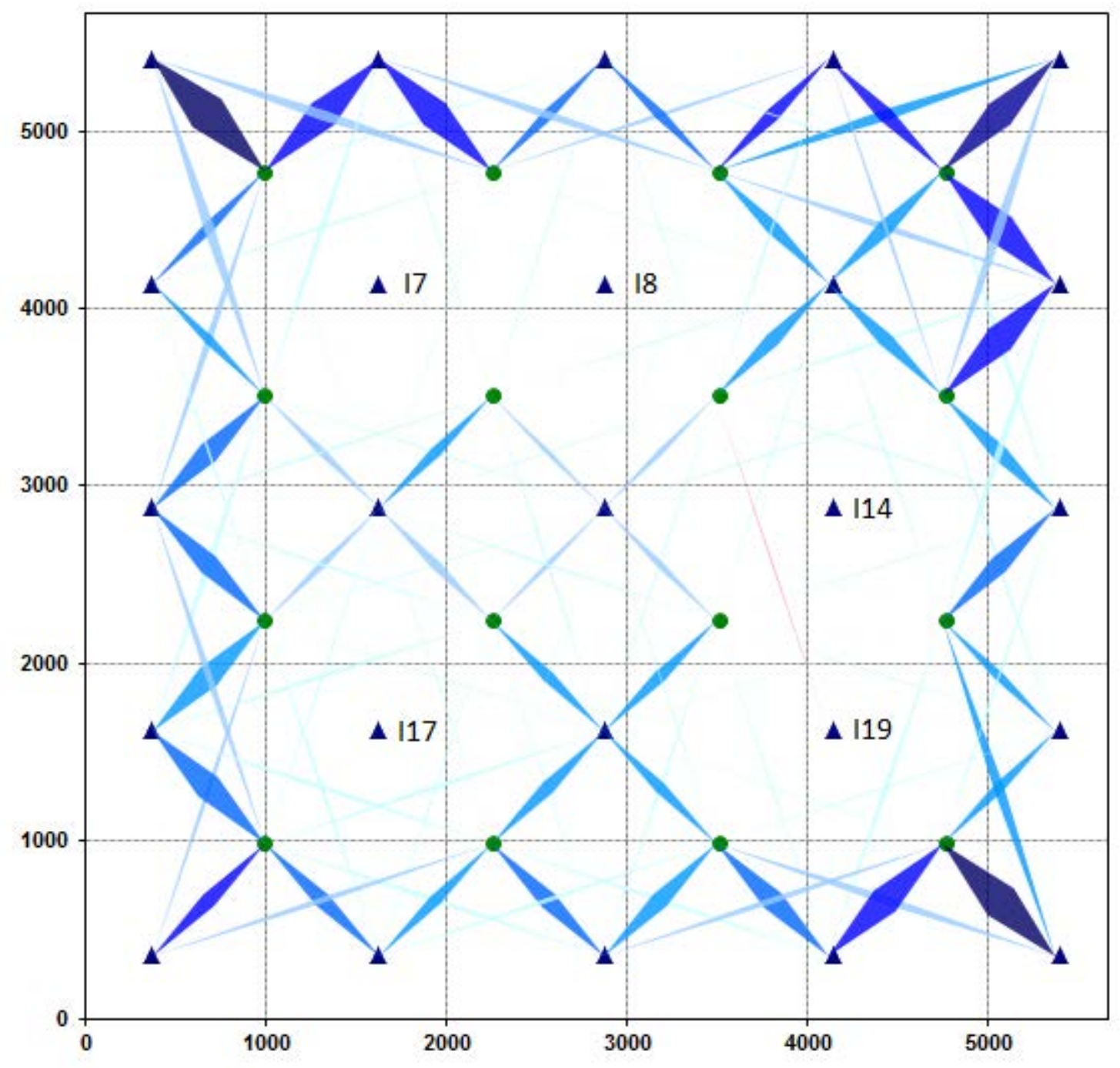

Figure 3.16: $16 \times 25$ wells: Conductance and flux

which are usually available with some degree of certainty. The second group are those data for which rough estimates of the actual values suffice. They include the location of the boundary of the reservoir, average porosity, bottomhole pressures, and productivity indicies of the wells. The third group are those that are not necessary, but considering them improves the quality of the result. For example, including the fault position in the process of assigning volume to each well helps to obtain more realistic node volumes. Adding this information improves the result.

The network model was validated by showing that conductance values obtained are proportional to average reservoir permeability. It also was validated by application to synthetic fields by showing 
that the network model captures barriers, flow paths, and permeability trends. The technique was tested in cases like shutting in wells, and changing flow pattern and solutions to handle each case was provided. It also was tested for situations in which incorrect input parameters are used. The results show that incorrect values of BHP and WI give correct values of WAFs, but the error will be reflected in local values of conductance. Errors in estimating reservoir volume and compressibility will be more widespread and influence the entire set of conductance values and WAFs.

The method was also tested to see if it handles changes in pressure and saturation properties, like relative permeability and viscosity. These properties alter resistance to flow. Their influence is reflected in the obtained conductance values.

The method is fast and can be easily set up. It resolves some of the limitations that currently available methods have. It calculates conductance values, which have a physical meaning that still must be interpreted. This approach considers changes in flow pattern due to shuting-in a producer for a long time, adding or removing wells, or changing well rates. This allows the method to study a broader time span. It also allows testing a wider range of injection-production scenarios in the prediction phase. 


\section{Chapter 4}

\section{Single Phase Model with Injector-Injector Interactions}

Incompatibility between results from connectivity models on the one hand and streamline approaches and tracer tests on the other hand has been addressed by Albertoni and Lake (2003) and Shahvali et al. (2012). Usually connectivity models show a stronger connection between nonadjacent injector-producer pairs than streamline approaches do. Albertoni and Lake (2003) simulated a tracer test on the homogeneous case shown in Section 3.3.1 and obtained the fraction of tracer injected in the $i$ th injector that was produced in the $j$ th producer. They found that none of the tracer injected in I4 was produced by P1, but connectivity weights show $17 \%$ influence of I4 on P1. They stated that tracer tests show which producer produces the injected water, so tracer weights depend on injection rates. On the other hand, connectivity coefficients demonstrate the influence of injectors on each producer. The result of a streamline simulation with FrontSim ${ }^{\circledR}$ software also shows tracer results and show a 0 allocation fraction for non-adjacent injector-producer pairs for this example. Shahvali et al. (2012) evaluated Capacitance-Resistance Model (CRM) and streamline (SL) approaches on a sector model representing a mature waterflood. They compared injection allocation factors of both approaches over three years of history. When injector-producer pairs show a very low or very high connection, there is good agreement between the results of both methods. But for an intermediate range of allocation factors, there is a conflict between results of CRM and SL.

In addition to the degree of influence of injectors on producers, there is another discrepancy in the results of the network model approach that indicate a need for modification. As was noted previously, the synthetic homogeneous model that was studied in Section 3.3.1 is a single layer system. In this model, injection fluctuates but does not stop in any well; therefore there is a constant flow from injectors to producers. If one traces streamlines between wells, adjacent injectors 
and producers are always connected by streamlines, although their density changes as rates fluctuate. Flow between wells is shown in Figure 3.1 where there apears to be a substantial amount of fluid flowing between non-adjacent injector-producer pairs. The impression that a graphic like this presents is that flow paths between injectors and producers seem to cross. This seems to require the fluid to be moving past strongly connected injector-producer pairs (the flow seems to be crossing streamlines). This should not happen in a single-layered system. For instance, in Figure 3.1 there is a considerable amount of flow between nonadjacent I4 and P1. But I3 and P2 are connected by a stronger connection, and flow between P1-I4 requires crossing this strong connection.

From this example, one can infer that there may be an influence between nonadjacent injectionproduction pairs, but this influence should not be represented by flow between them, because it is inconsistent with the streamlines that are present. The missing part of this influence chain is injector-injector interactions. As the rate of injector $i$ fluctuates, the pressure field around injector $i$ changes. This change influences the pressure field around both producers and injectors adjacent to injector $i$. This leads to changes in flow from adjacent injectors to connected producers, since flow is proportional to pressure gradient. This means injector $i$ indirectly influences producers connected to adjacent injectors, and this influence is imposed by the pressure field.

This chapter proposes to include injector interactions in obtaining conductance values. Cases that were evaluated in the previous chapter are evaluated again to see whether adding these new connections helps to obtain a more viable solution. In addition, some new cases with different permeability trends are tested with the modified approach.

\subsection{Including injector-injector intraction in the network model}

The simplest way to include injector interactions in the network model is to connect volumes containing injectors. In other words, bonds are considered between source volumes in addition to bonds between source and sink volumes. The injector-injector bonds, have the same properties as injector-producer bonds but a smaller radius of investigation is used, and as a result a smaller coordination number is considered for injector-injector connections. It is expected that these new 
bonds would help to make a more realistic representation of flow in the reservoir. That is how they were supposed to work: when injection rate at injector $i$ fluctuates, the pressure of node volume $i$ changes, which influences flux from injector $i$ not only to connected producers but also to connected injectors. Flow from injector $i$ to connected injectors changes the associated node volume pressures of those injectors which, consequently change flow from those injectors to connected producers. Therefore injector $i$ influences producers connected to other injectors through injectorinjector bonds.

\subsection{Application of the model with injector-injector interaction}

\subsubsection{Homogeneous case}

The first candidate to test the modified model on is the homogeneous case described in Section 3.5. Four additional bonds were added to the 20 original bonds, which increased the number of unknowns to 24 . These new bonds connect I3 to I1, I2, I4 and I5. The same injection history is applied, and output of the model is matched with the synthetic model total fluid production.

In the conductance values table, new parameters are added to account for the injector-injector interactions. Table 4.1 shows the conductance values obtained for the homogeneous case. Conductance values are plotted in Figure 4.1 a. Gray diamonds represent bonds connecting injectors. They are comparatively larger than injector-producer conductance values. One explanation could be that since the pressure differences between injector-injector pairs are comparatively smaller than injector-producer pairs, a larger conductance value is required for fluid flow between those pairs. It could also be the effect of factors that influence the flow but are ignored in the model such as producer-producer interaction. $k A_{c s}$ and flow between wells are shown in Tables 4.2 and 4.3. Well allocation factors, which are the fraction of the flow from injectors to connected volumes, are given in Table 4.4, and Figure $4.1 \mathrm{~b}$ illustrates these values. Flow between injector-producer pairs is depicted by complete diamonds, and flow between injector-injector pairs by half diamonds. For example, there are two half diamonds between I1 and I3; one starts from I1, and the other starts from I3. The first one, which is thicker shows flow from I1 to I3, and the latter shows flow from 
Table 4.1: Homogenous Reservoir: Conductance values $(d . f t / c p)$

\begin{tabular}{cccccccccc}
\hline & P1 & P2 & P3 & P4 & I1 & I2 & I3 & I4 & I5 \\
\hline I1 & 0.995 & 0.997 & 0.001 & 0.000 & 0.000 & 0.000 & 2.295 & 0.000 & 0.000 \\
\hline I2 & 0.890 & 0.000 & 0.887 & 0.001 & 0.000 & 0.000 & 2.030 & 0.000 & 0.000 \\
\hline I3 & 0.399 & 0.369 & 0.433 & 0.403 & 2.295 & 2.030 & 0.000 & 2.198 & 2.186 \\
\hline I4 & 0.001 & 0.943 & 0.001 & 0.938 & 0.000 & 0.000 & 2.198 & 0.000 & 0.000 \\
\hline I5 & 0.001 & 0.001 & 0.939 & 0.942 & 0.000 & 0.000 & 2.186 & 0.000 & 0.000 \\
\hline
\end{tabular}

Table 4.2: Homogenous Reservoir: $k A$ values $\left(d . f t^{2}\right)$

\begin{tabular}{cccccccccc}
\hline & P1 & P2 & P3 & P4 & I1 & I2 & I3 & I4 & I5 \\
\hline I1 & 795.9 & 797.9 & 2 & 0.3 & 0 & 0 & 2596.9 & 0 & 0 \\
\hline I2 & 712.3 & 0.2 & 709.8 & 1.4 & 0 & 0 & 2296.4 & 0 & 0 \\
\hline I3 & 319.1 & 294.9 & 346.7 & 322.7 & 2596.9 & 2296.4 & 0 & 2486.5 & 2473 \\
\hline I4 & 1 & 754 & 1.8 & 750.6 & 0 & 0 & 2486.5 & 0 & 0 \\
\hline I5 & 1.4 & 1.1 & 750.9 & 753.8 & 0 & 0 & 2473 & 0 & 0 \\
\hline
\end{tabular}

I3 to I1. Once again, flow occurs between the volumes containing injectors but not between the injectors. From Figure $4.1 \mathrm{~b}$ one can infer that the northern part of the system is more pressurized due to higher injection since the diamonds are thicker in the downward direction.

Table 4.3: Homogenous Reservoir: Cumulative flow between Wells $(b b l)$

\begin{tabular}{cccccccccc}
\hline & P1 & P2 & P3 & P4 & I1 & I2 & I3 & I4 & I5 \\
\hline I1 & 67522 & 69650 & 81 & 12 & 0 & 0 & 29609 & 0 & 0 \\
\hline I2 & 54182 & 9 & 57058 & 53 & 0 & 0 & 21641 & 0 & 0 \\
\hline I3 & 22194 & 21240 & 25613 & 24630 & 1554 & 11064 & 0 & 24994 & 17348 \\
\hline I4 & 27 & 46574 & 51 & 49602 & 0 & 0 & 6977 & 0 & 0 \\
\hline I5 & 40 & 32 & 49850 & 51897 & 0 & 0 & 4263 & 0 & 0 \\
\hline
\end{tabular}




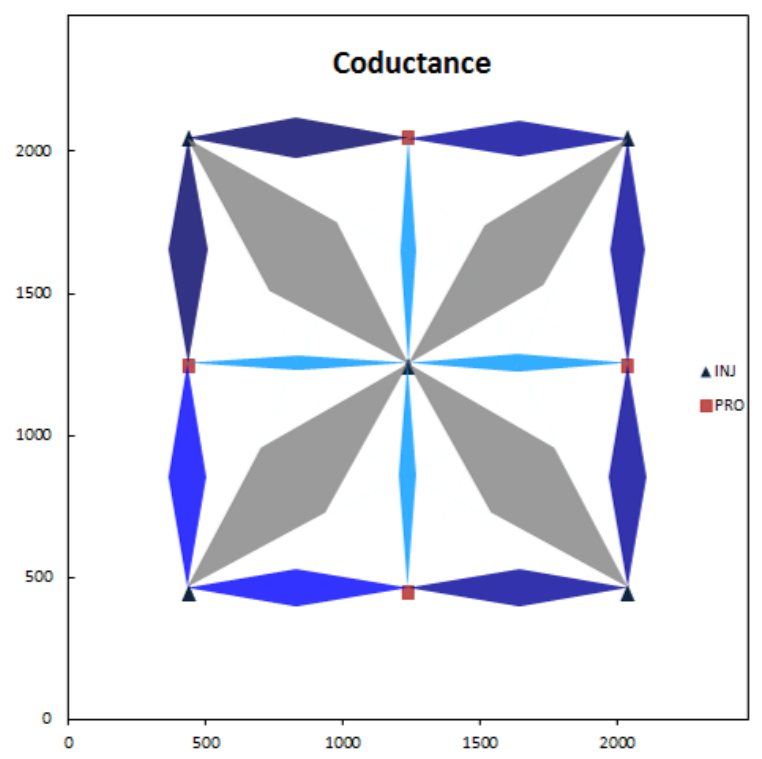

(a) Conductance values.

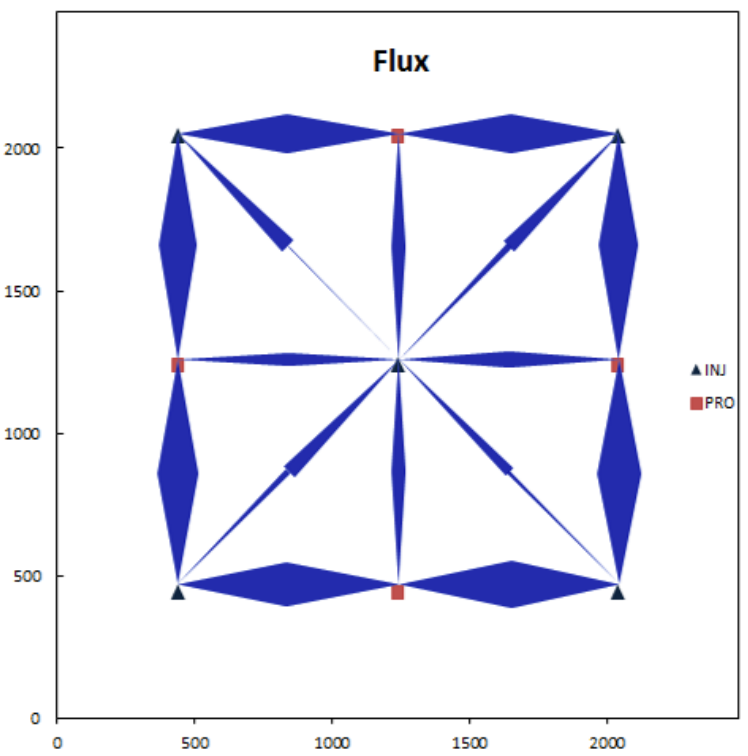

(b) Well allocation factor.

Figure 4.1: Model parameters for a homogeneous reservoir.

Table 4.4: Homogenous Reservoir: Average well allocation factors

\begin{tabular}{cccccccccc}
\hline & P1 & P2 & P3 & P4 & I1 & I2 & I3 & I4 & I5 \\
\hline I1 & 0.405 & 0.417 & 0.000 & 0.000 & 0.000 & 0.000 & 0.177 & 0.000 & 0.000 \\
\hline I2 & 0.408 & 0.000 & 0.429 & 0.000 & 0.000 & 0.000 & 0.163 & 0.000 & 0.000 \\
\hline I3 & 0.149 & 0.143 & 0.172 & 0.166 & 0.010 & 0.074 & 0.000 & 0.168 & 0.117 \\
\hline I4 & 0.000 & 0.451 & 0.000 & 0.480 & 0.000 & 0.000 & 0.068 & 0.000 & 0.000 \\
\hline I5 & 0.000 & 0.000 & 0.470 & 0.489 & 0.000 & 0.000 & 0.040 & 0.000 & 0.000 \\
\hline
\end{tabular}

\subsubsection{Directional permeability trend}

In this section, cases with different directional permeability trends are evaluated to test the modified approach with heterogeneous cases. The permeability distributions were generated by using the GSLIB software package (Deutsch and Journal 1998). Horizontal and vertical permeability trends were generated using sequential Gaussian simulation with 1240-ft correlation length (about $\frac{1}{2}$ the model distance) in the primary direction and 120-ft correlation length in the opposite direction. The mean permeability in all cases and the injection history and input parameters were the same as the parameters used in section 3.5. 
The first two cases show X-direction and Y-direction permeability trends. Permeability distributions are depicted in Figure 4.2 and Figure 4.3. In the X-direction permeability trend case (Figure 4.2), conductance values and WAFs (Figure $4.4 a$ and $b$ ) are large for connections that connect injector 3 to producers 2 and 3. Producers 1 and 4 are positioned in lower permeability regions, and that is why there isn't much flow from adjacent injectors to these wells. Conductance values and WAFs that are shown in Figure $4.5 a$ and $b$ show very strong connections in the Y-direction which is consistant with the assumed Y-direction permeability trend (Figure 4.3).

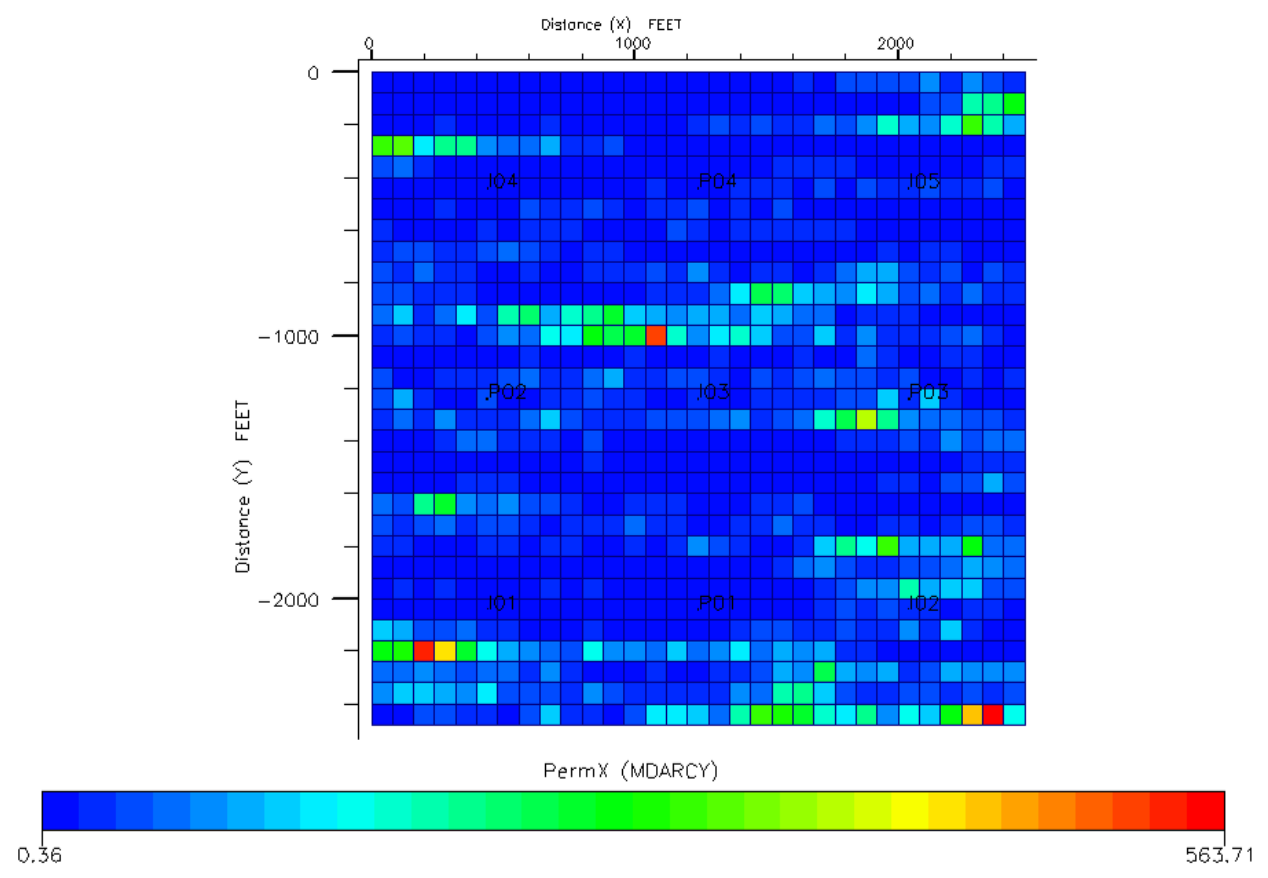

Figure 4.2: X-direction permeability trend

Figure 4.6 and 4.7 show the permeability distribution for cases with NW-SE and SW-NE permeability trends. What differentiates the conductance values and WAFs shown in Figure $4.8 a$ and $b$ and Figure $4.9 a$ and $b$ from vertical and horizontal permeability trend cases is the influence of the injectors. In both cases injector-injector connections that are in the direction of the permeability trend are highlighted. The last case has an omni-direction permeability trend (Figure 4.10). Conductance values and WAFs shown in Figure $4.11 a$ and $b$ do not demonstrate any specific permeability trend. 


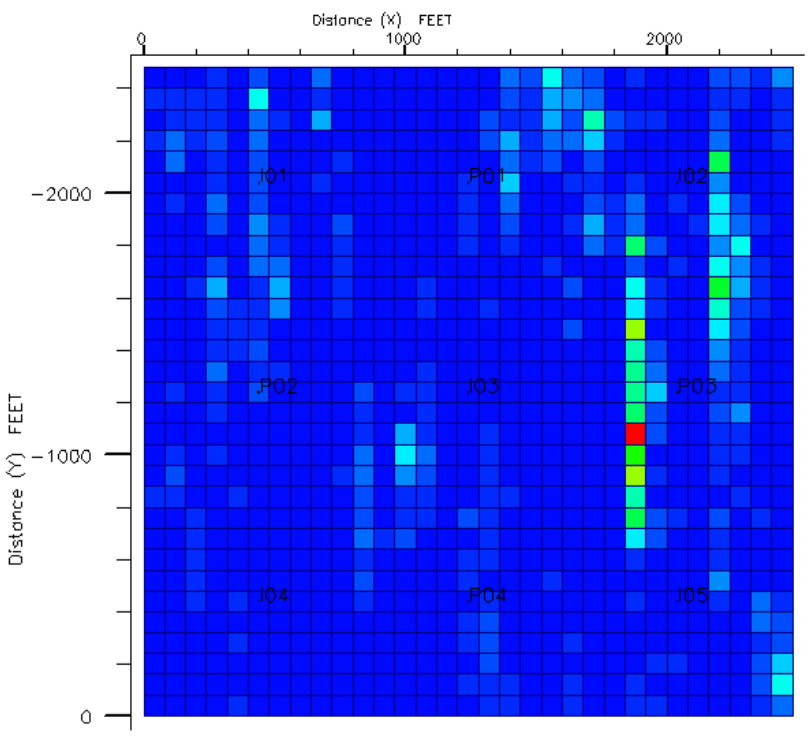

PermX (MDARCY)

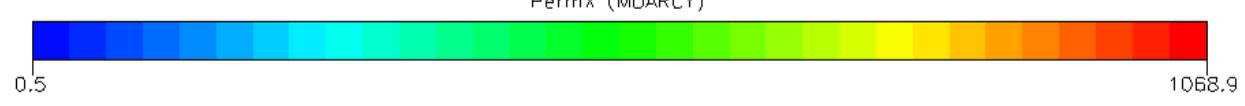

Figure 4.3: Y-direction permeability trend

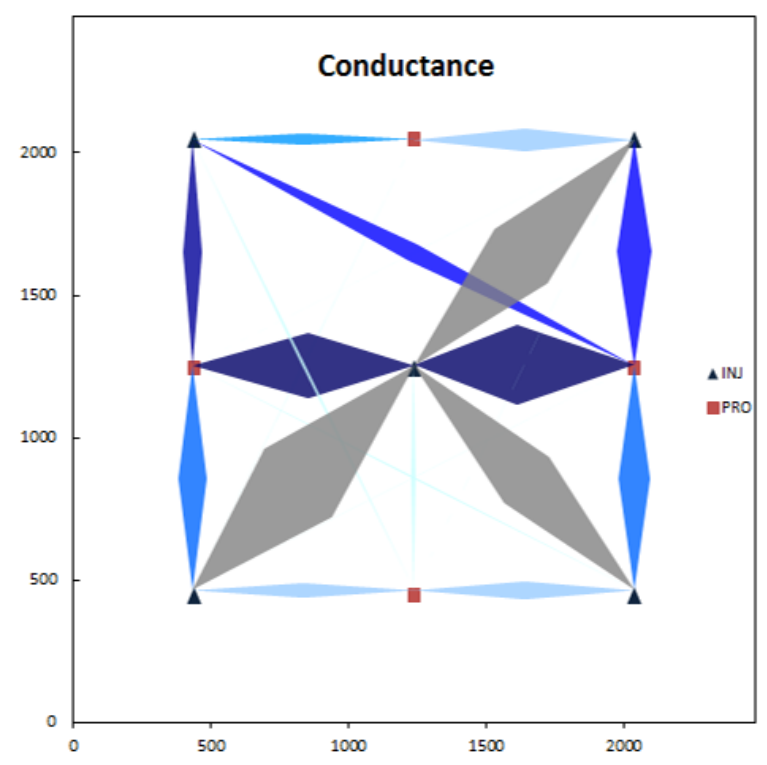

(a) Conductance values.

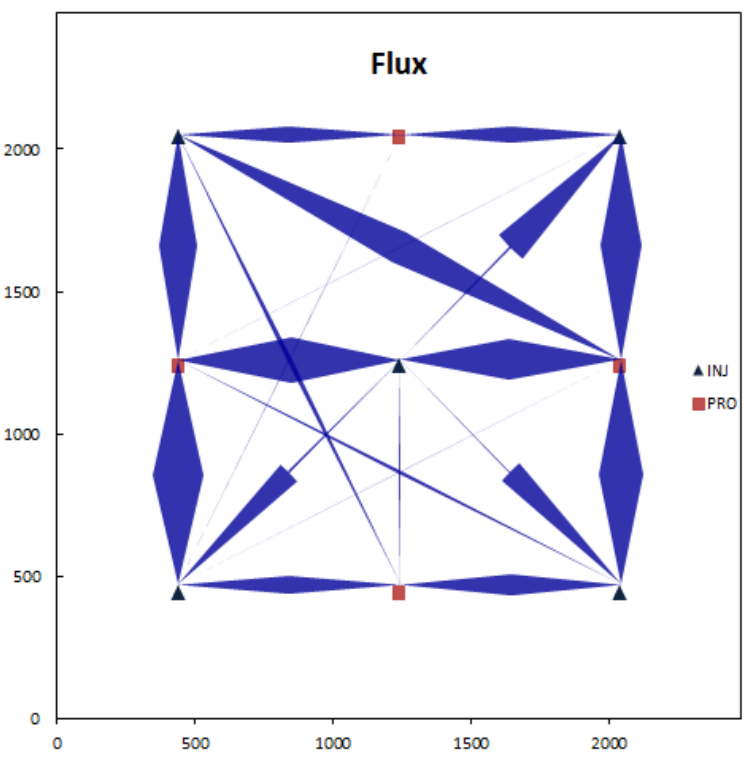

(b) Well allocation factor.

Figure 4.4: Model parameters for X-direction permeability trend.

\subsubsection{Complex geological features}

In section 3.3.6 the network model was tested on a system with a high permeablility channel. The goal was to see if conductance values reflect the high permeablility channel. In this section, the 


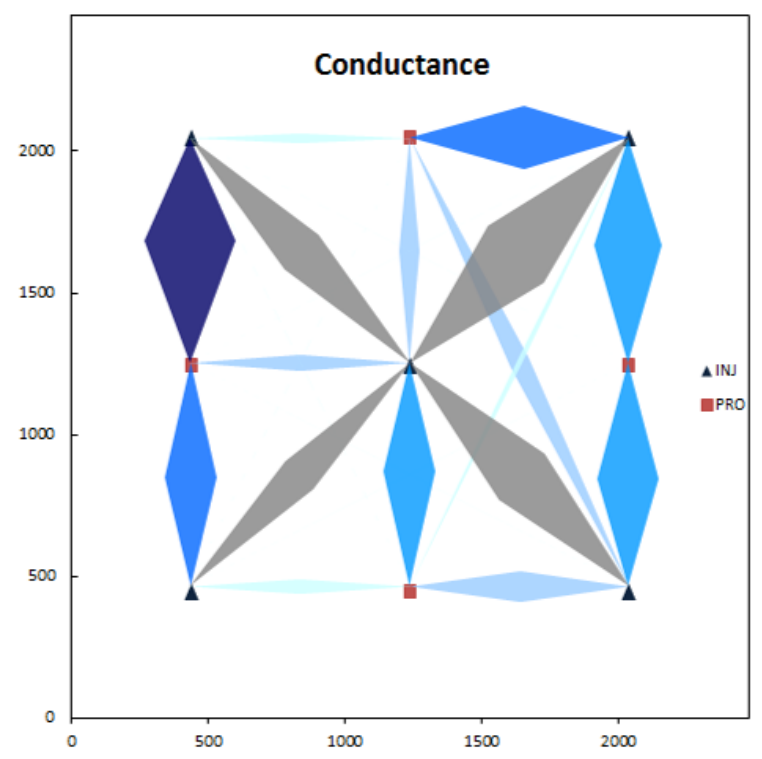

(a) Conductance values.

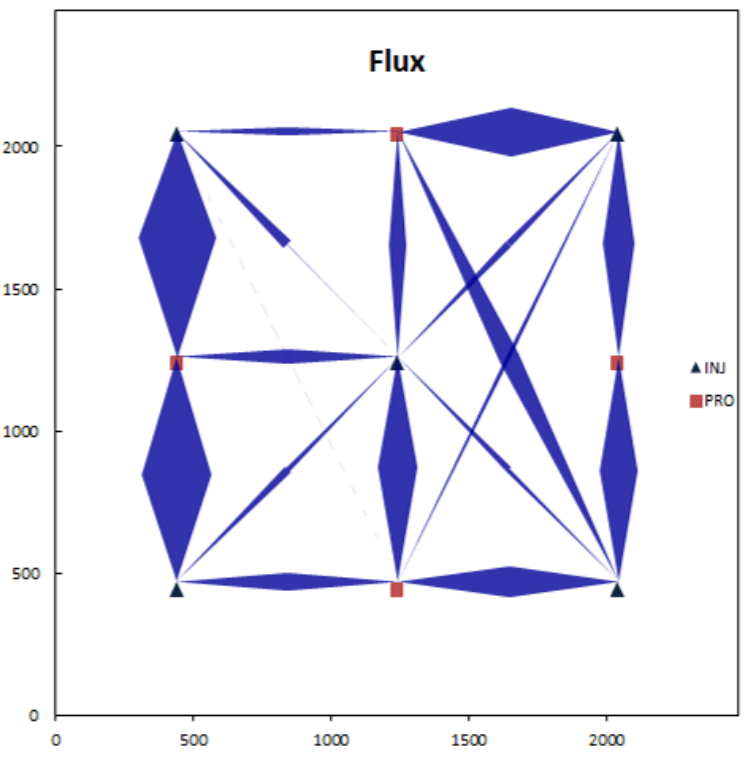

(b) Well allocation factor.

Figure 4.5: Model parameters for Y-direction permeability trend.

same example is used to find out if considering injector-injector interaction reveals more information about the system.

Obtained conductance values and WAFs are illustrated in Figure $4.12 a$ and $b$. All adjacent injectors, injectors 1,3 , and 4 , have strong connections with producer 2 , which is located in the high permeablility channel. The non-adjacent injector 2, which is connected to producer 2 through the channel, also has a strong connection. On the other hand, the distant injector 5 has a strong influence on injector 3. Since all the diamonds are pointed toward the producer 2 , it is easy to infer that injectors are supporting that specific producer. It is also obvious that injectors 2 and 5 are communicating with producers in a different way. Direct connection between injector 2 and producer 2 can be through a high-permeability flow path. But there is no such direct connection for injector 5, and it contributes indirectly.

Unlike the result in section 3.3.6, which did not differentiate between the way that injector 2 and 5 communicate with producer 2, considering injection-injection interaction distinguishes the connection through the high-permeablility channel. This shows that considering injector-injector interaction helps to reveal more information about reservoir features. 


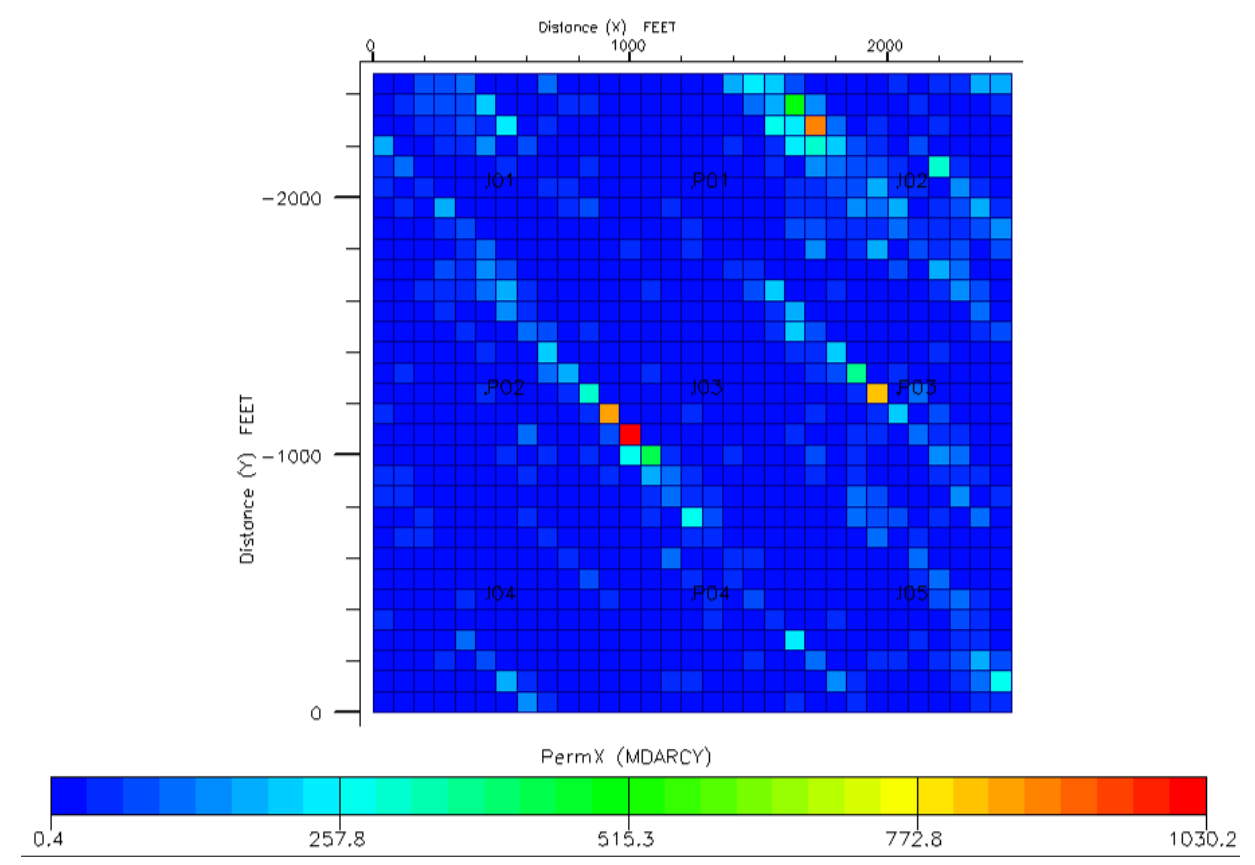

Figure 4.6: NW-SE permeability trend

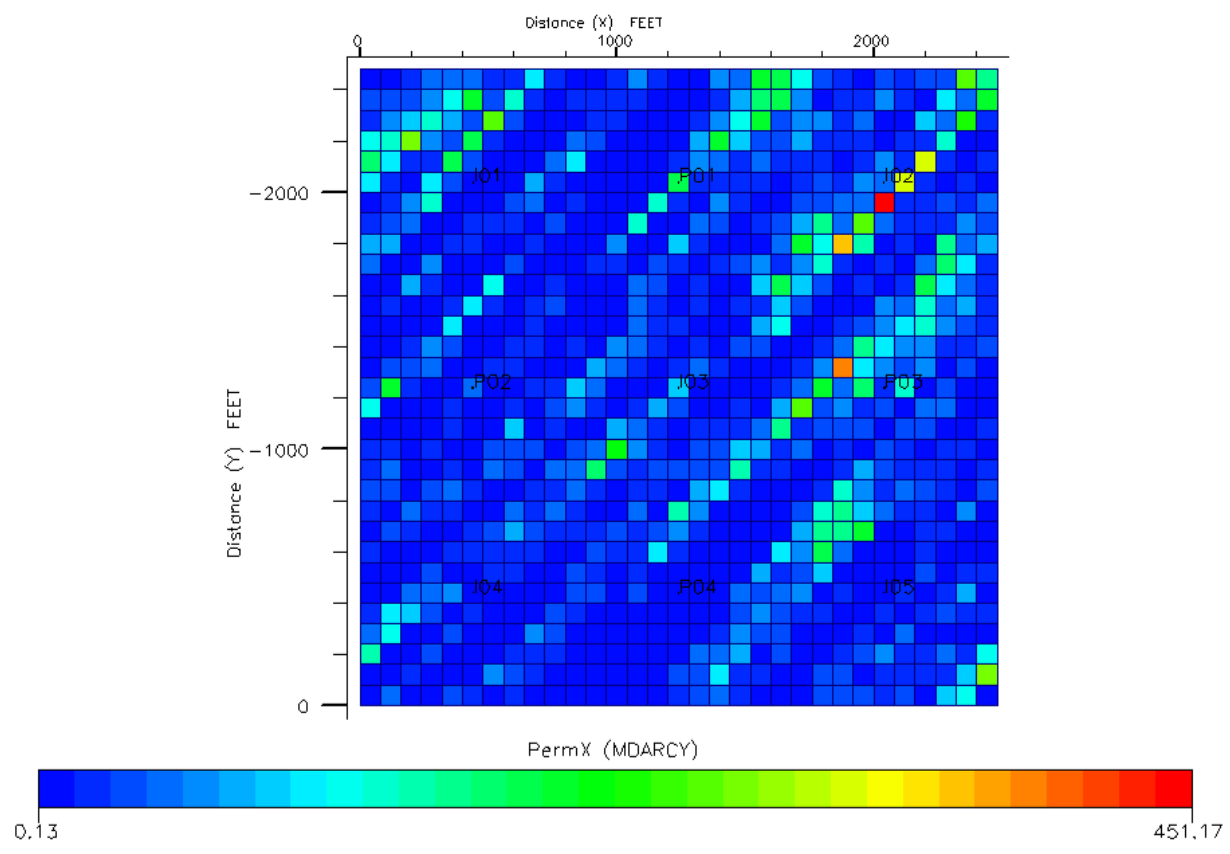

Figure 4.7: SW-NE permeability trend

\subsection{4 $16 \times 25$ Synthetic Field}

In section 3.7 a case with 16 producers and 25 injectors was evaluated to test the performance of the model for a larger number of wells. Symmetric conductance values were obtained for each 


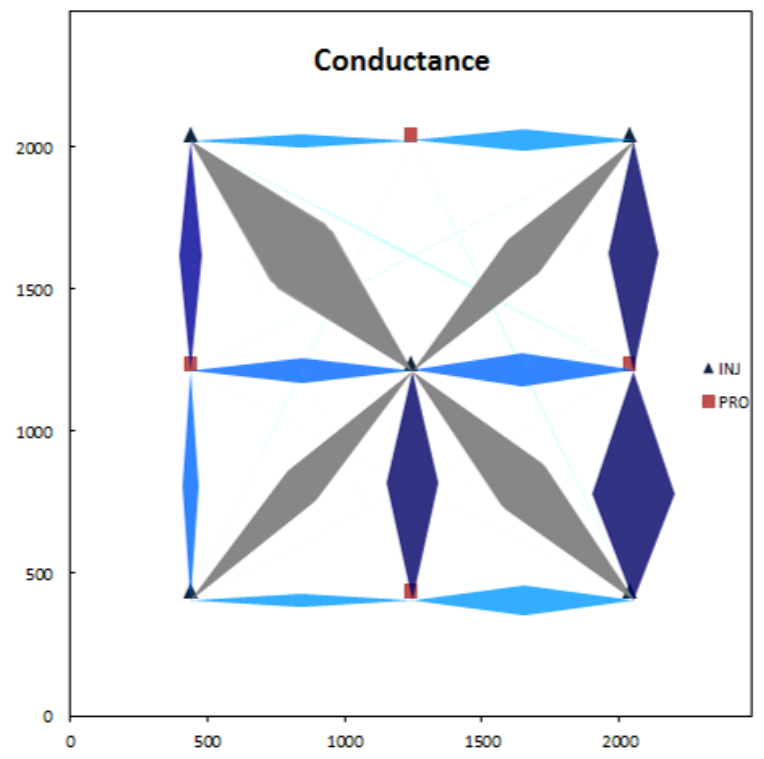

(a) Conductance values.

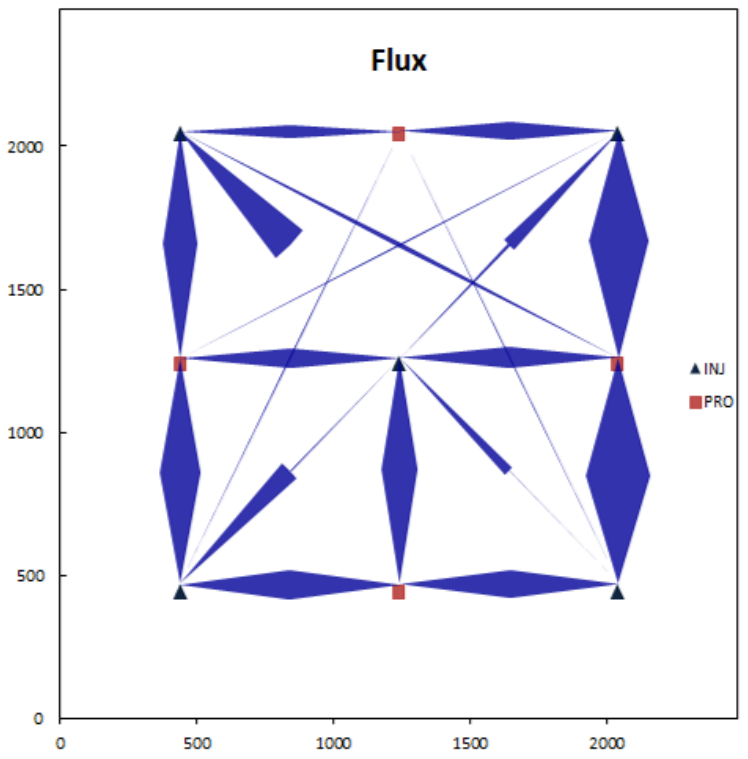

(b) Well allocation factor.

Figure 4.8: Model parameters for NE-SW permeability trend.

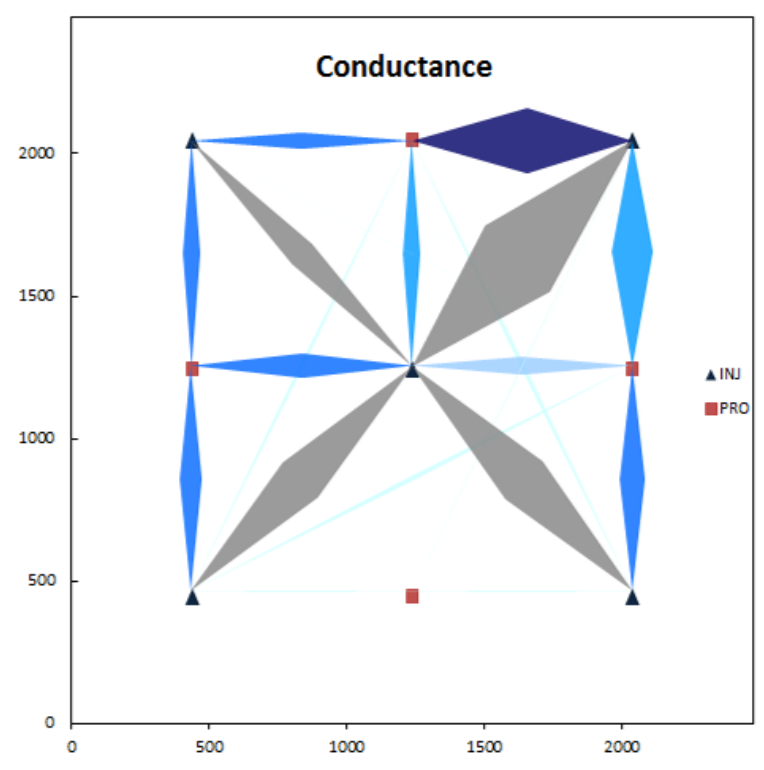

(a) Conductance values.

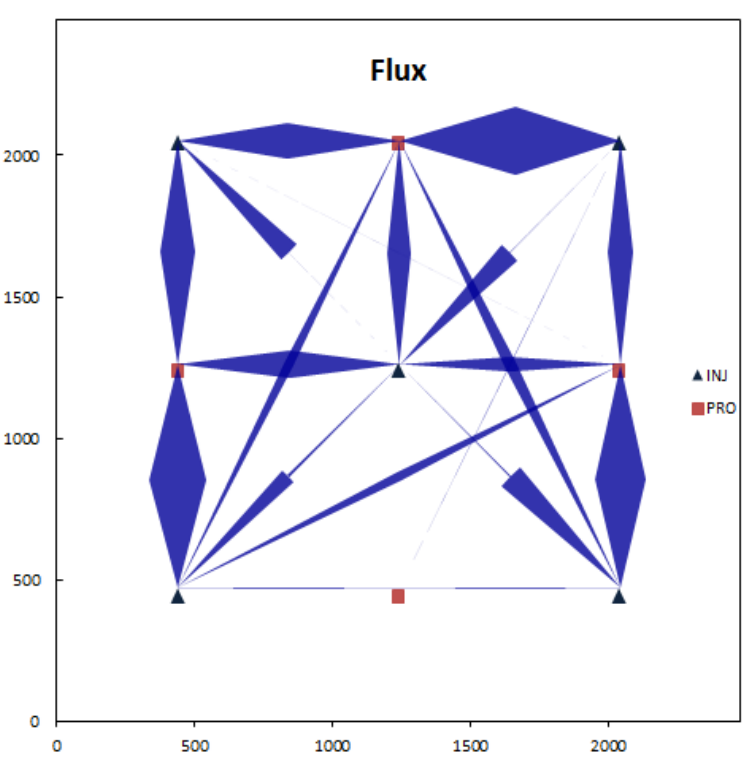

(b) Well allocation factor.

Figure 4.9: Model parameters for SW-NE permeability trend.

injector, although values of conductance were different from injector to injector. In addition, some injectors showed very large conductance values. In this section, the same example is evaluated with the network model that considers injector-injector interaction to see if the result reflects the homogeneous nature of the system. The conductance values and WAFs are presented in Figure 4.13 


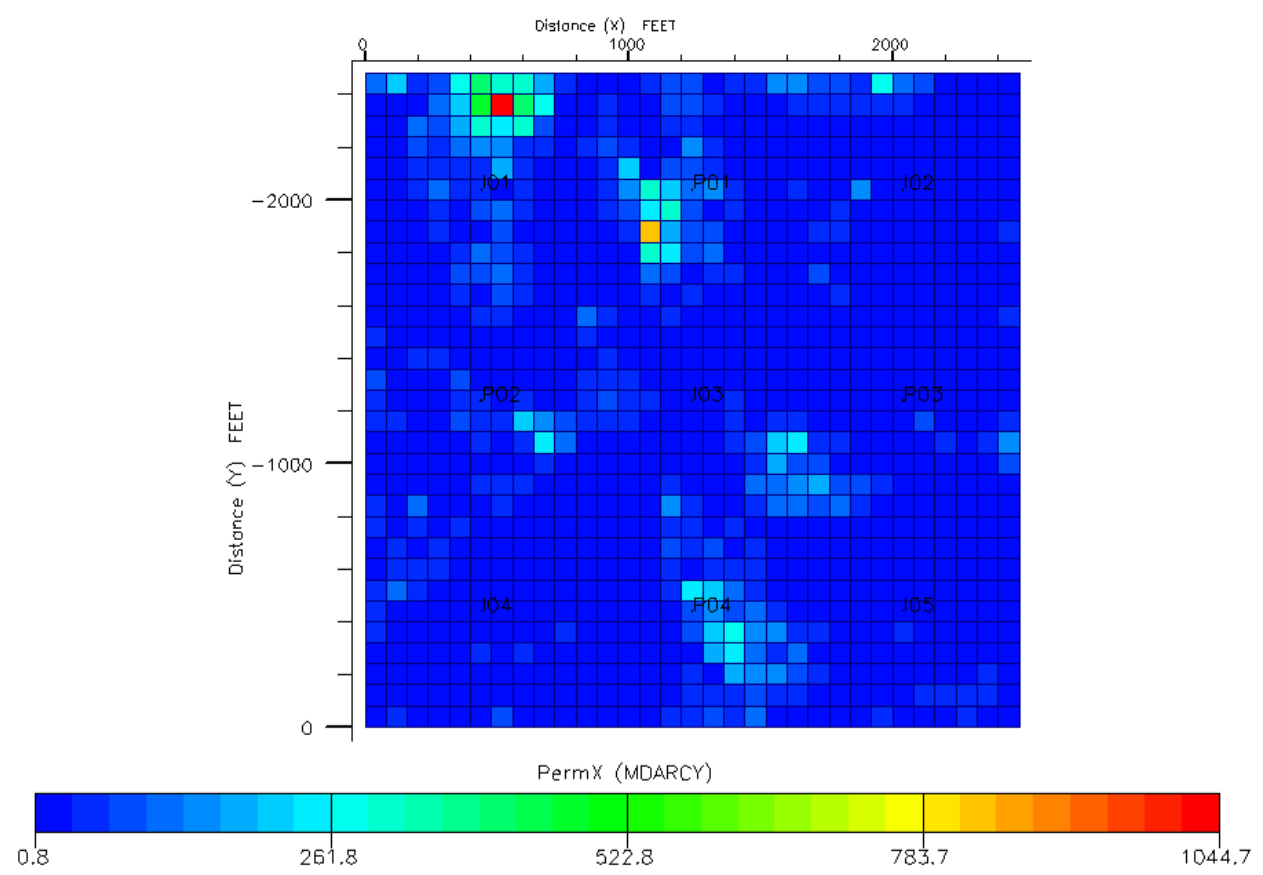

Figure 4.10: Omni-direction permeability trend

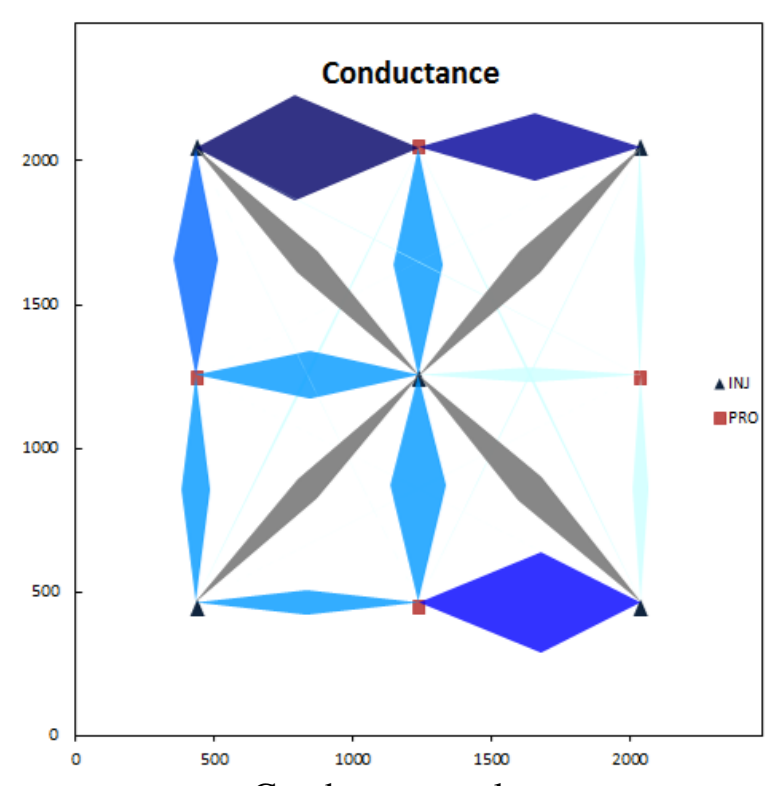

(a) Conductance values.

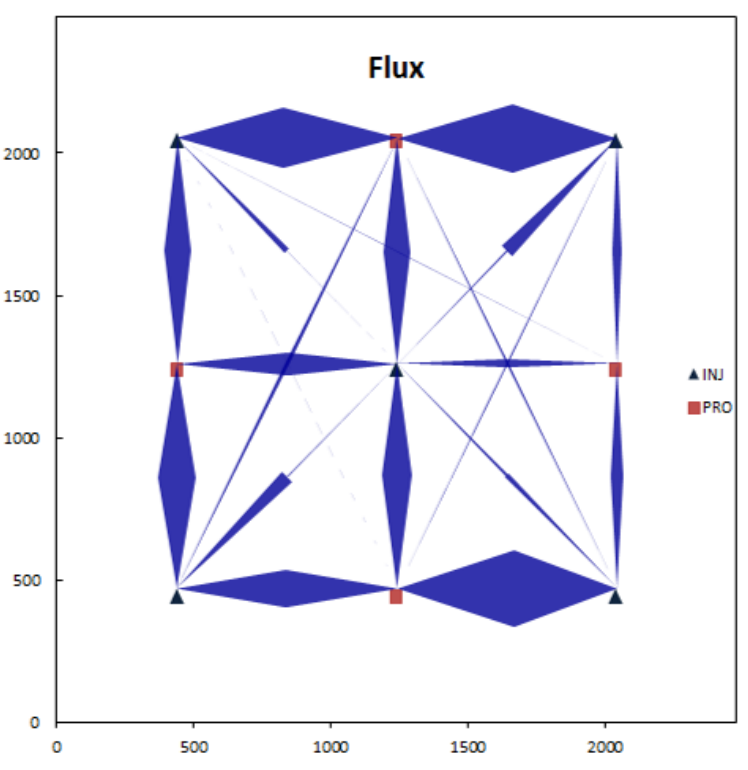

(b) Well allocation factor.

Figure 4.11: Model parameters for omni-direction permeability trend.

and 4.14. In addition to symmetry of the conductance values, values are almost equal from injector to injector. Like other examples in this chapter, there is a strong connection between injectors and adjacent producers. Injector-injector conductance values are not plotted in Figure 4.13 but their 


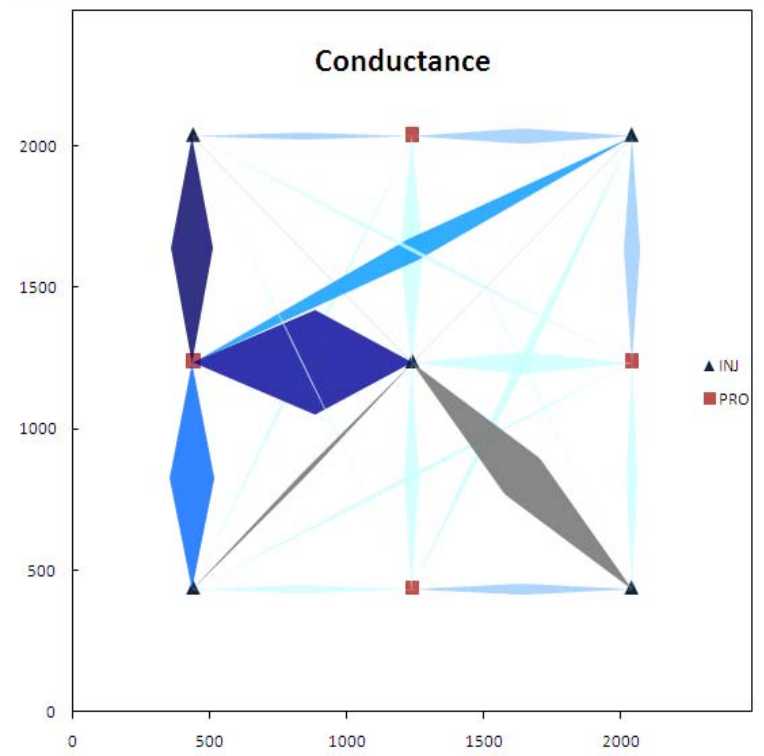

(a) Conductance values.

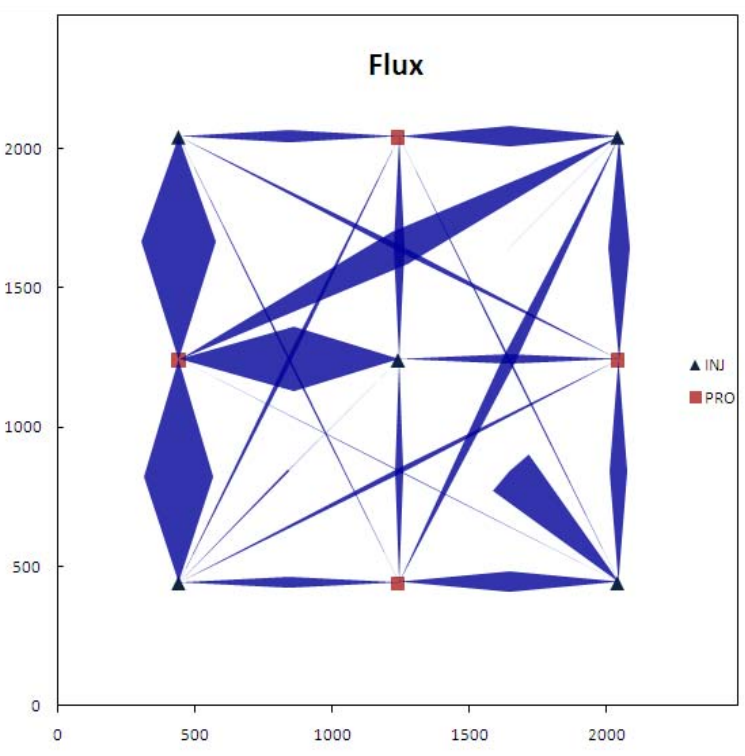

(b) Well allocation factor.

Figure 4.12: Model parameters for a high permeable channel with injector-injector interaction.

influence is shown in 4.14 where, there are more injector-injector influences for injectors at the corners.

The approach was also tested for a heterogeneous system, where the same number of wells and injection history has been applied. The permeability distribution is shown in Figure 4.15, where a NW-SE permeability trend is noticeable. The conductance values (Figure 4.16) and WAFs (Figure 4.17) agree with the direction of permeability trend, and stronger connections are obtained in this direction.

For a large number of wells, considering injector-injector interaction increases the variables of the system, because injector-injector connections are added to the injector-producer connections. However, this modification improves the result and gives conductance values that agree with properties of the domain. 


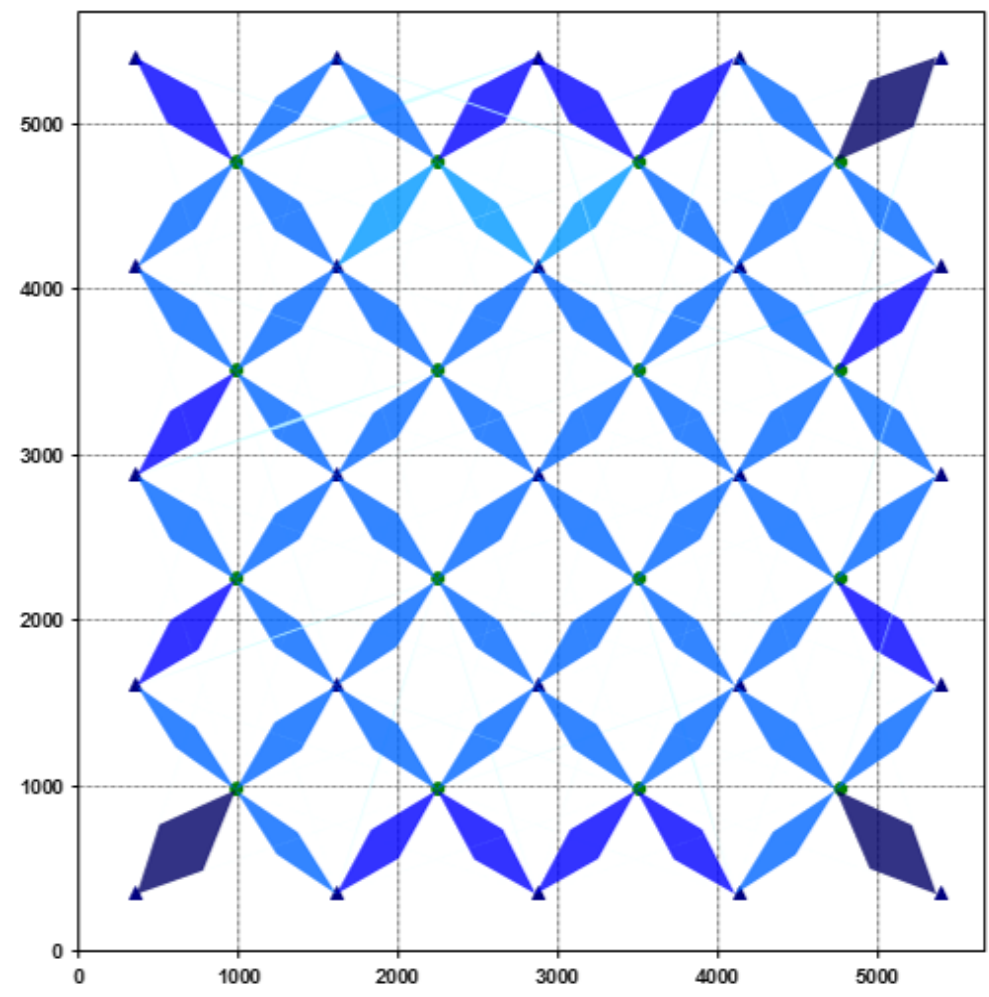

Figure 4.13: Conductance values for the nhomogeneous $16 \times 25$ Synfield.

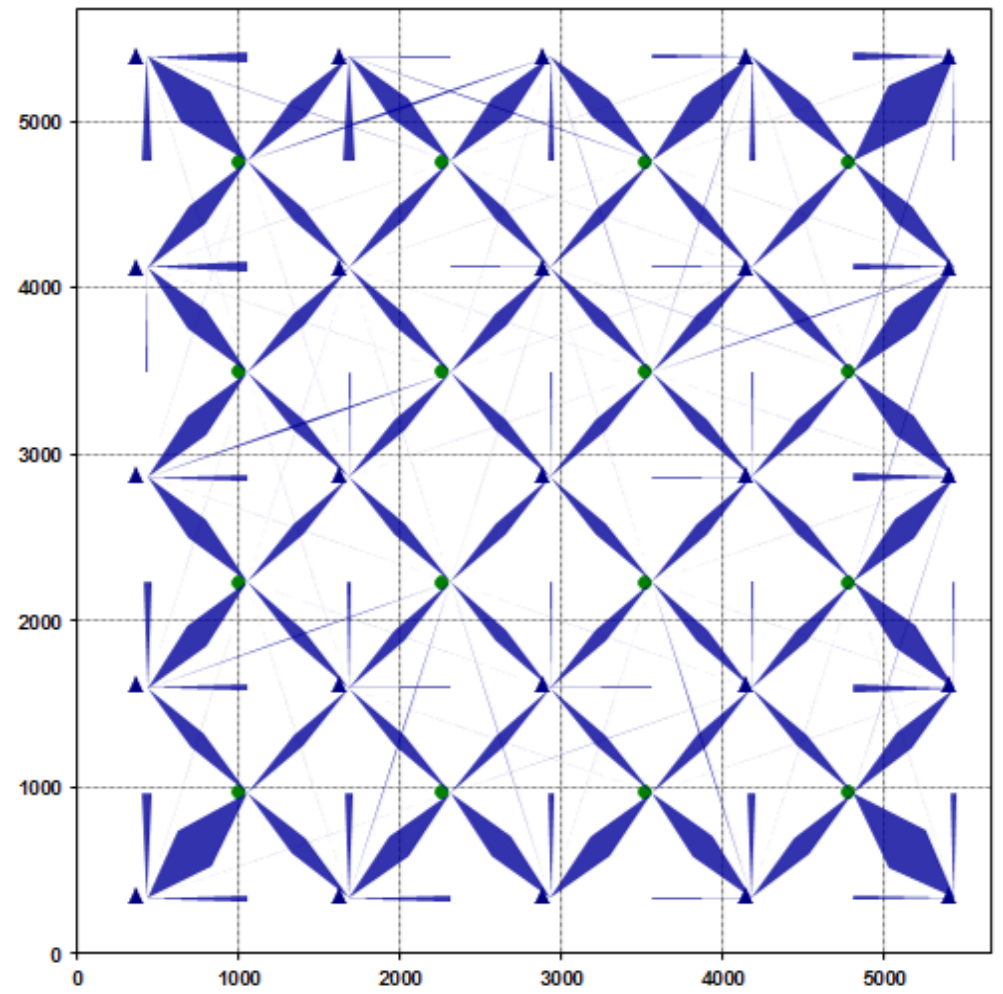

Figure 4.14: Well allocation factor for the homogeneous $16 \times 25$ Synfield. 


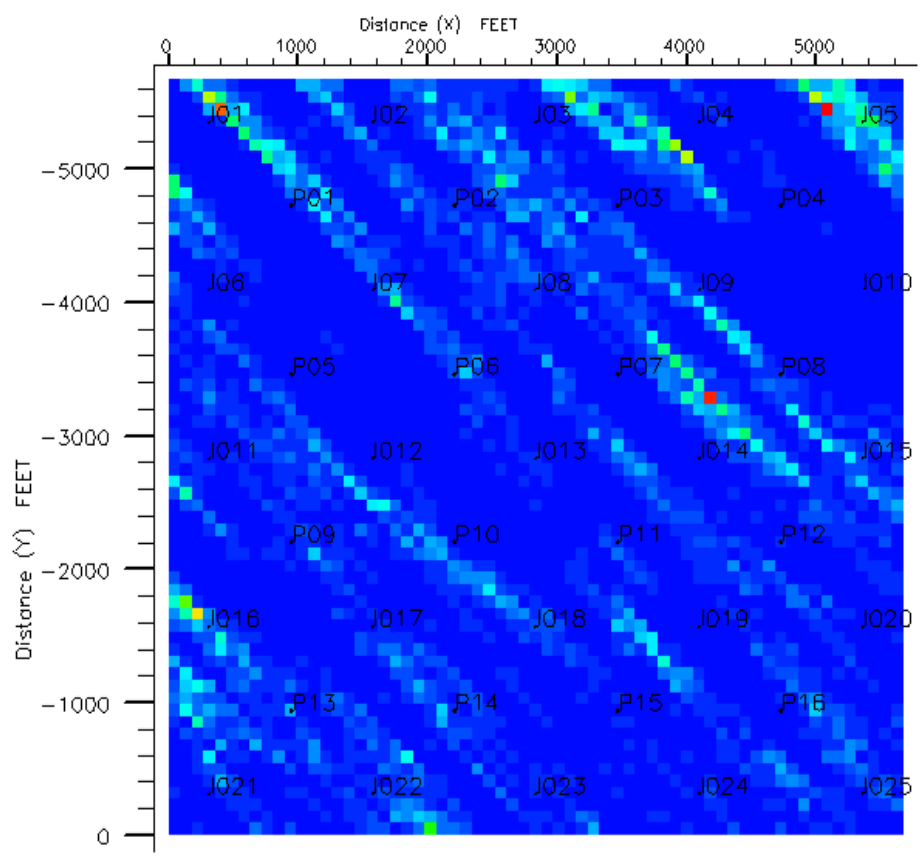

PermX (MDARCY)

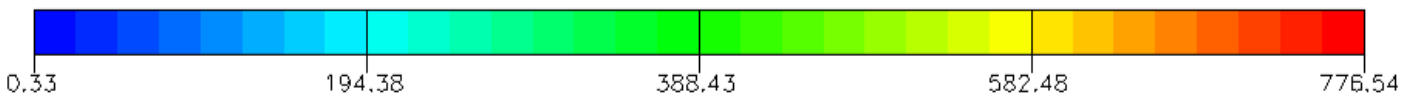

Figure 4.15: NW-SE direction permeability trend in $16 \times 25$ Synfield

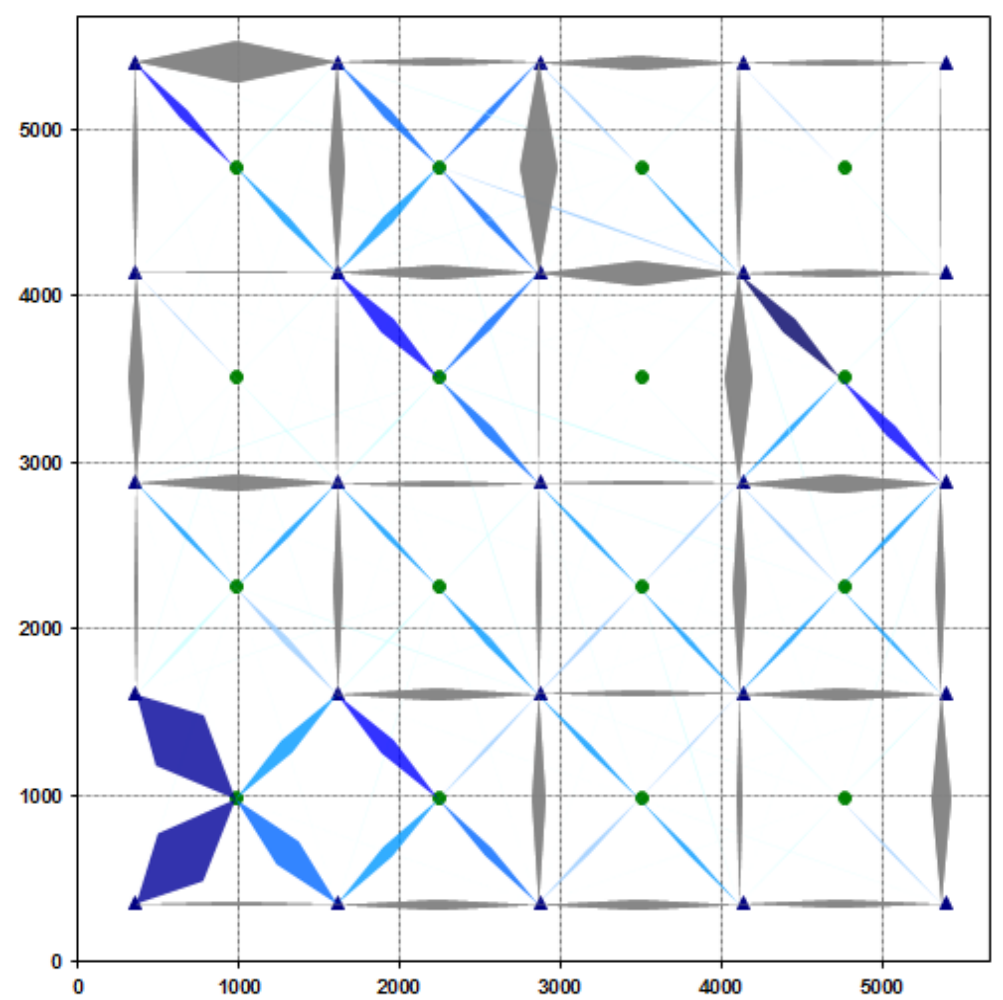

Figure 4.16: Conductance values for NW-SE direction permeability trend in the $16 \times 25$ Synfield. 


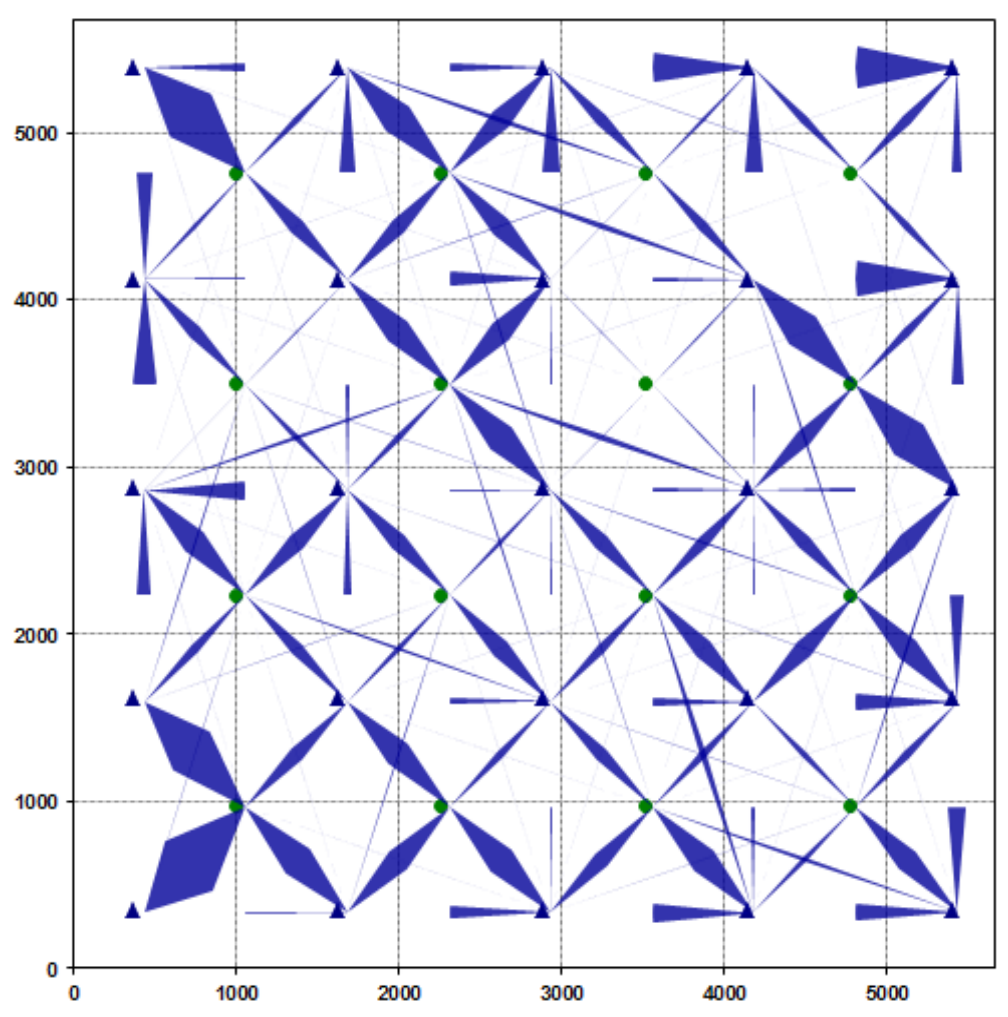

Figure 4.17: Well allocation factor for NW-SE direction permeability trend in the $16 \times 25$ Synfield.

\subsection{Conclusion}

Well allocation factors obtained from streamline simulation and tracer tests are different from what is obtained from connectivity models. Now the question is: do the allocation values obtained from streamline simulation have different meaning from connectivity models, or are they effectively the same and just a matter of using different methods?

One assumption is that rate fluctuations change the pressure field, and there are intervals in which nonadjacent wells may be connected. In addition, fluctuation in the injection rate of one injector may affect the interaction between other injectors and producers. This means that producers are sensitive to fluctuations in distant injectors. Since there isn't a direct connection between injection wells, this effect may be accounted for by an effect from the distant injector to the producer. However, in a streamline model, the pressure field around the injectors reacts to injection 
rate fluctuations and may be reflected by a change in the number or path of streamlines between injectors and producers.

In this section, this interaction was modeled by considering a bond between injectors. The first impact of this modification is an increase in adjacent injector-producer influence, and as a result a decreases in the influence of non-adjacent wells. This behavior agrees with the result of streamline simulation and tracer tests. In addition, obtained conductance values and WAFs represent the domain better than a model in which injector-injector connections are ignored. For example, more uniform values are obtained in cases of a homogeneous system. Finally, this modification gives more details about the reservoir features and makes it easier to infer such reservoir properties. 


\section{Chapter 5}

\section{Application to Field Data}

The network model technique was applied to the northwestern region of a reservoir provided by an operating company for which daily injection and production data were available for 96 producers and 66 injectors over 900 days. The problem was broken into smaller problems, since smaller ones have fewer unknowns and are easier to handle and because the company was mainly interested in the region selected. The following technical criteria were considered to determine the boundary of the region:

1. Total injection rate should be higher than total production rate for the region.

2. All high-rate wells should be included and any off-center low-rate injectors/producers may be excluded

3. Some portion of the reservoir should have a boundary

Using these criteria means that the inside injectors are the main sources of influence, and any boundary injectors should not significantly influence producers inside the region. The resulting region included 28 producers and 22 injectors. Fig. 5.1 shows the boundary of the region and well locations.

Knowing boundary and well positions and implementing Delaunay triangulation results in the Voronoi tiles depicted in Fig. 5.2. There are 49 Voronoi tiles for the 49 wells in the region. The number of tiles is less than the sum of the injectors and producers, since one producer was converted to an injector, and one tile was assigned to account for this change. To calculate the node volumes, each tile area was multiplied by an assumed reservoir thickness at each well.

Once node volumes were determined, connected volumes needed to be determined. In other words, one has to find out which producers are influenced by which injectors. This is done by assigning a radius of influence for every injector and connecting those producers that fall within 


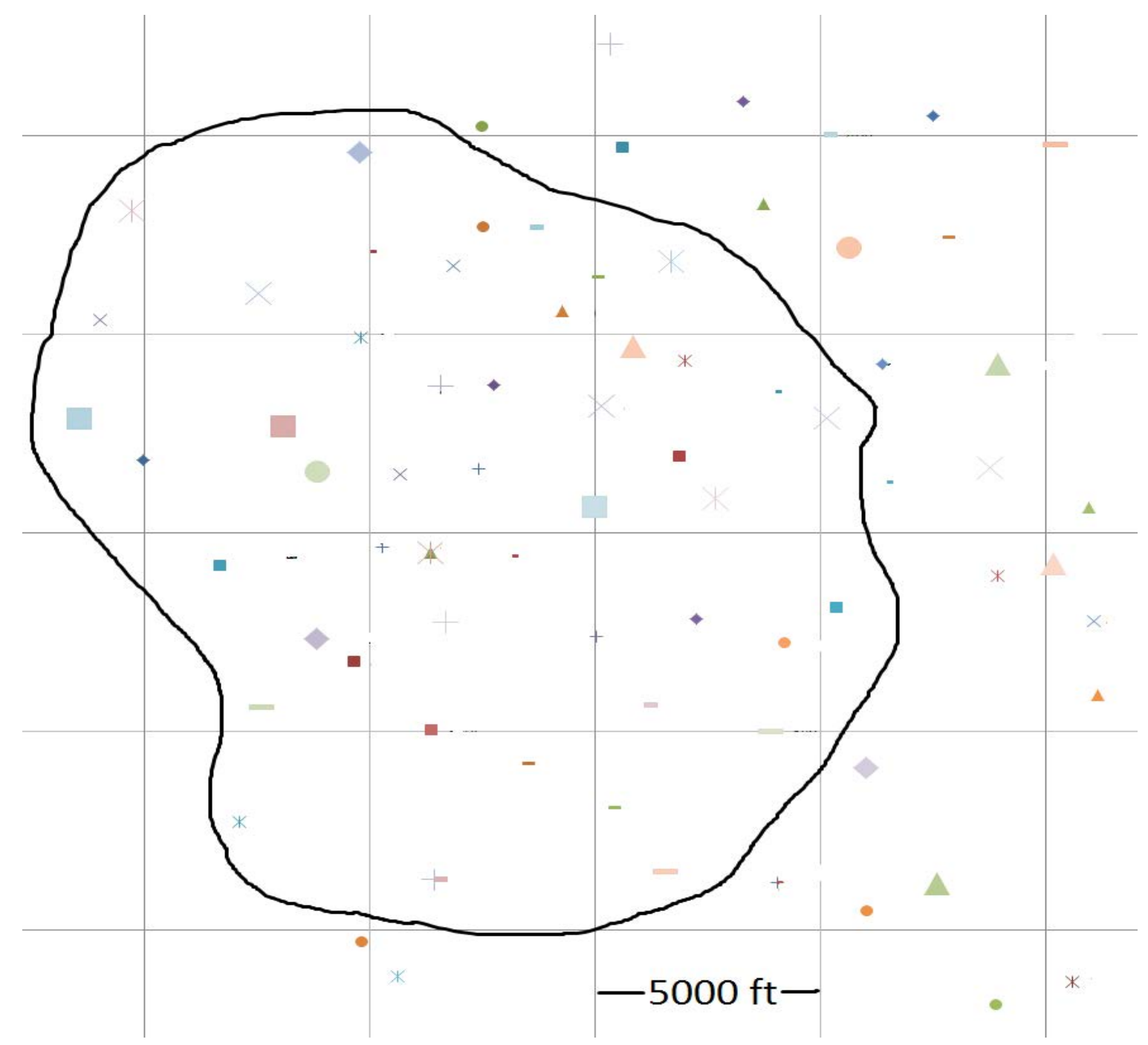

Figure 5.1: The study region in which, only the wells inside are studied. Injectors are shown by large and producers by small icons.

that radius. Sensitivity of the match to radius of influence is investigated in Fig. 5.3. Fig. 5.3 shows that increasing radius of investigation from $4000 \mathrm{ft}$ to $8000 \mathrm{ft}$ decreases the difference between observed and modeled production by a cumulative value of $15 \%$. This was considered to be a relatively minor influence, and 8000- $f t$ value was chosen, since distant injector/producers pairs needed to be considered, and the $8000 \mathrm{ft}$ results in less than 250 connections, a value that can be handled by the chosen optimization method.

Before showing history match results and conductance values, it is worth mentioning some of the difficulties associated with applying the method to real data as opposed to synthetic data. 


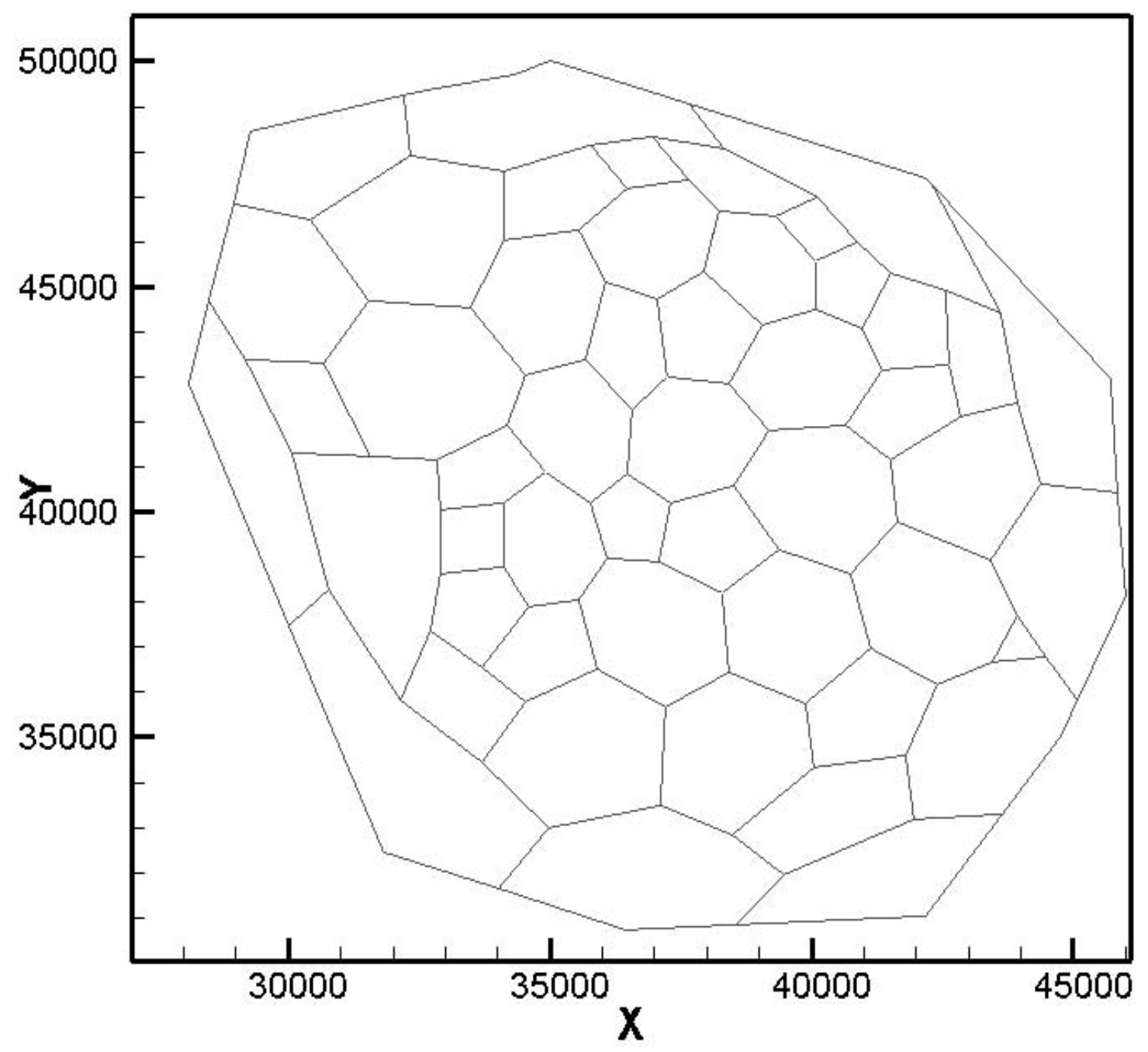

Figure 5.2: Voronoi tiles assigned to the wells inside the study region

\subsection{Open Boundary}

While the left side of the study region coincides with a reservoir boundary, and it can be assumed there is no flow across that boundary, the right side communicates with the rest of the reservoir and surrounding wells.

Fig. 5.4 shows the normalized (relative to the mean injection and production rates) total injection and production rates plus the average measured bottomhole pressures of the producers in the region. For most of the study period, total injection exceeds total production. Therefore, one can assume that fluid flows from inside to outside the region. To handle this outward flow, all injec- 


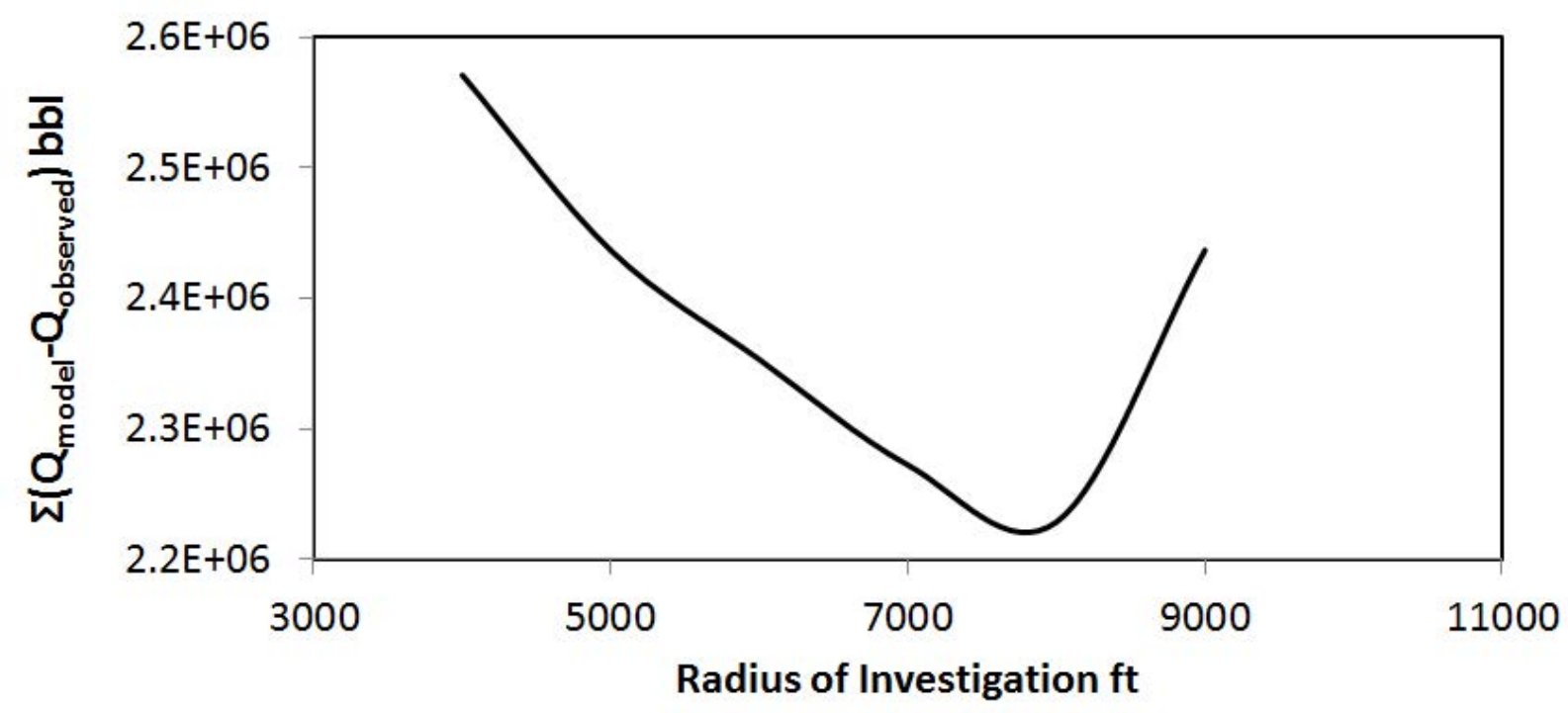

Figure 5.3: Sensitivity analysis on the radius of influence of injectors

tors are connected to a constant low-pressure sink. In other words, a constant-pressure boundary condition is defined, and all the injectors are connected to the sink through a bond, which has an unknown conductance value. Therefore, conductance values that connect injectors to this constant pressure boundary need to be estimated in addition to the injector-producer conductance value. Once these values are found, one can estimate what percentage of injection leaves through the boundary.

\subsection{Converting a producer to an injector}

When producers are converted to injectors, the number of injectors and producers changes, and the set of conductance values that connects the converted producer to injectors is no longer applicable. Instead, a new set of conductance values needs to be defined that connects the new injector to producers. If producer $J$ is converted to an injector at time $t$, the same node volume can be used. But after time $t$, all conductance values that connect producer $J$ to injectors are set to zero, and conductance values from the new injector to producers that were set to 0 before time $t$ become new optimization parameters. This approach is also applicable when injector-injector interaction is considered in the model. 


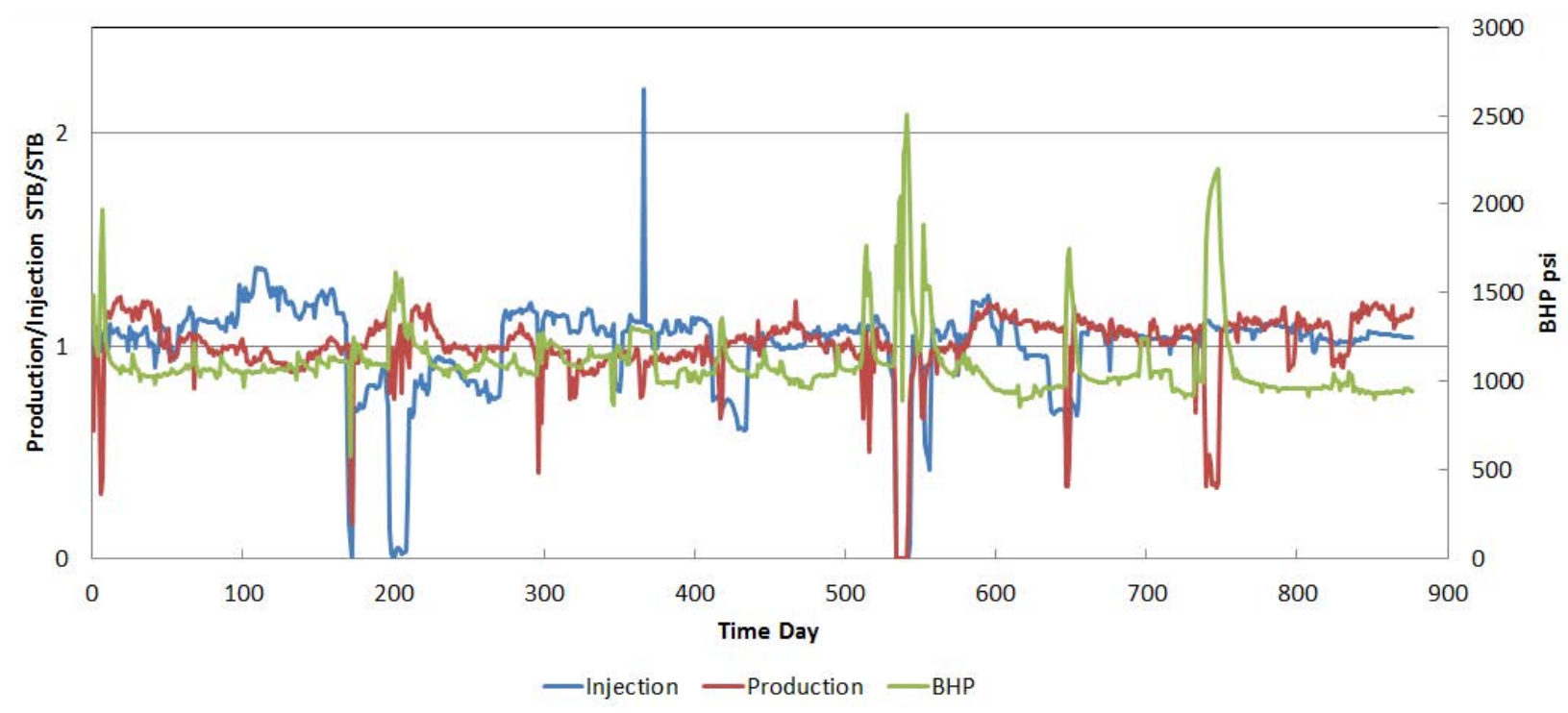

Figure 5.4: Production and injection rates and bottomhole pressure for 900 days study period

\subsection{Change in productivity}

As was discussed in section 3.1, well index and the difference between node-volume pressure and bottom-hole pressure determine well rate. Node volume pressure usually is the shut-in pressure or average reservoir pressure when shut-in pressure is not available. If bottomhole pressure is known, productivity is calculated using rate and node volume pressure. When BHP is not available, the well index value is assigned using the well average production rate and a correlation that relates this average to the well index. Such a correlation is shown in Fig. 5.5, where each point represents the well index for each well at times where pressure data are known. At least two data points should be available to generate what is shown in Fig. 5.5.

What is assigned to the producers is an average well index, since it is based on the average production rate. Damage and stimulation may change well productivity during the study period. A model with one average well index may not match observed production in these cases. To handle variable productivity, well index values at each time step can be calculated or be external inputs to the model. 


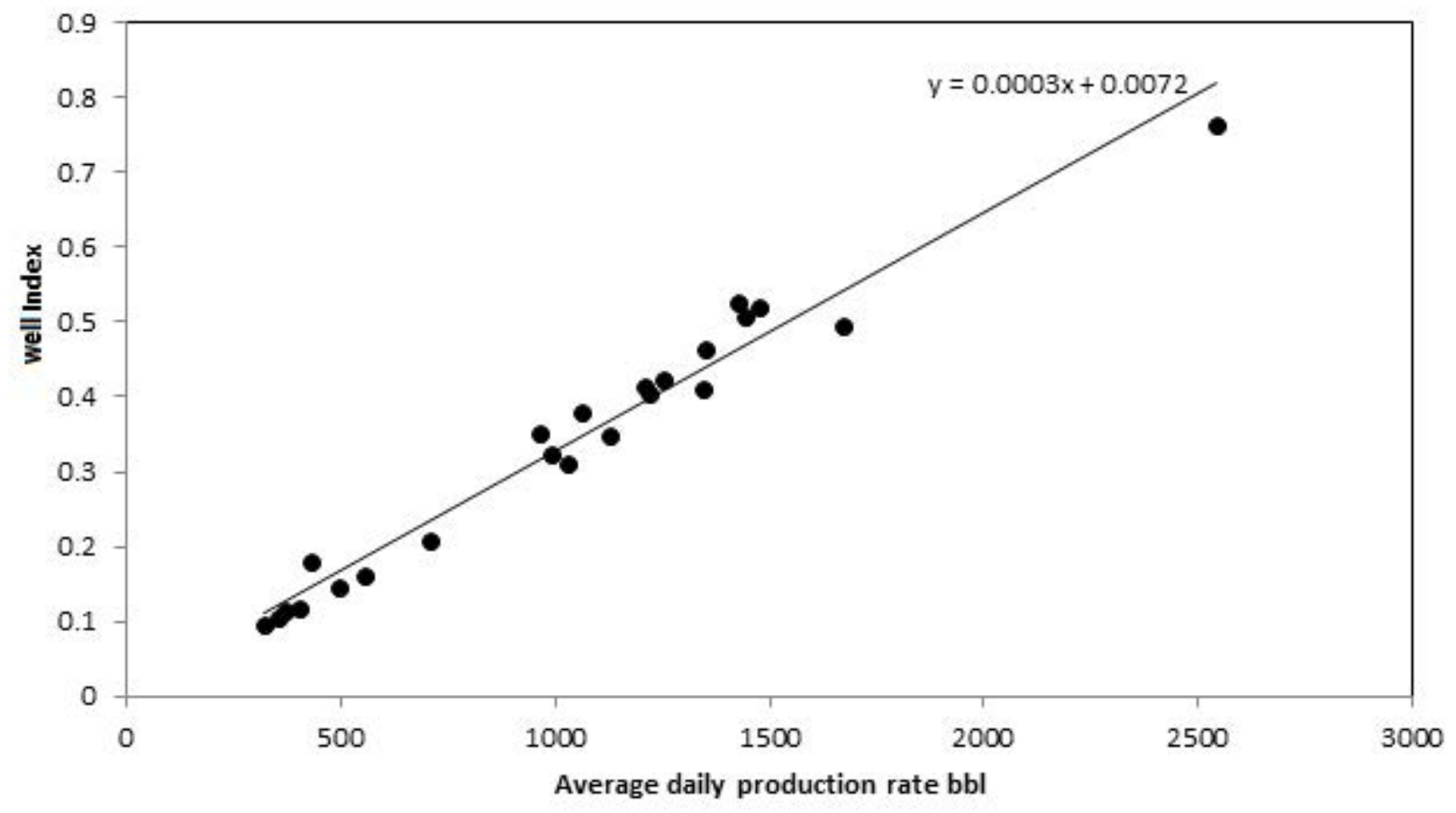

Figure 5.5: Productivity index vs average daily production rate

\subsection{Part-time injection/production}

Part-time injection or production conveys the fact that the well is partially opened during the intervals of data collecting. For example, a well may produce half a day and then be shut-in for the next half if the interval of data-collecting is one day. This issue is ignored for injectors, because the amount of injection into the node volume is the primary influence variable. For producers, production time should be included in the model, since the longer the producer is on, the more fluid it produces. To handle part-time production, the ratio of time-on production to the data collection interval time is calculated. A 0 value means no production occurs during that interval, and a value of 1 means production occured throughout the interval. Values between 0 and 1 show part-time production. This ratio is multiplied by the productivity in the well model equation:

$$
q_{s c}=-R_{p} J_{w}\left(p_{J}-p_{w f}\right)
$$

The $R_{p}$ term is the ratio of production time to the interval of data-collecting time. When the value of $R_{p}$ changes from 0 to 1 , production varies from 0 to $J_{w}\left(p_{J}-p_{w f}\right)$. 


\subsection{Results}

In this section, conductance values are estimated by matching model response with observed production rates. Like any history matching problem, uncertainty associated with input data make the matches non-unique, because different sets of uncertain reservoir parameters can match observed production. Although the network model has reduced the number of variables, uncertainty still plays a role in conductance value estimation. Even individual well production values may not be certain and have some inherent error compared to total field production. Sayarpour et al. (2011) mentions the allocation issue as a reason for this discrepency and they assume a $10 \%$ error.

In the network model, node volume is one of the main sources of uncertainty. Both area and thicknesses of the node may vary, depending on the method and assumptions that are implemented. Fig. 5.6 and Fig. 5.7 show allocation factors for two different sets of node volumes. In Fig. 5.6, tile areas are averaged, and constant values for the assumed reservoir thickness at wells are used to calculate the node volumes. In Fig. 5.7, Voronoi tile areas are multiplied by an assumed net pay thickness at each well to obtain the node volumes. Thickness of the bonds shows the percentage of flux that goes from injector to producers (similar to an allocation factor), and the darkness of the bonds show the amount of flux.

Bubbles in Fig. 5.8 and Fig. 5.9 show injection that leaves the boundary of the region. The larger the bubble, the larger the amount of injection that leaves the boundary. Fig. 5.10 and Fig. 5.11 show the same type of graphs, but this time injector-injector interactions are considered in the model. Thin bonds in Fig. 5.11 represent the injector-injector connections.

The way injector 36 in the north-east of the study region communicates with surrounding injectors and producers is a good example to show the effect of considering the injector-injector interactions. In Fig. 5.6 and 5.7 injector 36 is connected to the producer 16 through a direct connection (flux connected). In the model in which injector-injector interactions are included, injector 36 is connected to injector 35 (Fig. 5.11), and injector 35 is connected to producer 16 (Fig. 5.10). In other words, injector 36 is indirectly connected to producer 16 through injector 35 (pressure con- 
nected). On the other hand, connection between injector 40 on the west with producer 1 appears to be a direct connection. This connection is noticeable in all the well allocation plots (Figures 5.6, 5.7, and 5.10). If tracer is injected in injector 40, it should be seen in producer 1.

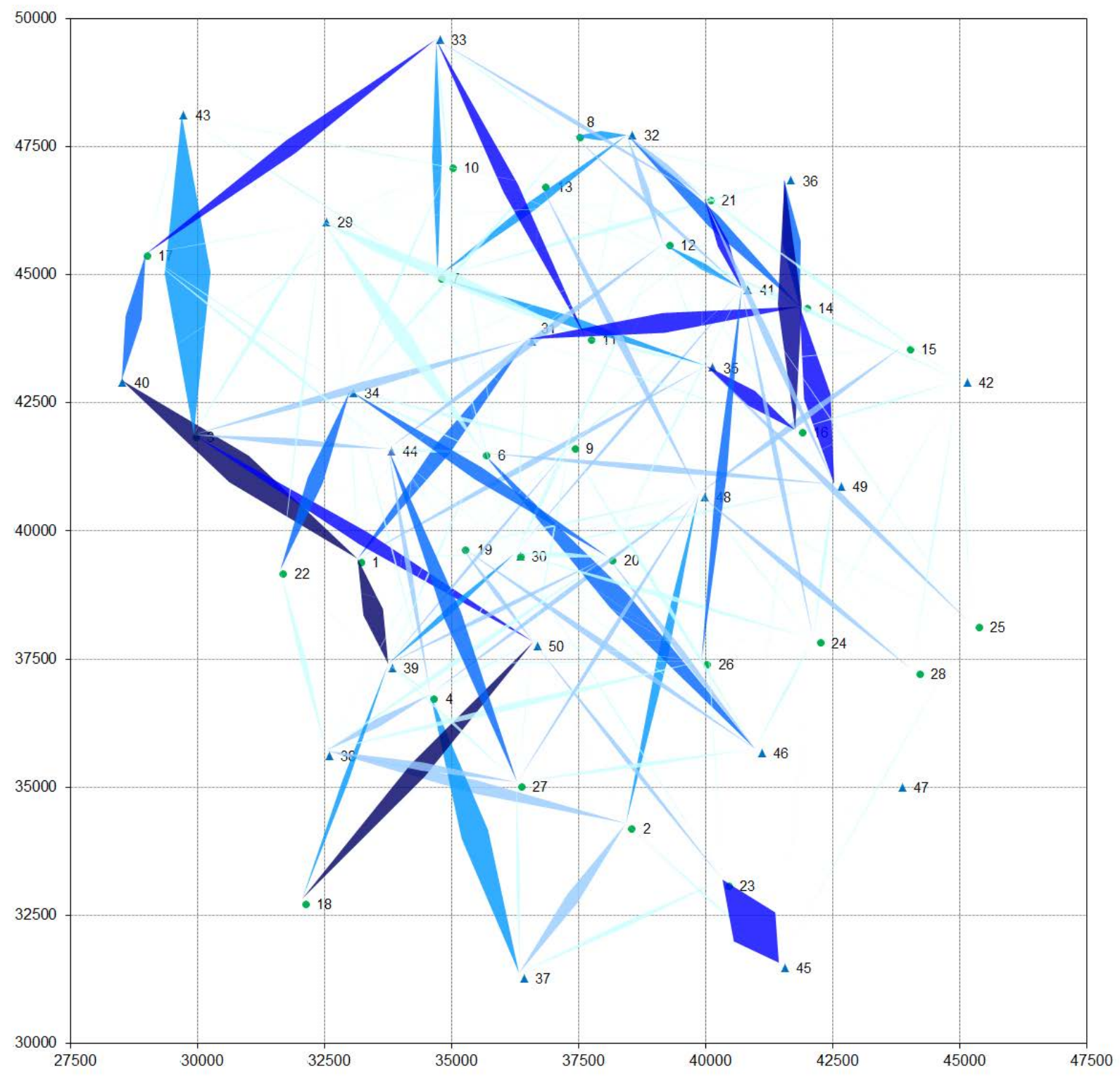

Figure 5.6: Well allocation factor for equal thickness and equal tile areas 


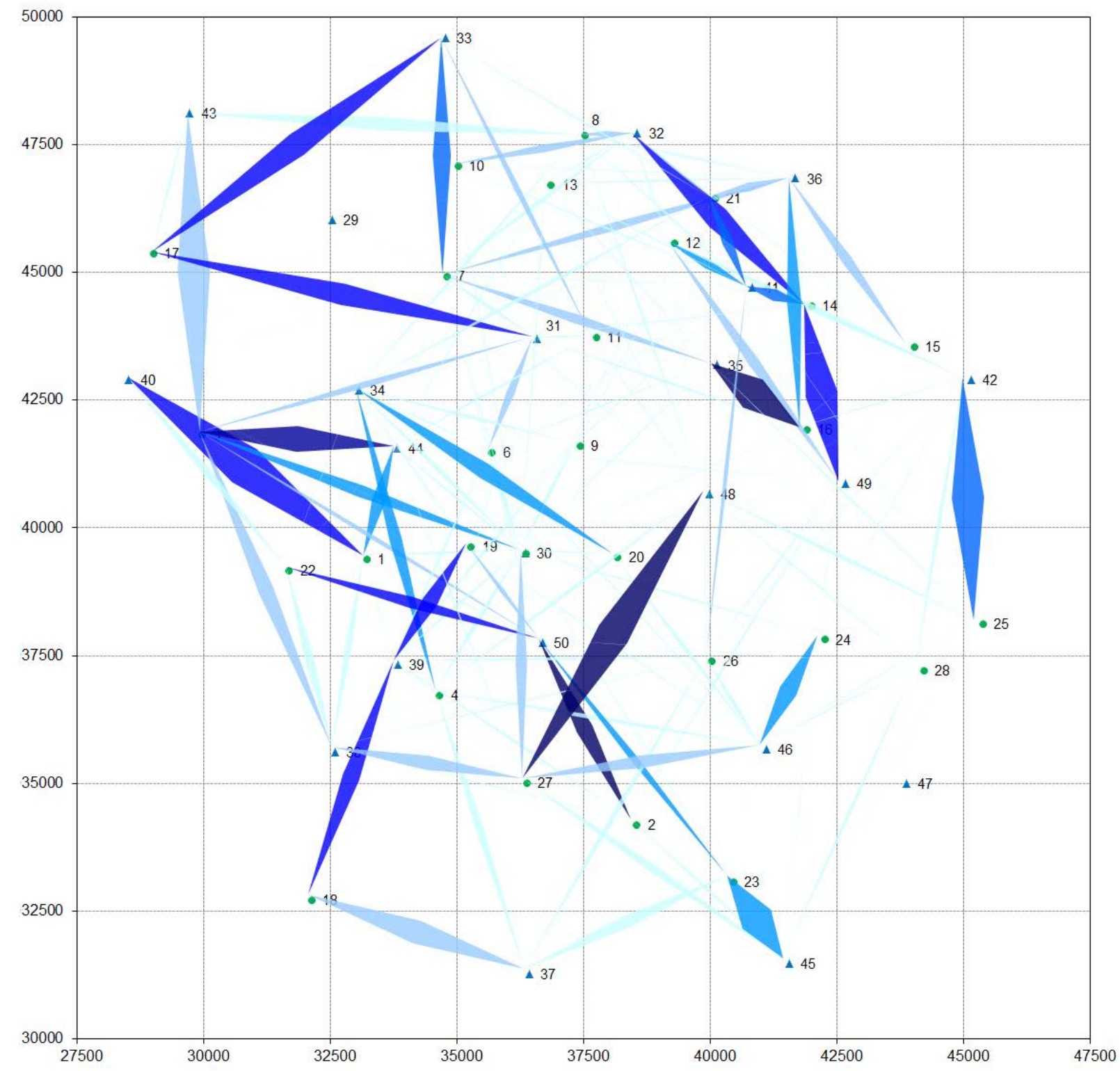

Figure 5.7: Well allocation factor for pay thickness and Voronoi tile areas

\subsection{Conclusions}

Uncertainty in the network model comes from node volume, fluid compressibility, radius of influence, and well indices. In previous chapters it was mentioned that it is safer to use a large enough radius of influence to include all affected producers. It was also mentioned that in the case of using incorrect well indices, the computed conductance values adjust to respond to the assumed well 


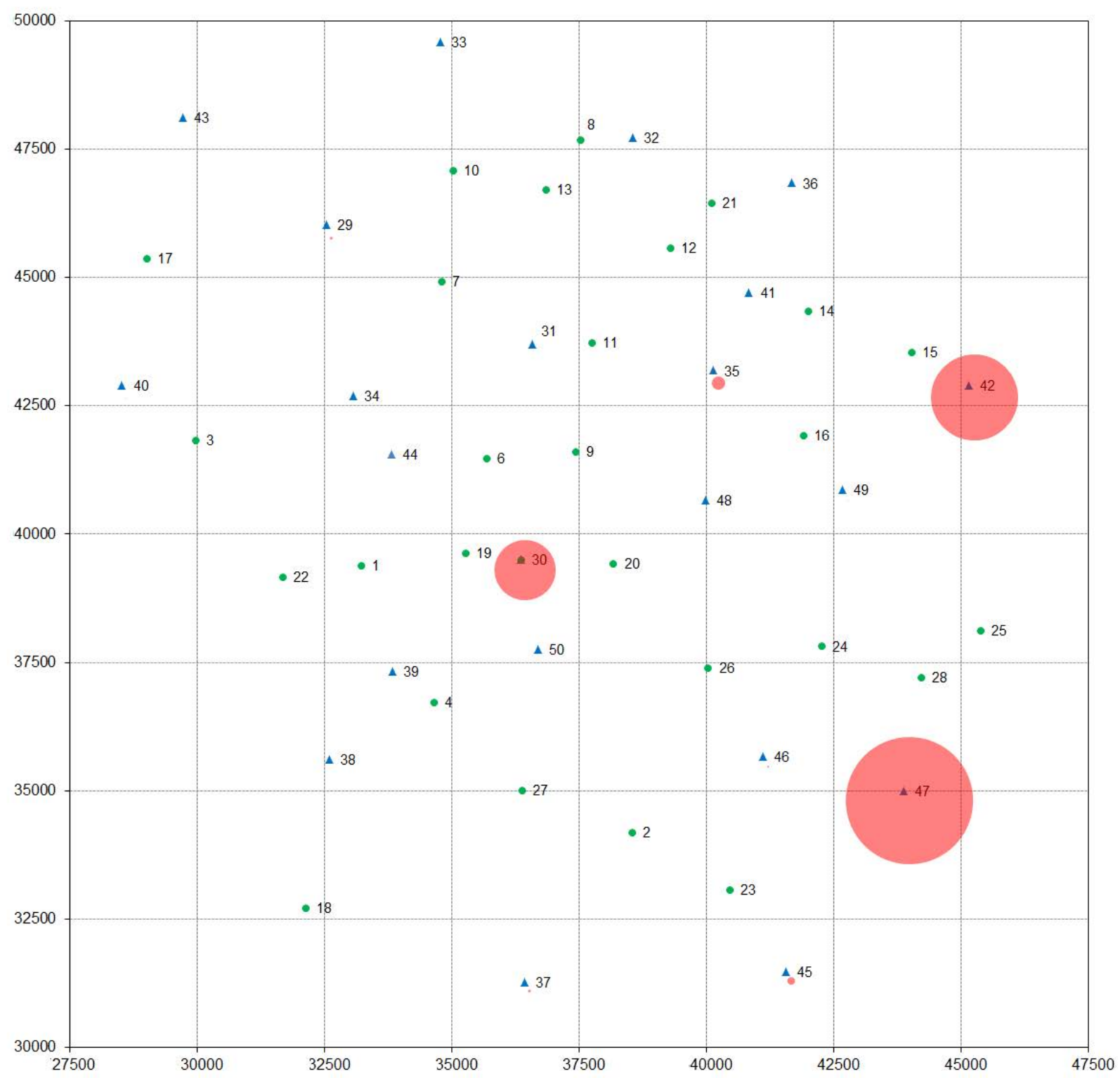

Figure 5.8: Out of boundary flow for equal thickness and equal tile areas

indices. Fluid compressibility is also somewhat known, and rough estimates of the saturation of each phase provide an average fluid saturation for each node. The same applies for node volume, which means the model is more reliable if the sum of the identical estimates of each node volume is approximately equal to the actual total reservoir volume. A rough estimation of reservoir volume would be the sum of the net pay thicknesses at each well times the Voronoi tile areas. If net pay data are not available (as in this case), using equal thickness and equal tile areas would be a 


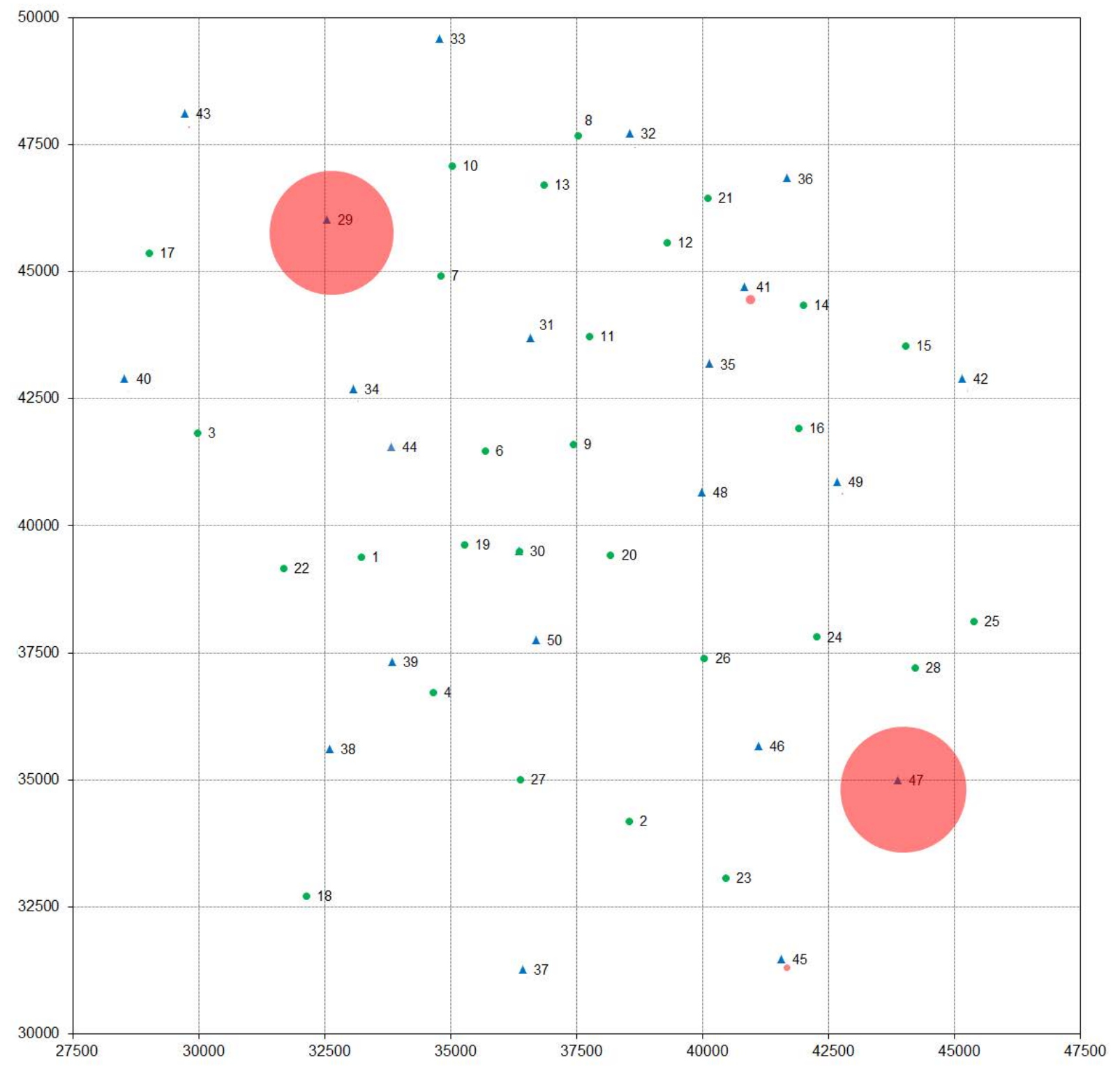

Figure 5.9: Out of boundary flow for pay thickness and Voronoi tile areas

reasonable first estimate. One can also use a correction factor to adjust the reservoir volume in this case. This factor can be set up as a match parameter and will be multiplied by all node volumes.

In addition, the influence of each of the above factors on production should be compared with errors in production rates. That is why Sayarpour et al. (2009) emphasize that data with noticeable pulses in injectors and producers are better candidates for using these methods. Again, production rate changes as a result of these pulses should be more than errors in the production data. 


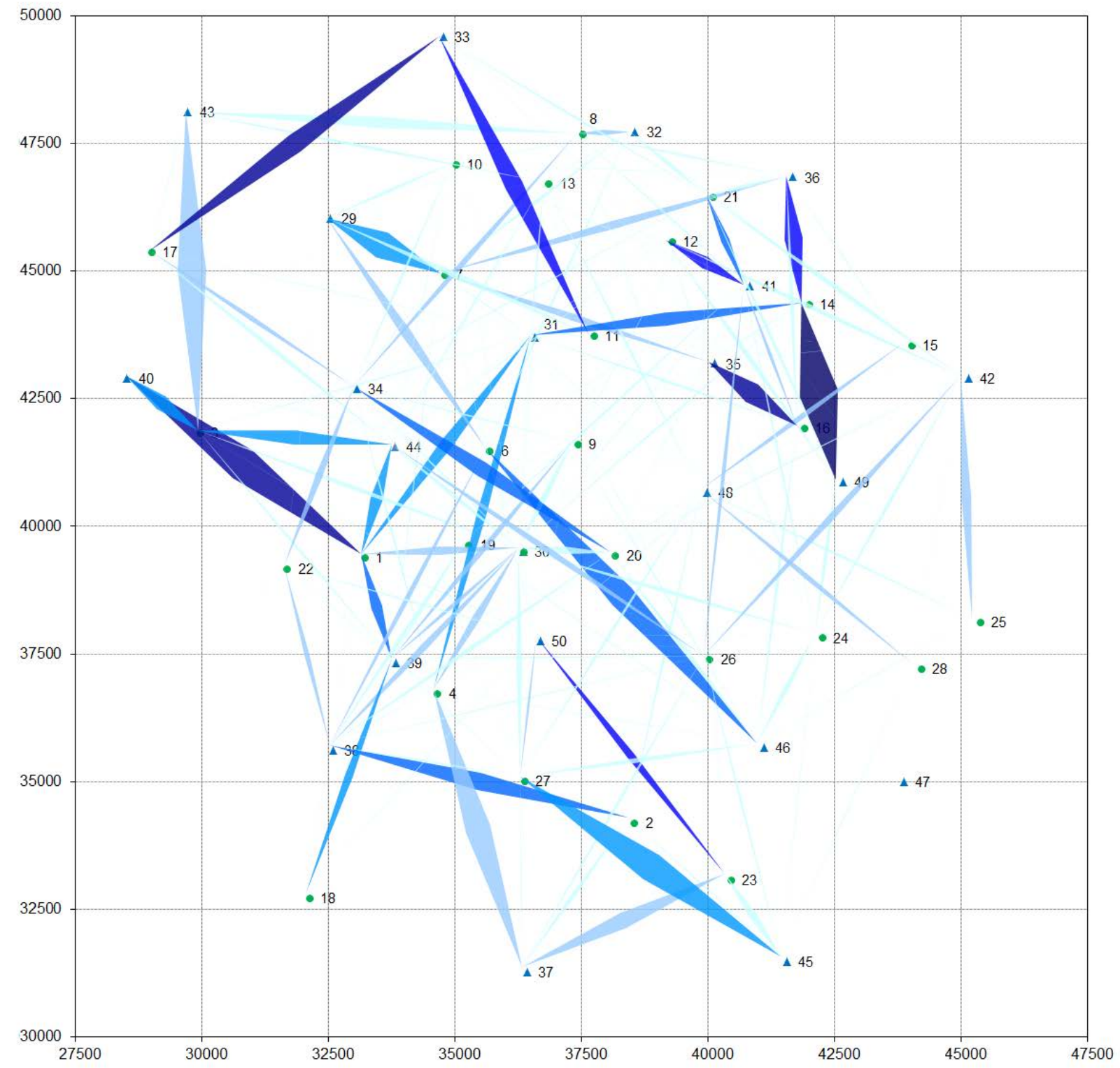

Figure 5.10: Well allocation factor for equal thickness and equal tile areas with injector-injector connection

For cases like the one studied here, where there are no noticeable pulses and there are many equally probable solutions, the question becomes: which is the more reliable solution? Would adding more data really help? The answer is that more reliable data should lead to a better decision. But there are only a few ways to test the decision, since these methods are usually applied to fields where detailed reservoir models are not available. 


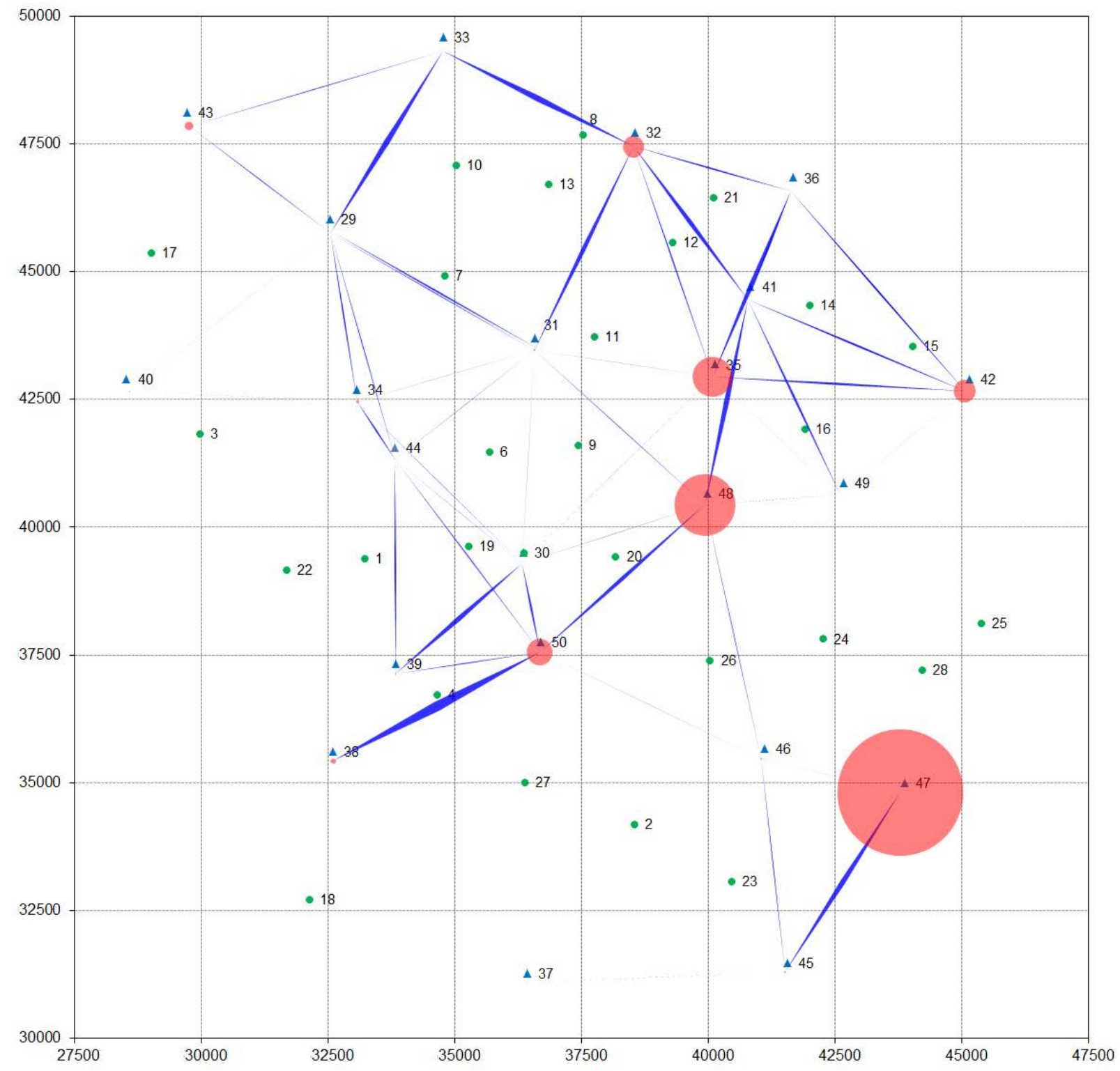

Figure 5.11: Out of boundary flux and injector-injector allocation factor for equal thickness and equal tile areas with injector-injector connection

For now, we suggest comparing the conductance values and outflowing injectors and downgrading the results that do not make physical sense. For example, comparing results for the case where node volumes are calculated by Voronoi tiles and the assumed net pay map ( Fig. 5.7 and Fig. 5.9) and the case with equal area and thickness values (Fig. 5.6 and Fig. 5.8), one notices that connections for wells near the boundaries are not as strong in the Voronoi tile/net pay map case, there is 
more uniformity, there are fewer crossing connections, and there are stronger northwest-southeast connections, which could then be compared to any direction of correlated permeability. In addition, in comparing the amount of flow out of the region, the Voronoi tile/net pay results (Fig. 5.9) appear to make more sense than the equal-pay results (Fig. 5.8), since outflowing injectors are closer to the boundary in Fig. 5.9 than Fig. 5.8. 


\section{Chapter 6}

\section{Two Phase Modeling}

Improving waterflooding efficiency is one of the main challenges in mature waterflooded fields. The single-phase network model approach matches total production and finds principal features of the reservoir similar to correlation-based models; however, to further improve the flood an oil production model is needed. A two-phase network model approach is proposed, which uses conventional two-phase flow equations. In this model, in addition to conductance of the bonds, the ability of fluids to flow in the presence of each other through the bonds should be determined. This is analogous to the concept of relative permeability. In this chapter a method to determine these two-phase flow parameters is presented.

\subsection{Two-Phase Flow Equations}

Neglecting capillary pressure, flow equations for oil and water may be written as:

$$
\begin{gathered}
\sum_{J=1}^{N_{j}} \beta_{c} \frac{k_{I J} k_{r o}}{B_{o} \mu_{o}} \nabla p_{I J} A_{c s I J}+Q_{o}{ }^{s c}=\frac{V_{b}}{\alpha_{c}} \frac{\partial}{\partial t}\left(\frac{S_{o} \phi}{B_{o}}\right) \\
\sum_{J=1}^{N_{j}} \beta_{c} \frac{k_{I J} k_{r w}}{B_{w} \mu_{w}} \nabla p_{I J} A_{c s I J}+Q_{w}{ }^{s c}=\frac{V_{b}}{\alpha_{c}} \frac{\partial}{\partial t}\left(\frac{S_{w} \phi}{B_{w}}\right) \\
\sum_{J=1}^{N j} \beta_{c}\left(\frac{1}{B_{o}{ }^{n} \mu_{o}{ }^{n}}\right)\left(\frac{A_{c s I J}}{L_{I J}}\right) k_{I J} k_{r o}\left(P_{J}^{n+1}-P_{I}^{n+1}\right)+Q_{o}{ }^{s c}=\frac{V_{b} \phi}{\alpha_{c}}\left[\frac{-1}{B_{o}{ }^{n+1}} \frac{\partial S_{w}}{\partial t}+\left(1-S_{w}\right) \frac{c}{B_{o}{ }^{0}} \frac{\partial P}{\partial t}\right] \\
\sum_{J=1}^{N j} \beta_{c}\left(\frac{1}{B_{w}{ }^{n} \mu_{w}{ }^{n}}\right)\left(\frac{A_{c s I J}}{L_{I J}}\right) k_{I J} k_{r w}\left(P_{J}^{n+1}-P_{I}^{n+1}\right)+Q_{w}{ }^{s c}=
\end{gathered}
$$

One can group the variables to reduce the complexity of the equations:

$$
\text { TRANS }_{\text {OIJ }}=\beta_{c}\left(\frac{1}{B_{o}{ }^{n} \mu_{o}{ }^{n}}\right)\left(\frac{k_{I J} A_{c S I J}}{L_{I J}}\right) k_{r o}
$$




$$
\begin{gathered}
T R A N S_{W I J}=\beta_{c}\left(\frac{1}{B_{w}{ }^{n} \mu_{w}{ }^{n}}\right)\left(\frac{k_{I J} A_{c S I J}}{L_{I J}}\right) k_{r w} \\
C O W=\frac{V_{b} \phi}{\alpha_{c} \Delta t} \frac{-1}{B_{o}{ }^{n+1}} \\
C O P=\frac{V_{b} \phi}{\alpha_{c} \Delta t}\left(1-S_{w}\right) \frac{c}{B_{o}{ }^{0}} \\
C W W=\frac{V_{b} \phi}{\alpha_{c} \Delta t} \frac{-1}{B_{w}{ }^{n+1}} \\
C W P=\frac{V_{b} \phi}{\alpha_{c} \Delta t} S_{w} \frac{c}{B_{w}{ }^{0}}
\end{gathered}
$$

Multiplying Eq. 6.4 by $A=\frac{B_{w}{ }^{n+1}}{B_{o}{ }^{n+1}}$ and adding Eq. 6.3 and Eq. 6.4 yields the pressure equation:

$$
\begin{aligned}
\sum_{J=1}^{N j}\left(\operatorname{TRANS}_{O I J}+\operatorname{TRANS}_{W I J}\right)\left(P_{J}^{n+1}-P_{I}^{n+1}\right)+Q_{o}{ }^{s c}+A Q_{w}{ }^{s c} & \\
& =\operatorname{COP}\left(P_{I}^{n+1}-P_{I}^{n}\right)+A C W P\left(P_{I}^{n+1}-P_{I}^{n}\right)
\end{aligned}
$$

Knowing pressure values, saturation may obtained from:

$$
S_{w}{ }^{n+1}=\frac{1}{C W W}\left[\sum_{J=1}^{N j}\left(\operatorname{TRANS} S_{W I J}\right)\left(P_{J}^{n+1}-P_{I}^{n+1}\right)-C W P\left(P_{I}^{n+1}-P_{I}^{n}\right)+Q_{w}{ }^{s c}\right]+S_{w}{ }^{n}
$$

A well model relates production to well bottomhole pressure and node volume pressure:

$$
\begin{gathered}
Q_{w}{ }^{s c}=-J_{w} f_{p}\left(P_{J}-P_{w f}\right) \\
Q_{o}{ }^{s c}=-J_{w}\left(1-f_{p}\right)\left(P_{J}-P_{w f}\right)
\end{gathered}
$$

The $k_{r w}$ and $k_{r o}$ terms in these equations are the network model water and oil relative permeability values at the node interfaces (bonds), and $f_{p}$ in the well model is the water fractional flow at 
the producer. The value of $k A_{c s}$ is determined from the single-phase network model; therefore, the network model relative conductivity at the node interfaces and the fractional flow values at each well are the only unknown parameters of this model that need to be determined. This chapter explains how these parameters are obtained using concepts from streamline fractional flow between injectors and producers.

\subsection{Streamlines}

In conventional streamline simulation, single-phase flow equations are solved to obtain a pressure field throughout the domain. After this pressure field has been determined, a velocity field is obtained based on the pressure field. Once the velocity field has been defined, one can trace streamline paths within a grid block. The method that is developed in this chapter is similar to mapping saturation values along streamlines and finding fractional flow at different distances from injectors. It is different from the conventional streamline simulation, since it does not need a geologic grid to trace the streamlines.

Instead of tracing streamlines on grid blocks from injectors to producers (Figure 6.1a), a large bond is defined between each injector-producer pair (Figure 6.1b). This bond shares volume fraction of the injector-producer pair that contribute to the flow. Knowing the volume of each bond, it is possible to relate each bond to at least one streamline, the streamline that passes through the center of the bond. In order to simplify the bond volume calculation, the bond is represented by a diamond shape as shown in Figure 6.1c. The volume of the bond is then a function of the distance between the injector and the producer, and the largest cross sectional area of the bond. In addition to simplicity, the velocity profile is closer to reality in a homogeneous system with this bond shape.

\subsection{Time of Flight}

Having an area open to flow along the bond and the flow rates through the bond, an incremental velocity along the bond can be obtained from: 


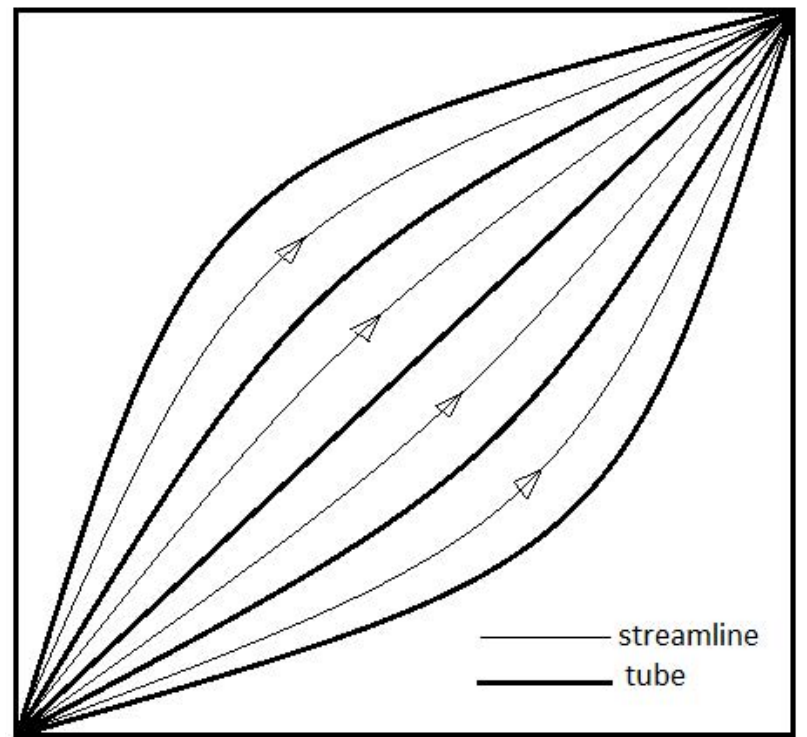

(a) Tubes and streamlines between an injector and a producer in a homogeneous medium.

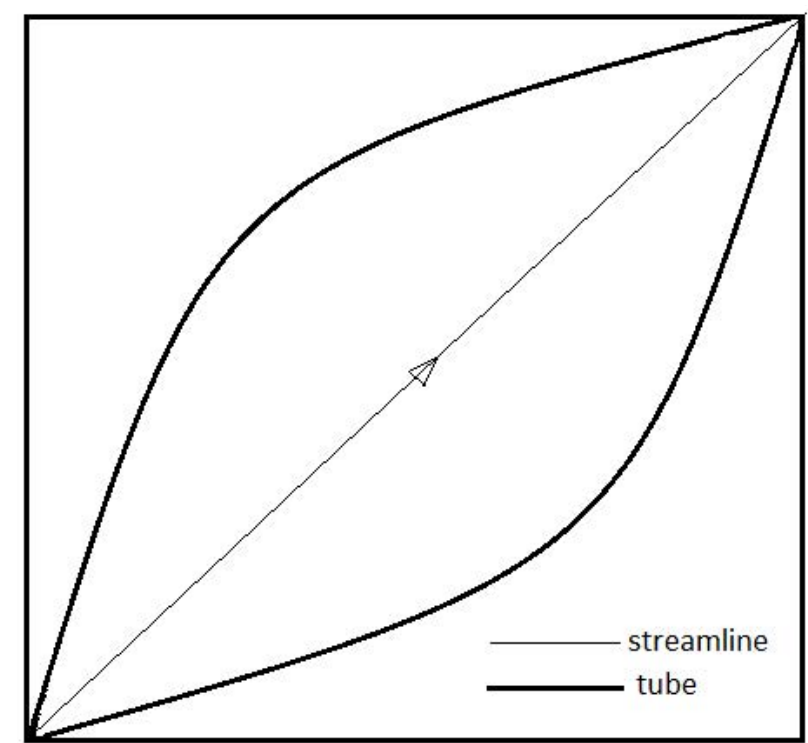

(b) Replacing bond with one large bond.

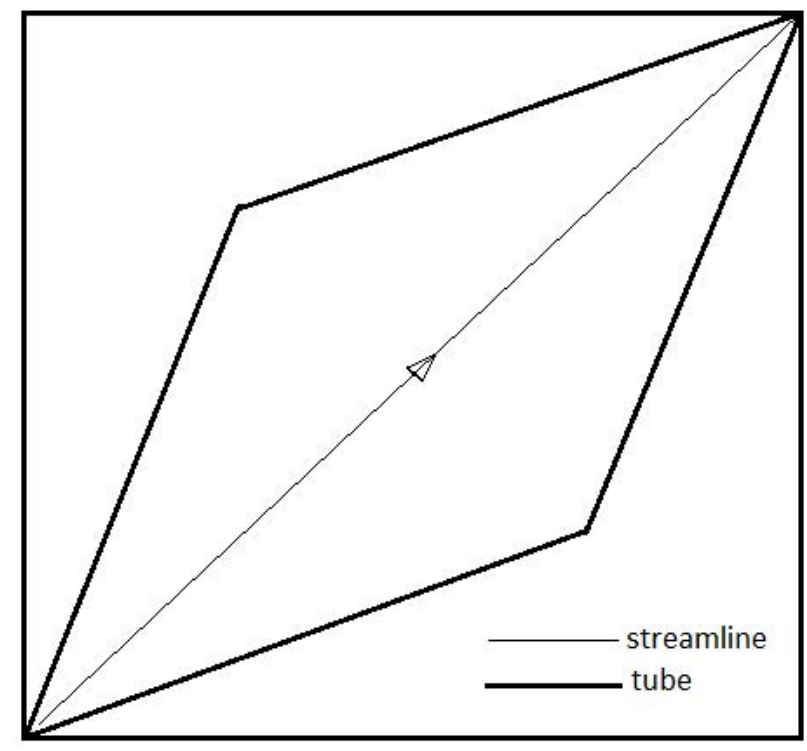

(c) The diamond shape tube.

Figure 6.1: Replacing bonds with a diamond shape bonds.

$$
u_{t}(s)=\frac{q}{A_{s}}
$$

$A_{S}$ is the area open to flow and is a function of the distance between wells and the largest area open to flow (Figure 6.2). $q$ is the flow between each injector-producer pair. Then the incremental 


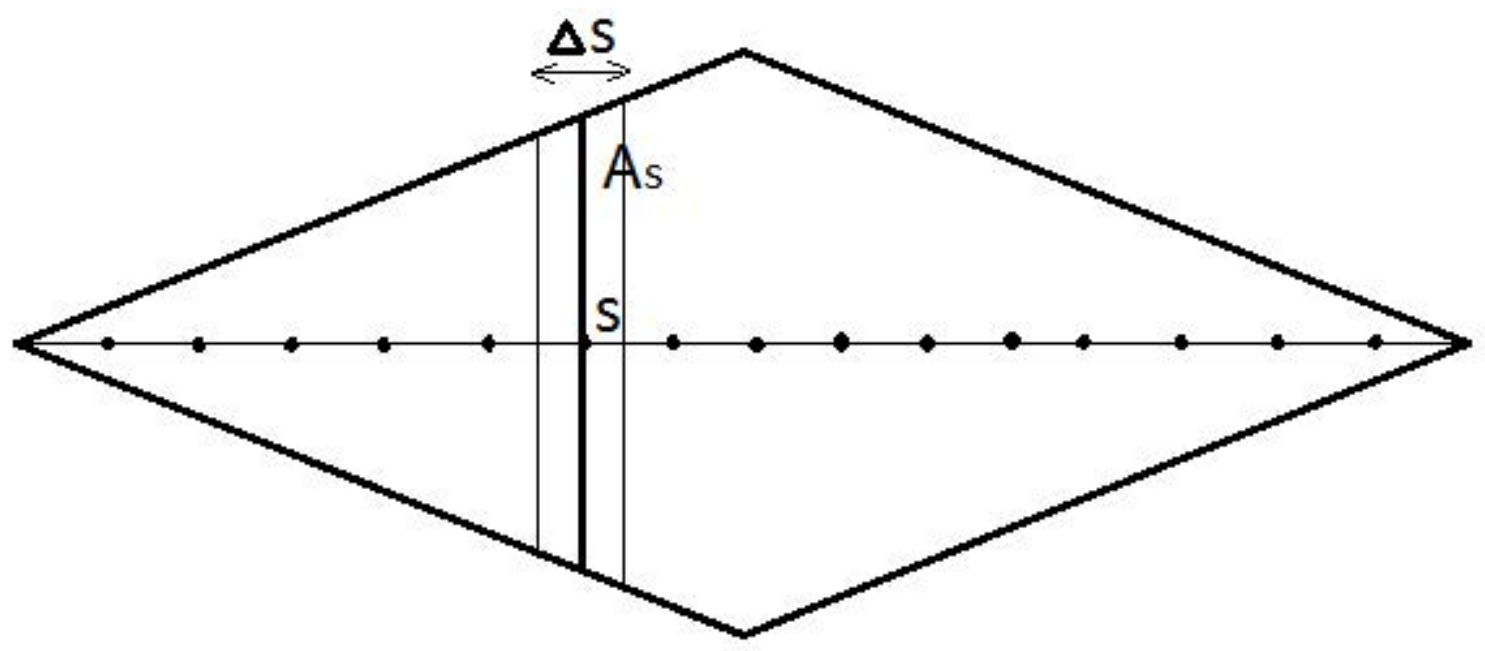

Figure 6.2: Representation of the area open to flow and $\Delta s$ along the central streamline path.

time of flight along the central streamline within the bond can be calculated by:

$$
\Delta t_{e, i}=\frac{\phi \Delta s}{u_{t}(s)}
$$

where $u_{t}(s)$ is velocity and $\phi$ is porosity. Batycky (1997) says that: "the time of flight (TOF) is the time required to reach a distance $s$ along a streamline based on the velocity field along the streamline" and he defined this as:

$$
\tau(s)=\int_{0}^{s} \frac{\phi(\zeta)}{u_{t}(\zeta)} \mathrm{d} \zeta
$$

In this work, the time of flight is the summation of incremental times of flight using Eq. 6.17, which is:

$$
\tau=\sum_{i=1}^{n} \Delta t_{e, i}
$$

The time of flight of the central streamline is a function of the volume of the associated bond. Integration over a distance $s$ along the central streamline gives the volume of the bond, $V_{s t}(s)$ :

$$
V_{s t}(s)=\int_{0}^{s} \phi(\zeta) A(\zeta) \mathrm{d} \zeta
$$


where $\zeta$ is a coordinate defined along the central streamline, and $A(\zeta)$ is the cross-sectional area of the tube. Using the definition of time of flight and knowledge of constant flux along the tube, Eq. 6.20 may be written as:

$$
V_{s t}(s)=\int_{0}^{s} \phi(\zeta) A(\zeta) \mathrm{d} \zeta=\int_{0}^{s} q_{s t} \frac{\phi(\zeta)}{u(\zeta)}=q_{s t} \tau(s)
$$

Now a value of flux, $q_{s t}$, can be assigned to the central streamline and this streamline can be used instead of the bond. Upcoming sections show the advantages of working with this streamline as opposed to the bonds and the simplicity of using time of flight instead of the volume of the bond.

\subsection{Mapping Saturation along Streamline and Fractional Flow}

In the two-phase network model, fluid flows in a single bond from the injector to the producer. The relation between bond volume and the central streamline time of flight was discussed in the previous section. For homogeneous cases with constant flux between injector-producer pairs, a larger bond volume results in a longer time of flight, and a smaller volume results in shorter time of flight. Velocity of the front between the displacing and displaced fluid and breakthrough time of the displacing fluid differs in different cases. Figure 6.3 illustrates the effect of a narrower crosssection and shorter time of flight on front position. With the same flux, the front has advanced more in the narrower case.

To find the saturation profile between an injector-producer pair, a saturation solution is mapped along a streamline. To do so, Batycky (1997) transformed the governing $3 D$ saturation equation into a pseudo- $1 D$ equation:

$$
\frac{\partial S_{i}}{\partial t}+\frac{\partial f_{j}}{\partial \tau}=0
$$

Eq. 6.22 may be solved either analytically or numerically. The numerical solution to Eq. 6.22 is generally the preferred solution due to its ability to adapt to existing saturation conditions when streamlines are updated during the simulation. 

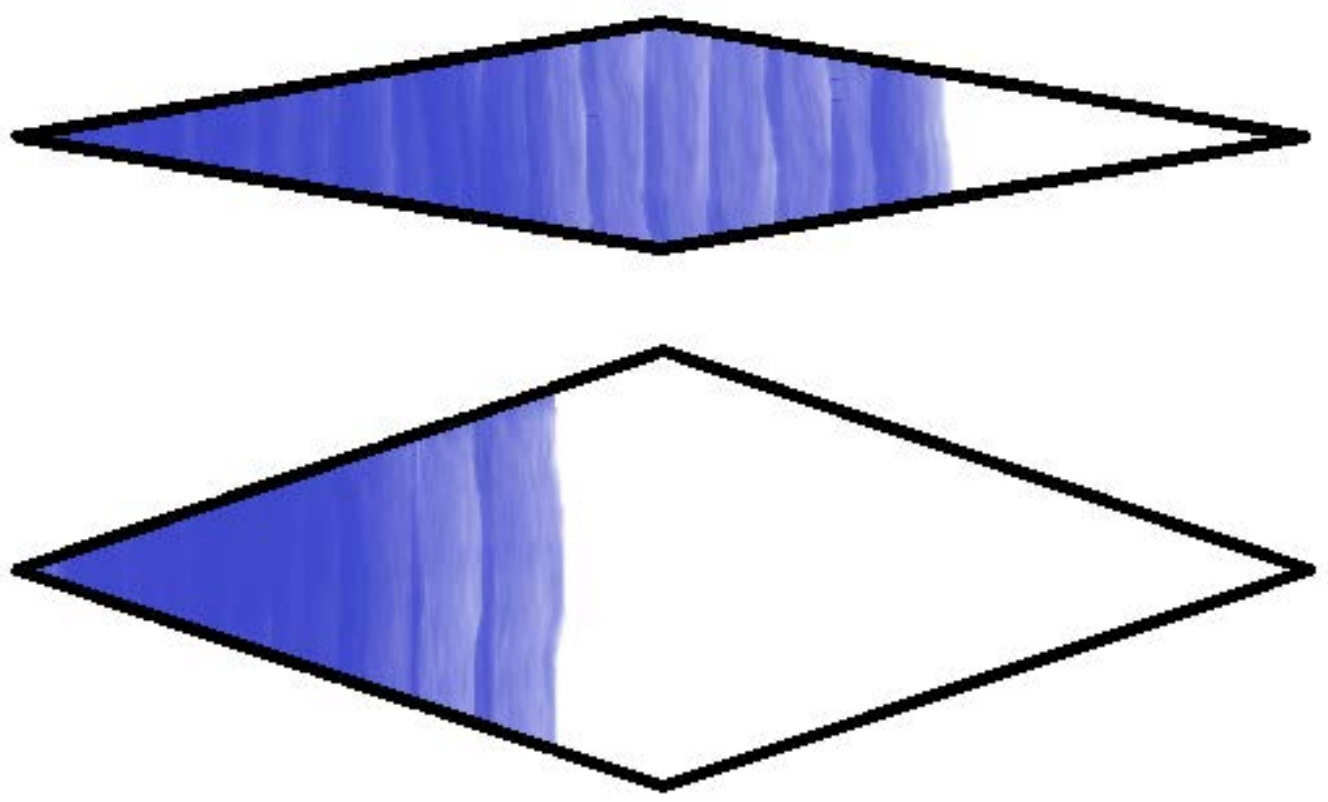

Figure 6.3: Flood front has advanced to different distance from injector in different cross-section tubes.

The procedure to map a $1 D$ numerical solution along a streamline is explained in detail by Batycky (1997). Steps are briefly discussed here with minor modifications.

1. Pick up saturation values from the underlying grid system and assign saturation values to the streamline path

2. Assign a time-of-flight grid onto the streamline path and obtain saturation at each time-offlight grid block (Figure 6.4b)

3. Transform the irregular time-of-flight grid in step 2 to a regular-time grid (Figure 6.4c)

4. Solve $1 D$ numerical equation on the regular-time grid and move saturations forward by $\Delta t$

5. Transform the regular-time grid back to the irregular time-of-flight grid (Figure 6.4e)

6. Assign new saturation value to the streamline path and to the underlying grid

In conventional streamline simulation, a streamline moves through different grid blocks to reach the producer. The grid blocks have different saturation values and times of flight. For example, the 


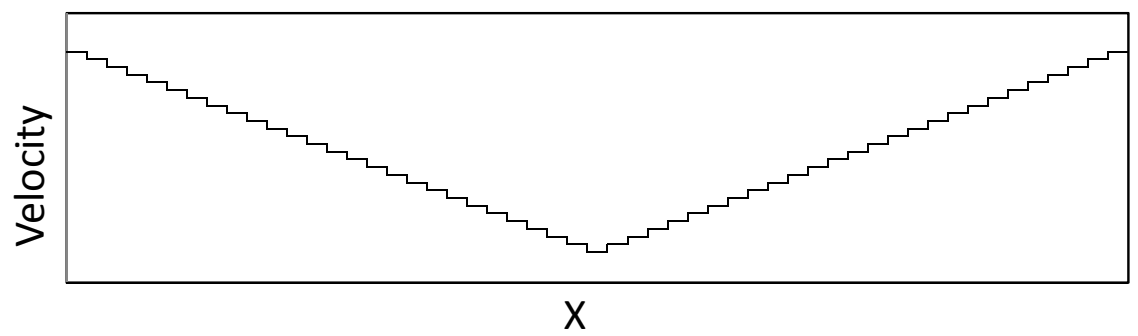

(a) Velocity profile along a tube.

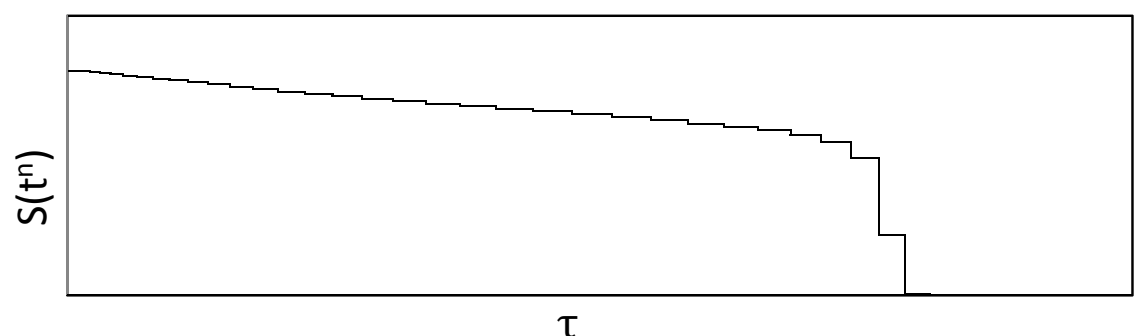

(b) Saturation on irregular $\tau$ grids at $t^{n}$.

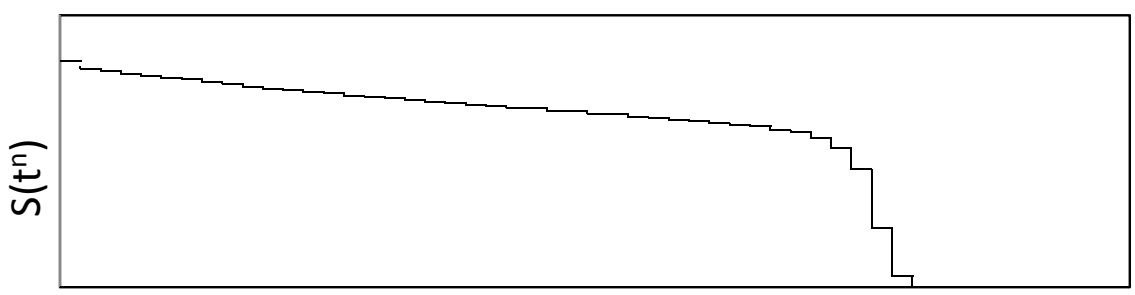

$\tau$

(c) Saturation on regular $\tau$ grids at $t^{n}$.

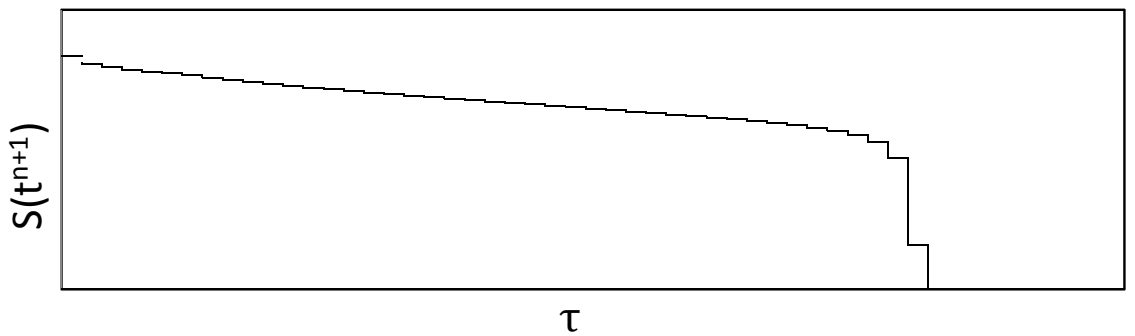

(d) Saturation on regular $\tau$ grids at $t^{n+1}$.

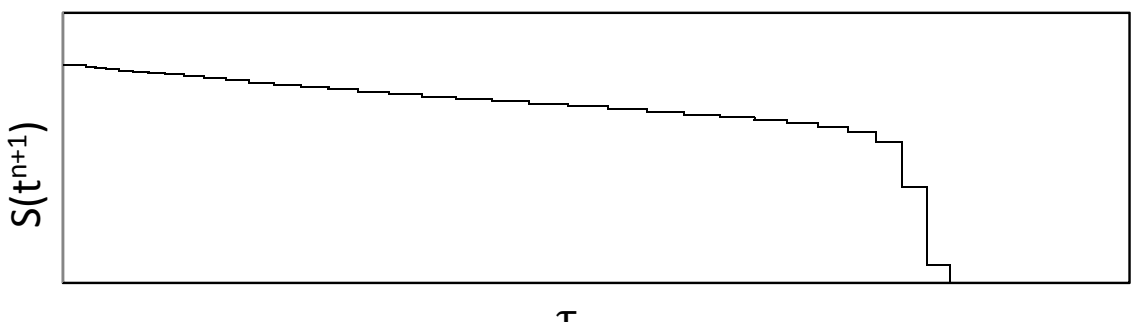

(e) Saturation on irregular $\tau$ grids at $t^{n+1}$.

Figure 6.4: Moving the saturation forward along streamline by $\Delta t$ step. 
time of flight is smaller in the grid blocks near injectors and producers where the velocity is higher. Therefore there will be smaller $\tau$ grid cells in higher flow velocity regions and larger $\tau$ grid cells in lower flow velocity regions.

In the network model, the maximum bond cross-section area open to flow (corresponding to the widest point in the diamond shape) is assumed, and streamlines within this area are launched. Saturation and time of flight are assigned to equally distanced grid points along the center streamline path between injectors and producers (Figure 6.2). Although the points are equally distanced, there is a lower resolution in $\tau$ space near injectors and producers. Therefore, $S$ versus $\tau$ information is defined on irregular grids. Using regular $\tau$ grids makes it much easier to use Eq. 6.22. The same number of grid points is used for both regular and irregular grids. In order to make sure that mass is conserved in the transformation process, Eq. 6.23 needs to hold between regular and irregular grids (Batycky 1997):

$$
\left.\int_{0}^{s} S_{j} \mathrm{~d} \tau\right|_{\text {regular }}=\left.\int_{0}^{s} S_{j} \mathrm{~d} \tau\right|_{\text {irregular }}
$$

The time derivative of the hyperbolic PDE in Eq. 6.22 is approximated by:

$$
\frac{\partial S}{\partial t} \sim \frac{S^{n+1}-S^{n}}{\Delta t}
$$

In $\tau$ space, the derivative is approximated by a forward difference method as shown below:

$$
\frac{\partial f}{\partial \tau} \sim \frac{f_{i}^{n+1}-f_{i}^{n}}{\Delta \tau}
$$

Discretization of Eq. 6.24 using Eq. 6.25 and Eq. 6.22 yields:

$$
S_{i}^{n+1}=S_{i}^{n}-\frac{\Delta t_{s l}}{\Delta \tau}\left(f_{i}^{n+1}-f_{i}^{n}\right)
$$

Where $\Delta t_{s l}$ is the current time step size along a stramline and $\Delta \tau$ is the time-of-flight distance between nodes for a given streamline. 
It is worth mentioning that the streamline time step, $\Delta t_{s l}$, is different from the network model time step, $\Delta t$. In the network model, the time step is the time interval in which production and injection data are recorded. The streamline time step should be less than or equal to the network model time step, and equation Eq. 6.26 is solved $n$ times where $n$ is:

$$
n=\frac{\Delta t}{\Delta t_{s l}}
$$

In addition, stability analysis of the finite difference equation shows the scheme is unstable if the diffusion coefficient is negative (Mattax and Dalton 1990). The diffusion coefficient is a function of $\Delta \tau$ and $\Delta t_{s l}$ an written as:

$$
D=\frac{f^{\prime}}{2}\left(\Delta \tau-f^{\prime} \Delta t\right)
$$

where $f$ represent the fastest saturation velocity, which is the saturation of front. Therefore, the maximum time step for Eq. 6.26 is defined as:

$$
\frac{\Delta t_{s l}}{\Delta \tau} \leq \frac{1}{f^{\prime}}
$$

This condition does not let the fastest saturation velocity travel more than one $\tau$ node per time step.

\subsection{Two-Phase Flow Parameters}

By mapping saturation on streamlines one can determine the fractional flow values along streamline paths that connect an injector to a producer. The fractional flow values are directly used in the well model and determine the relative permeability between node volumes. Figure 6.5 shows the desirable fractional flow values and their importance. In this figure, injector $A$, producer $B$, and the volume associated with one is illustrated. The diamond represents the bond associated with the center streamline that connects injector $A$ to producer $B$. The fractional flow at the producer is the fractional flow value of the last point on the streamline path (point B). In addition, it is assumed that the fractional flow at the interface between the two node volumes is equal to the fractional flow 


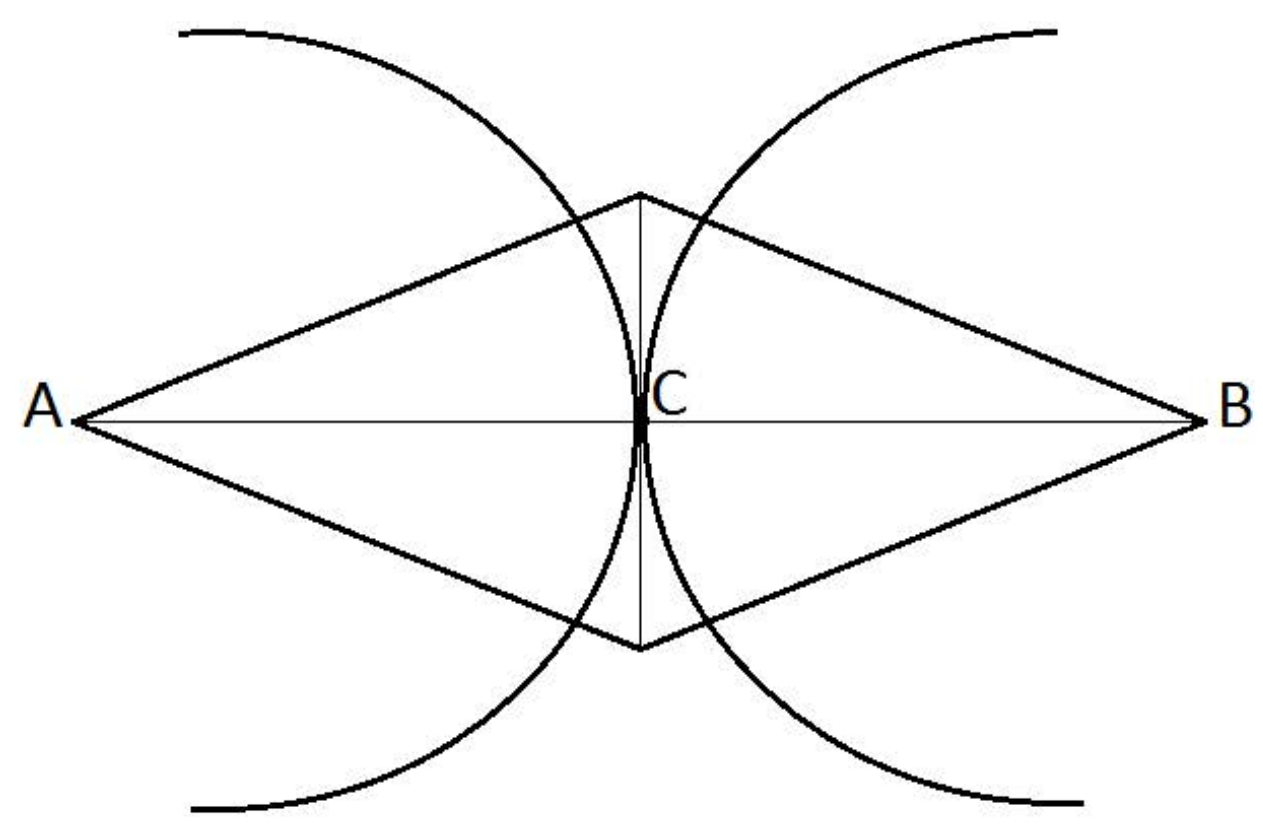

Figure 6.5: Node volume associated with injector $A$ and producer $B$ and the bond between them. Point $C$ is the interface between two volumes.

of the mid-point of the center streamline (point C). Knowing the fractional flow at the interface, one can obtain the fractional water and oil flow between nodes from:

$$
\begin{gathered}
q f_{p}=\operatorname{TRANS}_{w}\left(P_{A}-P_{B}\right) \\
q\left(1-f_{p}\right)=\operatorname{TRANS}_{o}\left(P_{A}-P_{B}\right)
\end{gathered}
$$

$T R A N S_{w}$ and $T R A N S_{o}$ are functions of phase conductivities. By knowing the fractional flow of water at each step, they can be easily calculated.

\subsection{Limitations of Single Streamline Method}

The problem that is solved here is similar to the Buckley-Leverett fractional flow theory. In this system there is a varying cross-sectional area instead of a constant one. Thus a numerical method is required to solve the equation. The numerical solution, like the analytical one, produces a sharp front where low saturation values are swept by the front (Welge 1952). This produces a sudden sharp 
rise in the water fractional flow on breakthrough. In multi-dimensional systems, breakthrough corresponds to the shock front reaching the producer through the fastest streamline. In these cases, instead of a sharp rise in fractional flow, a more gradual rise in fractional flow is usually observed (Malik 1988).

Figure 6.6 compares field data fractional flow and model fractional flow on a similar case. Unlike the gradual increase in water cut in field data, a sharp increase in water cut in fractional flow is observed. This comparison implies that matching water fractional flow with frontal advance theory would be very difficult. It will be even harder if the media is heterogeneous, like a layered reservoir. In order to resolve this issue, another parameter is added to reduce the effect of the shock in homogeneous media and enable the method to handle heterogeneous cases.

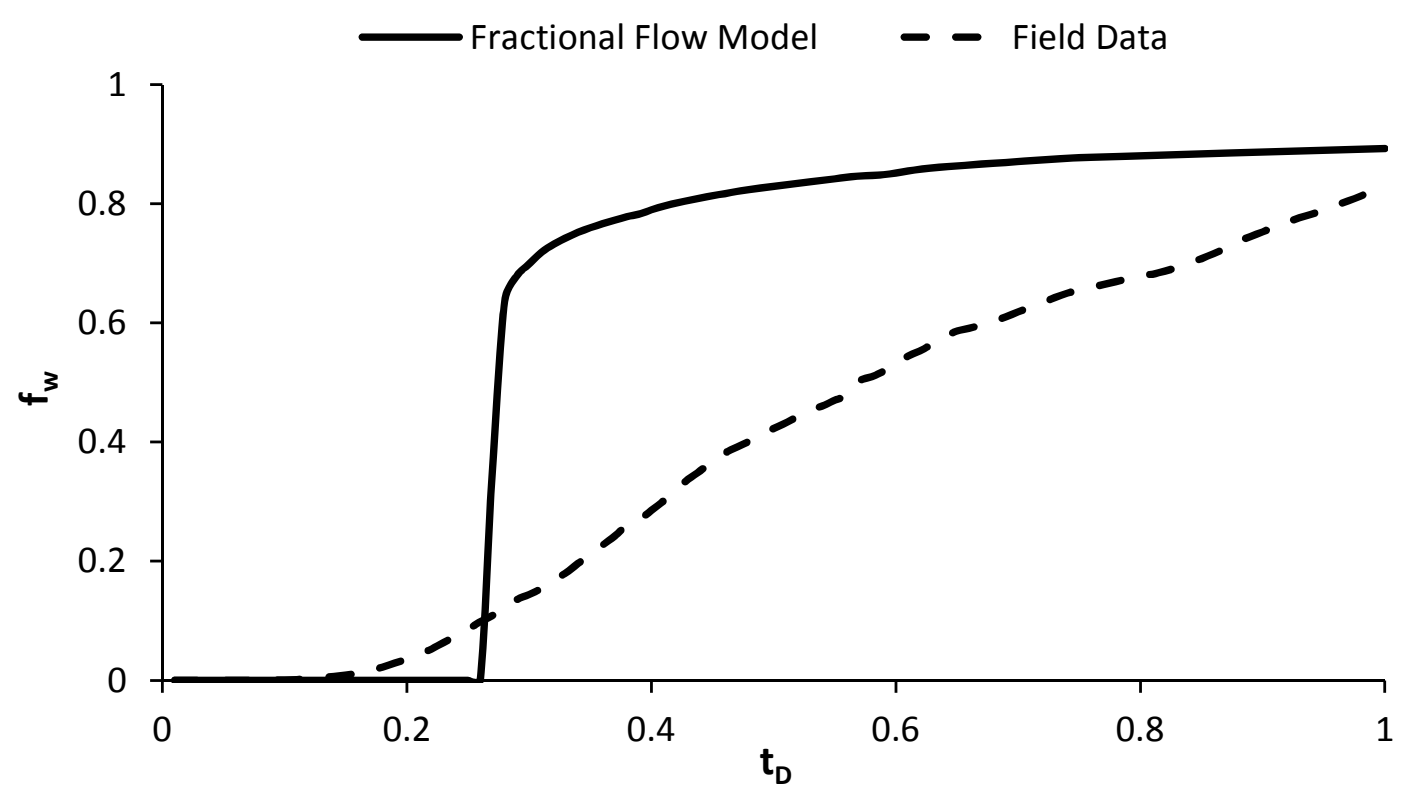

Figure 6.6: Difference between observed fractional flow and model fractional flow.

\subsection{Dykstra-Parsons Method}

The Dykstra-Parsons method was described for waterflooding in a stratified porous media. Dykstra and Parsons (1950) assumed that a reservoir is composed of a number of non-communicative homogenous layers and that each layer has different properties from other layers. Water displaces 
oil in a piston-like manner in each layer, and both fluids are incompressible. In addition, they characterized permeability by a log-normal distribution by assuming permeability to be a random function with a known mean, variance, and spatial correlation. Based on these assumptions, they introduced a method for describing the distribution of absolute permeability of reservoir rocks determined from core data. The Dykstra-Parsons, $V_{D P}$, is equal to

$$
V_{D P}=\frac{k_{50}-k_{84.1}}{k_{50}}
$$

where $k_{50}$ is the median permeability, and $k_{84.1}$ is the permeability value one standard deviation lower than the median. Figure 6.7 is a plot of the logarithm of permeability versus the percentage of permeability larger than that particular permeability value. Another application of the DykstraParsons coefficient is to obtain permeability of layers when the Dykstra-Parsons coefficient is known (Zemel 1995). Pande et al. (1987) used the Dykstra-Parsons model to determine a permeability distribution from displacement data.

This method is generally applied to the property of permeability but can be extended to treat other rock properties (Ahmed 2010). Here this concept is applied to the time of flight. It is assumed

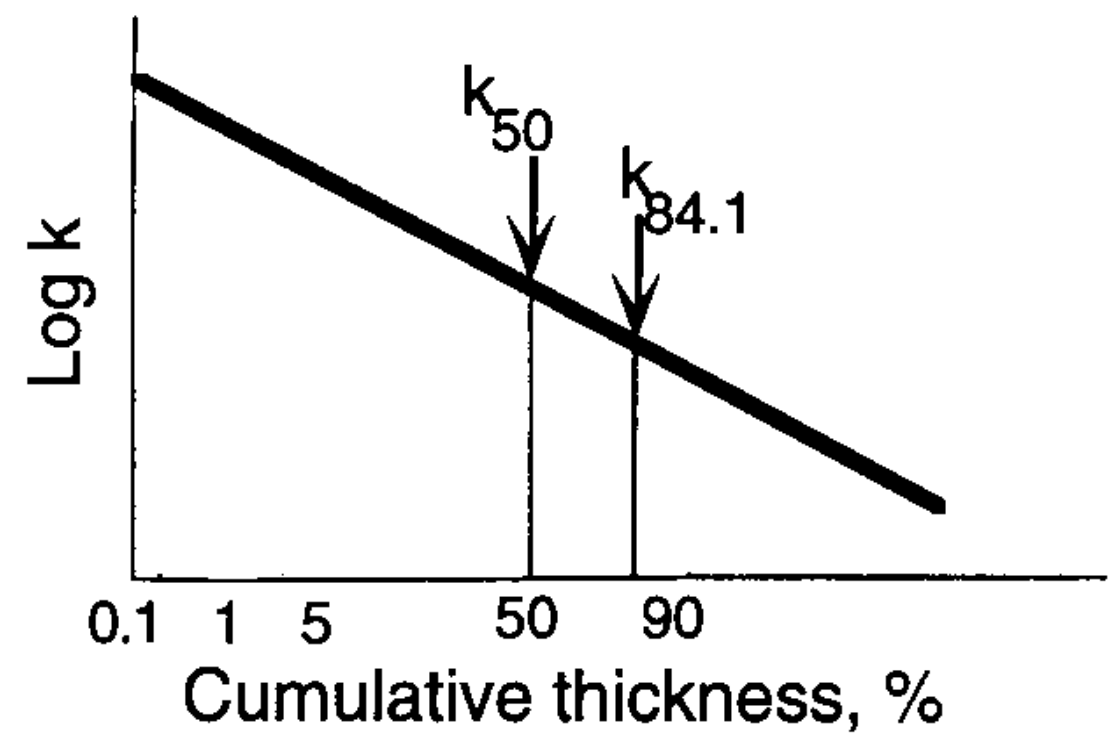

Figure 6.7: Dykstra-Parsons cumulative probability plot (Zemel 1995). 
that the saturation is moving along streamlines inside the network model bond with different times of flight. This is a valid assumption for both homogeneous and heterogeneous reservoirs. In addition, it is assumed that these times of flight can be characterized by a log-normal distribution, and therefore the probability that the time of flight is equal to that of $\ln (\tau)$ is given by the probability density function for a log-normal distribution:

$$
p(z=\ln (\tau))=\frac{1}{\sqrt{2 \pi \sigma}} \exp \left\{-\frac{\left(\ln (\tau)-\ln \left(\tau_{50}\right)\right)^{2}}{2 \sigma^{2}}\right\}
$$

Where

$$
\sigma=\ln \left(\tau_{50}\right)-\ln \left(\tau_{84.1}\right)=-\ln \left\{\frac{\tau_{84.1}}{\left(\tau_{50}\right)}\right\}=-\ln \left(1-V_{\tau}\right)
$$

$\tau_{50}$ is the median time of flight, which is the time of flight of the central streamline that connects an injector to a producer and $V_{\tau}$ is a time-of-flight Dykstra-Parsons coefficient that characterizes flow in the bond. The probability that the time of flight is larger than the value of $\ln (\tau)$ is obtained from the cumulative distribution function:

$$
p(z>\ln (\tau))=\frac{1}{2}\left\{1-\operatorname{erf}\left(-\frac{\ln \frac{\tau}{\ln \tau_{50}}}{\ln \left(1-V_{\tau}\right)}\right)\right\}
$$

If flow from the injector to the producer is represented by $n$ streamlines inside each tube, and the probability that the time of flight of streamline $j$ is greater than $\tau$ is:

$$
p=\frac{j}{n}
$$

Therefore from Eq. 6.35 the time of flight of streamline $j$ would be:

$$
\frac{\tau_{j}}{\tau_{50}}=\left(1-V_{\tau}\right)^{-\sqrt{2} e r f^{-1}\left(1-2 p_{j}\right)}
$$

Where $\operatorname{erf}^{-1}$ is the inverse of the error function. Knowing $\tau_{50}$ and $p$ terms, the time of $n$ streamline are calculated from Eq. 6.37. 


\subsection{Average Fractional Flow at Producers and Interfaces}

In each tube of the network model there are multiple fractional flow values that pass across interfaces and arrive at producers. Flux carried by each streamline within each tube is used to calculate the average fractional flow values (Batycky 1997). Fractional flow at the interface and at the producer, $f_{\text {inter }}$ and $f_{p}$, are given by:

$$
\begin{gathered}
f_{p}=\frac{\sum_{i=1}^{n} q_{i}^{s l} f_{p_{i}}^{s l}}{\sum_{i=1}^{n} q_{i}^{s l}} \\
f_{\text {inter }}=\frac{\sum_{i=1}^{n} q_{i}^{s l} f_{\text {inter }_{i}}^{s l}}{\sum_{i=1}^{n} q_{i}^{s l}}
\end{gathered}
$$

Where $f_{\text {inter }}$ and $f_{p_{i}}$ are the fractional flow of the $i^{\text {th }}$ streamline at the interface and the producer, respectively. $q_{i}^{s l}$ is the flux carried by each streamline and is given by:

$$
q^{s l}=\frac{q_{I J}}{n}
$$

$q_{I J}$ represents flux between injector $I$ and producer $J$, and $n$ is the number of streamlines between them. The number of streamlines between each injector-producer pair is assumed to be constant in the network model and is not a function of the flux between them. In addition, the number of streamlines that launch from injectors is equal and not a function of injection rates. The number of streamlines between injector-producer pairs will be discussed in the upcoming sections.

\subsection{Verification}

To verify, the model's saturation distribution is compared with that of a Buckley-Leverett problem. The test case is a linear bed with a throughput of $95 \mathrm{bbl} /$ day at the injector side and production at the opposite side, which is $800 \mathrm{ft}$ apart. The cross-sectional area is the product of width $25 \mathrm{ft}$, and the formation thickness, $10 f t$.

The dimensionless form of the Buckley-Leverett equation may be written as: 


$$
\frac{x_{D}}{t_{D}}=\left(\frac{\partial f_{w}}{\partial S_{w}}\right)_{S_{w}}
$$

Where $x_{D}$ and $t_{D}$, dimensionless length and dimensionless time, are defined as (Thiele et al. 1996):

$$
x_{D}=\int_{0}^{s} \frac{\phi(\zeta) A(\zeta) d \zeta}{\bar{V}_{p}}
$$

and

$$
t_{D}=\int_{0}^{t} \frac{q d t}{\bar{V}_{p}}
$$

By starting from equation Eq. 6.41 and using Eq. 6.42 and Eq. 6.42 one obtains (Thiele et al. 1996),

$$
\frac{x_{D}}{t_{D}}=\frac{\int_{0}^{s} \phi(\zeta) A(\zeta) d \zeta}{q t}=\frac{1}{t} \int_{0}^{s} \frac{\phi(\zeta) A(\zeta) d \zeta}{v(\zeta) \phi(\zeta) A(\zeta)}=\frac{1}{t} \int_{0}^{s} \frac{d \zeta}{v(\zeta)}=\frac{\tau(s)}{t}
$$

Therefore the Buckley-Leverett equation may be written as:

$$
\frac{\tau}{t}=\left(\frac{\partial f_{w}}{\partial S_{w}}\right)_{S_{w}}
$$

Each point on the distance between an injector-producer pair corresponds to a $\frac{\tau}{t}$. The equation below is used to assign a saturation value to each point.

$$
S_{w}=\left\{\begin{aligned}
S_{w i r} & \text { if } \frac{\tau}{t}>\left(\frac{\partial f_{w}}{\partial S_{w}}\right)_{S_{w_{f r o n t}}} \\
f_{w}^{\prime-1}\left(\frac{\tau}{t}\right) & \text { if } \frac{\tau}{t}<\left(\frac{\partial f_{w}}{\partial S_{w}}\right)_{S_{w_{f r o n t}}}
\end{aligned}\right.
$$

where ${f^{\prime}}_{w}^{-1}$ is the inverse of derivative fractional flow.

Saturation is mapped on a single center streamline connecting the injector to the producer, and the result is shown in Figure 6.8. Figure 6.8 shows the agreement in front position for both BuckleyLeverett method and saturation mapping. 


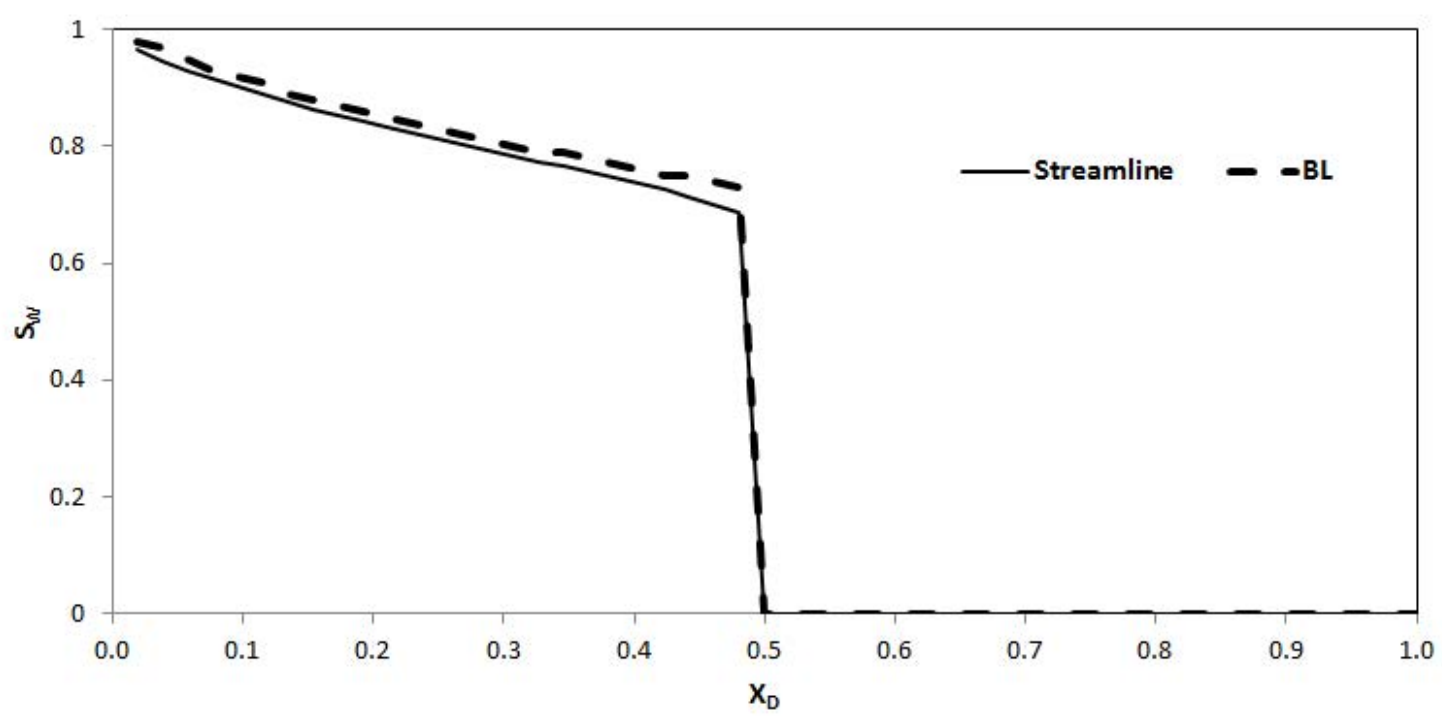

Figure 6.8: Verification of the model with Buckley-Leverett.

\subsection{Application}

In Chapter 3, the total fluid production was matched with the observed synthetic data to obtain $k A_{c s}$, the permeability and area product. In this section, individual phase production is matched with observed synthetic data to determine the average time of flight between well-pairs and heterogeneity of the reservoir. More specifically, the cross-sectional area open to flow, $A_{c s}$, and the time-of-flight Dykstra-Parsons coefficient are matched to fulfill this goal. The first step is a preliminarily step to the second step. Therefore, for each case in this study, a single-phase network model is run to find $k A_{c s}$ values. $k A_{c s}$ values are substituted into the two-phase network model, where the unknowns then are phase conductivity values at the node interfaces and at the producers. Values of the cross-sectional area open to flow and the time-of-flight Dykstra-Parsons coefficient give phase conductivity values that match individual phase production.

To start, the method is applied on two linear bed models with one injector and one producer. Rate fluctuates at the injector, and bottomhole pressure is kept constant at the producer. The crosssectional area of the model is $880 \mathrm{ft}$ by $60 \mathrm{ft}$, and it is $1680 \mathrm{ft}$ long. The permeability is a constant $40 \mathrm{md}$ in the first model. The permeability in the second case has $40 \mathrm{md}$ everywhere except the center set of grid cells, which have $400 \mathrm{md}$. The wells are placed in a $400 \mathrm{md}$ set of blocks (Figure 
6.9). Table 6.1 shows obtained values of $k A_{c s}, A_{c s}$, and $V_{\tau}$ and Figures 6.10 and 6.11 compare obtained fractional flow with observed data for each case.

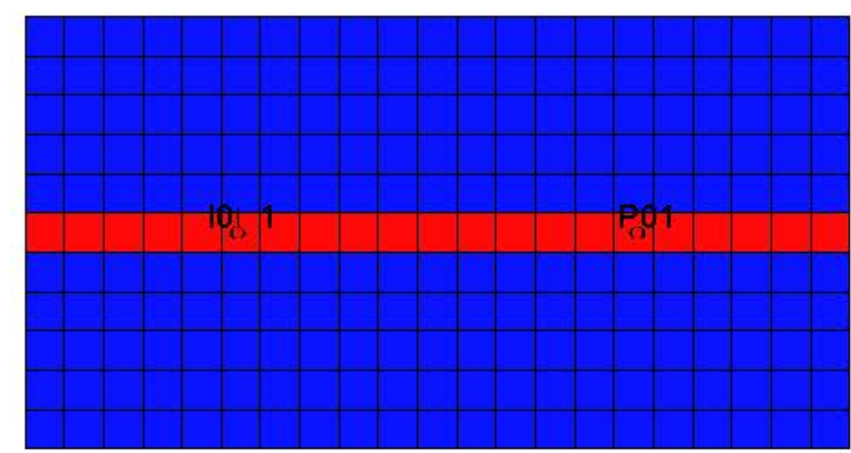

PermX(MDARCY)

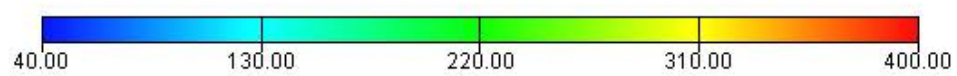

Figure 6.9: $1 D$ Model with highly permeable channel.

Table 6.1: Comparisons of two homogeneous and hetrogeneous linear cases

\begin{tabular}{lccc}
\hline & $k A_{c s}$ & $A_{c s}$ & $V_{\tau}$ \\
\hline Homogeneous Model & 20 & 1455 & 0.48 \\
\hline Heterogeneous Model & 42 & 410 & 0.97 \\
\hline
\end{tabular}

Values for $k A_{s c}$ are obtained from the single phase-network model and show that the heterogeneous model has a $k A_{s c}$ values twice that of the homogeneous one. This result was expected, since the dimensions of the model were kept constant, while average permeability was increased. The conductivity comparison does not tell anything about the nature of the heterogeneity. But values of the $A_{c s}$ and $V_{\tau}$ demonstrate the difference between the two models very well. $A_{c s}$ is much smaller comparatively, reflecting the fact that fluid flows through the channel, and $V_{\tau}$ is double the value of the homogeneous case, showing the heterogeneity of the model. One may ask why $V_{\tau}$ is not zero in the homogeneous case. The answer is that a 0 value corresponds to piston-like displacement, and flow, even in a homogeneous model, is not piston-like. In addition, this study is more a relative comparison of similar cases, and a single value by itself may not say much. 


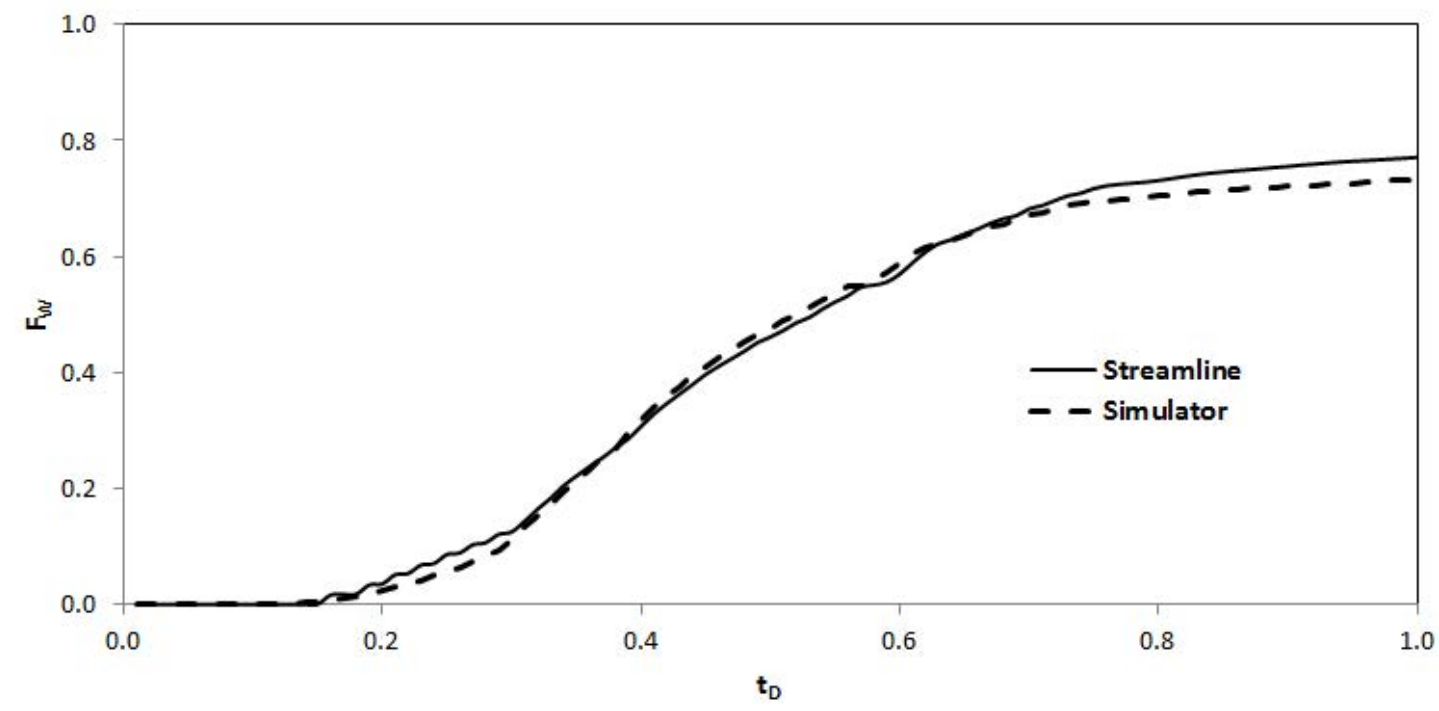

Figure 6.10: Fractional flow at producer versus dimensionless time for homogeneous case.

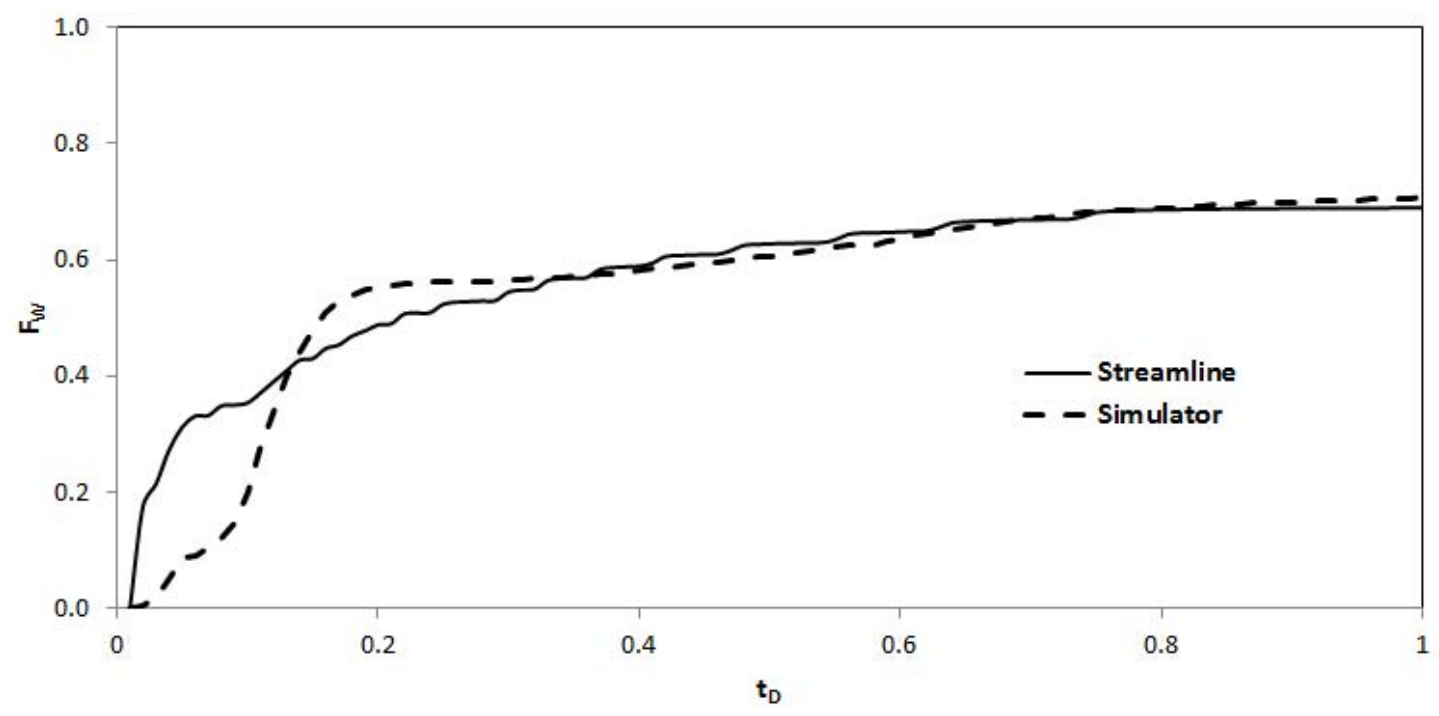

Figure 6.11: Fractional flow at producer versus dimensionless time for the case with channel.

To show the effect of heterogeneity on the $V_{\tau}$ and $A_{c s}$ more clearly, heterogeneity is represented by variance of layer permeability values. In the next comparison, the same $1 D$ example was extended to include permeability variations in the vertical direction. Nine equal thickness layers, with thicknesses adding up to $60 \mathrm{ft}$, have different permeability values that represent vertical heterogeneity. Variances of layer permeability values show the degree of heterogeneity. Figure 6.12 shows the structure of the model for the highest permeability variance. Both injector and producer 
are perforated through all layers. Unlike the assumptions of the Dykstra-Parsons coefficient, layers are communicating, and vertical permeability is $4 \mathrm{md}$.

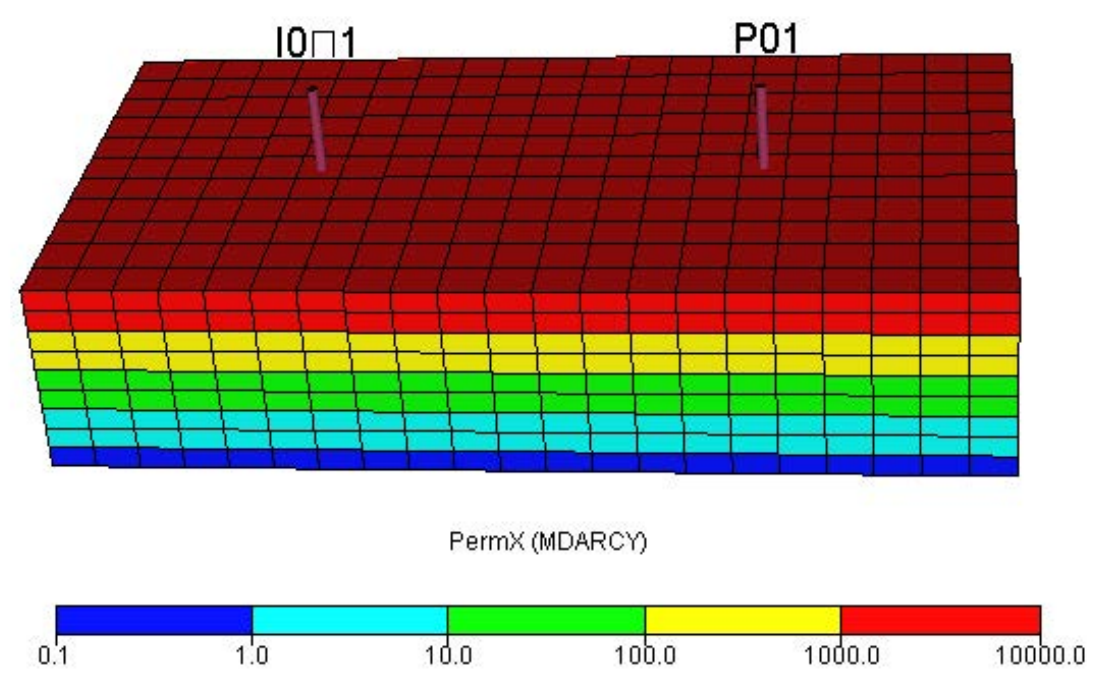

Figure 6.12: 9 layers case: Heterogeneity in vertical direction.

Table 6.3 shows the obtained values of $A_{c s}$ and $V_{\tau}$ for the different values of permeability variance. The result shows that when permeability variance increases, $A_{c s}$ decreases, and $V_{\tau}$ increases. Higher $V_{\tau}$ for higher heterogeneity was expected. Reduction of $A_{c s}$ reflects the fact that smaller portions of the model are flooded. In other words, a reduction of $A_{c s}$ means low sweep efficiency. In Table 6.3, sweep efficiency is represented by the field average water saturation. This is a valid assumption, since lower efficiency floods leave more oil behind, and average water saturation is lower. The field average water saturation decreases with increased reservoir heterogeneity and reduction of $A_{c s}$. Therefore $A_{c s}$ may be a good representative of the sweep efficiency between injector-producer well-pairs. Figure 6.13 and Figure 6.14 illustrate the effect of heterogeneity on $V_{\tau}$ and $A_{c s}$ respectively.

The second pair of models are two-dimensional $1240 \mathrm{ft}$ by $1240 \mathrm{ft}$ models with one injector in the middle and 4 producers at the corners. The difference between them is the more permeable diagonal region that passes from the injector and producer 1 and producer 4 in the second model (Figure 6.15). The permeability in that region is double that in the rest of the model. Table 6.3 and 
Table 6.2: Obtained parameter values for heterogeneous $2 D$ model

\begin{tabular}{lcccc}
\hline Variance & $k A_{c s}$ & $A_{c s}$ & $V_{\tau}$ & $S_{w_{\text {ave }}}$ \\
\hline 5 & 20.4 & 1463 & 0.495 & 0.588 \\
\hline 22 & 20.6 & 1464 & 0.496 & 0.587 \\
\hline 154 & 22.2 & 1446 & 0.524 & 0.582 \\
\hline 795 & 29.1 & 1349 & 0.612 & 0.557 \\
\hline 7373 & 1179 & 978 & 0.708 & 0.474 \\
\hline 1924911 & 12811 & 444 & 0.798 & 0.336 \\
\hline
\end{tabular}

Table 6.4 show the obtained single-phase and multi-phase parameter values. Figure 6.16 shows that the model matches the fractional flow at the producers.

Table 6.3: Obtained parameter values for homogeneous model with an injector and 4 producers.

\begin{tabular}{cccc}
\hline & $k A_{c s}$ & $A_{c s}$ & $V_{\tau}$ \\
\hline P1 & 8.1 & 1029 & 0.378 \\
\hline P2 & 8.1 & 1026 & 0.371 \\
\hline P3 & 8.1 & 1027 & 0.375 \\
\hline P4 & 8.1 & 1028 & 0.376 \\
\hline
\end{tabular}

Table 6.4: Obtained parameter values for heterogeneous model with an injector and 4 producers.

\begin{tabular}{cccc}
\hline & $k A_{c s}$ & $A_{c s}$ & $V_{\tau}$ \\
\hline P1 & 26.1 & 1026 & 0.503 \\
\hline P2 & 12.4 & 917 & 0.331 \\
\hline P3 & 12.4 & 921 & 0.337 \\
\hline P4 & 26.1 & 1024 & 0.503 \\
\hline
\end{tabular}

The values in Table 6.3 reflect the homogeneity of the system and symmetry of the well placement. Values in Table 6.4 also show symmetry with respect to the diagonal axes. The values of $k A_{c s}$ in Table 6.4 are two times larger for producer 1 and producer 4 , showing the fact that those wells are connected to the injector with a more permeable region. $V_{\tau}$ values are also larger, showing that 


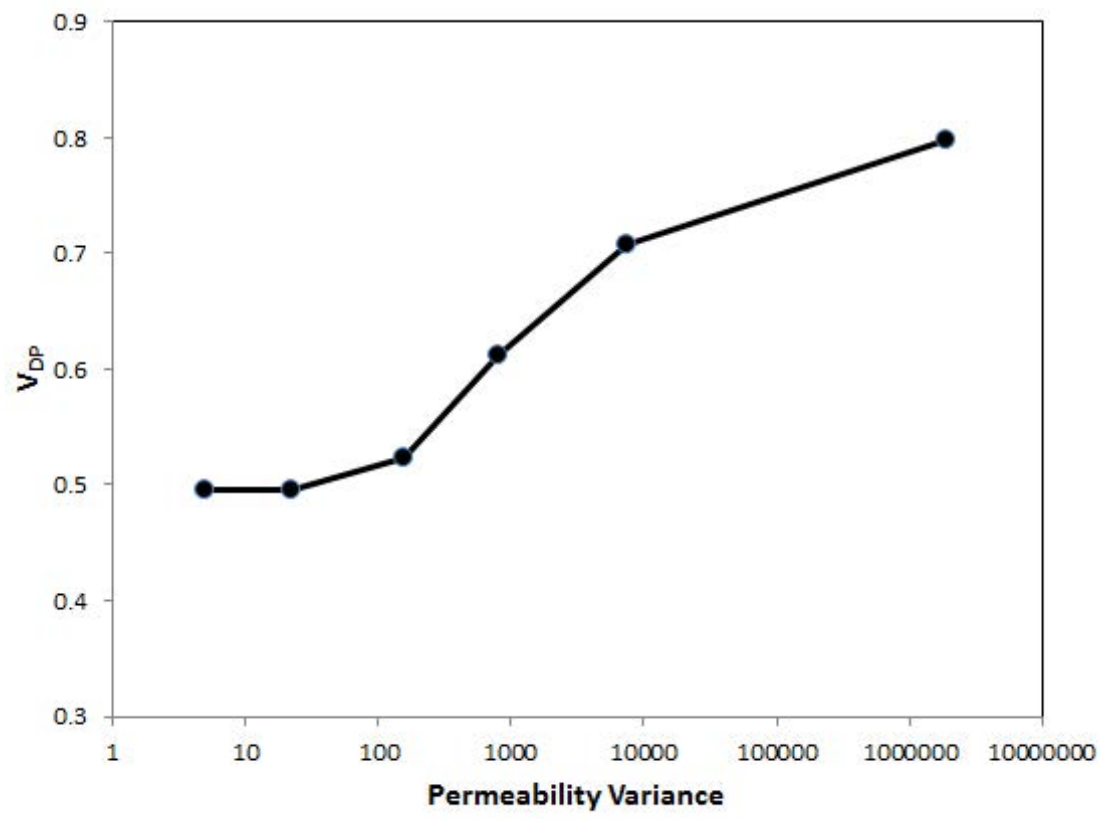

Figure 6.13: Increase in time-of-flight Dykstra-Parsons coefficient with increase in heterogeneity

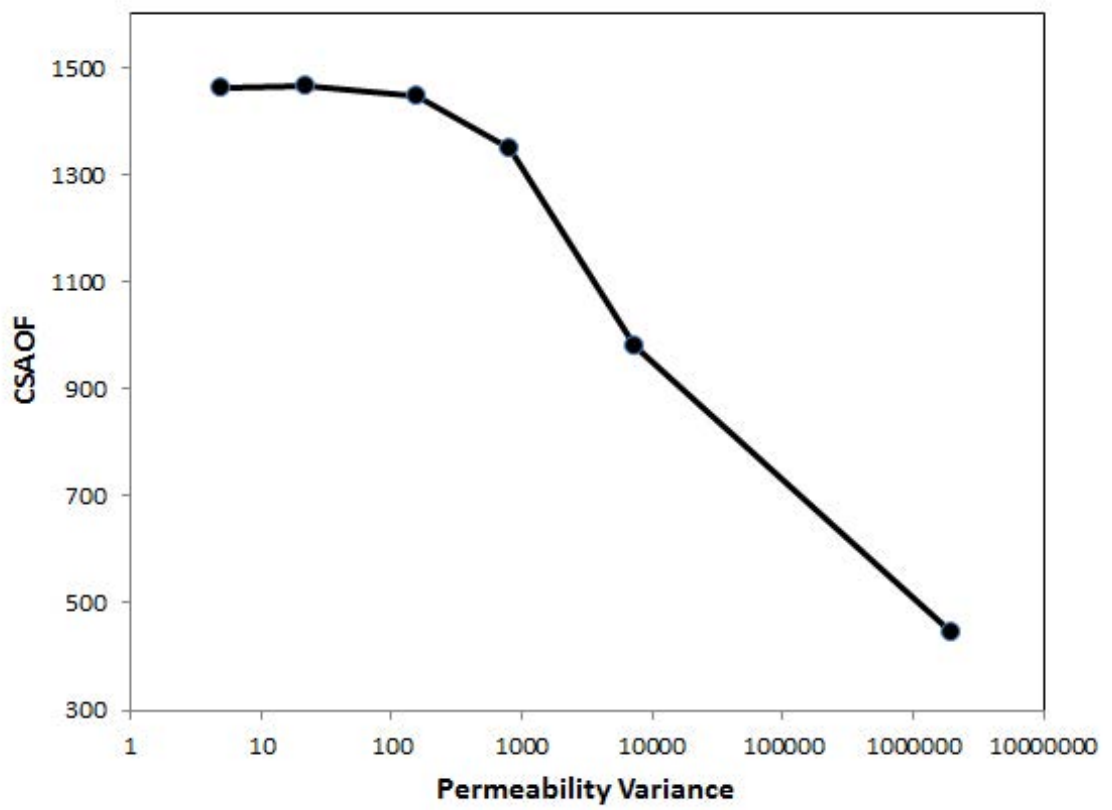

Figure 6.14: Reduction of cross section area open to flow with increase in heterogeneity

fluid flows through a more heterogeneous path to reach producer 1 and producer 4 . By comparing Table 6.3 and Table 6.4, one can notice that $K A_{c s}$ values for producer 2 and producer 3 are larger 


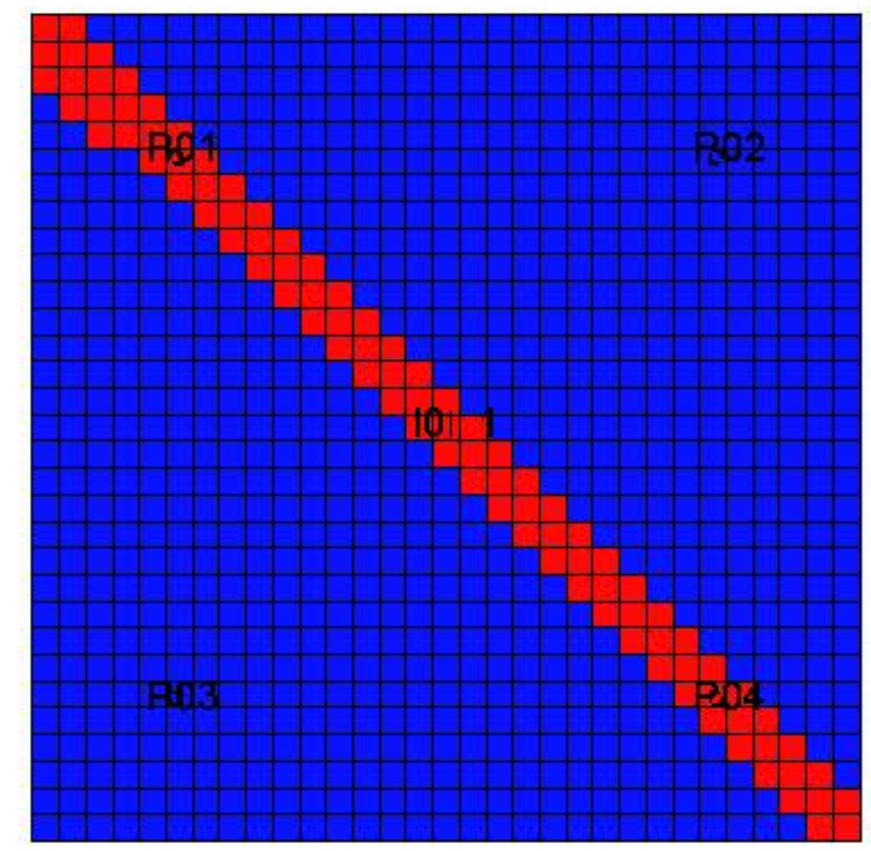

PermX (MDARCY)

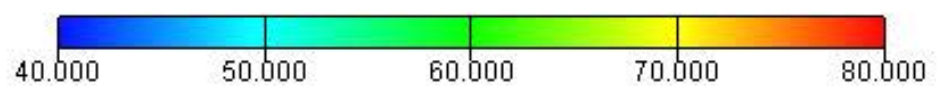

Figure 6.15: 2D Model with diagonal more permeabile region.

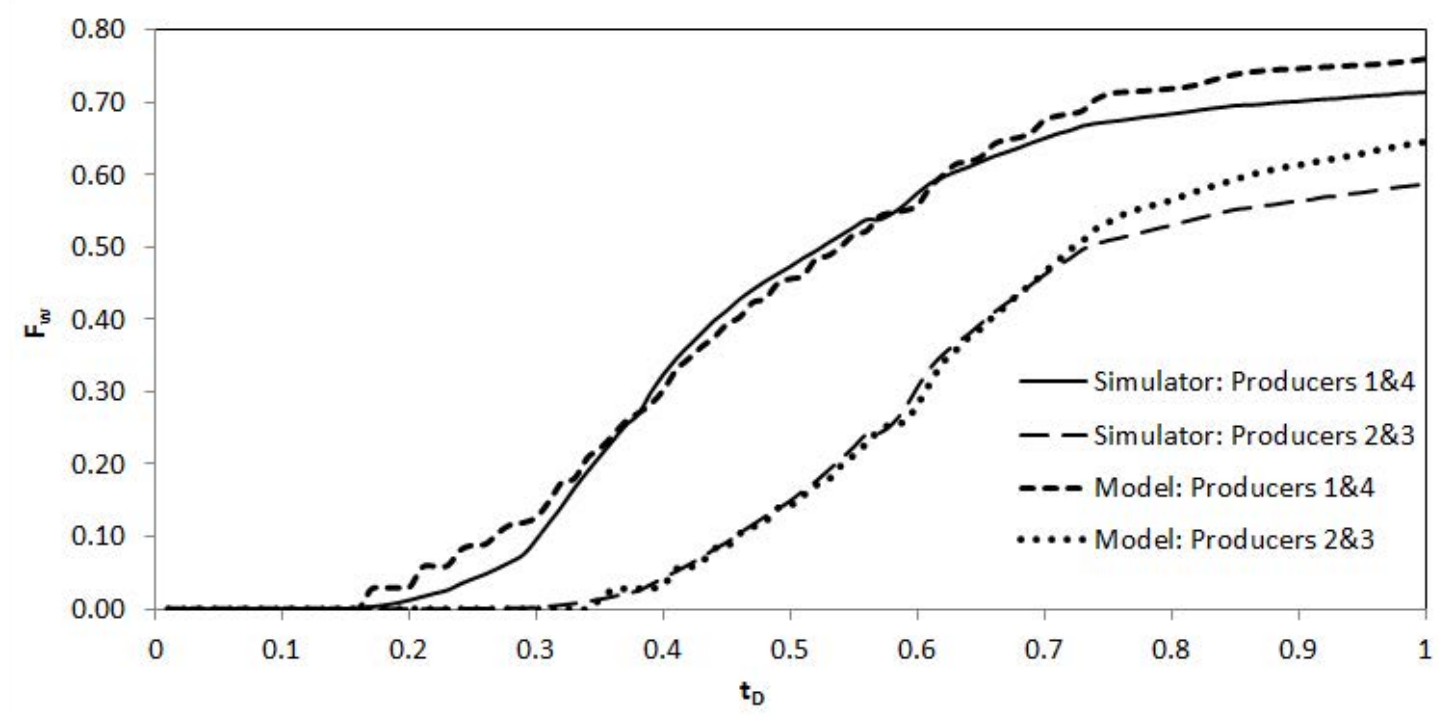

Figure 6.16: Fractional flow at producers versus dimensionless time for $2 D$ model with different permeability regions. 
in the heterogeneous case because the more permeable region probably has influenced those wells as well. But $V_{\tau}$ for producer 2 and 3 are the same in both models.

Fractional flow observations in all $1 D$ and $2 D$ examples that are studied in this chapter thus far comes from one injector. The problem becomes more complicated when there are multiple injectors in the system. In this case, observed fractional flow at the producer is the combination of multiple fractional flows from different injectors. This problem is set up by placing two injectors at both sides of the producer, as is shown in Figure 6.17. While injector 1 is connected to the producer through a highly permeable channel, the medium between injector 2 and the producer is homogeneous. Values of $k A_{c s}$ in table 6.5 show a higher permeability between injector 1 and the producer. Values of $V_{\tau}$ and $A_{c s}$ reflect more heterogeneity and less sweep between that injectorproducer pair.

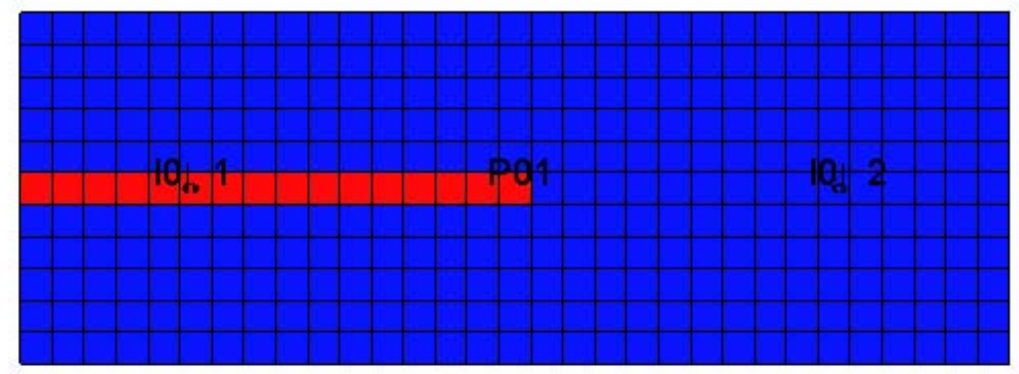

PermX(MDARCY)

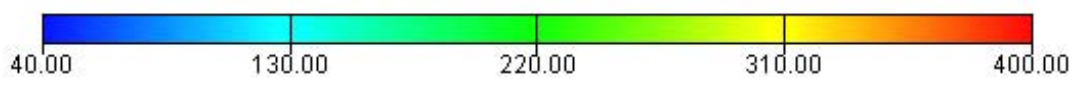

Figure 6.17: Model with two injector and a high permeability channel.

Table 6.5: Obtained parameter values for $2 D$ model with two injectors and a high permeable channel

\begin{tabular}{cccc}
\hline & $k A_{c s}$ & $A_{c s}$ & $V_{\tau}$ \\
\hline I1 & 42.6 & 301 & 0.919 \\
\hline I2 & 22.1 & 1410 & 0.387 \\
\hline
\end{tabular}


In the next case, a model with multiple injectors and producers is tested. The test case is the homogeneous reservoir described in Section 3.3.1. It has 4 producers and 5 injectors and an isotropic permeability of $40 \mathrm{md}$. Two sets of conductivity values are obtained using two different assumptions: no injector-injector interaction and with injector-injector interaction. The conductivity values and well allocation factors for the model in which injector-injector interaction is neglected is shown in Table 3.1 and Table 3.4. Table 4.1 and Table 4.4 give the conductivity and well allocation factors in a model that considers injector-injector interaction. In addition to injector-injector conductivity, the influence of injectors on non-adjacent producers is a fact that differentiates the two models. Values of $V_{\tau}$ and a normalized values for $A_{c s}$ for the two assumptions are obtained and given in Table 6.6 and Table 6.7 for no injector-injector interaction and in Table 6.8 and Table 6.9 for injector-injector interaction. The normalized values for $A_{c s}$ are obtained by dividing the $A_{c s}$ values from the optimization routine by the average thickness of the bond. This is done to allow a more sensitive view of the $A_{c s}$ differences.

Table 6.6: Time-of-flight Dykstra-Parsons coefficients for the homogenous model from 3.3.1

\begin{tabular}{ccccc}
\hline & P1 & P2 & P3 & P4 \\
\hline I1 & 0.421 & 0.393 & 0.287 & 0.156 \\
\hline I2 & 0.546 & 0.317 & 0.376 & 0.364 \\
\hline I3 & 0.238 & 0.222 & 0.249 & 0.398 \\
\hline I4 & 0.352 & 0.290 & 0.245 & 0.373 \\
\hline I5 & 0.233 & 0.325 & 0.309 & 0.343 \\
\hline
\end{tabular}

Both assumptions give symmetric values and reflect the isotropy of the system and symmetry of well placement, although each one tells a different story when well conductivity values and well allocation factors are compared. Based on obtained well allocation factors for the no injectorinjector interaction assumption, $15 \%$ of injection goes to each non-adjacent producer. The value of normalized $A_{c s}$ is very small for non-adjacent connections, which means that a relatively high amount of flow occurs through a narrow path and should result in an early breakthrough. But in 
Table 6.7: Normalized cross-section area open to flow for the homogenous model from 3.3.1 $\left(f t^{2} / f t\right)$

\begin{tabular}{ccccc}
\hline & P1 & P2 & P3 & P4 \\
\hline I1 & 779 & 700 & 211 & 160 \\
\hline I2 & 709 & 100 & 816 & 207 \\
\hline I3 & 255 & 533 & 144 & 274 \\
\hline I4 & 90 & 639 & 115 & 780 \\
\hline I5 & 44 & 43 & 537 & 564 \\
\hline
\end{tabular}

Table 6.8: Time-of-flight Dykstra-Parsons coefficients for the homogenous model from 4.2.1

\begin{tabular}{cccccccccc}
\hline & P1 & P2 & P3 & P4 & I1 & I2 & I3 & I4 & I5 \\
\hline I1 & 0.294 & 0.488 & 0.249 & 0.077 & 0.000 & 0.000 & 0.159 & 0.000 & 0.000 \\
\hline I2 & 0.373 & 0.257 & 0.463 & 0.169 & 0.000 & 0.000 & 0.170 & 0.000 & 0.000 \\
\hline I3 & 0.283 & 0.193 & 0.250 & 0.222 & 0.159 & 0.170 & 0.000 & 0.144 & 0.132 \\
\hline I4 & 0.175 & 0.407 & 0.151 & 0.296 & 0.000 & 0.000 & 0.144 & 0.000 & 0.000 \\
\hline I5 & 0.163 & 0.205 & 0.415 & 0.350 & 0.000 & 0.000 & 0.132 & 0.000 & 0.000 \\
\hline
\end{tabular}

Table 6.9: Normalized cross-section area open to flow for the homogenous model from 4.2.1 $\left(f t^{2} / f t\right)$

\begin{tabular}{cccccccccc}
\hline & P1 & P2 & P3 & P4 & I1 & I2 & I3 & I4 & I5 \\
\hline I1 & 830 & 771 & 1023 & 446 & 0 & 0 & 923 & 0 & 0 \\
\hline I2 & 590 & 335 & 807 & 263 & 0 & 0 & 780 & 0 & 0 \\
\hline I3 & 2120 & 295 & 616 & 727 & 923 & 780 & 0 & 849 & 446 \\
\hline I4 & 525 & 1143 & 168 & 970 & 0 & 0 & 849 & 0 & 0 \\
\hline I5 & 680 & 603 & 709 & 677 & 0 & 0 & 446 & 0 & 0 \\
\hline
\end{tabular}

reality, water injection in adjacent wells causes breakthrough in producers. Well allocation factors for the model that considers injector-injector interaction is zero. This means that matching each phase production is not sensitive to values of $V_{\tau}$ and $A_{c s}$, and these parameters do not affect the model. Therefore, one can say the well allocation factor determines the sensitivity of the model to 
the two-phase flow model parameters. Because this is an inverse problem, parameters that do not influence the model can take any values, but their values are of minor importance.

In addition to non-adjacent well connections, the model that considers injector-injector interaction results in $40 \%$ more reduction in the difference between observed and modeled individual phase production. In other words, it matched observed production better. Therefore, the comparison shows the importance of including injector-injector interaction.

The next candidate to try the two-phase network model on is a heterogeneous system with $Y$ directional permeability trend. This case is discussed in section 4.2.2. Conductance values and WAFs that are shown in Figure $4.5 a$ and $b$ depict the strong influence from injectors 1 and 4 on producer 2 and from injectors 2 and 4 on producer 3 . Injector 3 also supports producer 1 and 4 more than the other two producers. All the mentioned connections are on vertical lines in the map view of the system. This reflects the $Y$-direction permeability trend.

Values of $V_{\tau}$ and Normalized $A_{c s}$ are given in Table 6.10 and Table 6.11. The highest value of $V_{\tau}$ for each injector is obtained for a producer in the vertical direction which is the same direction that is mentioned above. This is a good indication of heterogeneity in the $Y$ direction.

The case with a high permeability channel was first introduced in section 3.3.6. Conductance values and WAFs for the model that considers injector-injector interaction were obtained in section 4.2.3 and are shown in Figure $4.12 a$ and $b$. Values of $V_{\tau}$ and Normalized $A_{c s}$ are given in Table 6.12 and Table 6.13. All injectors except injector 5 demonstrate higher $V_{\tau}$ with producer 2 , which means they are connected to producer 2 through a heterogeneous feature. In addition, the fact the Normalized $A_{c s}$ value between injector 2 and producer 2 is very small means that flow between this well-pair occurs through a narrow area, which agrees with the existence of high-permeability channel between them.

\subsection{Conclusion}

In order to solve the two-phase network model, two-phase flow parameters like phase conductivity need to be known. The goal here is to obtain the saturation distribution to determine phase con- 
Table 6.10: Time-of-flight Dykstra-Parsons coefficients for $Y$-direction permeability trend from 4.2.2

\begin{tabular}{cccccccccc}
\hline & P1 & P2 & P3 & P4 & I1 & I2 & I3 & I4 & I5 \\
\hline I1 & 0.196 & 0.569 & 0.429 & 0.167 & 0.000 & 0.000 & 0.211 & 0.000 & 0.000 \\
\hline I2 & 0.303 & 0.139 & 0.519 & 0.057 & 0.000 & 0.000 & 0.161 & 0.000 & 0.000 \\
\hline I3 & 0.299 & 0.016 & 0.125 & 0.570 & 0.211 & 0.161 & 0.000 & 0.298 & 0.305 \\
\hline I4 & 0.165 & 0.381 & 0.117 & 0.020 & 0.000 & 0.000 & 0.298 & 0.000 & 0.000 \\
\hline I5 & 0.441 & 0.380 & 0.545 & 0.272 & 0.000 & 0.000 & 0.305 & 0.000 & 0.000 \\
\hline
\end{tabular}

Table 6.11: Normalized cross-section area open to flow for $Y$-direction permeability trend from 4.2.2 $\left(f t^{2} / f t\right)$

\begin{tabular}{cccccccccc}
\hline & P1 & P2 & P3 & P4 & I1 & I2 & I3 & I4 & I5 \\
\hline I1 & 730 & 581 & 649 & 196 & 0 & 0 & 144 & 0 & 0 \\
\hline I2 & 622 & 348 & 743 & 537 & 0 & 0 & 172 & 0 & 0 \\
\hline I3 & 295 & 860 & 179 & 660 & 144 & 172 & 0 & 185 & 774 \\
\hline I4 & 414 & 1037 & 346 & 1575 & 0 & 0 & 185 & 0 & 0 \\
\hline I5 & 1719 & 591 & 393 & 684 & 0 & 0 & 774 & 0 & 0 \\
\hline
\end{tabular}

ductivity at any distance from the injectors. The saturation distribution is specifically needed at node interfaces (bonds) and producers. It is important at the interface, since it defines the phase conductivity of the bond to the fluids that flow from the injector node to the producer node. It is also important at the producer since, it controls the fractional flow of fluids at the producer. To obtain the fluid distribution between injector-producer pairs, saturation is mapped on hypothetical streamlines between the injector and producer. The average time of flight of the center streamline and the time-of-flight Dykstra-Parson coefficient characterize a set of streamlines between the injector-producer pair. The average time of flight is the mean value of time of flight between the pair. It is a function of the total flow rate between the pair, distance between the pair, and the cross section area open to flow $\left(A_{c s}\right)$. All the parameters that define average time of flight are known except $A_{c s}$, which is a match parameter. Now one may ask, why is the average time of flight not a 
Table 6.12: Time-of-flight Dykstra-Parsons coefficients for high permeability channel from 4.2.3

\begin{tabular}{cccccccccc}
\hline & P1 & P2 & P3 & P4 & I1 & I2 & I3 & I4 & I5 \\
\hline I1 & 0.218 & 0.500 & 0.207 & 0.142 & 0.000 & 0.000 & 0.243 & 0.000 & 0.000 \\
\hline I2 & 0.240 & 0.444 & 0.271 & 0.225 & 0.000 & 0.000 & 0.325 & 0.000 & 0.000 \\
\hline I3 & 0.052 & 0.275 & 0.167 & 0.269 & 0.243 & 0.325 & 0.000 & 0.144 & 0.153 \\
\hline I4 & 0.212 & 0.570 & 0.206 & 0.232 & 0.000 & 0.000 & 0.144 & 0.000 & 0.000 \\
\hline I5 & 0.212 & 0.102 & 0.104 & 0.278 & 0.000 & 0.000 & 0.153 & 0.000 & 0.000 \\
\hline
\end{tabular}

Table 6.13: Cross-section area open to flow for high permeability channel from $4.2 .3\left(f t^{2} / f t\right)$

\begin{tabular}{cccccccccc}
\hline & P1 & P2 & P3 & P4 & I1 & I2 & I3 & I4 & I5 \\
\hline I1 & 163 & 860 & 1819 & 214 & 0 & 0 & 264 & 0 & 0 \\
\hline I2 & 599 & 191 & 245 & 136 & 0 & 0 & 365 & 0 & 0 \\
\hline I3 & 290 & 825 & 43 & 123 & 264 & 365 & 0 & 167 & 130 \\
\hline I4 & 308 & 493 & 553 & 291 & 0 & 0 & 167 & 0 & 0 \\
\hline I5 & 108 & 215 & 241 & 206 & 0 & 0 & 130 & 0 & 0 \\
\hline
\end{tabular}

match parameter instead of the $A_{c s}$, since the average time of flight is the parameter that characterizes the streamline? The answer is, in addition to the time of flight from injector to the producer, the time of flight of the points between the well pairs should be determined. So a bond-shape domain is needed to obtain the velocity profile and the time of flight at each point between a pair. This shape will help capture the characteristics of the fractional flow behavior better than simply some average. Heterogeneity between a pair is incorporated into the system through the time-of-flight Dykstra-Parson coefficient. The $V_{\tau}$ value varies between 0 and 1 , and a value closer to 1 means a more heterogeneous system.

As was discussed earlier, fractional flow models have difficulty matching the water production values from the field. Using fractional flow models correspond to using one streamline between each well-pair. That is why in this model, a set of streamlines leaves the injector to mimic the field behavior. But there is a tradeoff between the number of streamlines between a well-pair and computational time. In Figure 6.18, an attempt to find an optimum number of streamlines is illus- 


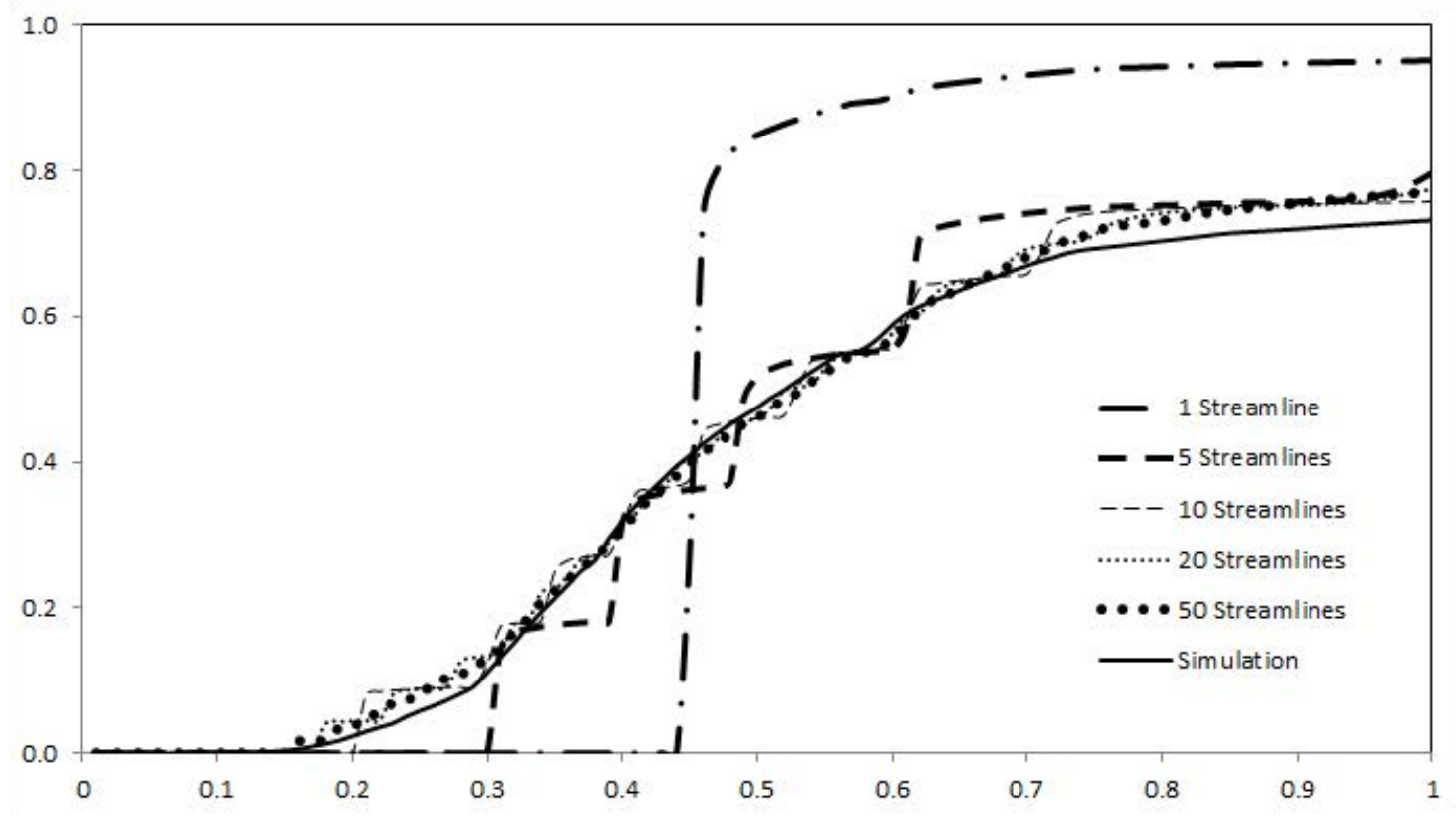

Figure 6.18: The optimum number of streamlines between injector-producer pairs is the smallest number of streamlines that matches simulation curve.

trated. In this figure, the fractional flow at the producer is plotted versus dimensionless time for the homogeneous case discussed in section 6.10. Each line represents a case in which a different number of streamlines connect the injector-producer pair. One can notice that whenever the front in one of the streamlines reaches the producer, the fractional flow curve jumps. For example, in the case that there are five streamlines between an injector-producer pair, there are five jumps in the fractional flow curve. After increasing the number of streamlines to 20, it would be difficult to see the jumps, and a smooth curve is obtained. It also matched the simulation fractional flow curve.

One of the limitations of this method is obtaining unrealistic saturation values in injector nodes. Table 6.14 shows the node saturation at the end of the study period. Although a simple calculation shows that the average saturation matches the model saturation, individual node saturations are not real and sometimes don't have physical meaning. There are two reasons for this behavior. First of all, the focus has been on matching the fractional flow at producers, and not enough attention has been paid to other aspects of the model, especially properties of the injector nodes. Secondly, the diamond shape that is used to obtain the velocity profile may not be representative of the 
Table 6.14: Obtained Saturation in five blocks in the model

\begin{tabular}{cccccc}
\hline & Producer 1 & Producer 2 & Producer 3 & Producer 4 & Injector 1 \\
\hline Saturation & 0.33 & 0.34 & 0.34 & 0.34 & 1.93 \\
\hline
\end{tabular}

velocity behavior of the streamlines connecting the injector and the producer. The diamond results in a linear velocity profile (Figure 6.4a), but in practice velocity profiles are tortuous and may be characterized better by a parabolic velocity profile.

To test this hypothesis, an ellipse-shaped tube was used to obtain the velocity profile (Figure 6.19). Cross-sectional area at any point between an injector-producer pair is obtained from:

$$
y= \pm \sqrt{\frac{a^{2} b^{2}-a^{2} x^{2}}{a^{2}}}
$$

where $2 a$ is the distance between well-pairs, $2 b$ is the cross-sectional area open to flow, and $2 y$

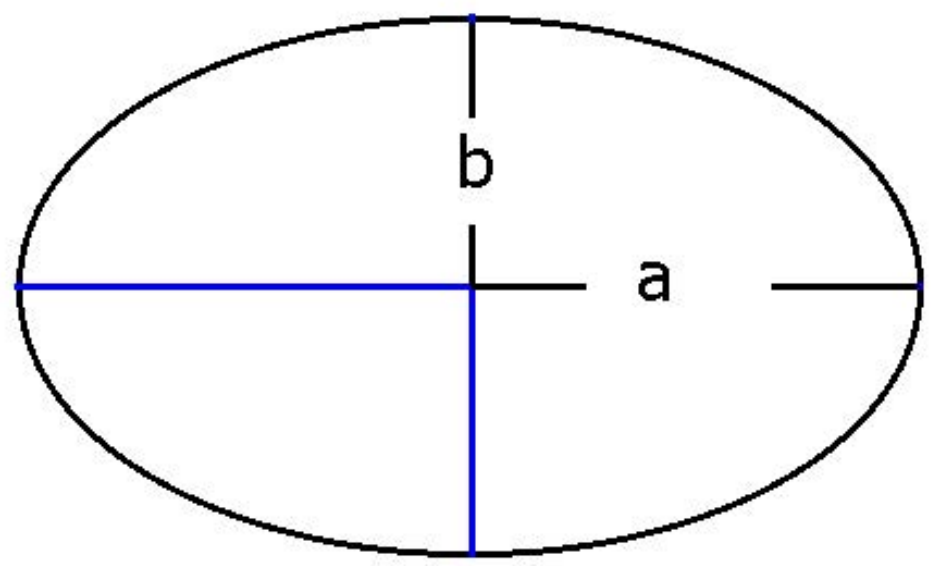

Figure 6.19: Ellipse-shape tube.

is the cross-sectional area at distance $x$ from the injector. This gives a parabolic-shaped velocity profile. The velocity changes very rapidly around the wells and very slowly away from the wells. This gives a longer average time of flight compared to the diamond shape streamtube for a specific area open to flow. As a result, the optimization program gives a smaller area open to flow for an ellipse-shaped tube, although the saturation values are the same. Therefore, the ellipse-shaped tube doesn't help in obtaining realistic saturation distributions. 
A second hypothesis is that the wrong node volumes were used. Node volume sizes are a function of distance between wells, and with equal distances between wells, equal size node volume will be calculated. But by looking at the cases where saturation values larger than 1 were obtained, one finds that cumulative rate plays an important role. For example, for the $1 D$ case with 1 injector and 1 producer, the computed saturation distribution is $70 \%$ for the injector and $46 \%$ for the producer. This makes sense, and its average equals $58 \%$, which is equal to the model average saturation obtained from the finite difference simulator used to generate the case. But in the case with 1 injector and 4 producers, injection rate is four times larger than each production rate, although the same node volume sizes are assigned to all injectors. In the case with 4 injectors and 5 producers, cumulative injection rates are larger in injector 1 and injector 2 compared to other injectors, and those are the wells that obtained saturation values larger that 1.

By assuming that the $L$ term in the conductance formula is equal to the shortest distance between wells, one could extract $k A_{c s}$ from conductance. This chapter proposed a method to obtain $A_{c s}$. Now that all elements of conductance except $k$ are available, the question is, would it be possible to backcalculate the permeability used in the original simulation model used to generate the data for the homogeneous case. Dividing average $k A_{c s}$ between adjacent injector-producer pairs in Table 4.2 by average $A_{c s}$ between adjacent injector-producer pairs in Table 6.9 gives a permeability of 22 $m d$, which is less than the $40 \mathrm{md}$ permeability of the homogeneous system. This underestimation is the consequence of the assumptions that was made to obtain $L$ and $A_{c s}$. $L$ is assumed to be the shortest distance between an injector-producer pair; however, the actual path that a particle travels to reach the producer is longer than the shortest distance between the well-pair (in the finite difference simulator, fluid flows from grid cell to grid cell). Therefore, the actual $L$ is longer than the shortest distance between an injector-producer well-pair. In addition, the cross-sectional area open to flow is assumed to be the largest section of the diamond shape streamtube between an injector-producer pair, although average cross-sectional area open to flow should be narrower than the assumed value. For a value of conductance, a longer $L$ and a narrower $A_{c s}$ both result in a larger 
$k$. Therefore, the expectation would be that obtaining the $40 \mathrm{md}$ permeability would be achieved if the actual values of $L$ and $A_{c s}$ were known and used.

In this chapter a tool was developed that provides a degree of heterogeneity between the injectorproducer pairs. In addition, it provides the cross-sectional area open to flow, which gives insight into the area swept between well-pairs. But most importantly, it computes the fractional flow of water that flows from the injector node to the producer node and to the production well. Injection may be reallocated to decrease the high water fraction flow and increase low water fractional flow. This shows the ability of the method to be a waterflood optimization tool in addition to be a reservoir characterization tool. 


\section{Chapter 7}

\section{Summary, conclusions and future work}

\subsection{Summary}

In this work, a two-step approach to find the conductance between injector-producer pairs and characterize heterogeneity between well-pairs is proposed. The first step is a single-phase model that gives conductance between injector- producer pairs similar to existing approaches. The second step is a two-phase model that determines time-of-flight Dykstra-Parson coefficients and crosssectional areas open to flow between well-pairs.

To obtain conductance values, injection and production rates and well positions are required. To find the time-of-flight Dykstra-Parsons coefficients and cross-sectional areas open to flow, fractional flow at the producers is needed in addition to well rates and well positions. The conductance values obtained from the first step are also required to run the two-phase model.

Interaction between injectors was modeled by considering a bond between injectors. This modification resulted in an increase in adjacent injector-producer influence and a decrease in influence of non-adjacent wells. In addition, obtained conductance values and well allocation factors represent the domain better than a model in which injector-injector connections are ignored.

The method was applied on a portion of a waterflood field case with 22 injectors and 27 producers. Bottomhole pressure information was available for most of the producers in the system. The method was adapted for the case of an open boundary, in which there is flow into and out of the system. In addition to converting a producer to an injector, changes in the productivity of the producers and part-time injection and production were modeled.

Fractional flow was matched at the producers in the two-phase network model to obtain timeof-flight Dykstra-Parsons coefficients and cross-sectional areas open to flow for each bond in the system. The method was applied to many synthetic fields, with different reservoir features like 
directional permeability and high permeability cannel to see if obtained parameters reflect the reservoir features.

\subsection{Conclusions}

The following conclusions stem from this dissertation:

1. The method is fast and can be easily set up. It resolves some of the limitations that the currently available methods have. It calculates conductance values, which have a physical meaning that still must be interpreted. This approach considers changes in flow pattern due to shuting-in a producer for a long time, adding or removing wells or changes in well rate. This allows the method to study a broader time span. It also allows testing a wider range of injection-production scenarios in the prediction phase.

2. The model needs a rough estimate of bottomhole pressures and well indices. Incorrect values of bottomhole pressure and well indices still gives correct values of well allocation factors, but errors in BHP and/or well indicies will be reflected in compensating errors in local values of conductance. Accurate values of conductance are needed when one tries to extract actual reservoir parameters from the conductance. When the focus is on improving the flood efficiency the errors in the numerical value of the conductance values are of minor importance and well allocation factors become important.

3. Errors in estimating reservoir volume and fluid compressibility will be more widespread than errors in bottomhole pressure and well indices and influence the entire set of conductance values and well allocation factors. Since percent error in estimating reservoir boundary, reservoir thickness, porosity, and displacing and displaced fluid compressibility is typically not very large, errors in these values should not have a great influence on the result.

4. In spite of errors and uncertainties in the input parameters of the network model, which is a part of every reservoir study project and previous connectivity models, results are useful and 
can be relied on. What differentiates a reliable network model is whether it leads to better decisions. All of the equally probable solutions that are obtained by using different sets of uncertain parameters, in spite of minor difference in degree of influence between well pairs, show the same connections and lead to the consistent decisions.

5. By considering interaction between adjacent injectors, the influence of injectors on nonadjacent producers was explained. The obtained parameters differentiate between the indirect influence a non-adjacent injector may have on a producer through another injector and the direct influence the same injector may have on a producer through a direct flow path. Both cases were interpreted simply as a connection between non-adjacent injector-producer pairs before including injector-injector interactions.

6. Although the approach can be applied to water injection cases, the results may not be reasonable for all of injection cases. One of the requirements to obtain a reliable solution is that data have noticeable pulses in injectors and producers. Production rate changes as a result of these pulses should be larger than errors in the production data.

7. Two-phase flow model results show that including injector-injector interactions provide more reasonable values of the time-of-flight Dykstra-Parsons coefficients and cross-sectional areas open to flow than when these interactions are not included. In the network model, channels are characterized by large time-of-flight Dykstra-Parsons coefficients and narrow crosssectional areas open to flow. The obtained parameters are good indications of the degree of sweep between injector-producer pairs.

\subsection{Future work}

In the proposed large-scale network model, it was assumed that conductance of bonds represent reservoir properties between injector-producer well pairs. The first goal was to infer physical properties like permeability from conductance. But there are a number of concerns in extracting permeability from conductance. 
First is the uncertainty associated with the values of the other parameters in the conductance term. If one traces streamlines, streamline geometries can change due to injection and production rate fluctuations (Thiele 2001). The number of streamlines, the area covered by them, and the paths they travel define streamline geometry, all of which depend on the pressure gradient and permeability field. In chapter 6 , the cross-sectional area covered by streamlines and the average length of the streamlines were related to the area open to flow and length in the network model for an injector-producer pair. But area open to flow and length for bonds in the network model is a function of rate changes, and the shortest distance between well pairs may not be a good representation of the actual path that fluids travel. Thus the role of $A_{c s}$ and $L$ should be studied before trying to evaluate a particular value of $k$ for each bond.

A second concern is the applicability of relating the calculated parameters to something "real". Values of conductance between specific injectors and associated producers reflect the relative distance and permeability between them. For another injection well in the same homogeneous reservoir with the same specification, we should expect the same values of conductance. But when applying the network model to a few large test cases, values differ significantly from one injector to another when large well indices are assumed for the injectors. Therefore, estimated values of conductance should be improved in order to infer properties from them if it appears that assumed well indices play a significant role.

This approach is based on the idea that injection fluctuations change production rates in producers surrounding the injector. The more noticeable the changes are, the easier to find the degree of influence of injector on each producer. This influence may be large enough to cover the error in the data. For example, if changes in production rates as a result of injection fluctuations are larger than errors generated by production rate measurements, using the network model approach is reasonable. Another example is a case in which BHP data are not available. If changes in production rates due to injection fluctuations are greater than the change in production as BHP changes, then one can still use the method, even when BHP data are not available. On the other hand, if injection rates are almost constant and other factors like changes in BHP are sources of production rate changes, 
then using the method would not be recommended. Therefore, before trying to apply the network model to field data, an evaluation of the data and reservoir and fluid properties like permeability and compressibility should be performed to see if using the method is feasible.

At each step during the course of obtaining conductance values one has the ability to check the difference between observed and modeled production rates. It is recommended that the problem set up be checked to see if the difference between observed and modeled production is very large. The initial guess is important since it has a great impact on the difference. When the difference is very large, then it wouldn't be sensitive to the variation in the conductance values, and the optimization may not converge, or it may converge to a local minimum. Therefore, it is better to check the difference between observed and modeled production rates for each well to find if there is discrepancy in the input data.

In addition to reservoir properties used in the single-phase network model, one needs an estimation of irreducible water and residual oil saturation in the two-phase network model. If the method is being applied on mature waterfloods, some initial estimate of water saturation at the time being modeled is also needed. Like BHP and WI, a good estimation of irreducible water and residual oil saturation leads to obtaining more physically viable results. One can also use the approach applied by Sayarpour et al. (2011). They used several equally probable sets of uncertain reservoir parameters to match a production history with CRM. They then developed the consequent cumulative distribution functions for the uncertain variables. These cumulative distribution functions were compared to that of finite difference simulation to evaluate the reliability of the method.

Chapter 6 explained that two saturation equations are being solved in two-phase network model. One is a pseudo- $1 D$ equation along the streamlines that finds the relative conductivity values, and the second is a saturation equation that updates the node volume saturation. Obtaining nodevolume saturation is of minor importance, and it doesn't give any information about reservoir properties. One can still match oil and water production and obtain interwell properties without calculating node-volumes saturation. This is possible just by using single-phase flow equations and pseudo- $1 D$ equation along the streamlines. In other words, the set up may change in a way 
that the second saturation equation that updates node-volume saturation would not be required. This modification may provide the opportunity to match water and oil production for one producer at a time instead of considering all producers collectively. This helps to study certain regions of interest without the requirement to evaluate the entire study area, and as result could increase the speed and performance of the method. To increase the performance, one can also group adjacent injectors to decrease the number of injector-producer connections and as result the number of variables. Grouping the producers is not recommended, since they have different productivity and BHP, and each one should be treated separately.

The next step after calibrating the network model parameters with the historical production/injection data is to predict future performance and optimize oil production. These are the ultimate goals of any waterflood performance study technique. The optimization function could maximize the amount of oil produced or one can step further and include economic variables to maximize net present value (NPV). Reallocating water injection is the first optimization plan that should be considered. If one can control production rate, then adjusting BHP or total production rate would be another choice. 


\section{Bibliography}

Ahmed, Tarek. 2010. Reservoir Engineering Handbook. Burlington, MA: Gulf Professional Publishing.

Albertoni, Alejandro. 2002. "Inferring Interwell Connectivity From Well-Rate Fluctuations in Waterfloods." Master's thesis, The U. of Texas at Austin.

Albertoni, Alejandro, and Larry W. Lake. 2003. "Inferring Interwell Connectivity Only From Well-Rate Fluctuations in Waterfloods." SPE Reservoir Evaluation and Engineering 6 (1): 6-16 (February).

Arps, J.J. 1945. "Analysis of Decline Curves." Transactions of the AIME 160 (1): 228-247 (December).

Bakke, Stig, and Pal E. Øren. 1997. “3-D Pore-Scale Modelling of Sandstones and Flow Simulations in the Pore Network." SPE Journal 2 (14): 136-149 (January 23). SPE-35479-PA.

Bansal, Y., and M. Sayarpour. 2012, 8-10 October. "Fault-Block Transmissibility Estimation Using Injection and Production Data in Waterfloods." SPE Annual Technical Conference and Exhibition. San Antonio, Texas. SPE-159067-MS.

Batycky, Roderick P. 1997. "A Two-Dimensional Two-phase Field Scale Streamline Simulator." Ph.D. diss., Stanford University.

Can, B., and C. S. Kabir. 2012, 8-10 October. "Simple Tools for Forecasting Waterflood Performance." SPE Annual Technical Conference and Exhibition. San Antonio, Texas. SPE156956-MS.

Chaudhry, Amanat U. 2004. Oil Well Testing Handbook. Burlington, MA: Society of Petroleum Engineers.

Christie, M.A., and M.J. Blunt. 2001. "Tenth SPE Comparative Solution Project: A Comparison of Upscaling Techniques." SPE Reservoir Evaluation and Engineering 4 (4): 308-317 (August).

Deutsch, Clayton, and Andre Journal. 1998. GSLIB Geostatistical Software Library and User's Guide. Oxford.

Dinh, A. V., and D. Tiab. 2008, September 21-24. "Interpretation of Interwell Connectivity Tests in a Waterflood System." SPE Annual Technical Conference and Exhibition. Denver, Colorado. SPE-116144-MS.

Dykstra, H., and R. L. Parsons. 1950. The Prediction of Oil Recovery by Waterflooding. Secondary Recovery of Oil in the United States.

Ershaghi, Iraj, and Doddy Abdassah. 1984. "A Prediction Technique for Immiscible Processes Using Field Performance Data.” Journal of Petroleum Technology 36 (4): 664-670 (April).

Ertekin, Turgay, J.H. Abou-Kassem, and G.R. King. 2001. Basic Applied Reservoir Simulation. Richardson, Texas: Elsevier.

Farrashkhalvat, M, and J Miles. 2003. Basic Structured Grid Generation with an introduction to unstructured grid generation. Oxford: Butterworth Heinemann. 
Izgec, O., and C.S. Kabir. 2009, 24-26 March. "Establishing Injector-Producer Connectivity Before Breakthrough During Fluid Injection.” SPE Western Regional Meeting. Jose, California. SPE-121203-MS.

Izgec, O., and Shah Kabir. 2010. "Quantifying Nonuniform Aquifer Strength at Individual Wells." SPE Reservoir Evaluation and Engineering 13 (2): 296-305 (April).

Kabir, C.S., and B. Izgec. 2009, 4-7 October. "Identification and Characterization of HighConductive Layers in Waterfloods." SPE Annual Technical Conference and Exhibition. New Orleans, Louisiana. SPE-123930-MS.

Kaviani, D., P. Valko, and J.L. Jensen. 2010, April 24-28. "Application of the Multiwell Productivity Index-Based Method to Evaluate Interwell Connectivity." SPE Improved Oil Recovery Symposium. Tulsa, Oklahoma. SPE-129965-MS.

Kaviani, Danial, Jerry L. Jensen, Larry W. Lake, and Mashhad Fahes. 2008, November 3-6. "Estimation of Interwell Connectivity in the Case of Fluctuating Bottomhole Pressures." Abu Dhabi International Petroleum Exhibition and Conference. Abu Dhabi, UAE. SPE-117856MS.

Lee, John. 1982. Well Testing. Society of Petroleum Engineers.

Lee, Kun-Han, Antonio Ortega, Amir Mohammad Nejad, and Iraj Ershaghi. 2008, March 29April 2. "A Method for Characterization of Flow Units Between Injection-Production Wells Using Performance Data." SPE Western Regional and Pacific Section AAPG Joint Meeting. Bakersfield, California. SPE-114222-MS.

Lee, Kun-Han, Antonio Ortega, Amir Mohammad Nejad, Nelia Jafroodi, and Iraj Ershaghi. 2009, March 24-26. "A Novel Method for Mapping Fractures and High Permeability Channels in Waterfloods Using Injection and Production Rates." SPE Western Regional Meeting. San Jose, California. SPE-121353-MS.

Liang, X., Hunan Changsha, D.B. Weber, T.F. Edgar, L.W. Lake, M. Sayarpour, and A. Al-Yousef. 2007, April 1-3. "Optimization of Oil Production Based on a Capacitance Model of Production and Injection Rates.” Hydrocarbon Economics and Evaluation Symposium. Dallas, Texas. SPE-107713-MS.

Malik, Mohammad A. 1988, 9-11 November. "A Shock-Free Approach in Fractional Flow Theory.” SPE Eastern Regional Meeting. Pittsburgh, Pennsylvania. SPE-153912-MS.

Mattax, Calvin C., and Robert L. Dalton. 1990. Reservoir Simulation. Richardson, TX: Society of Petroleum Engineers.

Oliver, Dean S., Albert C. Reynolds, and Ning Liu. 2008. Inverse Theory for Petroleum Reservoir Characterization and History Matching. Cambridge: Cambridge University Press.

Panda, M.N., and A.K. Chopra. 1998, February 17-19. "An Integrated Approach to Estimate Well Interactions." SPE India Oil and Gas Conference and Exhibition. New Delhi, India. SPE-39563-MS.

Pande, K. K., H. J. Ramey Jr., W. E. Brigham, and F. M. Orr Jr. 1987, 8-10 April. "Frontal Advance Theory for Flow in Heterogeneous Porous Media." SPE California Regional Meeting. Ventura, California. SPE-16344-MS. 
Parekh, B., and C. S. Kabir. 2011, 30 October-2 November. "Improved Understanding of Reservoir Connectivity in an Evolving Waterflood with Surveillance Data." SPE Annual Technical Conference and Exhibition. Denver, Colorado. SPE-146637-MS.

Refunjol, B.T., and Larry W. Lake. 1997, March 2-4. "Reservoir characterization based on tracer response and rank analysis of production and injection rates." Forth International Reservoir Characteristics Technical Conference. Houston, Texas.

Samier, P., L. Quettier, and M. Thiele. 2001, February 11-14. "Applications of Streamline Simulations to Reservoir Studies.” SPE Reservoir Simulation Symposium. Houston, Texas. SPE66362-MS.

Satter, Abdus, Ghulam M. Iqbal, and James L. Buchwalter. 2007. Practical Enhanced Reservoir Engineering. Tulsa, Oklahoma: PennWell Corp.

Sayarpour, M., , C.S. Kabir, and L.W. Lake. 2009. "Field Applications of Capacitance-Resistance Models in Waterfloods." SPE Reservoir Evaluation and Engineering 12 (6): 853-864 (December).

Sayarpour, M., C.S. Kabir, K. Sepehrnoori, and Larry W. Lake. 2011. "Probabilistic history matching with the capacitanceresistance model in waterfloods: A precursor to numerical modeling." Journal of Petroleum Science and Engineering 78 (1): 96-108 (July).

Sayarpour, M., E. Zuluaga, C.S. Kabir, and Larry W. Lake. 2007, 11-14 November. "The Use of Capacitance-Resistive Models for Rapid Estimation of Waterflood Performance." SPE Annual Technical Conference and Exhibition. Anaheim, California. SPE-110081-MS.

Sayarpour, Morteza. 2008. "Development and Application of Capacitance-Resistive Models to Water/CO2 Flood." Ph.D. diss., The U. of Texas at Austin.

Shahvali, M., S.G. Shirzadi, P.R. Ballin, and E. Ziegel. 2012, 21-23 March. "Waterflood Management Based on Well Allocation Factors for Improved Sweep Efficiency: Model Based or Data Based?" SPE Western Regional Meeting. Bakersfield, California. SPE-153912-MS.

Thiele, Marco R. 2001, September 3-7. "Streamline Simulation." 6th International Forum on Reservoir Simulation. Schloss Fuschl, Austria.

Thiele, Marco R., and Rod P. Batycky. 2006. "Using Streamline-Derived Injection Efficiencies for Improved Waterflood Management." SPE Reservoir Evaluation and Engineering 9 (2): 187-196 (April). SPE-84080-PA.

Thiele, M.R., R.P. Batycky, M.J. Blunt, and F.M. Orr. 1996. "Simulating Flow in Heterogeneous Systems Using Streamtubes and Streamlines." SPE Reservoir Engineering 11 (1): 5-12 (February). SPE-27834-PA.

Wang, Wenli, Tad W. Patzek, and Larry W. Lake. 2011, 30 October-2 November. "A CapacitanceResistive Model and InSAR Imagery of Surface Subsidence Explain Performance of a Waterflood Project at Lost Hills." SPE Annual Technical Conference and Exhibition. Denver, Colorado. SPE-146366-MS.

Welge, Henry J. 1952. “A Simplified Method for Computing Oil Recovery by Gas or Water Drive." Journal of Petroleum Technology 4 (4): 91-98 (April).

Yang, Zhengming. 2009. “A New Diagnostic Analysis Method for Waterflood Performance." SPE Reservoir Evaluation and Engineering 12 (9): 341-351 (April). 
Yousef, A.A., L.W. Lake, and J.L. Jensen. 2009, 22-26 April. "Analysis and Interpretation of Interwell Connectivity From Production and Injection Rate Fluctuations Using a Capacitance Model." SPE-DOE Symposium on Improved Oil Recovery. Tulsa, Oklahoma. SPE-99998MS.

Yousef, Ali A., Pablo Gentil, Jerry L. Jensen, and Larry W. Lake. 2006. "A Capacitance Model To Infer Interwell Connectivity From Production- and Injection-Rate Fluctuations." SPE Reservoir Evaluation and Engineering 9 (9): 630-646 (December). SPE-95322-PA.

Zemel, Bernard. 1995. Tracers in the Oil Field. Amsterdam, The Netherlands: Elsevier.

Zhang, Hongchao, Andrew R. Conn, and Katya Scheinberg. 2010. "A Derivative-Free Algorithm for Least-Aquares Minimization.” SIAM J. OPTIM. 20 (6): 3555-3576 (December). 


\section{Appendix A: Nomenclature}

\section{Roman Symbols}

$\begin{array}{ll}A_{c S I J} & \text { area open to flow between two connected nodes } I \text { and } J\left(f t^{2}\right) \\ B & \text { formation volume factor }(R B / S T B) \\ c & \text { comressibility }(1 / p s i) \\ C_{D} & N_{m} \times N_{m} \text { covariance matrix for the data measurement } \\ D & \text { diffusion coefficient } \\ f_{p} & \text { water fractional flow } \\ g_{I J} & \text { conductance between two connected nodes } I \text { and } J(d . f t / c p) \\ J_{w} & \text { productivity index }(S T B / \text { day } / p s i) \\ k_{I J} & \text { permeability between two connected nodes } I \text { and } J(d) \\ k_{r} & \text { relative permeability } \\ L_{I J} & \text { distance between two connected nodes } I \text { and } J(f t) \\ N_{D} & \text { number of observations } \\ N_{m} & \text { number of variables } \\ p & \text { pressure }(p s i) \\ q_{I J} & \text { volumetric flow rate between two connected nodes } I \text { and } J(b b l) \\ q_{m o d} & \text { assumed theoretical model for predicting data measurement }(S T B) \\ q_{o b s} & N_{D} \text { dimensional column vector containing measured data }(S T B) \\ R_{p} & \text { ratio of time on production to the data collection interval time } \\ S & \text { saturation } \\ u & \text { velocity }(f t / s e c) \\ V_{b} & \text { bulk volume of the node }\left(f t^{3}\right) \\ V_{\tau} & \text { time-of-flight Dykstra-Parsons coefficient } \\ V_{s l} & \text { volume of the bond }\left(f t^{3}\right) \\ Z & \text { coordination number }\end{array}$

\section{Greek Symbols}

$\alpha_{c} \quad$ volumetric conversion factor (5.615)

$\beta_{c} \quad$ transmissibility conversion factor (1.127)

$\mu_{I J} \quad$ viscosity between two connected nodes $I$ and $J(c p)$

$\phi \quad$ porosity

$\sigma \quad$ standard deviation

$\tau \quad$ time of flight (days) 


\section{Indices and Special Subscripts}

$\begin{array}{ll}I & =\text { node volume containing injector } i \\ J & =\text { node volume containing producer } j \\ i & =\text { injector index } \\ j & =\text { producer index } \\ o & =\text { oil } \\ w & =\text { water }\end{array}$




\section{Appendix B: Underdetermined and Overdetermined Problems}

Although a reservoir produces continuously, discrete inverse problems are considered to represent it. In a discrete problem, a physical system like a reservoir is defined by a finite number of model variables.

The $N_{m}$ model variables are represented by a vector $m$

$$
m=\left[m_{1}, m_{2}, \ldots, m_{M}\right]^{T}
$$

The $N_{d}$ calculated data values can be represented by a vector $d$

$$
d=\left[d_{1}, d_{2}, \ldots, d_{N_{d}}\right]^{T}
$$

If we study a linear system, a discerte inverse problem can be represented by

$$
d=G m
$$

where $G$ is an $N_{d} \times N_{m}$ matrix representing the sensitivity of the data to the model variables. Observed data can be represented by a vector $d_{o b s}$. If the measured data are exact (zero measurement error) and $m$ is the true (actual) physical model, then $d$ will be identical to measured data (Oliver et al. 2008) which means:

$$
d_{o b s}=G m
$$

It can also be shown that Eq.B.4 has a unique solution for every $d_{o b s}$ in the data space if all of the rows of $G$ are independent and the number of model variables $N_{m}$ is equal to the number of data $N_{d}$ (Oliver et al. 2008). On the other hand, if $N_{m}>N_{d}$ then the problem is underdetermined.

For the case where the number of independent rows or columns (the rank of the $N_{d} \times N_{m}$ matrix $G$ ) is less than $N_{d}$, there exist vectors $d_{o b s}$ in the data space which are not in the range of $G$. Such problems are overdetermined. 


\section{Appendix C: Flowcharts}

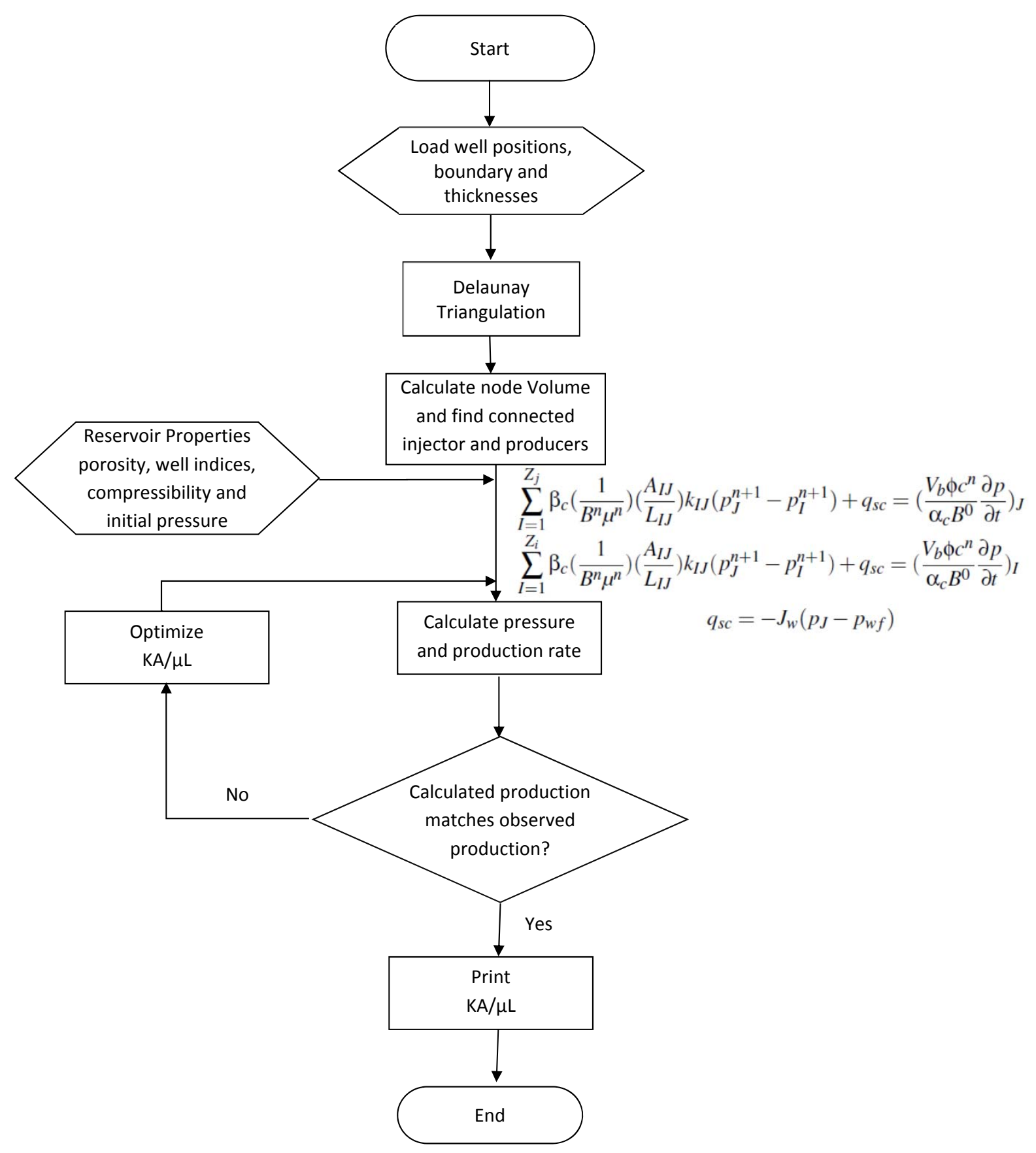

Figure C.1: Flowchart showing how single phase network model works. 


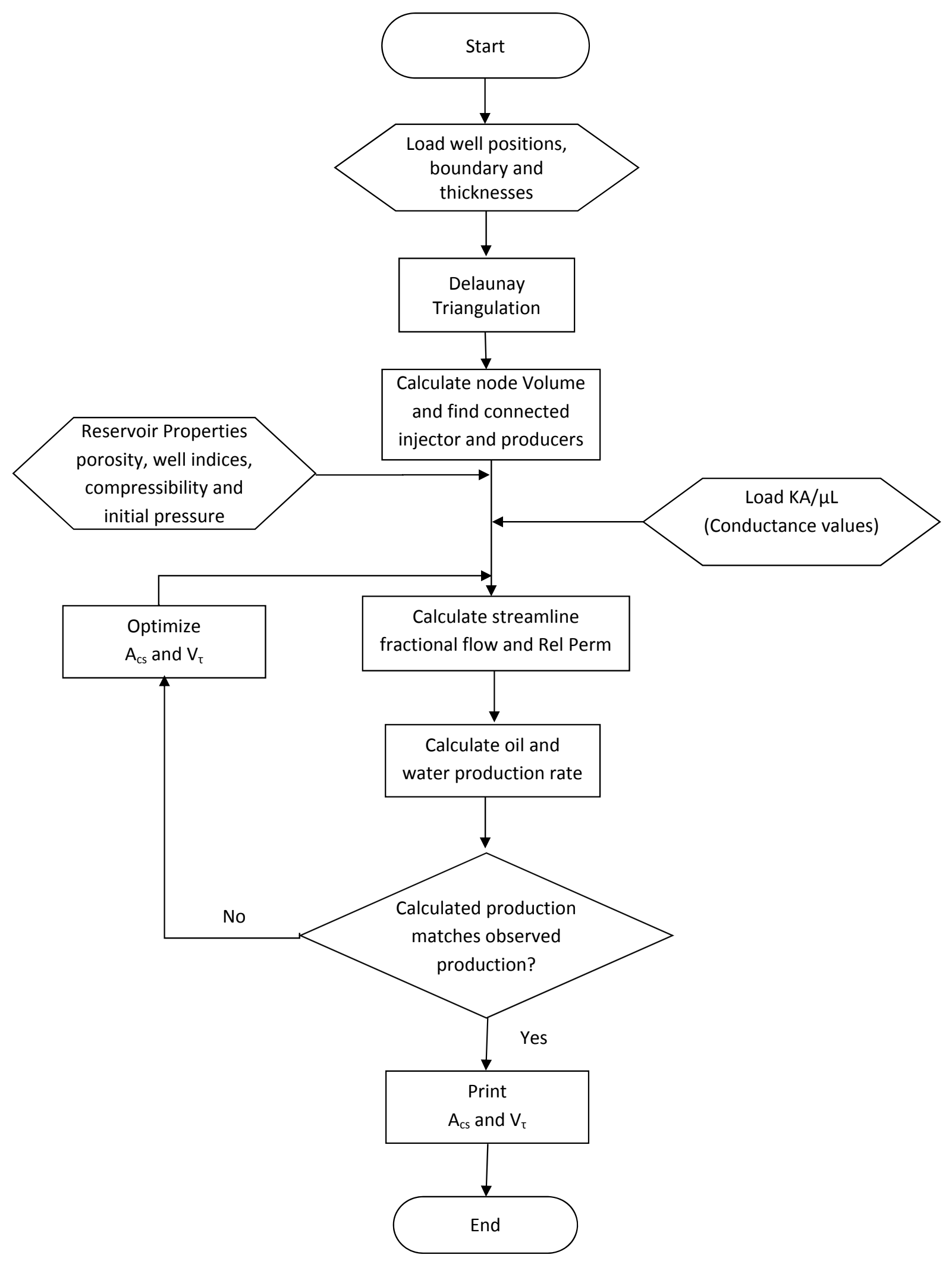

Figure C.2: Flowchart showing how two phase network model works. 


\section{The Vita}

SeyedMohammadAmin Gherabati was born in Abadan, Iran. He finished his undergraduate studies at Petroleum University of Technology, Abadan, in May 2005. He earned a master of science degree in chemical engineering from Amirkabir University of Technology in August 2007. He is currently a candidate for the degree of Doctor of Philosophy in petroleum engineering, to be awarded in December 2013. 